\title{
Computational Studies on the Mechanism and Origin of the Different Regioselectivities of Manganese Porphyrin-Catalyzed C-H Bond Hydroxylation and Amidation of Equilenin Acetate
}

Liyuan Jin, Qunmin Wang, Xiahe Chen, Ning Liu, Xiaoli Fang, Yun-Fang Yang*, and Yuan-Bin She*

College of Chemical Engineering, Zhejiang University of Technology, Hangzhou, Zhejiang 310014, China

\author{
*E-mail: yangyf@zjut.edu.cn \\ *E-mail: sheyb@zjut.edu.cn
}

\section{Table of Contents}

Figure S1. (a) Optimized transition state structures of TS2b and TS4. (b) Optimized

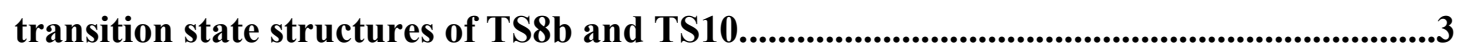

Figure S2. IRC pathway for the ${ }^{3}$ TS4, ${ }^{5}$ TS4, ${ }^{3}$ TS10 and ${ }^{5}$ TS10 ...................................4

Figure S3. Optimized geometries of diradical $\sigma$-complex INT4a and zwitterionic

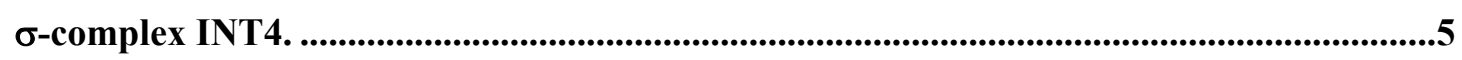

Figure S4. Populations of NPA charges $(Q$ in e) and spin densities ( $\rho)$ for the transition state structures involved in HAT for $\mathrm{C}\left(s p^{3}\right)-\mathrm{H}$ bond hydroxylation and OAT for $\mathrm{C}\left(s p^{2}\right)-\mathrm{H}$ bond hydroxylation.

Figure S5. Hammett plots of $\log \left(k_{\mathrm{R}} / k_{\mathrm{H}}\right)$ vs. $\sigma_{\mathrm{p}}^{+}$for the hydroxylation of para-substituted toluene. $\left(\mathrm{R}={ }^{t} \mathrm{Bu}, \mathrm{Me}, \mathrm{F}, \mathrm{H}, \mathrm{COOH}\right)$

Figure S6. Optimized transition state structures of ${ }^{3} \mathrm{TS} 4-\mathrm{m} 1,{ }^{3} \mathrm{TS} 2 \mathrm{~b}-\mathrm{m} 1,{ }^{3} \mathrm{TS} 4-\mathrm{m} 2$, and ${ }^{3}$ TS2b-m2.

Figure S7. Resonance structures of hydroxylation reaction at C-6 and C-7 reaction site.9 
Figure S8. Populations of NPA charges ( $Q$ in e) and spin densities ( $\rho)$ for NAT transition state TS10 and the $\sigma$-complex intermediate INT9 in $\mathrm{C}\left(s p^{2}\right)-\mathrm{H}$ amidation...........................9

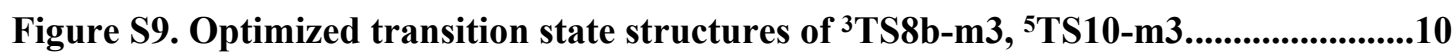
Table S1. The Calculated Free Energies of ${ }^{3}$ TS2b and ${ }^{3}$ TS4 using Different Functionals

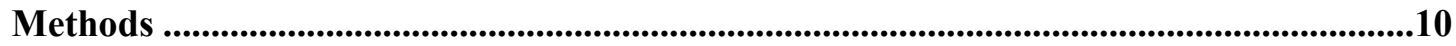

Table S2. Electronic Energies, Enthalpies, and Free Energies (in Hartree) of the Structures. ..........................................................................................................................................10

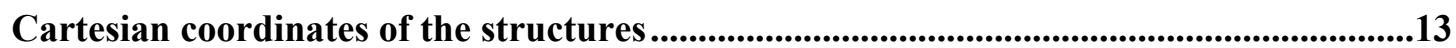


a)

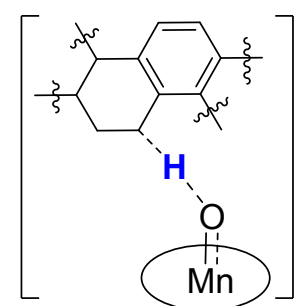

${ }^{1} \mathrm{TS} 2 \mathrm{~b} \Delta \Delta G_{\text {gas }}^{\ddagger}=11.3 \mathrm{kcal} / \mathrm{mol}$

${ }^{3} \mathrm{TS} 2 \mathrm{~b} \quad \Delta \Delta G_{\text {gas }}^{\ddagger}=0.5 \mathrm{kcal} / \mathrm{mol}$

${ }^{5} \mathrm{TS} 2 \mathrm{~b} \quad \Delta \Delta G_{\text {gas }}^{\ddagger}=3.9 \mathrm{kcal} / \mathrm{mol}$

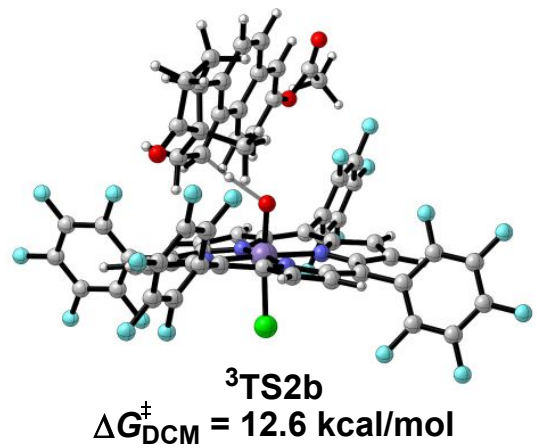

b)

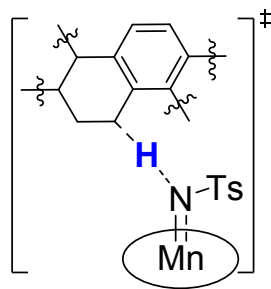

${ }^{1} \mathrm{TS} 8 \mathrm{~b} \quad \Delta \Delta G_{\mathrm{gas}}^{\ddagger}=9.2 \mathrm{kcal} / \mathrm{mol}$

${ }^{3} \mathrm{TS} 8 \mathrm{~b} \quad \Delta \Delta G_{\text {gas }}^{\ddagger}=0.0 \mathrm{kcal} / \mathrm{mol}$

${ }^{5} \mathrm{TS} 8 \mathrm{~b} \quad \Delta \Delta G_{\text {gas }}^{\ddagger}=2.1 \mathrm{kcal} / \mathrm{mol}$

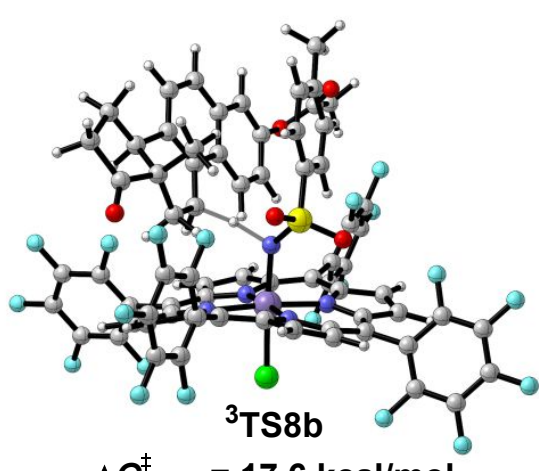

$\Delta G_{\mathrm{DCM}}^{\ddagger}=17.6 \mathrm{kcal} / \mathrm{mol}$

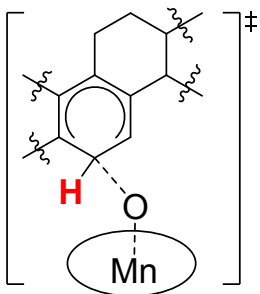

${ }^{1} \mathrm{TS} 4 \quad \Delta \Delta G_{\mathrm{gas}}^{\ddagger}=12.3 \mathrm{kcal} / \mathrm{mol}$

${ }^{3} \mathrm{TS} 4 \quad \Delta \Delta G_{\text {gas }}^{\ddagger}=0.0 \mathrm{kcal} / \mathrm{mol}$

${ }^{5} \mathrm{TS} 4 \quad \Delta \Delta G_{\text {gas }}^{\ddagger}=5.1 \mathrm{kcal} / \mathrm{mol}$

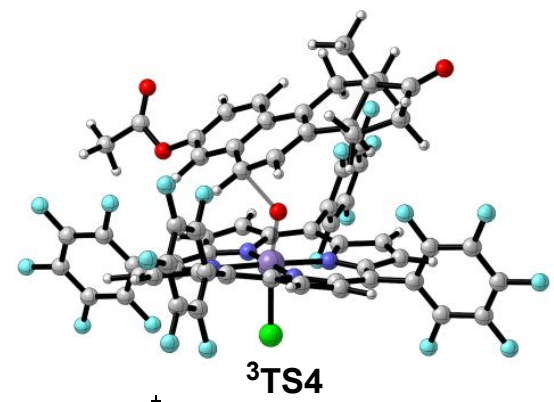

$\Delta G_{\mathrm{DCM}}^{\ddagger}=11.0 \mathrm{kcal} / \mathrm{mol}$

$$
\begin{aligned}
& { }^{1} \mathrm{TS} 10 \quad \Delta \Delta G_{\text {gas }}^{\ddagger}=12.1 \mathrm{kcal} / \mathrm{mol} \\
& { }^{3} \mathrm{TS} 10 \quad \Delta \Delta G_{\text {gas }}^{\ddagger}=3.2 \mathrm{kcal} / \mathrm{mol} \\
& { }^{5} \mathrm{TS} 10 \quad \Delta \Delta G_{\text {gas }}^{\ddagger}=4.3 \mathrm{kcal} / \mathrm{mol}
\end{aligned}
$$

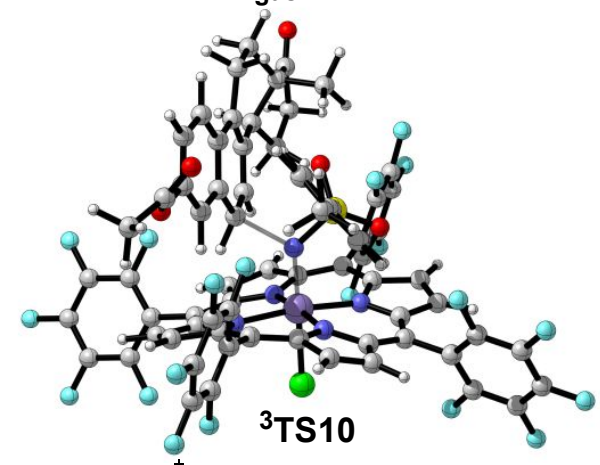

$\Delta G_{\mathrm{DCM}}^{ \pm}=22.7 \mathrm{kcal} / \mathrm{mol}$

Figure S1. (a) Optimized transition state structures of TS2b and TS4. Gibbs free energies in gas-phase were obtained at the UB3LYP-D3/6-31G(d)-LANL2DZ level of theory. (b) Optimized transition state structures of TS8b and TS10. Gibbs free energies in gas-phase were obtained at the UB3LYP-D3/6-31G(d)-LANL2DZ level of theory. 

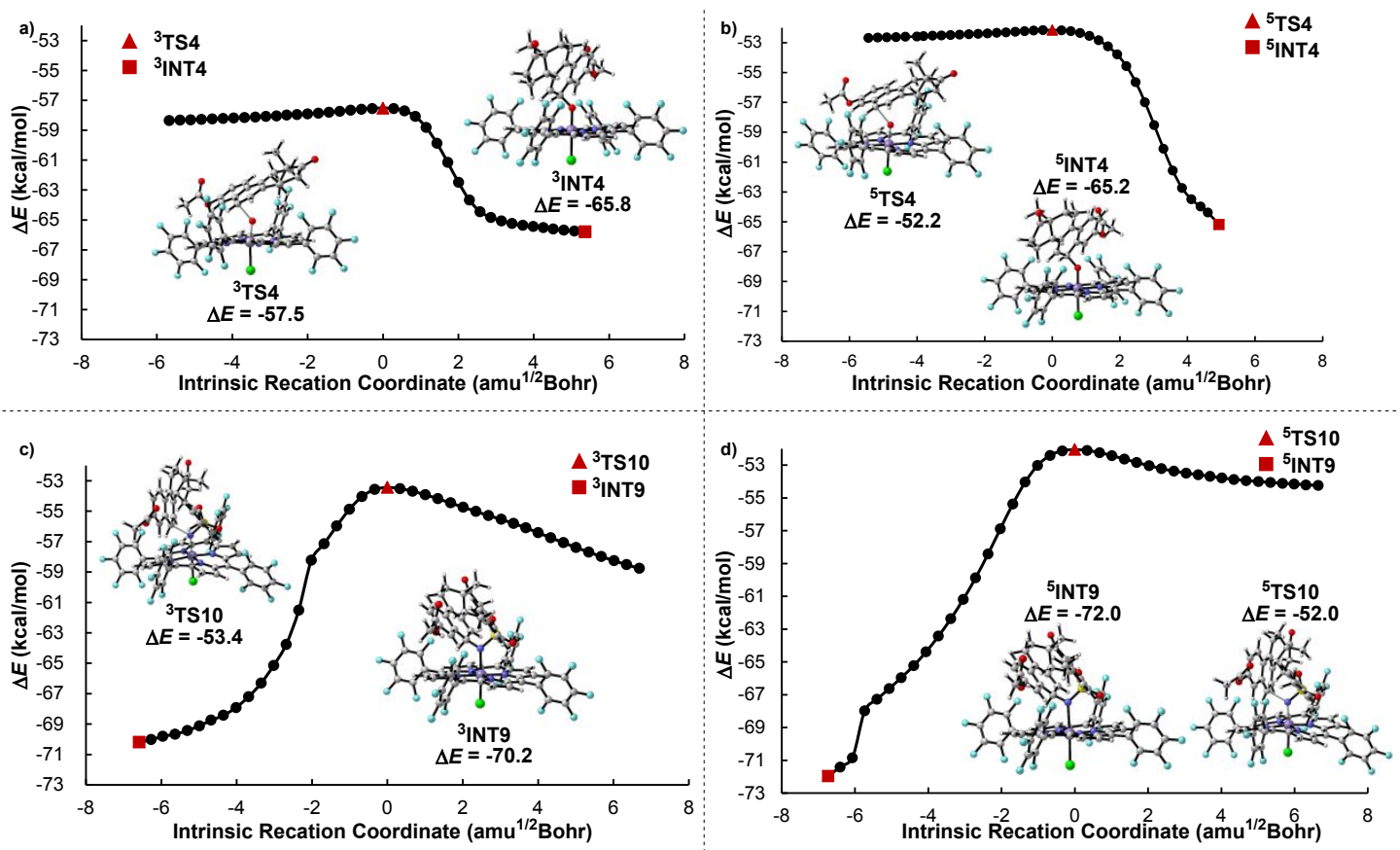

Figure S2. IRC pathway for the ${ }^{3}$ TS4, ${ }^{5}$ TS4, ${ }^{3}$ TS10 and ${ }^{5}$ TS10.

Figure S3 shows the optimized geometries of INT4 and INT4a. In diradical $\sigma$-complex ${ }^{3} \mathbf{I N T 4 a}$, the $\mathrm{C}-\mathrm{O}$ and $\mathrm{C}-\mathrm{H}$ distances are $1.44 \AA$ and $1.10 \AA$, respectively. The corresponding distances in zwitterionic $\sigma$-complex ${ }^{3} \mathbf{I N T} 4$ are $1.14 \AA$ for $\mathrm{C}-\mathrm{O}$ and $1.16 \AA$ for $\mathrm{C}-\mathrm{H}$, respectively. The zwitterionic $\sigma$-complex ${ }^{3}$ INT4 is more stable than the diradical $\sigma$-complex ${ }^{3} \mathbf{I N T}$ 4a by $4.7 \mathrm{kcal} / \mathrm{mol}$. The $\mathrm{C}-\mathrm{O}$ bond is shortened upon formation of the zwitterionic $\sigma$-complex ${ }^{\mathbf{3}} \mathbf{I N T}$. The $\mathrm{C}-\mathrm{O}$ and $\mathrm{C}-\mathrm{H}$ distances of the quintet state ${ }^{\mathbf{5}} \mathbf{I N T} \mathbf{4} /{ }^{\mathbf{5}} \mathbf{I N T} \mathbf{4 a}$ are similar with that of their triplet state structures. 

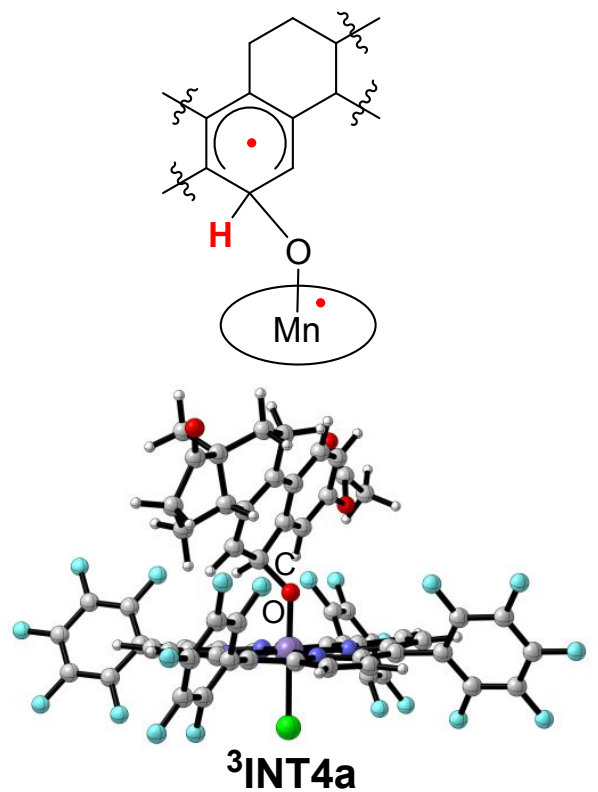

$0.1 \mathrm{kcal} / \mathrm{mol}$

C-H: 1.10

C-O: 1.44

Mn-O: 1.79

$\angle \mathrm{Mn}-\mathrm{O}-\mathrm{C}: 131.4$

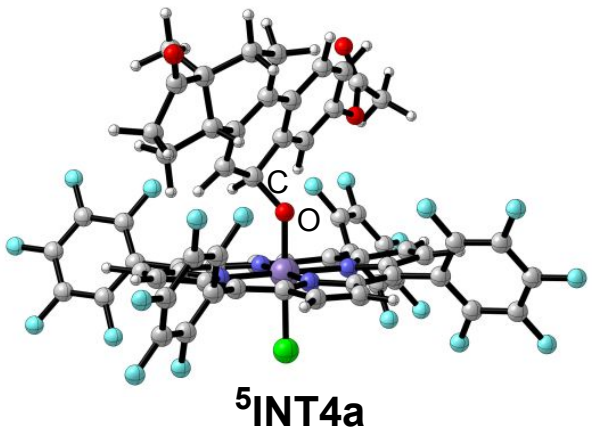

$0.5 \mathrm{kcal} / \mathrm{mol}$

C-H: 1.10

C-O: 1.45

Mn-O: 1.80

LMn-O-C: 132.6
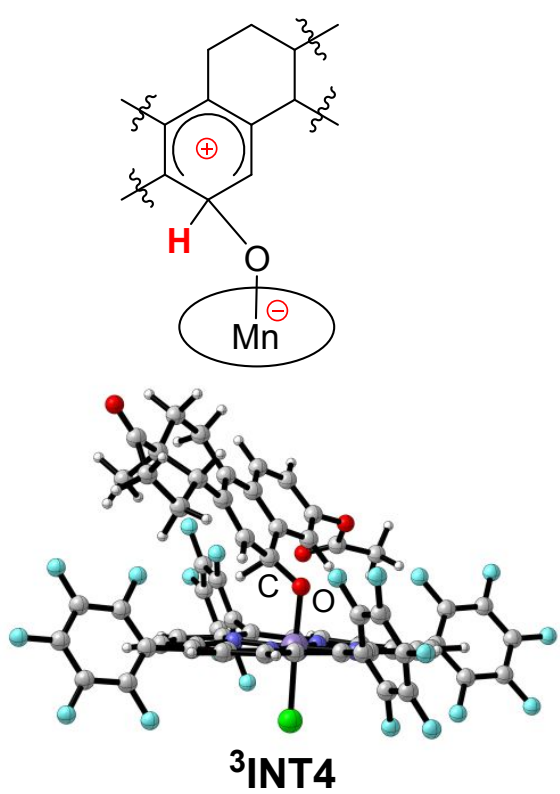

$-4.6 \mathrm{kcal} / \mathrm{mol}$

C-H: 1.16

C-O: 1.14

Mn-O: 1.89

$\angle \mathrm{Mn}-\mathrm{O}-\mathrm{C}: 124.4$

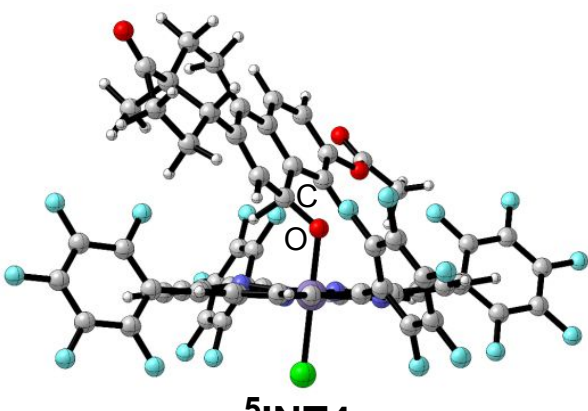

5INT4

\section{$-2.8 \mathrm{kcal} / \mathrm{mol}$}

C-H: 1.16

C-O: 1.36

Mn-O: 1.94

$\angle \mathrm{Mn}-\mathrm{O}-\mathrm{C}: 123.1$

Figure S3. Optimized geometries of diradical $\sigma$-complex INT4a and zwitterionic $\sigma$-complex INT4. The distances are in $\AA$. The angles are in degrees. 


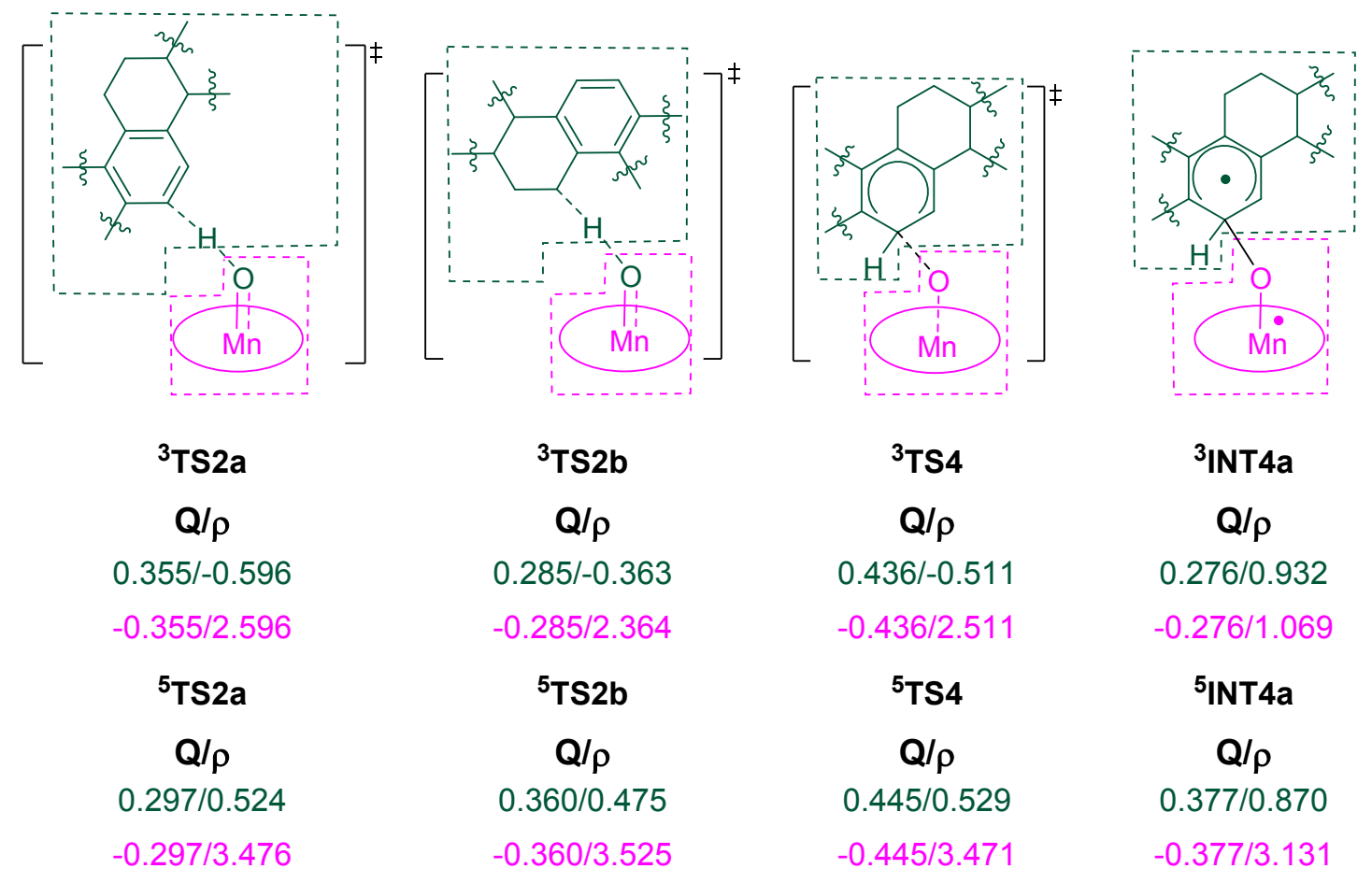

Figure S4. Populations of NPA charges (Q in e) and spin densities $(\rho)$ for the transition state structures involved in HAT for $\mathrm{C}\left(s p^{3}\right)-\mathrm{H}$ bond hydroxylation and OAT for $\mathrm{C}\left(s p^{2}\right)-\mathrm{H}$ bond hydroxylation.

The HAA transition states for benzylic $\mathrm{C}\left(s p^{3}\right)-\mathrm{H}$ bond hydroxylation of para-substituted toluene were also calculated. As shown in Figure S5, most of the $\log \left(k_{\mathrm{R}} / k_{\mathrm{H}}\right)$ varies from -0.1 to 0.1 , which are not sensitive to the para-substituent. The small Hammett $\rho$ values, approximately -0.32 , indicates that there is little substituent effect in the HAA process that has been observed in previous studies (references: a) Wang, Y.; Kumar, D.; Yang, C.; Han, K.; Shaik, S. Theoretical Study of N-Demethylation of Substituted N,N-Dimethylanilines by Cytochrome P450: The Mechanistic Significance of Kinetic Isotope Effect Profiles. J. Phys. Chem. B 2007, 111, 7700-7710. b) Boess, E.; Wolf, L. M.; Malakar, S.; Salamone, M.; Bietti, M.; Thiel, W.; Klussmann, M. Competitive Hydrogen Atom Transfer to Oxyl- and Peroxyl Radicals in the Cu-Catalyzed Oxidative Coupling of N-Aryl Tetrahydroisoquinolines Using tert-Butyl Hydroperoxide. ACS Catal. 2016, 6, 3253-3261). Therefore the Hammett analysis strongly supports that the $\mathrm{C}\left(s p^{2}\right)-\mathrm{H}$ 
bond hydroxylation undergoes the OAT mechanism while the $\mathrm{C}\left(s p^{3}\right)-\mathrm{H}$ bond hydroxylation undergoes the HAA/OR mechanism.

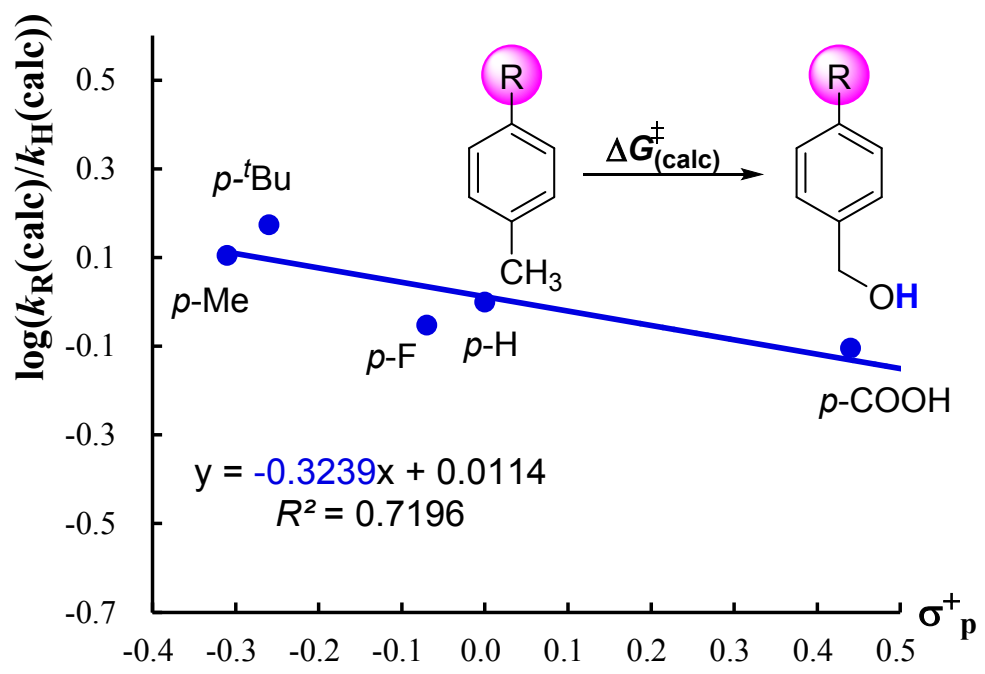

Figure S5. Hammett plots of $\log \left(k_{\mathrm{R}} / k_{\mathrm{H}}\right)$ vs. $\sigma_{\mathrm{p}}^{+}$for the hydroxylation of para-substituted toluene. $\left(\mathrm{R}={ }^{t} \mathrm{Bu}, \mathrm{Me}, \mathrm{F}, \mathrm{H}, \mathrm{COOH}\right)$

Figure S6 shows the optimized geometries and relative Gibbs free energies of key transition states of the two truncated models. In model I, toluene was used as substrate, $\mathrm{Mn}(\mathrm{TFPP}) \mathrm{Cl}$ was used as catalyst, and the $\mathrm{C}\left(s p^{2}\right)-\mathrm{H}$ bond hydroxylation is disfavored by $3.1 \mathrm{kcal} / \mathrm{mol}$ compared with benzylic $\mathrm{C}\left(s p^{3}\right)-\mathrm{H}$ bond hydroxylation. In model II, steroid equilenin acetate was used as substrate, $\mathrm{Mn}(\mathrm{THP}) \mathrm{Cl}$ (THP, porphine) was used as catalyst, and the $\mathrm{C}\left(s p^{2}\right)-\mathrm{H}$ bond hydroxylation is disfavored by 2.5 $\mathrm{kcal} / \mathrm{mol}$ compared with benzylic $\mathrm{C}\left(s p^{3}\right)-\mathrm{H}$ bond hydroxylation. So the truncated model study suggests that both the large conjugation effect of steroid equilenin acetate and the electron-withdrawing pentafluorophenyl group at meso position of manganese porphyrin are essential to achieve the electrophilic addition of Mn-oxo to the C-6 position of steroid equilenin acetate. 


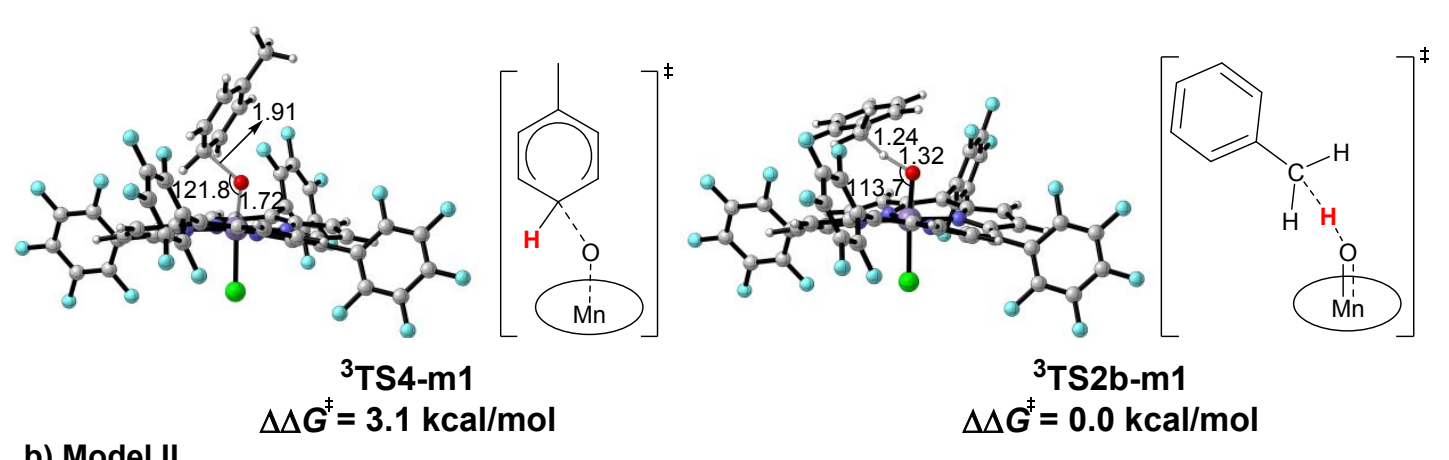

b) Model II
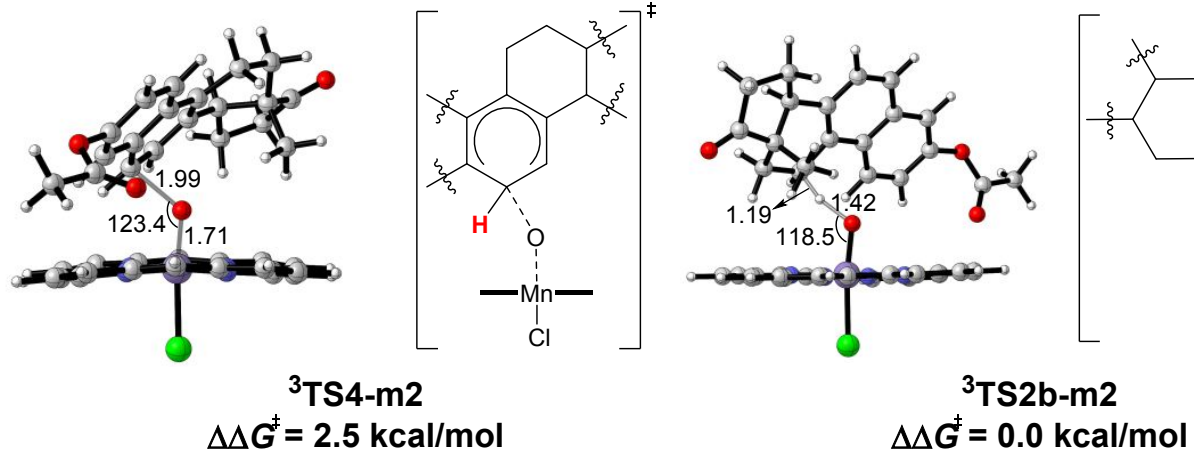

Figure S6. Optimized transition state structures of ${ }^{3}$ TS4-m1, ${ }^{3}$ TS2b-m1, ${ }^{3}$ TS4-m2, and ${ }^{3}$ TS2b-m2. The distances are in $\AA$. The angles are in degrees.

When substitution takes place at C6 of substrate, the resulting intermediate has five resonance structures, and two of them have intact benzene ring and two $p-\pi$ conjugations, as shown in red in Figure S7. When substitution takes place at C7 of substrate, the resulting intermediate also has five resonance structures, and only one of them has intact benzene ring and one $p-\pi$ conjugations, as shown in blue in Figure S7. The electrophilic aromatic substitution of substrate tends to be easier with the more extended conjugation in the Wheland intermediates. Therefore in OAT mechanism, the electrophilic aromatic substitution takes place at C6 rather than $\mathrm{C} 7$. 

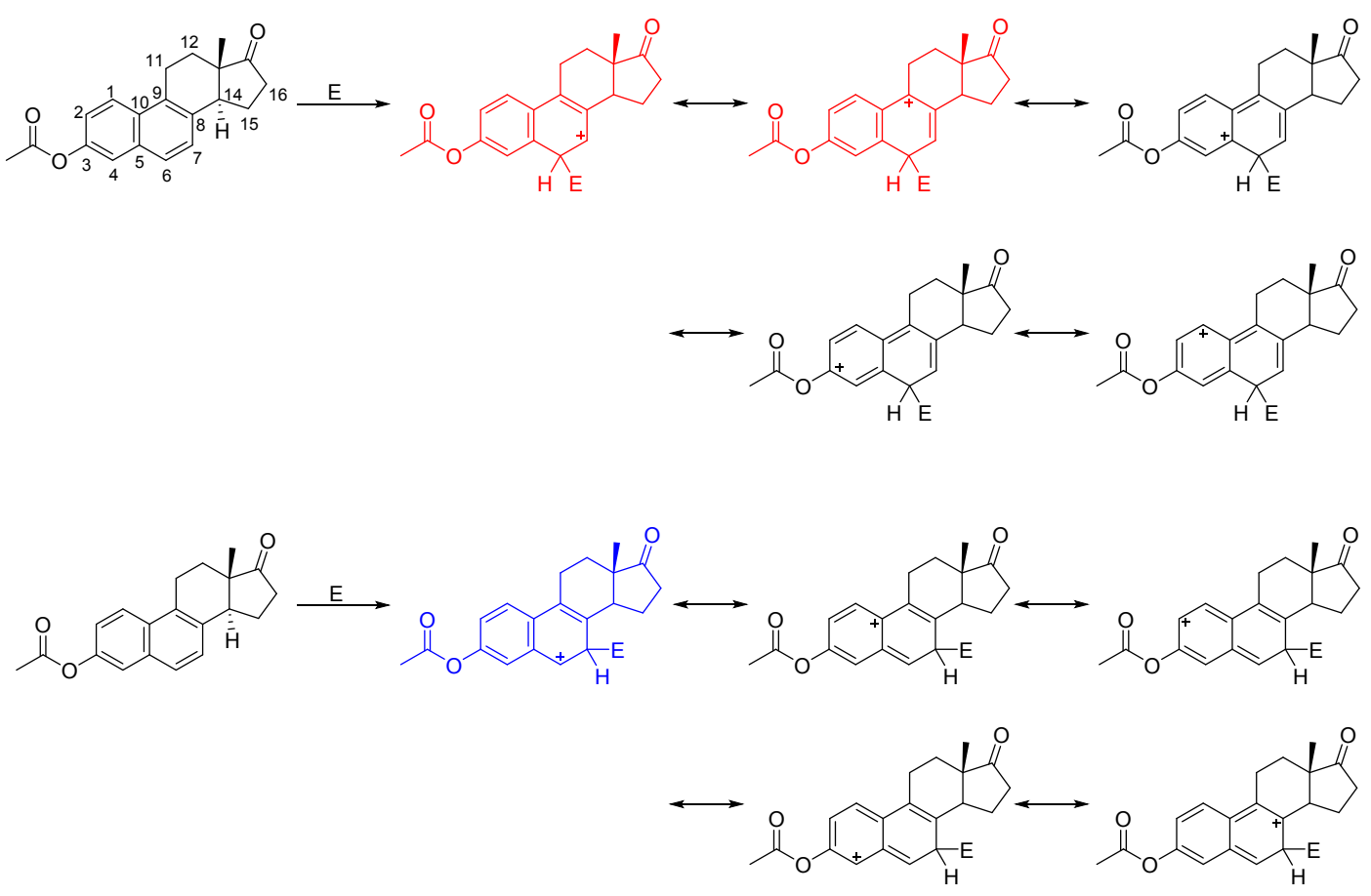

Figure S7. Resonance structures of hydroxylation reaction at C-6 and C-7 reaction site.

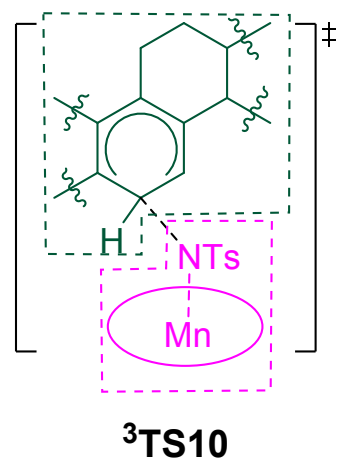

$\mathrm{Q} / \rho$

$0.433 /-0.603$

$-0.433 / 2.606$

${ }^{5} \mathrm{TS} 10$

Q/p

$0.436 / 0.624$

$-0.436 / 3.376$

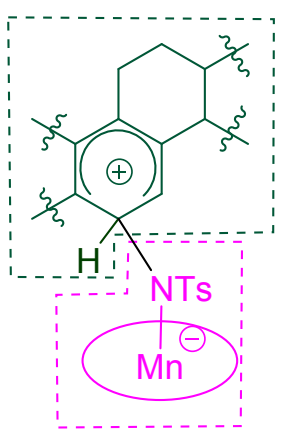

${ }^{3}$ INT9

Q/p

$0.914 / 0.003$

$-0.914 / 1.997$

${ }^{5}$ INT9

Q/ $/ \rho$

$0.831 / 0.003$

$-0.831 / 3.997$

Figure S8. Populations of NPA charges (Q in e) and spin densities ( $\rho$ ) for NAT transition state TS10 and the $\sigma$-complex intermediate INT9 in $\mathrm{C}\left(s p^{2}\right)-\mathrm{H}$ amidation. 
To understand the origin of the regioselectivity, we further computed the truncated model with $\mathrm{Mn}=\mathrm{NH}$ instead of $\mathrm{Mn}=\mathrm{NTs}$ (Figure S9). In this truncated model study, the free energy difference between $\mathrm{C}\left(s p^{3}\right)-\mathrm{H}$ bond and $\mathrm{C}\left(s p^{2}\right)-\mathrm{H}$ bond amidation transition states is $4.5 \mathrm{kcal} / \mathrm{mol}$, which is very close to the $4.9 \mathrm{kcal} / \mathrm{mol}$ difference in the full model study. So this truncated model study suggests that steric effect is small, while the electronic effect is essential to achieve the $\mathrm{C}\left(s p^{3}\right)-\mathrm{H}$ bond amidation selectivity.
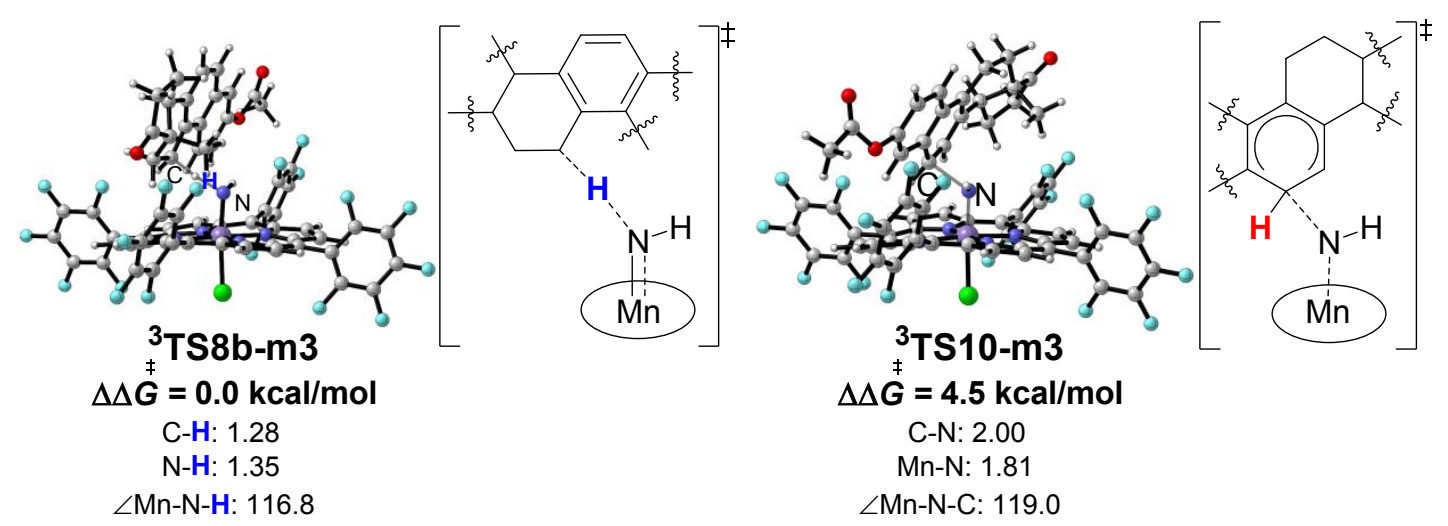

Figure S9. Optimized transition state structures of ${ }^{3}$ TS8b-m3, ${ }^{5}$ TS10-m3. The distances are in $\AA$. The angles are in degrees.

Table S1. The Calculated Free Energies of ${ }^{3}$ TS2b and ${ }^{3}$ TS4 4 using Different Functionals Methods

\begin{tabular}{|c|c|c|c|}
\hline \multirow{3}{*}{ Entry } & \multirow{3}{*}{ Computational Methods } & \multirow{3}{*}{$\begin{array}{c}{ }^{3} \text { TS2b } \\
\Delta \Delta G^{*} \\
\text { kcal/mol }\end{array}$} & \multirow{3}{*}{$\begin{array}{c}{ }^{3} \mathrm{TS4} \\
\Delta \Delta G^{*} \\
\text { kcal/mol }\end{array}$} \\
\hline & & & \\
\hline & & & \\
\hline 1 & UB3LYP-D3/def2-TZVP(SMD:Dichloromethane)//UB3LYP-D3/6-31G(d)-LANL2DZ & 1.6 & 0.0 \\
\hline 2 & UB3LYP/def2-TZVP(SMD:Dichloromethane)//UB3LYP/6-31G(d)-LANL2DZ & 0.4 & 0.0 \\
\hline 3 & 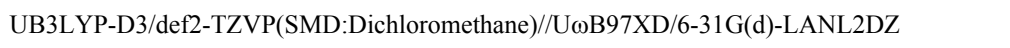 & 0.8 & 0.0 \\
\hline 4 & $\begin{array}{l}\text { UB3LYP-D3/6-311+G(d,p)-SDD(SMD:Dichloromethane)//UB3LYP-D3/6-31G(d)-LANL2D } \\
\text { Z }\end{array}$ & 2.9 & 0.0 \\
\hline 5 & UB3LYP-D3/def2-TZVP(PCM:Dichloromethane)//UB3LYP-D3/6-31G(d)-LANL2DZ & 1.4 & 0.0 \\
\hline 6 & UB3LYP-D3/6-311+G(d,p)-SDD(PCM:Dichloromethane)//UB3LYP-D3/6-31G(d)-LANL2DZ & 2.8 & 0.0 \\
\hline 7 & UM06/def2-TZVP(SMD:Dichloromethane)//UB3LYP-D3/6-31G(d)-LANL2DZ & 0.3 & 0.0 \\
\hline 8 & UM06/6-311+G(d,p)-SDD (SMD:Dichloromethane)//UB3LYP-D3/6-31G(d)-LANL2DZ & 2.2 & 0.0 \\
\hline 9 & UM06/def2-TZVP(PCM:Dichloromethane)//UB3LYP-D3/6-31G*-LANL2DZ & 0.0 & 0.0 \\
\hline 10 & UM06/6-311+G(d,p)-SDD (PCM:Dichloromethane)//UB3LYP-D3/6-31G(d)-LANL2DZ & 1.9 & 0.0 \\
\hline
\end{tabular}


Table S2. Electronic Energies, Enthalpies, and Free Energies (in Hartree) of the Structures $\begin{array}{llll}\text { Calculated at the B3LYP-D3/def2-TZVP } & \text { (SMD: }\end{array}$

Dichloromethane)//B3LYP-D3/6-31G(d)-LANL2DZ Level of Theory

\begin{tabular}{|c|c|c|c|c|c|c|c|}
\hline Structures & ZPVE & TCE & TCH & TCG & $E_{\text {sol }}$ & $G_{\text {sol }}(\mathrm{E}+\mathrm{TCG})$ & $\begin{array}{c}\text { Imaginary } \\
\text { Frequency } \\
\left(\mathrm{cm}^{-1}\right)\end{array}$ \\
\hline 1 & 0.357208 & 0.376867 & 0.377811 & 0.308649 & -1000.323757 & -1000.015108 & - \\
\hline 12 & 0.43657 & 0.49378 & 0.494724 & 0.340296 & -5510.244202 & -5509.903906 & - \\
\hline 32 & 0.436199 & 0.493519 & 0.494463 & 0.337856 & -5510.258988 & -5509.921132 & - \\
\hline 52 & 0.436033 & 0.493512 & 0.494457 & 0.337444 & -5510.28734 & -5509.949896 & - \\
\hline 3 & 0.361363 & 0.382142 & 0.383087 & 0.311687 & -1075.574674 & -1075.262987 & - \\
\hline 4 & 0.361378 & 0.382271 & 0.383215 & 0.311544 & -1075.583212 & -1075.271668 & - \\
\hline 5 & 0.493944 & 0.52441 & 0.525354 & 0.429608 & -1874.907865 & -1874.478257 & - \\
\hline 6 & 0.493018 & 0.523724 & 0.524668 & 0.429662 & -1874.914506 & -1874.484844 & - \\
\hline${ }^{3}$ INT1 & 0.530405 & 0.596201 & 0.597146 & 0.422742 & -6115.012882 & -6114.59014 & - \\
\hline${ }^{5}$ INT1 & 0.529021 & 0.59571 & 0.596654 & 0.418063 & -6115.024677 & -6114.606614 & - \\
\hline${ }^{3} \mathrm{TS} 1$ & 0.529695 & 0.59514 & 0.596084 & 0.422548 & -6115.003068 & -6114.58052 & $-872.67 i$ \\
\hline${ }^{5}$ TS1 & 0.5284 & 0.594472 & 0.595416 & 0.419317 & -6115.002989 & -6114.583672 & $-405.68 \mathrm{i}$ \\
\hline${ }^{3}$ INT2 & 0.436979 & 0.495546 & 0.49649 & 0.338012 & -5585.424611 & -5585.086599 & - \\
\hline${ }^{5}$ INT2 & 0.437323 & 0.49592 & 0.496864 & 0.338511 & -5585.435305 & -5585.096794 & - \\
\hline${ }^{3} \mathrm{TS} 2 \mathrm{a}$ & 0.790456 & 0.869775 & 0.870719 & 0.669408 & -6585.763125 & -6585.093717 & $-1129.05 \mathrm{i}$ \\
\hline${ }^{5}$ TS2a & 0.790167 & 0.86961 & 0.870554 & 0.668394 & -6585.756213 & -6585.087819 & $-1632.34 \mathrm{i}$ \\
\hline${ }^{1}$ TS2b & 0.792989 & 0.871563 & 0.872507 & 0.675283 & -6585.761028 & -6585.085745 & $-847.05 i$ \\
\hline${ }^{3} \mathrm{TS} 2 \mathrm{~b}$ & 0.79541 & 0.873773 & 0.874717 & 0.677763 & -6585.781591 & -6585.103828 & $-97.82 \mathrm{i}$ \\
\hline${ }^{5} \mathrm{TS} 2 \mathrm{~b}$ & 0.794299 & 0.872895 & 0.873839 & 0.675574 & -6585.77425 & -6585.098676 & $-254.05 \mathrm{i}$ \\
\hline${ }^{3}$ INT3a & 0.795568 & 0.875477 & 0.876421 & 0.673594 & -6585.733019 & -6585.059425 & - \\
\hline${ }^{5}$ INT3a & 0.795688 & 0.875718 & 0.876662 & 0.672921 & -6585.766613 & -6585.093692 & - \\
\hline${ }^{3}$ INT3b & 0.795232 & 0.875309 & 0.876253 & 0.673859 & -6585.814084 & -6585.140225 & - \\
\hline${ }^{5}$ INT3b & 0.795078 & 0.875196 & 0.87614 & 0.67298 & -6585.818819 & -6585.145839 & - \\
\hline${ }^{5}$ TS3a & 0.794881 & 0.874566 & 0.87551 & 0.672715 & -6585.755384 & -6585.082669 & $-55.69 \mathrm{i}$ \\
\hline${ }^{5} \mathrm{TS} 3 \mathrm{~b}$ & 0.794296 & 0.873989 & 0.874933 & 0.67308 & -6585.817418 & -6585.144338 & $-173.48 \mathrm{i}$ \\
\hline
\end{tabular}




\begin{tabular}{|c|c|c|c|c|c|c|c|}
\hline${ }^{1}$ TS4 & 0.797771 & 0.8765 & 0.877444 & 0.678489 & - & - & $-418.75 \mathrm{i}$ \\
\hline${ }^{3}$ TS4 & -6585.782 & 0.797424 & 0.876357 & 0.877301 & 0.675872 & 1.553173 & $-166.6 \mathrm{i}$ \\
\hline${ }^{5} \mathrm{TS} 4$ & 0.796991 & 0.876103 & 0.877047 & 0.675475 & -6585.774455 & -6585.09898 & $-125.73 i$ \\
\hline${ }^{3}$ INT4a & 0.796014 & 0.875359 & 0.876303 & 0.673696 & -6585.792318 & -6585.118622 & - \\
\hline${ }^{5}$ INT4a & 0.798477 & 0.877592 & 0.878536 & 0.676254 & -6585.7993 & -6585.123046 & - \\
\hline${ }^{3}$ INT4 & 0.797234 & 0.875939 & 0.876883 & 0.677995 & -6585.809161 & -6585.131166 & - \\
\hline${ }^{5}$ INT4 & 0.796036 & 0.875305 & 0.876249 & 0.675008 & -6585.803231 & -6585.128223 & - \\
\hline${ }^{3}$ TS5 & 0.794934 & 0.872829 & 0.873773 & 0.67757 & -6585.804152 & -6585.126582 & $-411.31 \mathrm{i}$ \\
\hline${ }^{5}$ TS5 & 0.795241 & 0.873537 & 0.874482 & 0.676287 & -6585.80225 & -6585.125963 & $-26.14 \mathrm{i}$ \\
\hline${ }^{3}$ INT5 & 0.803421 & 0.881479 & 0.882423 & 0.686123 & -6585.855235 & -6585.169112 & - \\
\hline${ }^{5}$ INT5 & 0.799577 & 0.878204 & 0.879148 & 0.681454 & -6585.864712 & -6585.183258 & - \\
\hline${ }^{3}$ TS6 & 0.799663 & 0.877502 & 0.878446 & 0.682727 & -6585.850555 & -6585.167828 & $-993.11 \mathrm{i}$ \\
\hline${ }^{5}$ TS6 & 0.795112 & 0.873576 & 0.87452 & 0.676933 & -6585.861338 & -6585.184405 & $-1146.72 \mathrm{i}$ \\
\hline${ }^{3}$ INT6 & 0.662986 & 0.738604 & 0.739549 & 0.54557 & -6914.340475 & -6913.794905 & - \\
\hline${ }^{5}$ INT6 & 0.660229 & 0.736709 & 0.737653 & 0.539493 & -6914.361739 & -6913.822246 & - \\
\hline${ }^{3}$ TS7 & 0.660413 & 0.73563 & 0.736574 & 0.544268 & -6914.32883 & -6913.784562 & $-703.92 \mathrm{i}$ \\
\hline${ }^{5}$ TS7 & 0.660318 & 0.736043 & 0.736987 & 0.541441 & -6914.332885 & -6913.791444 & $-109.21 \mathrm{i}$ \\
\hline${ }^{3}$ INT7 & 0.568299 & 0.637223 & 0.638167 & 0.456113 & -6384.776634 & -6384.320521 & - \\
\hline 5INT7 & 0.568132 & 0.637105 & 0.638049 & 0.455324 & -6384.768862 & -6384.313538 & - \\
\hline${ }^{3} \mathrm{TS} 8 \mathrm{a}$ & 0.922589 & 1.011619 & 1.012563 & 0.791588 & -7385.099043 & -7384.307455 & $-1277.97 i$ \\
\hline${ }^{5}$ TS8a & 0.922186 & 1.0114 & 1.012345 & 0.789627 & -7385.094573 & -7384.304946 & $-1576.53 \mathrm{i}$ \\
\hline${ }^{1}$ TS8b & 0.92214 & 1.010912 & 1.011856 & 0.794481 & - & - & $-1952.17 \mathrm{i}$ \\
\hline${ }^{3} \mathrm{TS} 8 \mathrm{~b}$ & 0.924133 & 1.012758 & 1.013703 & 0.795295 & -7385.109494 & -7384.314199 & $-1570.25 \mathrm{i}$ \\
\hline${ }^{5} \mathrm{TS} 8 \mathrm{~b}$ & 0.92394 & 1.01268 & 1.013624 & 0.794449 & -7385.106036 & -7384.311587 & $-1258.38 \mathrm{i}$ \\
\hline${ }^{3}$ INT8a & 0.927925 & 1.0174 & 1.018344 & 0.795764 & -7385.105553 & -7384.309789 & - \\
\hline${ }^{5}$ INT8a & 0.928175 & 1.017549 & 1.018493 & 0.796081 & -7385.10478 & -7384.308699 & - \\
\hline${ }^{3}$ INT8b & 0.92968 & 1.019414 & 1.020359 & 0.798471 & -7385.155527 & -7384.357056 & - \\
\hline${ }^{5}$ INT8b & 0.926816 & 1.0169 & 1.017844 & 0.795356 & -7385.157193 & -7384.361837 & - \\
\hline${ }^{3} \mathrm{TS} 9 \mathrm{a}$ & 0.927502 & 1.016481 & 1.017425 & 0.797286 & -7385.068006 & -7384.27072 & $-297.53 \mathrm{i}$ \\
\hline${ }^{5}$ TS9a & 0.927559 & 1.016505 & 1.017449 & 0.796263 & -7385.092841 & -7384.296578 & $-216.23 \mathrm{i}$ \\
\hline
\end{tabular}




\begin{tabular}{|c|c|c|c|c|c|c|c|}
\hline${ }^{3} \mathrm{TS} 9 \mathrm{~b}$ & 0.928723 & 1.017095 & 1.018039 & 0.801615 & -7385.154395 & -7384.35278 & $-25.49 \mathrm{i}$ \\
\hline${ }^{5}$ TS9b & 0.926758 & 1.016091 & 1.017035 & 0.795548 & -7385.15728 & -7384.361732 & $-25.99 \mathrm{i}$ \\
\hline${ }^{1}$ TS10 & 0.929524 & 1.017544 & 1.018488 & 0.803985 & - & - & $-374.00 \mathrm{i}$ \\
\hline${ }^{3} \mathrm{TS} 10$ & 0.930113 & 1.017954 & 1.018898 & 0.803186 & -7385.10922 & -7384.306034 & $-269.54 \mathrm{i}$ \\
\hline${ }^{5}$ TS10 & 0.929972 & 1.017895 & 1.018839 & 0.802696 & -7385.109071 & -7384.306375 & $-212.42 \mathrm{i}$ \\
\hline${ }^{3}$ INT9 & 0.931429 & 1.019588 & 1.020533 & 0.802652 & -7385.144026 & -7384.341374 & - \\
\hline${ }^{5}$ INT9 & 0.92975 & 1.018899 & 1.019843 & 0.797073 & -7385.157523 & -7384.36045 & - \\
\hline${ }^{3}$ TS11 & 0.928008 & 1.015955 & 1.0169 & 0.798947 & -7385.126214 & -7384.327267 & $-903.38 \mathrm{i}$ \\
\hline${ }^{5}$ TS11 & 0.925311 & 1.013477 & 1.014421 & 0.795489 & -7385.133816 & -7384.338327 & $-487.98 \mathrm{i}$ \\
\hline${ }^{3}$ INT10 & 0.935134 & 1.022529 & 1.023473 & 0.809323 & -7385.177281 & -7384.367958 & - \\
\hline${ }^{5}$ INT10 & 0.931622 & 1.019598 & 1.020542 & 0.804381 & -7385.184202 & -7384.379821 & - \\
\hline${ }^{3} \mathrm{TS} 12$ & 0.930563 & 1.017699 & 1.018643 & 0.804719 & -7385.16483 & -7384.360111 & $-1132.55 \mathrm{i}$ \\
\hline${ }^{5} \mathrm{TS} 12$ & 0.928309 & 1.01598 & 1.016925 & 0.801347 & -7385.181531 & -7384.380184 & $-218.49 \mathrm{i}$ \\
\hline${ }^{3}$ TS2b-m1 & 0.563264 & 0.628434 & 0.629379 & 0.457609 & -5857.132719 & -5856.67511 & $-929.51 \mathrm{i}$ \\
\hline${ }^{5} \mathrm{TS} 2 \mathrm{~b}-\mathrm{m} 1$ & 0.563251 & 0.628465 & 0.62941 & 0.457102 & -5857.123989 & -5856.666887 & $-835.20 \mathrm{i}$ \\
\hline${ }^{3}$ TS4-m1 & 0.567819 & 0.63352 & 0.634465 & 0.460407 & -5857.130645 & -5856.670238 & $-275.16 \mathrm{i}$ \\
\hline${ }^{5} \mathrm{TS} 4-\mathrm{m} 1$ & 0.567458 & 0.633382 & 0.634326 & 0.458913 & -5857.122643 & -5856.66373 & $-145.94 i$ \\
\hline${ }^{3} \mathrm{TS} 2 \mathrm{~b}-\mathrm{m} 2$ & 0.634035 & 0.675111 & 0.676056 & 0.559796 & -3675.710476 & -3675.15068 & $-408.61 \mathrm{i}$ \\
\hline${ }^{5} \mathrm{TS} 2 \mathrm{~b}-\mathrm{m} 2$ & 0.632725 & 0.673932 & 0.674876 & 0.557937 & -3675.702637 & -3675.1447 & $-886.27 \mathrm{i}$ \\
\hline${ }^{3} \mathrm{TS} 4-\mathrm{m} 2$ & 0.638 & 0.67896 & 0.679905 & 0.564936 & -3675.71171 & -3675.146774 & $-236.70 \mathrm{i}$ \\
\hline${ }^{5}$ TS4-m2 & 0.637346 & 0.67855 & 0.679494 & 0.562988 & -3675.703208 & -3675.14022 & $-218.20 \mathrm{i}$ \\
\hline
\end{tabular}

ZPVE = zero-point vibrational energy; $\mathbf{T C E}=$ thermal correction to energy; $\mathbf{T C H}=$ thermal correction to enthalpy; TCG $=$ thermal correction to Gibbs free energy.

\section{Cartesian coordinates of the structures}

\section{Structure: 1}

$\begin{array}{llll}\text { C } & 3.533423 & 0.144604 & 0.292538 \\ \mathrm{C} & 2.732500 & 1.253838 & 0.182572 \\ \mathrm{C} & 1.330247 & 1.113069 & 0.019277\end{array}$




\begin{tabular}{|c|c|c|c|}
\hline C & 0.750429 & -0.197977 & -0.038730 \\
\hline C & 1.627767 & -1.313117 & 0.086361 \\
\hline C & 2.985615 & -1.157250 & 0.250437 \\
\hline $\mathrm{H}$ & 3.179159 & 2.242608 & 0.228099 \\
\hline C & 0.484756 & 2.247665 & -0.084674 \\
\hline C & -0.666403 & -0.341930 & -0.208473 \\
\hline $\mathrm{H}$ & 1.226949 & -2.319612 & 0.052642 \\
\hline $\mathrm{H}$ & 3.637913 & -2.017081 & 0.335731 \\
\hline C & -1.457884 & 0.799541 & -0.305313 \\
\hline C & -0.869849 & 2.088883 & -0.241545 \\
\hline O & 4.891050 & 0.364780 & 0.533773 \\
\hline C & 7.214126 & 0.116224 & 0.263597 \\
\hline $\mathrm{H}$ & 7.968085 & -0.424592 & -0.309090 \\
\hline $\mathrm{H}$ & 7.340086 & 1.195133 & 0.125415 \\
\hline $\mathrm{H}$ & 7.331182 & -0.091833 & 1.332195 \\
\hline C & 5.839472 & -0.304413 & -0.195894 \\
\hline O & 5.593247 & -1.100622 & -1.067676 \\
\hline C & -2.942547 & 0.637793 & -0.521556 \\
\hline C & -3.489707 & -0.624940 & 0.176006 \\
\hline C & -3.937441 & 1.751608 & -0.137229 \\
\hline $\mathrm{H}$ & -3.882901 & 2.615130 & -0.807167 \\
\hline C & -5.301750 & 1.024563 & -0.222704 \\
\hline $\mathrm{H}$ & -5.783385 & 1.171554 & -1.197271 \\
\hline C & -4.988996 & -0.480088 & -0.080713 \\
\hline O & -5.796406 & -1.379968 & -0.159586 \\
\hline C & -3.321082 & -0.599016 & 1.716630 \\
\hline $\mathrm{H}$ & -3.720190 & -1.527000 & 2.139444 \\
\hline $\mathrm{H}$ & -2.268485 & -0.506502 & 1.998592 \\
\hline $\mathrm{H}$ & -3.859549 & 0.234625 & 2.179866 \\
\hline $\mathrm{H}$ & -6.028407 & 1.335147 & 0.535359 \\
\hline $\mathrm{H}$ & -3.741205 & 2.115129 & 0.877476 \\
\hline C & -2.796544 & -1.837822 & -0.436392 \\
\hline C & -1.266464 & -1.747117 & -0.21784 \\
\hline $\mathrm{H}$ & -3.021162 & -1.864820 & -1.510015 \\
\hline $\mathrm{H}$ & -3.182937 & -2.771267 & -0.012971 \\
\hline $\mathrm{H}$ & -0.765828 & -2.354605 & -0.982263 \\
\hline H & -1.014161 & -2.221832 & 0.740705 \\
\hline $\mathrm{H}$ & -1.510774 & 2.962705 & -0.318264 \\
\hline $\mathrm{H}$ & 0.924500 & 3.240768 & -0.035774 \\
\hline $\mathrm{H}$ & -3.090036 & 0.468118 & -1.602900 \\
\hline
\end{tabular}

\section{Structure: ${ }^{12}$}




\begin{tabular}{|c|c|c|c|}
\hline $\mathrm{C}$ & -1.098599 & -2.840546 & 0.087370 \\
\hline C & -0.677733 & -4.217332 & 0.108663 \\
\hline C & 0.680146 & -4.216016 & 0.128110 \\
\hline C & 1.098483 & -2.838265 & 0.134546 \\
\hline $\mathrm{N}$ & -0.000995 & -2.003250 & 0.114047 \\
\hline $\mathrm{H}$ & -1.338045 & -5.071871 & 0.112552 \\
\hline $\mathrm{H}$ & 1.341674 & -5.069507 & 0.137243 \\
\hline C & 2.831046 & 1.102690 & 0.196698 \\
\hline C & 4.205871 & 0.684970 & 0.288547 \\
\hline C & 4.207770 & -0.673199 & 0.288695 \\
\hline C & 2.834118 & -1.094780 & 0.196897 \\
\hline $\mathrm{N}$ & 1.997602 & 0.002781 & 0.156012 \\
\hline $\mathrm{H}$ & 5.055586 & 1.347832 & 0.359470 \\
\hline $\mathrm{H}$ & 5.059327 & -1.333673 & 0.359752 \\
\hline C & -2.843273 & 1.094754 & 0.025821 \\
\hline C & -4.217875 & 0.673208 & -0.051009 \\
\hline $\mathrm{C}$ & -4.215977 & -0.684884 & -0.051156 \\
\hline C & -2.840205 & -1.102609 & 0.025593 \\
\hline $\mathrm{N}$ & -2.006941 & -0.002761 & 0.072700 \\
\hline $\mathrm{H}$ & -5.070730 & 1.333149 & -0.109197 \\
\hline $\mathrm{H}$ & -5.067000 & -1.347170 & -0.109536 \\
\hline C & -1.106564 & 2.837561 & 0.087828 \\
\hline $\mathrm{C}$ & -0.689482 & 4.215499 & 0.109296 \\
\hline C & 0.668399 & 4.217915 & 0.128453 \\
\hline C & 1.090523 & 2.841323 & 0.134599 \\
\hline $\mathrm{N}$ & -0.006671 & 2.003287 & 0.114184 \\
\hline $\mathrm{H}$ & -1.352135 & 5.068224 & 0.113466 \\
\hline $\mathrm{H}$ & 1.327580 & 5.073217 & 0.137611 \\
\hline $\mathrm{C}$ & -2.432257 & -2.436029 & 0.038925 \\
\hline C & 2.424374 & 2.436336 & 0.171282 \\
\hline C & 2.431202 & -2.429571 & 0.171540 \\
\hline $\mathrm{C}$ & -2.439096 & 2.429325 & 0.039421 \\
\hline Mn & 0.002539 & 0.000032 & -0.165289 \\
\hline $\mathrm{Cl}$ & 0.035395 & 0.000167 & -2.367894 \\
\hline $\mathrm{C}$ & 3.489647 & -3.479785 & 0.191938 \\
\hline C & 4.331208 & -3.681852 & -0.906646 \\
\hline C & 3.683786 & -4.297265 & 1.309322 \\
\hline C & 5.327368 & -4.655902 & -0.898568 \\
\hline C & 4.668243 & -5.282056 & 1.338102 \\
\hline C & 5.493443 & -5.459074 & 0.228601 \\
\hline C & -3.484797 & -3.491583 & -0.003066 \\
\hline C & -4.335734 & -3.711737 & 1.084273 \\
\hline C & -3.660942 & -4.298908 & -1.131218 \\
\hline & -5.326112 & -4.690655 & 1.05654 \\
\hline
\end{tabular}




\begin{tabular}{|c|c|c|c|}
\hline C & -4.639965 & -5.288988 & -1.179637 \\
\hline C & -5.475652 & -5.482636 & -0.080828 \\
\hline $\mathrm{C}$ & -3.494628 & 3.481897 & -0.002219 \\
\hline $\mathrm{C}$ & -4.346216 & 3.699288 & 1.085174 \\
\hline C & -3.673137 & 4.289012 & -1.130152 \\
\hline C & -5.339439 & 4.675322 & 1.057697 \\
\hline $\mathrm{C}$ & -4.655027 & 5.276263 & -1.178305 \\
\hline $\mathrm{C}$ & -5.491291 & 5.467171 & -0.079460 \\
\hline $\mathrm{C}$ & 3.479892 & 3.489510 & 0.191322 \\
\hline $\mathrm{C}$ & 3.672118 & 4.307634 & 1.308562 \\
\hline $\mathrm{C}$ & 4.320502 & 3.693811 & -0.907571 \\
\hline C & 4.653828 & 5.295172 & 1.336923 \\
\hline C & 5.313945 & 4.670641 & -0.899912 \\
\hline $\mathrm{C}$ & 5.478154 & 5.474384 & 0.227120 \\
\hline $\mathrm{F}$ & -4.785972 & -6.048215 & -2.269762 \\
\hline $\mathrm{F}$ & -2.870178 & -4.135012 & -2.198131 \\
\hline $\mathrm{F}$ & -6.418431 & -6.425959 & -0.117346 \\
\hline $\mathrm{F}$ & -6.127038 & -4.878220 & 2.110213 \\
\hline $\mathrm{F}$ & -4.213675 & -2.962462 & 2.188088 \\
\hline $\mathrm{F}$ & -2.881938 & 4.127681 & -2.197136 \\
\hline $\mathrm{F}$ & -4.803222 & 6.035344 & -2.268234 \\
\hline $\mathrm{F}$ & -6.436830 & 6.407736 & -0.115730 \\
\hline $\mathrm{F}$ & -6.140928 & 4.860255 & 2.111407 \\
\hline $\mathrm{F}$ & -4.222015 & 2.950061 & 2.188782 \\
\hline $\mathrm{F}$ & 2.903193 & -4.147650 & 2.388202 \\
\hline $\mathrm{F}$ & 4.194976 & -2.921727 & -1.998997 \\
\hline $\mathrm{F}$ & 6.119170 & -4.827444 & -1.961265 \\
\hline $\mathrm{F}$ & 6.442246 & -6.396862 & 0.245935 \\
\hline $\mathrm{F}$ & 4.830068 & -6.050986 & 2.419676 \\
\hline $\mathrm{F}$ & 2.892255 & 4.155977 & 2.387686 \\
\hline $\mathrm{F}$ & 4.185999 & 2.933225 & -1.999808 \\
\hline $\mathrm{F}$ & 6.104907 & 4.844289 & -1.962895 \\
\hline $\mathrm{F}$ & 6.424338 & 6.414824 & 0.244041 \\
\hline $\mathrm{F}$ & 4.813874 & 6.064664 & 2.41836 \\
\hline
\end{tabular}

\section{Structure: ${ }^{3} 2$}

C
C
C
C
N
H
H

$$
\begin{array}{rrr}
0.807603 & -2.933949 & -0.144487 \\
0.255292 & -4.264183 & -0.132076 \\
-1.096101 & -4.131207 & -0.114849 \\
-1.378398 & -2.718977 & -0.099693 \\
-0.203772 & -1.994164 & -0.131695 \\
0.829048 & -5.179083 & -0.135928 \\
-1.837453 & -4.916579 & -0.114965
\end{array}
$$


C

$\mathrm{Mn}$

$\mathrm{Cl}$

C

C

C

C

C

C

C

C

C

C

C

C

C

C

C

C

C

\begin{tabular}{|c|c|c|}
\hline-2.719850 & 1.371412 & -0.028088 \\
\hline-4.129896 & 1.090566 & 0.057638 \\
\hline-4.264829 & -0.260825 & 055440 \\
\hline & -0.814409 & \\
\hline 1.998550 & 0.195655 & -0 . \\
\hline 4.91177 & 1.8327 & \\
\hline-5 & -0.8342 & \\
\hline & & \\
\hline & & \\
\hline & -1.09 & -0.2 \\
\hline & $-1 \cdot 3$ & -0.2 \\
\hline & -0.1 & -0.1 \\
\hline & & -0 \\
\hline & $-1 . \varepsilon$ & -0 \\
\hline 1. & 2.7 & -0 \\
\hline & & -0 \\
\hline-0. & 4.2 & -0 . \\
\hline$-0 . \varepsilon$ & 2.9 & -0 . \\
\hline & & -0 . \\
\hline 1.8 & 4.9 & -0 . \\
\hline-0 . & 5.1 & -0 . \\
\hline & -2.6 & -0 . \\
\hline-2.18 & 2.6 & -0.0 \\
\hline$-2 \cdot 6$ & -2.1 & -0.0 \\
\hline 2. & & -0 \\
\hline & -0.0 & \\
\hline 0.0 & -0.0 & 2 \\
\hline 3.8 & 3.1 & -0.1 \\
\hline & & \\
\hline 4. & 3. & -1 \\
\hline 5 & 4.1 & \\
\hline 5.1 & & $-1 \cdot 3$ \\
\hline 5. & & -0.2 \\
\hline 3 & -3.8 & -0.1 \\
\hline 3.9 & -4.0 & \\
\hline 3.2 & -4.6 & $-1 \cdot 3$ \\
\hline 4.82 & -5.15 & 0.9 \\
\hline 4.12 & -5.724933 & -1.319002 \\
\hline 4.920269 & -5.977 & -0.202337 \\
\hline-3.818019 & -3.126594 & -0.003733 \\
\hline-4.690233 & -3.259033 & -1.088333 \\
\hline-4.070761 & -3.9134 & 1.124 \\
\hline-5.773832 & -4.1 & -1.0 \\
\hline-5.14 & $-4 . \varepsilon$ & 1 \\
\hline
\end{tabular}


C
C
C
C
C
C
C
F
F
F
F
F
F
F
F
F
F
F
F
F
F
F
F
F
F
F
F

F

$\begin{array}{rrr}-5.998548 & -4.907801 & 0.079226 \\ -3.127500 & 3.811263 & 0.009594 \\ -3.955198 & 4.118157 & -1.074567 \\ -3.222280 & 4.625848 & 1.142082 \\ -4.845690 & 5.188569 & -1.039858 \\ -4.100316 & 5.706154 & 1.197577 \\ -4.915204 & 5.985662 & 0.101635 \\ 2.489327 & -4.425740 & -2.387490 \\ 4.217738 & -6.511359 & -2.396156 \\ 5.771518 & -7.005174 & -0.208606 \\ 5.589552 & -5.401029 & 1.989859 \\ 3.294167 & 3.857518 & -2.390275 \\ 5.395929 & 5.566097 & -2.412034 \\ 7.032552 & 5.743963 & -0.236295 \\ 6.557534 & 4.202579 & 1.963256 \\ 4.458202 & 2.491950 & 1.991490 \\ 3.863534 & -3.313587 & 2.004837 \\ -2.449382 & 4.380300 & 2.206478 \\ -3.908327 & 3.366154 & -2.182262 \\ -4.169987 & 6.470448 & 2.291785 \\ -5.626862 & 5.458236 & -2.090566 \\ -5.761893 & 7.015915 & 0.145110 \\ -3.263981 & -3.830760 & 2.188800 \\ -5.361702 & -5.542293 & 2.265352 \\ -7.030967 & -5.751982 & 0.118236 \\ -6.592934 & -4.238699 & -2.109179 \\ -4.497354 & -2.524789 & -2.192071\end{array}$

\section{Structure:}

$\mathrm{C}$
$\mathrm{C}$
$\mathrm{C}$
$\mathrm{C}$
$\mathrm{N}$
$\mathrm{H}$
$\mathrm{H}$
$\mathrm{C}$
$\mathrm{C}$
$\mathrm{C}$
$\mathrm{C}$
$\mathrm{N}$
$\mathrm{H}$
$\mathrm{H}$

$-2.846216$

$-1.102192$

$-0.028239$

$-4.220216$

$-0.679623$

0.048369

$-4.220354$

0.678818

0.048211

$-2.846437$

1.101650

$-0.028472$

$-2.018917$

$-0.000192$

$-0.076723$

$-5.072113$

$-1.340552$

0.106999

$-5.072386$

1.339585

0.106689

1.097824

2. 844965

$-0.136374$

0.674237

4.220904

$-0.133638$

$-0.684003$

4.22012

$-0.114889$

$-1.105749$

2. 843934

$-0.089808$

$-0.003553$

2.015706

$-0.114378$

1.334166

5.075445

$-0.144765$

$-1.345479$

5.073606

$-0.121631$ 


\begin{tabular}{|c|c|c|c|}
\hline $\mathrm{C}$ & 1.098381 & -2.844712 & -0.136610 \\
\hline C & 0.675074 & -4.220736 & -0.133808 \\
\hline C & -0.683163 & -4.220231 & -0.114731 \\
\hline C & -1.105182 & -2.844127 & -0.089575 \\
\hline $\mathrm{N}$ & -0.003158 & -2.015676 & -0.114370 \\
\hline $\mathrm{H}$ & 1.335175 & -5.075142 & -0.145088 \\
\hline $\mathrm{H}$ & -1.344467 & -5.073848 & -0.121318 \\
\hline C & 2.837887 & -1.101681 & -0.196404 \\
\hline $\mathrm{C}$ & 4.210595 & -0.678822 & -0.292732 \\
\hline $\mathrm{C}$ & 4.210465 & 0.679710 & -0.292563 \\
\hline C & 2.837673 & 1.102279 & -0.196168 \\
\hline $\mathrm{N}$ & 2.010438 & 0.000215 & -0.151863 \\
\hline $\mathrm{H}$ & 5.061012 & -1.340225 & -0.367172 \\
\hline $\mathrm{H}$ & 5.060751 & 1.341300 & -0.366843 \\
\hline C & -2.439031 & -2.436907 & -0.040985 \\
\hline $\mathrm{C}$ & 2.431919 & 2.437251 & -0.172433 \\
\hline C & -2.439522 & 2.436447 & -0.041383 \\
\hline $\mathrm{C}$ & 2.432392 & -2.436734 & -0.172838 \\
\hline $\mathrm{Mn}$ & 0.001982 & 0.000013 & 0.171549 \\
\hline $\mathrm{Cl}$ & 0.028599 & 0.000046 & 2.513339 \\
\hline $\mathrm{C}$ & -3.493429 & 3.490049 & -0.000945 \\
\hline $\mathrm{C}$ & -4.349699 & 3.702104 & -1.085968 \\
\hline $\mathrm{C}$ & -3.666023 & 4.303833 & 1.123552 \\
\hline $\mathrm{C}$ & -5.342188 & 4.678734 & -1.059628 \\
\hline C & -4.647131 & 5.292097 & 1.169943 \\
\hline C & -5.488262 & 5.477136 & 0.073768 \\
\hline C & -3.492712 & -3.490723 & -0.000272 \\
\hline C & -3.664943 & -4.304429 & 1.124337 \\
\hline C & -4.349116 & -3.703077 & -1.085132 \\
\hline $\mathrm{C}$ & -4.645834 & -5.292896 & 1.170994 \\
\hline C & -5.341394 & -4.679913 & -1.058524 \\
\hline C & -5.487110 & -5.478228 & 0.074980 \\
\hline $\mathrm{C}$ & 3.489250 & -3.487826 & -0.196569 \\
\hline C & 4.337378 & -3.687478 & 0.897833 \\
\hline $\mathrm{C}$ & 3.675882 & -4.308737 & -1.312968 \\
\hline C & 5.332234 & -4.662969 & 0.886088 \\
\hline C & 4.658558 & -5.295037 & -1.344797 \\
\hline C & 5.490178 & -5.469815 & -0.239667 \\
\hline C & 3.488594 & 3.488531 & -0.195873 \\
\hline C & 3.675236 & 4.309642 & -1.312123 \\
\hline C & 4.336570 & 3.688132 & 0.898656 \\
\hline $\mathrm{C}$ & 4.657767 & 5.296095 & -1.343682 \\
\hline C & 5.331280 & 4.663775 & 0.887180 \\
\hline $\mathrm{C}$ & 5.489234 & 5.470822 & -0.23843 \\
\hline
\end{tabular}




$\begin{array}{lrrr}F & -4.230550 & 2.946606 & -2.186065 \\ F & -6.147988 & 4.858313 & -2.110873 \\ F & -6.432806 & 6.418447 & 0.108767 \\ F & -4.789903 & 6.057666 & 2.255672 \\ F & -2.870194 & 4.148486 & 2.187018 \\ F & -2.868968 & -4.148803 & 2.187651 \\ F & -4.230313 & -2.947669 & -2.185329 \\ F & -4.788264 & -6.058381 & 2.256828 \\ F & -6.431451 & -6.419733 & 0.110235 \\ F & -6.147333 & -4.859769 & -2.109615 \\ F & 4.208476 & 2.924255 & 1.988485 \\ F & 2.888682 & 4.161745 & -2.387128 \\ F & 4.812115 & 6.068313 & -2.423906 \\ F & 6.436392 & 6.409914 & -0.258583 \\ F & 6.129344 & 4.833081 & 1.945159 \\ F & 4.209284 & -2.923802 & 1.987805 \\ F & 2.889177 & -4.160793 & -2.387855 \\ F & 4.812896 & -6.067063 & -2.425159 \\ F & 6.437472 & -6.408764 & -0.260074 \\ F & 6.130440 & -4.832326 & 1.943951\end{array}$

\section{Structure: 3}

$\begin{array}{lrrr}\mathrm{C} & 3.601864 & 0.280380 & -0.304008 \\ \mathrm{C} & 2.802174 & 1.377978 & -0.110512 \\ \mathrm{C} & 1.389353 & 1.246449 & -0.130458 \\ \mathrm{C} & 0.795637 & -0.041530 & -0.345546 \\ \mathrm{C} & 1.674069 & -1.148312 & -0.531340 \\ \mathrm{C} & 3.041797 & -0.999349 & -0.519617 \\ \mathrm{H} & 3.256722 & 2.351045 & 0.049607 \\ \mathrm{C} & 0.548961 & 2.373038 & 0.061289 \\ \mathrm{C} & -0.634099 & -0.159809 & -0.384382 \\ \mathrm{H} & 1.259622 & -2.140675 & -0.650528 \\ \mathrm{H} & 3.693245 & -1.853513 & -0.654347 \\ \mathrm{C} & -1.421099 & 0.974704 & -0.205790 \\ \mathrm{C} & -0.816015 & 2.236459 & 0.023758 \\ \mathrm{O} & 4.979343 & 0.502744 & -0.360555 \\ \mathrm{C} & 7.258086 & 0.162199 & 0.109430 \\ \mathrm{H} & 7.942351 & -0.471937 & 0.673857 \\ \mathrm{H} & 7.489779 & 0.108480 & -0.959424 \\ \mathrm{H} & 7.374140 & 1.206808 & 0.416458 \\ \mathrm{C} & 5.839221 & -0.291893 & 0.352420 \\ \mathrm{O} & 5.496371 & -1.205994 & 1.060312 \\ \mathrm{C} & -2.919096 & 0.863293 & -0.340678\end{array}$


$-3.442144$

$-0.510218$

0.119665

$-3.873962$

1.880466

0.316475

$-3.847658$

2.859248

$-0.171934$

$-5.250651$

1.185900

0.166179

$-5.795332$

1.539419

$-0.717628$

$-4.948596$

$-0.313964$

$-0.039718$

$-5.766434$

$-1.173520$

$-0.285471$

$-3.212011$

$-0.790332$

1.627516

$-3.627064$

$-1.772507$

1.876497

$-2.148558$

$-0.799570$

1.870126

$-3.709433$

$-0.048985$

2. 262108

$-5.919746$

1. 323578

1. 022038

$-3.614954$

2.034701

1.369292

$-2.788087$

$-1.564982$

$-0.764312$

$-1.248219$

$-1.542304$

$-0.615495$

$-3.053975$

$-1.368001$

$-1.811425$

$-3.158796$

$-2.571808$

$-0.534860$

$-0.816101$

$-1.953404$

$-1.542976$

$-1.451338$

3.105585

0.167651

1.002632

3. 345661

0.235868

$-0.827117$

$-2.357026$

0.493883

$-1.178974$

$-3.250015$

0.348208

$-3.136977$

0.917211

$-1.423188$

\section{Structure: 4}

C
C
C
C
C
C
H
C
C
H
H
C
C
O
C
H
H
H

3.485456

2.702020

1.301062

0.695121

1.559260

2.919468

3.155787

0.464446

$-0.722890$

1.143538

3. 558615

$-1.493643$

$-0.894646$

4.846950

7.165262

7.910057

7.303667

7.284419
$-0.069337$

1.053188

0.924380

$-0.373023$

$-1.498132$

$-1.361667$

2.035762

2.073775

$-0.505385$

$-2.498375$

$-2.231555$

0.647853

1.930190

0.130226

$-0.141330$

$-0.693182$

0.935488

$-0.346819$
0.298081

0.189478

0.025658

$-0.035328$

0.088721

0.253668

0.235925

$-0.077757$

$-0.206019$

0.053080

0.337970

$-0.299870$

$-0.235653$

0.538897

0.254264

$-0.319939$

0.111516

1.323089 


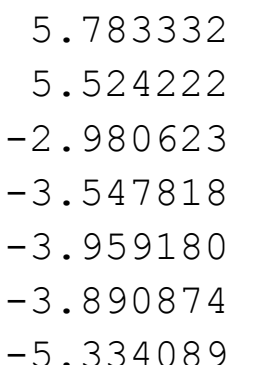

$-5.334089$

$-5.809457$

$-5.044316$

$-5.866183$

$-3.381646$

$-3.793300$

$-2.328094$

$-3.910266$

$-6.058722$

$-3.760076$

$-2.871687$

$-1.341113$

$-3.092132$

$-3.276206$

$-0.849633$

$-1.102705$

$-1.523194$

1.085776

0.418329

$-3.128524$
$-0.545993$

$-1.337077$

0.512391

$-0.745314$

1.639004

2.505347

0.933406

1.091338

$-0.576557$

$-1.462832$

$-0.728135$

$-1.652822$

$-0.650551$

0.110069

1.252236

1.993226

$-1.966339$

$-1.901956$

$-1.983911$

$-2.895022$

$-2.518390$

$-2.383607$

2.815114

3.290898

3.989108

0.350707
$-0.198183$

$-1.071265$

$-0.518030$

0.172817

$-0.129375$

$-0.794823$

$-0.222376$

$-1.198298$

$-0.085229$

$-0.167676$

1.713961

2.131854

1.996539

2.180515

0.534151

0.888100

$-0.442763$

$-0.216965$

$-1.517524$

$-0.025787$

$-0.980477$

0.742052

$-0.311614$

$-0.008316$

$-0.093616$

$-1.600440$

\section{Structure: 5}

C

C

C

C

C

C

$\mathrm{H}$

C

C

$\mathrm{H}$

$\mathrm{H}$

C

C

O

C

$$
\begin{array}{rrr}
3.175750 & -2.217325 & 0.622711 \\
2.358849 & -3.048996 & -0.105107 \\
0.964033 & -2.783159 & -0.168362 \\
0.407207 & -1.647856 & 0.507070 \\
1.296671 & -0.835774 & 1.270707 \\
2.642579 & -1.114160 & 1.327425 \\
2.762820 & -3.900033 & -0.636702 \\
0.100670 & -3.648653 & -0.889717 \\
-1.002370 & -1.392030 & 0.412409 \\
0.922641 & 0.012018 & 1.831091 \\
3.315635 & -0.502087 & 1.919690 \\
-1.819890 & -2.295934 & -0.262430 \\
-1.252328 & -3.419751 & -0.915742 \\
4.544526 & -2.408633 & 0.806515 \\
6.776152 & -2.965269 & 0.338536
\end{array}
$$


$\mathrm{H}$

$\mathrm{H}$

$\mathrm{H}$

C

O

C

C

C

$\mathrm{H}$

C

$\mathrm{H}$

C

O

C

$\mathrm{H}$

$\mathrm{H}$

$\mathrm{H}$

$\mathrm{H}$

$\mathrm{H}$

C

C

$\mathrm{H}$

$\mathrm{H}$

$\mathrm{H}$

$\mathrm{H}$

$\mathrm{H}$

S

O

0

C

C

C

C

$\mathrm{H}$

C

$\mathrm{H}$

C

$\mathrm{H}$

$\mathrm{H}$

C

$\mathrm{H}$

$\mathrm{H}$

$\mathrm{H}$

N

\begin{tabular}{|c|c|c|}
\hline 7.427758 & -3.357897 & -0.442620 \\
\hline 7.108229 & -1.966250 & 0.639298 \\
\hline 6.824896 & -3.605865 & \\
\hline 5.357 & -2.907062 & -0.17571 \\
\hline 4.989453 & -3.242242 & -1.27465 \\
\hline-3.317189 & -2.111827 & -0.217 \\
\hline 3.72122 & -0.625964 & -0.209 \\
\hline 4.25 & -2.759946 & -1.25 \\
\hline-4.31 & -3.846712 & -1.14 \\
\hline-5.60 & -2.058669 & -0.9 \\
\hline-6.22 & -2.6485 & -0.2 \\
\hline-5.24 & -0.741239 & -0.23 \\
\hline-6.04 & 0.047498 & \\
\hline-3.33 & 0.10 & -1.5 \\
\hline-3.51 & 1.17 & -1.4 \\
\hline-2.29 & -0.07 & -1.7 \\
\hline-3.94 & -0.23 & $-2 \cdot 3$ \\
\hline-6.20 & -1.85 & -1.8 \\
\hline-3.9 & -2.55 & $-2 \cdot 2$ \\
\hline-3.1 & 0.02 & \\
\hline-1.56 & -0.104 & 1.03 \\
\hline-3.50 & -0.48 & 1.9 \\
\hline-3.38 & $1.0^{-}$ & \\
\hline-1.225 & -0.037780 & 2.0 \\
\hline-1.90 & -4.103040 & -1.4 \\
\hline 0.52 & -4.50 & -1.4 \\
\hline-0.73 & 2.479425 & 1.1 \\
\hline-0.16 & 2.095 & 2.4 \\
\hline-1.96 & 3.29 & 1.1 \\
\hline 0.4 & 3.25 & \\
\hline 1.68 & 2.638548 & -0.13 \\
\hline 0.25 & 4.603101 & -0.21 \\
\hline 2.6 & 3.309178 & -0.8 \\
\hline 1.85 & 1.616 & 0.19 \\
\hline 1.226729 & 5.260193 & -0.988388 \\
\hline-0.683685 & 5.086877 & 0.02 \\
\hline 2.42 & 4.628159 & -1.324 \\
\hline 3.566962 & 2.802101 & -1.148 \\
\hline 1.044992 & 6.278634 & -1.321784 \\
\hline 3.489618 & 5.341195 & -2.129 \\
\hline 3.120688 & 6.288487 & -2.534 \\
\hline 4.368186 & 5.562625 & -1.51 \\
\hline & 4.724 & -2 \\
\hline-0.8 & 1.03 & 0 \\
\hline
\end{tabular}


$\mathrm{H}$

$\mathrm{H}$

$-1.284379$

$-3.643182$
1.226299

$-2.512034$
$-0.578758$

0.759568

Structure: 6

C

C

C

C

C

C

$\mathrm{H}$

C

C

$\mathrm{H}$

$\mathrm{H}$

C

C

O

C

$\mathrm{H}$

$\mathrm{H}$

$\mathrm{H}$

C

O

C

C

C

$\mathrm{H}$

C

$\mathrm{H}$

C

O

C

$\mathrm{H}$

$\mathrm{H}$

$\mathrm{H}$

$\mathrm{H}$

$\mathrm{H}$

C

C

$\mathrm{H}$

$\mathrm{H}$

$\mathrm{H}$

$\begin{array}{rrr}-3.835647 & -1.481611 & -0.360805 \\ -3.053370 & -0.480850 & 0.164264 \\ -1.678883 & -0.718534 & 0.414896 \\ -1.091818 & -1.985587 & 0.075363 \\ -1.949952 & -2.980040 & -0.474720 \\ -3.289208 & -2.742623 & -0.679477 \\ -3.482551 & 0.488631 & 0.362931 \\ -0.839090 & 0.285363 & 0.987022 \\ 0.313335 & -2.206495 & 0.260945 \\ -1.549332 & -3.952817 & -0.735166 \\ -3.941940 & -3.503578 & -1.094925 \\ 1.086022 & -1.197433 & 0.825592 \\ 0.496049 & 0.029536 & 1.197342 \\ -5.179093 & -1.322985 & -0.688753 \\ -7.408046 & -0.598669 & -0.521434 \\ -8.102693 & -0.071685 & 0.133211 \\ -7.362436 & -0.100463 & -1.495773 \\ -7.751961 & -1.623563 & -0.693222 \\ -6.037222 & -0.606833 & 0.108133 \\ -5.728209 & -0.073775 & 1.144758 \\ 2.560411 & -1.434043 & 1.028039 \\ 3.147760 & -2.282285 & -0.118230 \\ 3.538851 & -0.252630 & 1.179424 \\ 3.452555 & 0.242910 & 2.151238 \\ 4.916834 & -0.927806 & 0.979186 \\ 5.386473 & -1.196726 & 1.933233 \\ 5.4640082 & -2.237383 & 0.210336 \\ 3.002983 & -1.615782 & -1.510150 \\ 3.453239 & -2.262828 & -2.270146 \\ 1.951822 & -1.451201 & -1.762760 \\ 3.504639 & -0.643301 & -1.556763 \\ 5.645089 & -0.316669 & 0.434520 \\ 3.346510 & 0.505244 & 0.414408 \\ 2.472015 & -3.650204 & -0.079651 \\ 0.935763 & -3.506260 & -0.246511 \\ 2.701263 & -4.124052 & 0.882754 \\ 0.872261 & -4.311727 & -0.855410 \\ -14530 & -4.363552 & 0.235523\end{array}$




$\begin{array}{rrr}0.684562 & -3.592778 & -1.312413 \\ 1.106167 & 0.812633 & 1.633162 \\ -1.336250 & 2.756999 & 0.048663 \\ -1.833294 & 3.983394 & 0.678401 \\ -1.984019 & 2.219277 & -1.155225 \\ 0.413853 & 2.890951 & -0.296194 \\ 1.231232 & 3.607061 & 0.579908 \\ 0.940906 & 2.230534 & -1.404766 \\ 2.602896 & 3.643726 & 0.341886 \\ 0.793812 & 4.122038 & 1.429079 \\ 2.316126 & 2.281559 & -1.629324 \\ 0.279739 & 1.685141 & -2.069043 \\ 3.166602 & 2.975506 & -0.757364 \\ 3.248563 & 4.198100 & 1.018320 \\ 2.734670 & 1.768611 & -2.491514 \\ 4.661538 & 2.969630 & -0.969272 \\ 4.919514 & 2.816319 & -2.022019 \\ 5.119525 & 3.907152 & -0.637270 \\ 5.129091 & 2.158089 & -0.394905 \\ -2.213792 & 1.641627 & 1.787112 \\ -1.308575 & 1.599429 & 1.318678 \\ 2.673282 & -2.042843 & 1.941989\end{array}$

\section{Structure: ${ }^{3}$ INT1}

$\begin{array}{lrrr}\mathrm{C} & -2.345001 & 1.469065 & -0.592491 \\ \mathrm{C} & -3.764225 & 1.201100 & -0.666819 \\ \mathrm{C} & -3.906153 & -0.143123 & -0.797089 \\ \mathrm{C} & -2.574902 & -0.707095 & -0.796378 \\ \mathrm{~N} & -1.644866 & 0.292433 & -0.658294 \\ \mathrm{H} & -4.546776 & 1.942880 & -0.608415 \\ \mathrm{H} & -4.825796 & -0.705194 & -0.868730 \\ \mathrm{C} & 1.154645 & -2.868979 & -0.507674 \\ \mathrm{C} & 0.608836 & -4.190781 & -0.703172 \\ \mathrm{C} & -0.730059 & -4.042545 & -0.893663 \\ \mathrm{C} & -1.013510 & -2.631234 & -0.803423 \\ \mathrm{~N} & 0.148089 & -1.933801 & -0.569351 \\ \mathrm{H} & 1.181193 & -5.107102 & -0.719082 \\ \mathrm{H} & -1.458428 & -4.815807 & -1.091954 \\ \mathrm{C} & 1.766969 & 2.787230 & -0.329306 \\ \mathrm{C} & 1.494020 & 4.206966 & -0.292673 \\ \mathrm{C} & 0.144117 & 4.350331 & -0.326349 \\ \mathrm{C} & -0.417659 & 3.018864 & -0.389320 \\ \mathrm{~N} & 0.588705 & 2.088137 & -0.388058\end{array}$




\begin{tabular}{|c|c|c|c|}
\hline $\mathrm{H}$ & 2.240068 & 4.986533 & -0.240512 \\
\hline $\mathrm{H}$ & -0.422451 & 5.270312 & -0.325415 \\
\hline C & 3.317290 & 0.858698 & -0.253041 \\
\hline C & 4.642293 & 0.289484 & -0.126618 \\
\hline C & 4.489338 & -1.058516 & -0.094399 \\
\hline $\mathrm{C}$ & 3.072652 & -1.321455 & -0.232865 \\
\hline $\mathrm{N}$ & 2.383146 & -0.140583 & -0.316178 \\
\hline $\mathrm{H}$ & 5.563239 & 0.850841 & -0.068224 \\
\hline $\mathrm{H}$ & 5.260348 & -1.808575 & 0.004335 \\
\hline $\mathrm{C}$ & -1.787583 & 2.745894 & -0.457239 \\
\hline C & 2.512753 & -2.601322 & -0.303551 \\
\hline $\mathrm{C}$ & -2.295086 & -2.074794 & -0.880200 \\
\hline $\mathrm{C}$ & 3.048721 & 2.231295 & -0.278529 \\
\hline $\mathrm{Mn}$ & 0.374617 & 0.085649 & -0.537280 \\
\hline $\mathrm{Cl}$ & 0.587878 & 0.155472 & -2.787173 \\
\hline $\mathrm{C}$ & -2.556498 & 1.230610 & 2.689317 \\
\hline C & -3.793616 & 1.862847 & 2.846019 \\
\hline $\mathrm{C}$ & -4.968514 & 1.112538 & 2.922524 \\
\hline $\mathrm{C}$ & -4.924265 & -0.279435 & 2.823937 \\
\hline $\mathrm{C}$ & -3.701490 & -0.936334 & 2.661211 \\
\hline $\mathrm{C}$ & -2.550194 & -0.156289 & 2.617840 \\
\hline $\mathrm{H}$ & -1.641712 & 1.805890 & 2.597736 \\
\hline $\mathrm{H}$ & -3.823171 & 2.944373 & 2.913543 \\
\hline $\mathrm{H}$ & -5.922778 & 1.615143 & 3.050562 \\
\hline $\mathrm{H}$ & -5.839649 & -0.862994 & 2.861214 \\
\hline $\mathrm{H}$ & -3.662087 & -2.014126 & 2.553222 \\
\hline I & -0.643675 & -1.143359 & 2.439730 \\
\hline O & 0.270541 & 0.225868 & 1.398744 \\
\hline $\mathrm{C}$ & -3.450343 & -3.009472 & -0.992862 \\
\hline $\mathrm{C}$ & -3.811038 & -3.842750 & 0.069466 \\
\hline $\mathrm{C}$ & -4.233167 & -3.081844 & -2.149539 \\
\hline $\mathrm{C}$ & -4.897008 & -4.709605 & 0.002037 \\
\hline $\mathrm{C}$ & -5.327834 & -3.940275 & -2.243966 \\
\hline C & -5.658192 & -4.758247 & -1.164814 \\
\hline $\mathrm{F}$ & -5.216284 & -5.486131 & 1.044164 \\
\hline $\mathrm{F}$ & -3.101951 & -3.814081 & 1.218253 \\
\hline $\mathrm{F}$ & -3.945957 & -2.305612 & -3.198308 \\
\hline $\mathrm{F}$ & -6.059098 & -3.988092 & -3.361160 \\
\hline $\mathrm{F}$ & -6.704013 & -5.583639 & -1.245483 \\
\hline C & 4.206839 & 3.170261 & -0.228681 \\
\hline $\mathrm{C}$ & 4.978249 & 3.320419 & 0.927397 \\
\hline $\mathrm{C}$ & 4.560498 & 3.942533 & -1.339741 \\
\hline $\mathrm{C}$ & 6.061958 & 4.193676 & 0.981864 \\
\hline $\mathrm{F}$ & 4.686309 & 2.604761 & 2.02272 \\
\hline
\end{tabular}




$\begin{array}{lrrr}C & 5.634992 & 4.829123 & -1.306663 \\ F & 3.853221 & 3.846868 & -2.470981 \\ C & 6.388679 & 4.952000 & -0.140890 \\ F & 6.783920 & 4.314543 & 2.102003 \\ F & 5.949864 & 5.557437 & -2.383042 \\ F & 7.422655 & 5.796086 & -0.099105 \\ C & 3.421197 & -3.773844 & -0.151851 \\ C & 3.356578 & -4.582869 & 0.986463 \\ C & 4.358145 & -4.119880 & -1.130049 \\ C & 4.182197 & -5.691122 & 1.153896 \\ F & 2.472875 & -4.298978 & 1.957557 \\ C & 5.201346 & -5.219818 & -0.982589 \\ F & 4.467059 & -3.382978 & -2.240978 \\ C & 5.109784 & -6.008504 & 0.162933 \\ F & 4.095086 & -6.446399 & 2.254980 \\ F & 6.090080 & -5.527662 & -1.932684 \\ F & 5.909722 & -7.066964 & 0.311536 \\ C & -2.724117 & 3.901389 & -0.360670 \\ C & -2.859974 & 4.620345 & 0.829825 \\ C & -3.511128 & 4.311673 & -1.441642 \\ C & -3.747189 & 5.683972 & 0.960636 \\ F & -2.131531 & 4.276932 & 1.910222 \\ C & -4.405614 & 5.376074 & -1.339039 \\ F & -3.427863 & 3.667785 & -2.610206 \\ C & -4.522438 & 6.065155 & -0.133112 \\ F & -3.866952 & 6.329408 & 2.127101 \\ F & -5.148431 & 5.742380 & -2.387895 \\ F & -5.378510 & 7.083546 & -0.023533\end{array}$

\section{Structure: ${ }^{5}$ INT1}

$\begin{array}{lrrr}\mathrm{C} & 2.581490 & -0.578766 & -0.834978 \\ \mathrm{C} & 3.883747 & 0.044627 & -0.814652 \\ \mathrm{C} & 3.683104 & 1.379930 & -0.670239 \\ \mathrm{C} & 2.255806 & 1.589674 & -0.603568 \\ \mathrm{~N} & 1.610084 & 0.382102 & -0.696855 \\ \mathrm{H} & 4.826971 & -0.477085 & -0.880162 \\ \mathrm{H} & 4.431806 & 2.153917 & -0.593553 \\ \mathrm{C} & -1.910319 & 2.727061 & -0.320600 \\ \mathrm{C} & -1.695900 & 4.154382 & -0.270887 \\ \mathrm{C} & -0.352639 & 4.355750 & -0.300454 \\ \mathrm{C} & 0.267945 & 3.053354 & -0.375255 \\ \mathrm{~N} & -0.700612 & 2.083479 & -0.381409 \\ \mathrm{H} & -2.475271 & 4.899879 & -0.212138\end{array}$




\begin{tabular}{|c|c|c|c|}
\hline $\mathrm{H}$ & 0.174641 & 5.298541 & -0.289219 \\
\hline C & -1.045033 & -2.907363 & -0.553557 \\
\hline C & -0.439413 & -4.201552 & -0.754281 \\
\hline $\mathrm{C}$ & 0.889585 & -3.992547 & -0.956230 \\
\hline C & 1.111397 & -2.570669 & -0.867725 \\
\hline $\mathrm{N}$ & -0.081480 & -1.931592 & -0.632803 \\
\hline $\mathrm{H}$ & -0.969907 & -5.142613 & -0.762728 \\
\hline $\mathrm{H}$ & 1.652151 & -4.730991 & -1.157500 \\
\hline $\mathrm{C}$ & -3.021165 & -1.445577 & -0.236799 \\
\hline $\mathrm{C}$ & -4.442023 & -1.242867 & -0.071700 \\
\hline C & -4.658101 & 0.097218 & -0.104414 \\
\hline $\mathrm{C}$ & -3.368167 & 0.730238 & -0.253839 \\
\hline $\mathrm{N}$ & -2.392323 & -0.230173 & -0.335620 \\
\hline $\mathrm{H}$ & -5.175658 & -2.026894 & 0.043827 \\
\hline $\mathrm{H}$ & -5.602640 & 0.615025 & -0.028170 \\
\hline $\mathrm{C}$ & 2.365993 & -1.956081 & -0.936972 \\
\hline $\mathrm{C}$ & -3.165414 & 2.113746 & -0.272204 \\
\hline $\mathrm{C}$ & 1.647674 & 2.839677 & -0.451980 \\
\hline $\mathrm{C}$ & -2.409226 & -2.700082 & -0.323700 \\
\hline $\mathrm{Mn}$ & -0.405811 & 0.089279 & -0.619668 \\
\hline $\mathrm{Cl}$ & -0.660126 & 0.199771 & -3.024722 \\
\hline $\mathrm{C}$ & 2.586955 & 1.235518 & 2.659126 \\
\hline $\mathrm{C}$ & 3.787757 & 1.942682 & 2.772715 \\
\hline C & 5.005190 & 1.263100 & 2.849484 \\
\hline $\mathrm{C}$ & 5.037912 & -0.132190 & 2.800812 \\
\hline $\mathrm{C}$ & 3.850375 & -0.861766 & 2.691560 \\
\hline $\mathrm{C}$ & 2.656222 & -0.150703 & 2.639818 \\
\hline $\mathrm{H}$ & 1.634874 & 1.748524 & 2.563441 \\
\hline $\mathrm{H}$ & 3.758265 & 3.025809 & 2.809138 \\
\hline $\mathrm{H}$ & 5.931817 & 1.822389 & 2.941114 \\
\hline $\mathrm{H}$ & 5.985846 & -0.661357 & 2.841617 \\
\hline $\mathrm{H}$ & 3.868505 & -1.944186 & 2.630503 \\
\hline I & 0.779435 & -1.210680 & 2.528087 \\
\hline O & -0.241728 & 0.136724 & 1.610927 \\
\hline $\mathrm{C}$ & 2.654388 & 4.725914 & 0.865296 \\
\hline $\mathrm{C}$ & 3.292223 & 4.495592 & -1.421322 \\
\hline $\mathrm{C}$ & 3.495977 & 5.824298 & 1.009964 \\
\hline $\mathrm{C}$ & 4.140536 & 5.595785 & -1.304146 \\
\hline $\mathrm{C}$ & 4.240553 & 6.262538 & -0.084050 \\
\hline $\mathrm{F}$ & 3.602348 & 6.448571 & 2.188592 \\
\hline $\mathrm{F}$ & 5.052527 & 7.314412 & 0.039162 \\
\hline $\mathrm{F}$ & 4.855055 & 6.016750 & -2.351674 \\
\hline $\mathrm{F}$ & 3.223820 & 3.871857 & -2.601002 \\
\hline $\mathrm{F}$ & 1.956916 & 4.321538 & 1.943660 \\
\hline
\end{tabular}




$\begin{array}{rrr}2.535932 & 4.030529 & -0.340801 \\ -4.364133 & 2.998754 & -0.209342 \\ -5.132411 & 3.108275 & 0.953479 \\ -4.757280 & 3.761677 & -1.313792 \\ -6.252528 & 3.933265 & 1.020440 \\ -4.801258 & 2.399033 & 2.041429 \\ -5.869198 & 4.600459 & -1.267481 \\ -4.052966 & 3.704411 & -2.448399 \\ -6.619218 & 4.683271 & -0.095706 \\ -6.971023 & 4.016171 & 2.145830 \\ -6.222487 & 5.321337 & -2.336293 \\ -7.688081 & 5.481253 & -0.041592 \\ -3.263206 & -3.910208 & -0.155800 \\ -3.136808 & -4.719530 & 0.977336 \\ -4.208916 & -4.290289 & -1.112951 \\ -3.912940 & -5.860697 & 1.159970 \\ -2.240848 & -4.403490 & 1.926298 \\ -5.003134 & -5.423861 & -0.949183 \\ -4.375192 & -3.553471 & -2.216154 \\ -4.851402 & -6.211688 & 0.190650 \\ -3.768363 & -6.615364 & 2.254879 \\ -5.902297 & -5.763619 & -1.877703 \\ -5.604428 & -7.301230 & 0.354657 \\ 3.563976 & -2.834791 & -1.047781 \\ 4.355575 & -2.862465 & -2.200458 \\ 3.957503 & -3.653897 & 0.013981 \\ 5.491070 & -3.666541 & -2.291794 \\ 4.037076 & -2.094628 & -3.245619 \\ 5.084658 & -4.466556 & -0.051278 \\ 3.239628 & -3.665663 & 1.156343 \\ 5.854067 & -4.472380 & -1.213873 \\ 6.230629 & -3.673154 & -3.404075 \\ -538829 & -5.245497 & -1.291780\end{array}$

\section{Structure: ${ }^{3} \mathrm{TS} 1$}

$\mathrm{C}$
$\mathrm{C}$
$\mathrm{C}$
$\mathrm{C}$
$\mathrm{N}$
$\mathrm{H}$
$\mathrm{H}$

$$
\begin{aligned}
& -2.440334 \\
& -3.840282 \\
& -3.907883 \\
& -2.549623 \\
& -1.674795 \\
& -4.662493 \\
& -4.793791
\end{aligned}
$$$$
\begin{array}{rr}
1.323261 & -0.624277 \\
0.969645 & -0.666951 \\
-0.385104 & -0.715780 \\
-0.873177 & -0.708816 \\
0.182465 & -0.633203 \\
1.668826 & -0.639368 \\
-1.002129 & -0.740660
\end{array}
$$ 


\begin{tabular}{|c|c|c|c|}
\hline $\mathrm{C}$ & 1.311561 & -2.792466 & -0.565431 \\
\hline C & 0.839153 & -4.145071 & -0.739440 \\
\hline C & -0.514614 & -4.084622 & -0.841166 \\
\hline C & -0.882487 & -2.694904 & -0.734349 \\
\hline $\mathrm{N}$ & 0.243032 & -1.922777 & -0.566132 \\
\hline $\mathrm{H}$ & 1.467628 & -5.021886 & -0.795779 \\
\hline $\mathrm{H}$ & -1.204255 & -4.902395 & -0.992837 \\
\hline C & 1.568143 & 2.887911 & -0.306767 \\
\hline $\mathrm{C}$ & 1.215135 & 4.291725 & -0.307747 \\
\hline C & -0.135138 & 4.359235 & -0.418194 \\
\hline C & -0.619589 & 2.996509 & -0.477131 \\
\hline $\mathrm{N}$ & 0.433182 & 2.126650 & -0.412966 \\
\hline $\mathrm{H}$ & 1.914228 & 5.111776 & -0.234924 \\
\hline $\mathrm{H}$ & -0.751472 & 5.245092 & -0.465065 \\
\hline C & 3.230688 & 1.053997 & -0.227523 \\
\hline C & 4.589781 & 0.569504 & -0.122921 \\
\hline C & 4.531024 & -0.785607 & -0.169452 \\
\hline C & 3.136623 & -1.138628 & -0.319651 \\
\hline $\mathrm{N}$ & 2.370720 & -0.005405 & -0.347417 \\
\hline $\mathrm{H}$ & 5.468947 & 1.190533 & -0.034276 \\
\hline $\mathrm{H}$ & 5.351232 & -1.485985 & -0.110828 \\
\hline C & -1.968126 & 2.637500 & -0.558510 \\
\hline C & 2.657734 & -2.448161 & -0.419271 \\
\hline C & -2.196719 & -2.223501 & -0.762361 \\
\hline C & 2.876033 & 2.406507 & -0.212238 \\
\hline Mn & 0.346520 & 0.093503 & -0.493480 \\
\hline $\mathrm{Cl}$ & 0.558369 & 0.224362 & -2.780718 \\
\hline $\mathrm{C}$ & -2.510165 & 1.121140 & 2.677592 \\
\hline C & -3.769248 & 1.715435 & 2.805128 \\
\hline C & -4.916473 & 0.929051 & 2.925660 \\
\hline $\mathrm{C}$ & -4.820804 & -0.463329 & 2.900430 \\
\hline $\mathrm{C}$ & -3.574949 & -1.082485 & 2.769252 \\
\hline C & -2.449598 & -0.267456 & 2.678627 \\
\hline $\mathrm{H}$ & -1.616665 & 1.722993 & 2.552449 \\
\hline $\mathrm{H}$ & -3.837991 & 2.797005 & 2.811372 \\
\hline $\mathrm{H}$ & -5.888047 & 1.403121 & 3.030816 \\
\hline $\mathrm{H}$ & -5.713678 & -1.077618 & 2.974247 \\
\hline $\mathrm{H}$ & -3.495396 & -2.162270 & 2.725048 \\
\hline I & -0.516180 & -1.198966 & 2.555928 \\
\hline O & 0.335068 & 0.256943 & 1.321432 \\
\hline C & -3.295546 & -3.228429 & -0.812630 \\
\hline C & -3.561602 & -4.058579 & 0.279882 \\
\hline C & -4.112778 & -3.377590 & -1.937709 \\
\hline C & -4.591435 & -4.994049 & 0.270775 \\
\hline
\end{tabular}




\section{Structure:}

\begin{tabular}{|c|c|c|}
\hline-5.152505 & -4.305775 & -1.972974 \\
\hline-5.389781 & -5.117952 & -0.865281 \\
\hline-4.821849 & -5.765166 & 1.339600 \\
\hline 2.813175 & -3.958647 & 1.397306 \\
\hline 3.9 & -2.609189 & -3.012685 \\
\hline 5.91 & -4.425675 & -3.060 \\
\hline 6.38 & -6.00979 & -0.889 \\
\hline & 3.406 & -0.084 \\
\hline 4.68 & 3.559988 & 1.1 \\
\hline 4.34 & & $-1 \cdot 1$ \\
\hline & $4.4 \varepsilon$ & 1.2 \\
\hline 4.37 & 2.79 & \\
\hline 5.37 & 5.16 & $-1 \cdot 0$ \\
\hline 3.7 & 4.12 & $-2 \cdot 3$ \\
\hline & $5.2 \varepsilon$ & \\
\hline & 6272 & \\
\hline 5.7 & 5.93 & -2.0 \\
\hline 7.0 & 6.17 & 0.2 \\
\hline 3.6 & -3.56 & -0 \\
\hline & -4.47 & \\
\hline 4.56 & $-3.7 c$ & $-1 \cdot 3$ \\
\hline 4.6 & -5.46 & \\
\hline & -4 & \\
\hline 5.49 & -4.83 & $-1 \cdot 3$ \\
\hline 4.57 & -3.01 & -2.4 \\
\hline & -5.6 & -0 \\
\hline 4.6 & -6.26 & 1.9 \\
\hline 6.35 & -5.04 & $-2 \cdot 3$ \\
\hline 6.38 & -6.674001 & -0.1 \\
\hline-2.97 & 3.735366 & -0.5 \\
\hline-3.16 & 4.521240 & 0.5 \\
\hline-3.77 & 4.024025 & -1.65 \\
\hline-4.11 & 5.539233 & \\
\hline-2.43 & 4.293504 & 1.7 \\
\hline-4.72841 & 5.038222 & -1.63 \\
\hline-3.63716 & 3.308246 & -2.7 \\
\hline-4.89902 & 5.799588 & -0.47 \\
\hline-4.28573 & 6.258159 & 1.763 \\
\hline & & -2.7 \\
\hline .81 & 6.772160 & -0.4 \\
\hline
\end{tabular}




\begin{tabular}{|c|c|c|c|}
\hline $\mathrm{C}$ & 3.818633 & 0.629914 & -0.859705 \\
\hline C & 3.445781 & 1.905814 & -0.583784 \\
\hline C & 2.003996 & 1.915252 & -0.497243 \\
\hline $\mathrm{N}$ & 1.524871 & 0.647875 & -0.700941 \\
\hline $\mathrm{H}$ & 4.821213 & 0.246938 & -0.979374 \\
\hline $\mathrm{H}$ & 4.086008 & 2.762562 & -0.438143 \\
\hline C & -2.283610 & 2.450898 & -0.365687 \\
\hline C & -2.268980 & 3.889538 & -0.258104 \\
\hline $\mathrm{C}$ & -0.967239 & 4.270407 & -0.164850 \\
\hline $\mathrm{C}$ & -0.168478 & 3.071234 & -0.239221 \\
\hline $\mathrm{N}$ & -0.993913 & 1.979740 & -0.349795 \\
\hline $\mathrm{H}$ & -3.145808 & 4.520202 & -0.241826 \\
\hline $\mathrm{H}$ & -0.576114 & 5.273902 & -0.080728 \\
\hline C & -0.619293 & -2.982771 & -0.561190 \\
\hline C & 0.155973 & -4.179674 & -0.793102 \\
\hline C & 1.421452 & -3.780278 & -1.090250 \\
\hline C & 1.438580 & -2.338744 & -1.007495 \\
\hline $\mathrm{N}$ & 0.181707 & -1.883338 & -0.707821 \\
\hline $\mathrm{H}$ & -0.231662 & -5.187756 & -0.764878 \\
\hline $\mathrm{H}$ & 2.268933 & -4.401193 & -1.342081 \\
\hline $\mathrm{C}$ & -2.782821 & -1.820648 & -0.241148 \\
\hline $\mathrm{C}$ & -4.222338 & -1.824033 & -0.114332 \\
\hline $\mathrm{C}$ & -4.635633 & -0.536283 & -0.236647 \\
\hline C & -3.449950 & 0.276316 & -0.381112 \\
\hline $\mathrm{N}$ & -2.342430 & -0.529751 & -0.396090 \\
\hline $\mathrm{H}$ & -4.834826 & -2.702300 & 0.024838 \\
\hline $\mathrm{H}$ & -5.648729 & -0.163173 & -0.214369 \\
\hline C & 2.587259 & -1.546880 & -1.105564 \\
\hline $\mathrm{C}$ & -3.443652 & 1.673874 & -0.412328 \\
\hline $\mathrm{C}$ & 1.228585 & 3.055053 & -0.262273 \\
\hline $\mathrm{C}$ & -1.989166 & -2.970358 & -0.273064 \\
\hline $\mathrm{Mn}$ & -0.418867 & 0.069679 & -0.560277 \\
\hline $\mathrm{Cl}$ & -0.615813 & 0.150006 & -2.947797 \\
\hline $\mathrm{C}$ & 2.316537 & 1.382658 & 2.785969 \\
\hline $\mathrm{C}$ & 3.404626 & 2.251396 & 2.907821 \\
\hline C & 4.711479 & 1.761813 & 2.865155 \\
\hline C & 4.943662 & 0.397169 & 2.684339 \\
\hline C & 3.871868 & -0.492026 & 2.567462 \\
\hline C & 2.580431 & 0.024446 & 2.639335 \\
\hline $\mathrm{H}$ & 1.299146 & 1.757256 & 2.780264 \\
\hline $\mathrm{H}$ & 3.216534 & 3.310501 & 3.043334 \\
\hline $\mathrm{H}$ & 5.549567 & 2.445565 & 2.965508 \\
\hline $\mathrm{H}$ & 5.959250 & 0.015015 & 2.630669 \\
\hline $\mathrm{H}$ & 4.046633 & -1.549593 & 2.409843 \\
\hline
\end{tabular}


0.907646

$-0.255894$

1. 909401

2. 653820

2.579620

3. 331113

3. 291819

2. 554292

3. 940537

4.013426

2. 714640

1.239741

1.935654

$-4.751816$

$-5.614958$

$-5.154193$

$-6.835891$

$-5.277600$

$-6.367216$

$-4.361153$

$-7.210307$

$-7.644301$

$-6.728534$

$-8.376088$

$-2.626691$

$-2.304965$

$-3.537439$

$-2.862098$

$-1.430522$

$-4.115109$

$-3.881672$

$-3.772920$

$-2.535464$

$-4.986387$

$-4.317254$

3. 892320

4.613945

4.462815

5.849481

4.126600

5.693038

3. 821741

6.388612

6.518083
$-1.317745$

$-0.033755$

4.964105

4.990339

6.156747

6.187431

6.772533

6.703690

7.915974

6.778662

4.450889

4.391432

4.345885

2. 387998

2.403684

3.086711

3.073099

1.754820

3.770544

3.117216

3.760542

3.067559

4.431038

4.409499

$-4.284118$

$-4.965725$

$-4.895884$

$-6.202014$

$-4.426237$

$-6.129452$

$-4.292159$

$-6.784308$

$-6.829136$

$-6.691166$

$-7.967816$

$-2.232241$

$-2.065190$

$-3.047461$

$-2.679048$

$-1.290145$

$-3.673275$

$-3.243013$

$-3.487212$

$-2.501349$
2.557279

1.358075

1.219141

$-1.046308$

1.473038

$-0.819428$

0.445216

2. 693643

0.674180

$-1.804472$

$-2.267928$

2. 236082

$-0.033786$

$-0.448674$

0.650851

$-1.591971$

0.618183

1.774224

$-1.644870$

$-2.667535$

$-0.535067$

1.683982

$-2.749356$

$-0.575908$

0.023096

1. 201473

$-0.842680$

1.515205

2.067190

$-0.547243$

$-1.986050$

0.634690

2.650306

$-1.390838$

0.924551

$-1.325221$

$-2.511961$

$-0.343321$

$-2.712905$

$-3.484323$

$-0.519825$

0.825660

$-1.713416$

$-3.855657$ 
6.210929

7.570245
$-4.441518$

$-4.079613$
0.445348

$-1.896504$

\section{Structure: ${ }^{3}$ INT2}

C

C

C

C

$\mathrm{N}$

$\mathrm{H}$

$\mathrm{H}$

C

C

C

C

$\mathrm{N}$

$\mathrm{H}$

$\mathrm{H}$

C

C

C

C

$\mathrm{N}$

$\mathrm{H}$

$\mathrm{H}$

C

C

C

C

N

$\mathrm{H}$

$\mathrm{H}$

C

C

C

$\mathrm{C}$

$\mathrm{Mn}$

$\mathrm{Cl}$

$\mathrm{O}$

C

C

C

C

$\begin{array}{rrr}0.995013 & -2.897333 & -0.136459 \\ 0.538401 & -4.265500 & -0.256968 \\ -0.817354 & -4.220245 & -0.296823 \\ -1.187756 & -2.825271 & -0.193389 \\ -0.067587 & -2.041923 & -0.127642 \\ 1.172758 & -5.137910 & -0.309908 \\ -1.503991 & -5.048898 & -0.386635 \\ -2.796237 & 1.189101 & 0.062985 \\ -4.178645 & 0.814392 & 0.248035 \\ -4.225069 & -0.542168 & 0.208306 \\ -2.869744 & -0.999209 & 0.005815 \\ -2.017243 & 0.067307 & -0.055971 \\ -4.999287 & 1.499681 & 0.399784 \\ -5.090615 & -1.177849 & 0.322337 \\ 2.869750 & 0.999204 & 0.005805 \\ 4.225076 & 0.542163 & 0.208281 \\ 4.178654 & -0.814398 & 0.248008 \\ 2.796243 & -1.189106 & 0.062984 \\ 2.017247 & -0.067313 & -0.055962 \\ 5.090625 & 1.177843 & 0.322299 \\ 4.999299 & -1.499686 & 0.399745 \\ 1.187762 & 2.825267 & -0.193398 \\ 0.817359 & 4.220241 & -0.296822 \\ -0.538396 & 4.265494 & -0.256967 \\ -0.995006 & 2.897326 & -0.136469 \\ 0.067592 & 2.041917 & -0.127656 \\ -1.503996 & 5.048895 & -0.386625 \\ -1.172754 & 5.137904 & -0.309901 \\ -2.339986 & -2.512187 & -0.014405 \\ -2.339980 & 2.512181 & -0.014411 \\ -2.502928 & -2.351161 & -0.114286 \\ -0.5000003901 & -0.000019 & -1.836547 \\ 0.000005 & -0.000004 & -0.238180 \\ -5.254521 & 4.778933 & -0.934937\end{array}$




\begin{tabular}{|c|c|c|c|}
\hline C & -4.362618 & 5.501277 & 1.190398 \\
\hline C & -5.284774 & 5.647804 & 0.155036 \\
\hline C & -3.603708 & -3.355117 & -0.135283 \\
\hline C & -4.509002 & -3.408810 & -1.200738 \\
\hline C & -3.778537 & -4.270538 & 0.908156 \\
\hline $\mathrm{C}$ & -5.547481 & -4.335928 & -1.234086 \\
\hline C & -4.809947 & -5.206693 & 0.894778 \\
\hline C & -5.696333 & -5.237398 & -0.180916 \\
\hline $\mathrm{C}$ & 3.360328 & -3.596376 & 0.044734 \\
\hline C & 4.297913 & -3.767869 & -0.979308 \\
\hline C & 3.414811 & -4.482633 & 1.126212 \\
\hline C & 5.254515 & -4.778947 & -0.934949 \\
\hline C & 4.362641 & -5.501270 & 1.190405 \\
\hline C & 5.284783 & -5.647809 & 0.155032 \\
\hline C & 3.603709 & 3.355118 & -0.135287 \\
\hline C & 4.508991 & 3.408836 & -1.200750 \\
\hline C & 3.778538 & 4.270525 & 0.908164 \\
\hline $\mathrm{C}$ & 5.547464 & 4.335962 & -1.234092 \\
\hline $\mathrm{C}$ & 4.809942 & 5.206687 & 0.894792 \\
\hline $\mathrm{C}$ & 5.696319 & 5.237415 & -0.180908 \\
\hline $\mathrm{F}$ & -2.943985 & -4.259592 & 1.952833 \\
\hline $\mathrm{F}$ & -4.387040 & -2.553796 & -2.222658 \\
\hline $\mathrm{F}$ & -6.396009 & -4.369035 & -2.265428 \\
\hline $\mathrm{F}$ & -6.686918 & -6.129484 & -0.202610 \\
\hline $\mathrm{F}$ & -4.957681 & -6.068307 & 1.904793 \\
\hline $\mathrm{F}$ & 2.943993 & 4.259559 & 1.952846 \\
\hline $\mathrm{F}$ & 4.957678 & 6.068288 & 1.904818 \\
\hline $\mathrm{F}$ & 6.686898 & 6.129509 & -0.202597 \\
\hline $\mathrm{F}$ & 6.395981 & 4.369092 & -2.265442 \\
\hline $\mathrm{F}$ & 4.387024 & 2.553839 & -2.222684 \\
\hline $\mathrm{F}$ & -2.542000 & 4.362567 & 2.131613 \\
\hline $\mathrm{F}$ & -4.288080 & 2.948034 & -2.036992 \\
\hline F & -6.136313 & 4.923839 & -1.928120 \\
\hline $\mathrm{F}$ & -6.196032 & 6.619672 & 0.206702 \\
\hline $\mathrm{F}$ & -4.396002 & 6.332867 & 2.235170 \\
\hline $\mathrm{F}$ & 2.542037 & -4.362550 & 2.131635 \\
\hline F & 4.396040 & -6.332850 & 2.235185 \\
\hline F & 6.196042 & -6.619676 & 0.206695 \\
\hline F & 6.136295 & -4.923862 & -1.928142 \\
\hline$F$ & 4.288061 & -2.948057 & -2.037008 \\
\hline
\end{tabular}

\section{Structure: ${ }^{5}$ INT2}




\begin{tabular}{|c|c|c|c|}
\hline $\mathrm{C}$ & 4.239404 & 0.678301 & -0.202186 \\
\hline C & 4.239459 & -0.678139 & -0.202405 \\
\hline C & 2.857324 & -1.095254 & -0.137563 \\
\hline $\mathrm{N}$ & 2.036727 & 0.000092 & -0.105506 \\
\hline $\mathrm{H}$ & 5.091431 & 1.339404 & -0.252823 \\
\hline $\mathrm{H}$ & 5.091564 & -1.339139 & -0.253223 \\
\hline C & -1.090767 & -2.854783 & -0.035422 \\
\hline C & -0.672531 & -4.238057 & -0.023083 \\
\hline $\mathrm{C}$ & 0.683618 & -4.237187 & -0.044299 \\
\hline C & 1.099324 & -2.853710 & -0.086341 \\
\hline $\mathrm{N}$ & 0.003840 & -2.033273 & -0.078202 \\
\hline $\mathrm{H}$ & -1.332390 & -5.092123 & 0.000552 \\
\hline $\mathrm{H}$ & 1.345656 & -5.089752 & -0.024061 \\
\hline C & -1.090749 & 2.854731 & -0.035558 \\
\hline C & -0.672629 & 4.238076 & -0.023223 \\
\hline C & 0.683519 & 4.237300 & -0.044083 \\
\hline $\mathrm{C}$ & 1.099356 & 2.853890 & -0.086045 \\
\hline $\mathrm{N}$ & 0.003696 & 2.033340 & -0.078185 \\
\hline $\mathrm{H}$ & -1.332553 & 5.092088 & 0.000140 \\
\hline $\mathrm{H}$ & 1.345498 & 5.089917 & -0.023784 \\
\hline C & -2.847134 & 1.095218 & 0.016635 \\
\hline $\mathrm{C}$ & -4.225463 & 0.678207 & 0.137684 \\
\hline $\mathrm{C}$ & -4.225395 & -0.678250 & 0.137932 \\
\hline C & -2.847005 & -1.095160 & 0.016966 \\
\hline $\mathrm{N}$ & -2.028892 & -0.000065 & -0.050576 \\
\hline $\mathrm{H}$ & -5.073354 & 1.339867 & 0.232560 \\
\hline $\mathrm{H}$ & -5.073207 & -1.339971 & 0.233028 \\
\hline C & 2.434809 & 2.431388 & -0.124188 \\
\hline $\mathrm{C}$ & -2.425779 & -2.431643 & 0.003823 \\
\hline $\mathrm{C}$ & 2.434968 & -2.431168 & -0.124784 \\
\hline $\mathrm{C}$ & -2.425963 & 2.431515 & 0.003403 \\
\hline $\mathrm{Mn}$ & 0.002549 & 0.000023 & -0.190731 \\
\hline $\mathrm{Cl}$ & 0.001556 & 0.000131 & 2.258946 \\
\hline O & -0.005622 & 0.000007 & -1.845440 \\
\hline $\mathrm{C}$ & -3.482529 & 3.481706 & 0.051724 \\
\hline C & -4.358397 & 3.673450 & -1.021994 \\
\hline C & -3.641327 & 4.304469 & 1.171770 \\
\hline C & -5.354260 & 4.646455 & -0.989439 \\
\hline C & -4.629376 & 5.284874 & 1.224664 \\
\hline $\mathrm{C}$ & -5.487755 & 5.454241 & 0.139103 \\
\hline C & -3.482428 & -3.481738 & 0.052506 \\
\hline C & -4.358630 & -3.673520 & -1.020933 \\
\hline C & -3.641103 & -4.304231 & 1.172763 \\
\hline $\mathrm{C}$ & -5.354788 & -4.646194 & -0.98786 \\
\hline
\end{tabular}




\begin{tabular}{|c|c|c|c|}
\hline C & -4.629441 & -5.284328 & 1.226162 \\
\hline C & -5.488242 & -5.453638 & 0.140933 \\
\hline $\mathrm{C}$ & 3.488501 & -3.485301 & -0.146897 \\
\hline $\mathrm{C}$ & 3.652423 & -4.322783 & -1.255093 \\
\hline $\mathrm{C}$ & 4.348923 & -3.673547 & 0.939955 \\
\hline $\mathrm{C}$ & 4.631976 & -5.312239 & -1.286404 \\
\hline $\mathrm{C}$ & 5.336460 & -4.655841 & 0.929033 \\
\hline $\mathrm{C}$ & 5.476022 & -5.477149 & -0.188949 \\
\hline $\mathrm{C}$ & 3.488512 & 3.485369 & -0.145837 \\
\hline $\mathrm{C}$ & 3.653016 & 4.322840 & -1.253954 \\
\hline C & 4.348716 & 3.673267 & 0.941252 \\
\hline $\mathrm{C}$ & 4.632988 & 5.311901 & -1.284979 \\
\hline $\mathrm{C}$ & 5.336652 & 4.655153 & 0.930612 \\
\hline $\mathrm{C}$ & 5.476872 & 5.476393 & -0.187345 \\
\hline $\mathrm{F}$ & 4.234757 & 2.896014 & 2.023019 \\
\hline F & 6.144937 & 4.816459 & 1.981509 \\
\hline $\mathrm{F}$ & 6.418873 & 6.419572 & -0.207006 \\
\hline $\mathrm{F}$ & -2.829677 & 4.161131 & 2.224679 \\
\hline $\mathrm{F}$ & -2.829082 & -4.160936 & 2.225388 \\
\hline $\mathrm{F}$ & -4.761911 & -6.056787 & 2.307761 \\
\hline $\mathrm{F}$ & -6.437847 & -6.388471 & 0.182355 \\
\hline $\mathrm{F}$ & 4.235557 & -2.896280 & 2.021778 \\
\hline $\mathrm{F}$ & 6.144948 & -4.817487 & 1.979721 \\
\hline $\mathrm{F}$ & 6.417625 & -6.420719 & -0.208860 \\
\hline F & 4.769825 & -6.098038 & -2.357882 \\
\hline F & 2.853090 & -4.184083 & -2.319123 \\
\hline $\mathrm{F}$ & 2.853878 & 4.184530 & -2.318180 \\
\hline F & 4.771395 & 6.097684 & -2.356393 \\
\hline F & -4.251499 & -2.908171 & -2.112891 \\
\hline $\mathrm{F}$ & -6.177491 & -4.811566 & -2.02711 \\
\hline $\mathrm{F}$ & -4.251213 & 2.907798 & -2.113731 \\
\hline $\mathrm{F}$ & -6.176610 & 4.811837 & -2.028965 \\
\hline F & -6.437076 & 6.389387 & 0.180018 \\
\hline$F$ & -4.761985 & 6.057591 & 2.30606 \\
\hline
\end{tabular}

\section{Structure: ${ }^{3} \mathrm{TS} 2 \mathrm{a}$}

C

$\mathrm{C}$

C

C

$\mathrm{N}$

$\mathrm{H}$

$\mathrm{H}$
$1.243979-2.485903-1.556125$

$2.354669-3.399128-1.458255$

$3.408998-2.697805-0.967055$

$2.972776-1.332548-0.801719$

$1.645464-1.233931-1.154508$

$2.323093-4.446597-1.718860$

$\begin{array}{llll}4.393413 & -3.070419 & -0.731219\end{array}$ 


\begin{tabular}{|c|c|c|c|}
\hline C & 2.218872 & 2.897207 & -0.655744 \\
\hline C & 3.567187 & 3.302541 & -0.331159 \\
\hline C & 4.290249 & 2.168801 & -0.144714 \\
\hline $\mathrm{C}$ & 3.399442 & 1.057009 & -0.381160 \\
\hline $\mathrm{N}$ & 2.146343 & 1.528376 & -0.677640 \\
\hline $\mathrm{H}$ & 3.902950 & 4.325239 & -0.241993 \\
\hline $\mathrm{H}$ & 5.337928 & 2.082039 & 0.102603 \\
\hline $\mathrm{C}$ & -2.429792 & -0.232752 & -1.558042 \\
\hline $\mathrm{C}$ & -3.301066 & -1.325717 & -1.918995 \\
\hline $\mathrm{C}$ & -2.512353 & -2.408543 & -2.151389 \\
\hline C & -1.149622 & -1.997879 & -1.913721 \\
\hline $\mathrm{N}$ & -1.128200 & -0.669808 & -1.573354 \\
\hline $\mathrm{H}$ & -4.375209 & -1.266971 & -2.004229 \\
\hline $\mathrm{H}$ & -2.821374 & -3.402603 & -2.439369 \\
\hline C & -1.990789 & 2.151768 & -1.100565 \\
\hline $\mathrm{C}$ & -2.420934 & 3.530347 & -1.062318 \\
\hline $\mathrm{C}$ & -1.299303 & 4.295688 & -1.036434 \\
\hline $\mathrm{C}$ & -0.171613 & 3.393311 & -1.004212 \\
\hline $\mathrm{N}$ & -0.624005 & 2.101009 & -1.065536 \\
\hline $\mathrm{H}$ & -3.448979 & 3.860544 & -1.096991 \\
\hline $\mathrm{C}$ & -0.050550 & -2.859394 & -1.922381 \\
\hline $\mathrm{C}$ & 1.158738 & 3.787072 & -0.848101 \\
\hline $\mathrm{C}$ & 3.798853 & -0.281997 & -0.387609 \\
\hline $\mathrm{C}$ & -2.854848 & 1.066577 & -1.265783 \\
\hline $\mathrm{Mn}$ & 0.494097 & 0.415215 & -1.070216 \\
\hline $\mathrm{Cl}$ & 0.888449 & 0.788474 & -3.347906 \\
\hline $\mathrm{C}$ & -4.312113 & 1.321747 & -1.094645 \\
\hline $\mathrm{C}$ & -4.810595 & 1.670190 & 0.167288 \\
\hline $\mathrm{C}$ & -5.232813 & 1.220609 & -2.141736 \\
\hline $\mathrm{C}$ & -6.166568 & 1.894475 & 0.381833 \\
\hline $\mathrm{C}$ & -6.597347 & 1.426562 & -1.945804 \\
\hline $\mathrm{C}$ & -7.060977 & 1.766145 & -0.676717 \\
\hline $\mathrm{C}$ & -0.298267 & -4.291941 & -2.249725 \\
\hline C & -0.258442 & -5.275149 & -1.256660 \\
\hline $\mathrm{C}$ & -0.597782 & -4.704691 & -3.551196 \\
\hline $\mathrm{C}$ & -0.494616 & -6.617768 & -1.537621 \\
\hline C & -0.844511 & -6.042165 & -3.856901 \\
\hline $\mathrm{C}$ & -0.789559 & -7.000145 & -2.845764 \\
\hline $\mathrm{C}$ & 5.193232 & -0.601996 & 0.033431 \\
\hline C & 6.131879 & -1.133992 & -0.857933 \\
\hline $\mathrm{C}$ & 5.617414 & -0.396189 & 1.350825 \\
\hline $\mathrm{C}$ & 7.416819 & -1.486102 & -0.453155 \\
\hline $\mathrm{C}$ & 6.894205 & -0.743264 & 1.780560 \\
\hline $\mathrm{C}$ & 7.797765 & -1.291286 & 0.873443 \\
\hline
\end{tabular}




\begin{tabular}{|c|c|c|c|}
\hline C & 1.467585 & 5.245112 & -0.830513 \\
\hline C & 2.194214 & 5.835263 & -1.870207 \\
\hline C & 1.061738 & 6.072971 & 0.220584 \\
\hline C & 2.503306 & 7.193904 & -1.870316 \\
\hline C & 1.351779 & 7.435135 & 0.237769 \\
\hline & 2.077657 & 7.995244 & -0.812174 \\
\hline $\mathrm{F}$ & -3.981606 & 1.782453 & 1.209362 \\
\hline $\mathrm{F}$ & -6.620993 & 2.206436 & 1.602444 \\
\hline $\mathrm{F}$ & -8.369740 & 1.954167 & -0.468125 \\
\hline $\mathrm{F}$ & -7.457546 & 1.308559 & -2.962035 \\
\hline $\mathrm{F}$ & -4.813357 & 0.895779 & -3.371562 \\
\hline $\mathrm{F}$ & 2.617878 & 5.087748 & -2.894452 \\
\hline $\mathrm{F}$ & 3.199647 & 7.732646 & -2.875836 \\
\hline $\mathrm{F}$ & 2.365250 & 9.298354 & -0.803562 \\
\hline $\mathrm{F}$ & 0.947233 & 8.203963 & 1.254393 \\
\hline F & 5.797896 & -1.334003 & -2.140326 \\
\hline $\mathrm{F}$ & 8.287111 & -2.000715 & -1.328523 \\
\hline $\mathrm{F}$ & 9.027732 & -1.623647 & 1.270675 \\
\hline $\mathrm{F}$ & 7.258041 & -0.543390 & 3.050420 \\
\hline $\mathrm{F}$ & 4.792925 & 0.166109 & 2.244431 \\
\hline $\mathrm{F}$ & 0.015737 & -4.931958 & 0.013772 \\
\hline $\mathrm{F}$ & -0.448321 & -7.536182 & -0.567358 \\
\hline $\mathrm{F}$ & -1.021422 & -8.283287 & -3.127972 \\
\hline $\mathrm{F}$ & -1.128895 & -6.411639 & -5.108918 \\
\hline F & -0.662396 & -3.804223 & -4.536834 \\
\hline C & -5.103763 & -0.929058 & 2.511853 \\
\hline C & -4.534927 & -0.816678 & 3.763104 \\
\hline C & -3.155571 & -1.115376 & 3.929323 \\
\hline C & -2.349521 & -1.511474 & 2.814795 \\
\hline $\mathrm{C}$ & -2.984552 & -1.602297 & 1.548898 \\
\hline $\mathrm{C}$ & -4.325077 & -1.333470 & 1.404429 \\
\hline $\mathrm{H}$ & -5.122461 & -0.506762 & 4.614190 \\
\hline $\mathrm{C}$ & -2.554338 & -1.025897 & 5.212315 \\
\hline C & -0.954360 & -1.804249 & 3.005563 \\
\hline $\mathrm{H}$ & -2.418072 & -1.891635 & 0.674158 \\
\hline $\mathrm{H}$ & -4.808353 & -1.419712 & 0.437900 \\
\hline $\mathrm{C}$ & -0.409583 & -1.718541 & 4.302045 \\
\hline $\mathrm{C}$ & -1.221982 & -1.331528 & 5.388194 \\
\hline O & -6.432506 & -0.694910 & 2.171731 \\
\hline C & -8.712861 & -0.148865 & 2.292992 \\
\hline $\mathrm{H}$ & -9.506465 & 0.080468 & 3.004851 \\
\hline $\mathrm{H}$ & -8.652720 & 0.645833 & 1.544482 \\
\hline $\mathrm{H}$ & -8.931962 & -1.084787 & 1.769902 \\
\hline $\mathrm{C}$ & -7.403053 & -0.255853 & 3.033968 \\
\hline
\end{tabular}




$\begin{array}{lrrr}\mathrm{O} & -7.230584 & -0.000347 & 4.200421 \\ \mathrm{C} & 1.030374 & -2.119889 & 4.509905 \\ \mathrm{C} & 1.893462 & -1.761267 & 3.286159 \\ \mathrm{C} & 1.854111 & -1.618486 & 5.714165 \\ \mathrm{H} & 1.554985 & -2.085183 & 6.657601 \\ \mathrm{C} & 3.302811 & -1.993101 & 5.305529 \\ \mathrm{H} & 3.611147 & -2.956800 & 5.728910 \\ \mathrm{C} & 3.285830 & -2.142731 & 3.770134 \\ \mathrm{O} & 4.217716 & -2.505382 & 3.082230 \\ \mathrm{C} & 1.935514 & -0.229666 & 3.030219 \\ \mathrm{H} & 2.399385 & -0.027859 & 2.066502 \\ \mathrm{H} & 0.932125 & 0.198642 & 3.009400 \\ \mathrm{H} & 2.516089 & 0.294990 & 3.796072 \\ \mathrm{H} & 4.059584 & -1.261348 & 5.606695 \\ \mathrm{H} & 1.742458 & -0.535660 & 5.834017 \\ \mathrm{C} & 1.349541 & -2.528723 & 2.089749 \\ \mathrm{C} & -0.098021 & -2.107871 & 1.822001 \\ \mathrm{H} & 1.386836 & -3.607790 & 2.296493 \\ \mathrm{H} & 1.960579 & -2.363630 & 1.204680 \\ \mathrm{H} & -0.575465 & -2.784270 & 1.107775 \\ \mathrm{H} & -0.044472 & -1.104355 & 1.221981 \\ \mathrm{H} & -0.782077 & -1.272946 & 6.379537 \\ \mathrm{H} & -3.163947 & -0.716943 & 6.057549 \\ \mathrm{H} & -1.234313 & 5.373465 & -1.030226 \\ \mathrm{~F} & 0.366810 & 5.559722 & 1.245022 \\ \mathrm{O} & 0.135815 & 0.276156 & 0.596263 \\ \mathrm{H} & 1.035075 & -3.222902 & 4.577193\end{array}$

\section{Structure: ${ }^{5}$ TS2a}

$\begin{array}{lrrr}\mathrm{C} & 1.708277 & 1.704469 & 1.208850 \\ \mathrm{C} & 3.000661 & 1.348282 & 1.739255 \\ \mathrm{C} & 2.941748 & 0.033177 & 2.078253 \\ \mathrm{C} & 1.633061 & -0.444196 & 1.707386 \\ \mathrm{~N} & 0.898137 & 0.599163 & 1.194172 \\ \mathrm{H} & 3.831573 & 2.026156 & 1.865747 \\ \mathrm{H} & 3.718498 & -0.565130 & 2.529446 \\ \mathrm{C} & -2.020525 & -2.297912 & 0.395321 \\ \mathrm{C} & -1.621636 & -3.661350 & 0.638997 \\ \mathrm{C} & -0.385287 & -3.623769 & 1.200312 \\ \mathrm{C} & -0.011583 & -2.233441 & 1.304616 \\ \mathrm{~N} & -1.022131 & -1.450974 & 0.803312 \\ \mathrm{H} & -2.215721 & -4.533692 & 0.411654 \\ \mathrm{H} & 0.237133 & -4.465815 & 1.459810\end{array}$




\begin{tabular}{|c|c|c|c|}
\hline $\mathrm{C}$ & -2.158502 & 3.380802 & 0.322754 \\
\hline C & -1.737620 & 4.762548 & 0.298858 \\
\hline C & -0.383994 & 4.768165 & 0.409206 \\
\hline C & 0.031841 & 3.394020 & 0.558608 \\
\hline $\mathrm{N}$ & -1.065802 & 2.572111 & 0.498670 \\
\hline $\mathrm{H}$ & -2.396838 & 5.611622 & 0.196293 \\
\hline $\mathrm{H}$ & 0.279430 & 5.620797 & 0.428758 \\
\hline C & -3.809869 & 1.599090 & -0.054027 \\
\hline $\mathrm{C}$ & -5.102510 & 1.140780 & -0.49693 \\
\hline $\mathrm{C}$ & -5.023749 & -0.208985 & -0.636749 \\
\hline C & -3.695452 & -0.600397 & -0.239016 \\
\hline $\mathrm{N}$ & -2.970020 & 0.521665 & 0.093445 \\
\hline $\mathrm{H}$ & -5.948545 & 1.779891 & -0.702964 \\
\hline $\mathrm{H}$ & -5.797853 & -0.884823 & -0.968666 \\
\hline C & 1.337987 & 3.002110 & 0.840849 \\
\hline C & -3.258927 & -1.919869 & -0.130942 \\
\hline C & 1.222112 & -1.778159 & 1.780297 \\
\hline C & -3.465316 & 2.942790 & 0.102117 \\
\hline $\mathrm{Mn}$ & -1.047982 & 0.557803 & 0.626704 \\
\hline $\mathrm{Cl}$ & -1.618790 & 0.704380 & 2.863627 \\
\hline $\mathrm{C}$ & -4.544699 & 3.958220 & -0.051275 \\
\hline $\mathrm{C}$ & -4.604437 & 4.809555 & -1.159000 \\
\hline $\mathrm{C}$ & -5.555871 & 4.078859 & 0.907990 \\
\hline C & -5.616990 & 5.754510 & -1.306306 \\
\hline C & -6.582960 & 5.011316 & 0.777930 \\
\hline C & -6.610362 & 5.852538 & -0.333257 \\
\hline C & 2.410493 & 4.034522 & 0.756227 \\
\hline C & 2.901978 & 4.426311 & -0.490709 \\
\hline C & 2.994985 & 4.612126 & 1.886089 \\
\hline $\mathrm{C}$ & 3.963648 & 5.315620 & -0.621576 \\
\hline C & 4.045701 & 5.522452 & 1.782015 \\
\hline C & 4.533100 & 5.871336 & 0.522494 \\
\hline $\mathrm{C}$ & 2.152743 & -2.791472 & 2.352520 \\
\hline C & 1.803494 & -3.517972 & 3.498145 \\
\hline $\mathrm{C}$ & 3.387865 & -3.090454 & 1.767916 \\
\hline C & 2.634732 & -4.500679 & 4.030852 \\
\hline C & 4.237914 & -4.063358 & 2.284653 \\
\hline C & 3.857616 & -4.774546 & 3.420529 \\
\hline C & -4.192784 & -3.006483 & -0.541920 \\
\hline C & -5.340587 & -3.309572 & 0.197354 \\
\hline C & -3.942122 & -3.776763 & -1.680856 \\
\hline $\mathrm{C}$ & -6.209601 & -4.332015 & -0.178871 \\
\hline C & -4.794039 & -4.805369 & -2.072861 \\
\hline $\mathrm{C}$ & -5.929714 & -5.087409 & -1.31654 \\
\hline
\end{tabular}




\begin{tabular}{|c|c|c|c|}
\hline$F$ & -3.665978 & 4.732025 & -2.112093 \\
\hline $\mathrm{F}$ & -5.647135 & 6.558356 & -2.374266 \\
\hline $\mathrm{F}$ & -7.587764 & 6.751039 & -0.465989 \\
\hline $\mathrm{F}$ & -7.536871 & 5.106428 & 1.708917 \\
\hline F & -5.554914 & 3.280024 & 1.980321 \\
\hline $\mathrm{F}$ & -5.631946 & -2.603139 & 1.295140 \\
\hline F & -7.299751 & -4.598450 & 0.546064 \\
\hline F & -6.748487 & -6.074653 & -1.682832 \\
\hline $\mathrm{F}$ & -4.517468 & -5.536009 & -3.161898 \\
\hline F & -2.849475 & -3.545522 & -2.421151 \\
\hline F & 0.636354 & -3.283027 & 4.107378 \\
\hline $\mathrm{F}$ & 2.270284 & -5.176882 & 5.124172 \\
\hline F & 4.662548 & -5.710921 & 3.925108 \\
\hline F & 5.411367 & -4.316748 & 1.693608 \\
\hline $\mathrm{F}$ & 3.796203 & -2.424443 & 0.678435 \\
\hline F & 2.387856 & 3.885422 & -1.607540 \\
\hline F & 4.461823 & 5.603147 & -1.829466 \\
\hline F & 5.552417 & 6.724598 & 0.414374 \\
\hline F & 4.592874 & 6.056565 & 2.877568 \\
\hline F & 2.564682 & 4.271512 & 3.106096 \\
\hline O & -0.642728 & 0.436302 & -1.119662 \\
\hline $\mathrm{C}$ & 0.807568 & -4.116650 & -2.216423 \\
\hline C & 0.602599 & -2.775781 & -2.019915 \\
\hline $\mathrm{C}$ & 1.718864 & -1.900825 & -1.956581 \\
\hline C & 3.058136 & -2.415294 & -2.124692 \\
\hline C & 3.195518 & -3.818550 & -2.321396 \\
\hline C & 2.105154 & -4.653757 & -2.362715 \\
\hline $\mathrm{H}$ & -0.402064 & -2.384366 & -1.908967 \\
\hline C & 1.592969 & -0.524506 & -1.696596 \\
\hline C & 4.167417 & -1.519991 & -2.027811 \\
\hline $\mathrm{H}$ & 4.183721 & -4.251920 & -2.423705 \\
\hline $\mathrm{H}$ & 2.225081 & -5.722136 & -2.501989 \\
\hline $\mathrm{C}$ & 3.951010 & -0.170919 & -1.759361 \\
\hline C & 2.638021 & 0.347471 & -1.630244 \\
\hline O & -0.306554 & -4.949279 & -2.345967 \\
\hline C & -1.654882 & -6.820958 & -1.854786 \\
\hline $\mathrm{H}$ & -2.098269 & -7.254120 & -0.955463 \\
\hline $\mathrm{H}$ & -2.388879 & -6.245418 & -2.418340 \\
\hline $\mathrm{H}$ & -1.309769 & -7.645487 & -2.490049 \\
\hline C & -0.462634 & -5.981938 & -1.466895 \\
\hline O & 0.270318 & -6.193083 & -0.52892 \\
\hline $\mathrm{C}$ & 5.188943 & 0.668512 & -1.625163 \\
\hline $\mathrm{C}$ & 6.117688 & 0.426424 & -2.843429 \\
\hline $\mathrm{C}$ & 5.126695 & 2.194900 & -1.47317 \\
\hline
\end{tabular}




$\begin{array}{lrrr}\mathrm{H} & 4.805406 & 2.486157 & -0.469625 \\ \mathrm{C} & 6.573713 & 2.637449 & -1.787938 \\ \mathrm{H} & 7.176710 & 2.757745 & -0.879432 \\ \mathrm{C} & 7.197606 & 1.485245 & -2.602880 \\ \mathrm{O} & 8.351019 & 1.427213 & -2.967827 \\ \mathrm{H} & 6.639327 & 3.583698 & -2.335134 \\ \mathrm{H} & 4.420290 & 2.637853 & -2.180084 \\ \mathrm{C} & 6.580610 & -1.042112 & -2.869423 \\ \mathrm{C} & 5.615025 & -1.980923 & -2.079238 \\ \mathrm{H} & 5.962348 & -2.052946 & -1.037629 \\ \mathrm{H} & 5.704780 & -2.989553 & -2.486835 \\ \mathrm{H} & 2.477476 & 1.403865 & -1.446827 \\ \mathrm{H} & 0.411454 & -0.049739 & -1.383122 \\ \mathrm{H} & 6.652149 & -1.384823 & -3.906665 \\ \mathrm{H} & 7.588449 & -1.126837 & -2.448470 \\ \mathrm{C} & 5.430250 & 0.799966 & -4.180490 \\ \mathrm{H} & 6.150916 & 0.698984 & -4.998698 \\ \mathrm{H} & 4.587850 & 0.128223 & -4.371724 \\ \mathrm{H} & 5.050651 & 1.827351 & -4.191335 \\ \mathrm{H} & 5.739842 & 0.294929 & -0.743288\end{array}$

\section{Structure: ${ }^{1} \mathrm{TS} 2 \mathrm{~b}$}

$\begin{array}{lrrr}\mathrm{C} & 1.214970 & -2.614078 & -1.368752 \\ \mathrm{C} & 2.331588 & -3.518908 & -1.250829 \\ \mathrm{C} & 3.415206 & -2.773829 & -0.911651 \\ \mathrm{C} & 2.980063 & -1.399176 & -0.846198 \\ \mathrm{~N} & 1.631364 & -1.327760 & -1.111074 \\ \mathrm{H} & 2.288733 & -4.586538 & -1.408125 \\ \mathrm{H} & 4.417544 & -3.122685 & -0.717269 \\ \mathrm{C} & 2.177523 & 2.834373 & -0.772703 \\ \mathrm{C} & 3.537043 & 3.263205 & -0.541985 \\ \mathrm{C} & 4.293616 & 2.142384 & -0.424457 \\ \mathrm{C} & 3.406161 & 1.015933 & -0.590499 \\ \mathrm{~N} & 2.124127 & 1.461283 & -0.797082 \\ \mathrm{H} & 3.861777 & 4.290867 & -0.471137 \\ \mathrm{H} & 5.358644 & 2.075515 & -0.257989 \\ \mathrm{C} & -2.450376 & -0.357613 & -1.590078 \\ \mathrm{C} & -3.326044 & -1.469977 & -1.881313 \\ \mathrm{C} & -2.544654 & -2.577698 & -1.981956 \\ \mathrm{C} & -1.184567 & -2.153773 & -1.743155 \\ \mathrm{~N} & -1.154161 & -0.802395 & -1.526931 \\ \mathrm{H} & -4.396520 & -1.409943 & -2.005736 \\ \mathrm{H} & -2.857014 & -3.590987 & -2.187958\end{array}$




\begin{tabular}{|c|c|c|c|}
\hline $\mathrm{C}$ & -2.025208 & 2.053524 & -1.225337 \\
\hline C & -2.472833 & 3.430476 & -1.204496 \\
\hline C & -1.361215 & 4.203969 & -1.122810 \\
\hline C & -0.229540 & 3.304217 & -1.058479 \\
\hline $\mathrm{N}$ & -0.662186 & 2.010607 & -1.137453 \\
\hline $\mathrm{H}$ & -3.501127 & 3.753023 & -1.280963 \\
\hline C & -0.088186 & -3.017764 & -1.663993 \\
\hline C & 1.098612 & 3.710325 & -0.907146 \\
\hline C & 3.821908 & -0.317841 & -0.565677 \\
\hline $\mathrm{C}$ & -2.878802 & 0.958596 & -1.384735 \\
\hline Mn & 0.483708 & 0.323411 & -1.069251 \\
\hline $\mathrm{Cl}$ & 0.765417 & 0.579978 & -3.355163 \\
\hline $\mathrm{C}$ & -4.340921 & 1.213072 & -1.250962 \\
\hline $\mathrm{C}$ & -4.860301 & 1.610984 & -0.012482 \\
\hline C & -5.247786 & 1.057919 & -2.303277 \\
\hline C & -6.219857 & 1.833719 & 0.175425 \\
\hline C & -6.617129 & 1.257682 & -2.132612 \\
\hline C & -7.100769 & 1.647000 & -0.885447 \\
\hline $\mathrm{C}$ & -0.340049 & -4.476125 & -1.842244 \\
\hline $\mathrm{C}$ & -0.274288 & -5.357029 & -0.758728 \\
\hline C & -0.658230 & -5.019273 & -3.090431 \\
\hline C & -0.505768 & -6.722222 & -0.899823 \\
\hline $\mathrm{C}$ & -0.901752 & -6.381593 & -3.257141 \\
\hline C & -0.821920 & -7.234350 & -2.157478 \\
\hline C & 5.244179 & -0.604377 & -0.219360 \\
\hline C & 6.126197 & -1.182532 & -1.139319 \\
\hline C & 5.752211 & -0.324182 & 1.054428 \\
\hline C & 7.439869 & -1.500296 & -0.803206 \\
\hline C & 7.060758 & -0.631490 & 1.414252 \\
\hline C & 7.907535 & -1.223934 & 0.480264 \\
\hline C & 1.379495 & 5.173844 & -0.855620 \\
\hline $\mathrm{C}$ & 2.048222 & 5.819962 & -1.900185 \\
\hline $\mathrm{C}$ & 0.987370 & 5.954528 & 0.235785 \\
\hline C & 2.318472 & 7.186473 & -1.865048 \\
\hline C & 1.238809 & 7.323419 & 0.289492 \\
\hline C & 1.909177 & 7.939529 & -0.765880 \\
\hline $\mathrm{F}$ & -4.047139 & 1.774144 & 1.037101 \\
\hline $\mathrm{F}$ & -6.691412 & 2.196108 & 1.376710 \\
\hline $\mathrm{F}$ & -8.414433 & 1.824903 & -0.697261 \\
\hline $\mathrm{F}$ & -7.463689 & 1.084084 & -3.152606 \\
\hline $\mathrm{F}$ & -4.811004 & 0.681872 & -3.511906 \\
\hline $\mathrm{F}$ & 2.453856 & 5.120404 & -2.965537 \\
\hline $\mathrm{F}$ & 2.961078 & 7.778927 & -2.876655 \\
\hline $\mathrm{F}$ & 2.159609 & 9.250107 & -0.723029 \\
\hline
\end{tabular}




$$
\begin{aligned}
& \begin{array}{lll}
0.849129 & 8.046304 & 1.345399
\end{array} \\
& \begin{array}{llll}
5.707535 & -1.463509 & -2.380660
\end{array} \\
& 8.254840-2.060804-1.703438 \\
& \begin{array}{lll}
9.166002 & -1.521365 & 0.811291
\end{array} \\
& \begin{array}{lll}
7.509124 & -0.353517 & 2.642053
\end{array} \\
& \begin{array}{lll}
4.981276 & 0.277637 & 1.971299
\end{array} \\
& \begin{array}{llll}
0.022993 & -4.889813 & 0.467661
\end{array} \\
& \begin{array}{lll}
-0.434185 & -7.539394 & 0.156313
\end{array} \\
& \begin{array}{lll}
-1.050884 & -8.540440 & -2.306985
\end{array} \\
& \begin{array}{lll}
-1.206222 & -6.875771 & -4.460677
\end{array} \\
& -0.745037-4.222419-4.160442 \\
& \begin{array}{lll}
-5.019984 & -0.806120 & 2.464787
\end{array} \\
& \begin{array}{lll}
-4.440185 & -0.482104 & 3.674558
\end{array} \\
& \begin{array}{lll}
-3.052694 & -0.717566 & 3.864033
\end{array} \\
& \begin{array}{lll}
-2.248191 & -1.268193 & 2.816927
\end{array} \\
& \begin{array}{lll}
-2.890342 & -1.566532 & 1.589981
\end{array} \\
& \begin{array}{lll}
-4.240229 & -1.354794 & 1.423913
\end{array} \\
& \begin{array}{lll}
-5.026934 & -0.059404 & 4.476186
\end{array} \\
& \begin{array}{lll}
-2.443481 & -0.408886 & 5.109433
\end{array} \\
& \begin{array}{lll}
-0.840369 & -1.500844 & 3.041995
\end{array} \\
& \begin{array}{lll}
-2.327944 & -1.969454 & 0.758382
\end{array} \\
& \begin{array}{lll}
-4.726441 & -1.598907 & 0.486596
\end{array} \\
& \begin{array}{lll}
-0.292169 & -1.207229 & 4.317090
\end{array} \\
& \begin{array}{lll}
-1.103325 & -0.661742 & 5.327082
\end{array} \\
& \begin{array}{lll}
-6.353011 & -0.648199 & 2.107011
\end{array} \\
& \begin{array}{lll}
-8.655009 & -0.188081 & 2.187862
\end{array} \\
& \begin{array}{lll}
-9.463501 & 0.033297 & 2.885352
\end{array} \\
& \begin{array}{lll}
-8.628062 & 0.577113 & 1.407026
\end{array} \\
& \begin{array}{lll}
-8.824792 & -1.154696 & 1.704094
\end{array} \\
& \begin{array}{lll}
-7.346951 & -0.197394 & 2.938675
\end{array} \\
& \begin{array}{lll}
-7.189354 & 0.133582 & 4.087786
\end{array} \\
& \begin{array}{lll}
1.142909 & -1.586226 & 4.579007
\end{array} \\
& \begin{array}{lll}
2.017590 & -1.387692 & 3.326845
\end{array} \\
& \begin{array}{lll}
1.962743 & -0.956323 & 5.722979
\end{array} \\
& \begin{array}{lll}
1.650471 & -1.307779 & 6.711047
\end{array} \\
& 3.410425-1.390122 \quad 5.373229 \\
& 3.709974-2.294730 \quad 5.915993 \\
& \begin{array}{llll}
3.401396 & -1.729147 & 3.868181
\end{array} \\
& \begin{array}{lll}
4.327638 & -2.194013 & 3.239059
\end{array} \\
& \begin{array}{lll}
2.082387 & 0.098685 & 2.884591
\end{array} \\
& \begin{array}{lll}
2.595080 & 0.175385 & 1.927204
\end{array} \\
& \begin{array}{lll}
1.085204 & 0.527482 & 2.768502
\end{array} \\
& \begin{array}{lll}
2.633692 & 0.713900 & 3.603359
\end{array} \\
& \begin{array}{lll}
4.169842 & -0.630647 & 5.585802
\end{array}
\end{aligned}
$$


$\mathrm{H}$

C

C

$\mathrm{H}$

$\mathrm{H}$

$\mathrm{H}$

$\mathrm{H}$

$\mathrm{H}$

$\mathrm{H}$

$\mathrm{H}$

F

O

$\mathrm{H}$

$\begin{array}{rrr}1.861871 & 0.134070 & 5.716048 \\ 1.475879 & -2.293216 & 2.228855 \\ 0.019253 & -1.945465 & 1.942318 \\ 1.536320 & -3.343950 & 2.551002 \\ 2.072011 & -2.220114 & 1.320801 \\ -0.445225 & -2.631770 & 1.232953 \\ 0.066759 & -0.915583 & 1.204389 \\ -0.664086 & -0.441797 & 6.295532 \\ -3.054428 & 0.022107 & 5.898278 \\ -1.303517 & 5.282382 & -1.110585 \\ 0.343730 & 5.387136 & 1.265277 \\ 0.105025 & 0.242540 & 0.547335 \\ 1.133325 & -2.674312 & 4.774379\end{array}$

Structure: ${ }^{3}$ TS2b

\begin{tabular}{|c|c|c|c|}
\hline C & -1.743084 & -1.649937 & 1.229859 \\
\hline C & -3.025641 & -1.256028 & 1.754405 \\
\hline $\mathrm{C}$ & -2.938332 & 0.061407 & 2.077581 \\
\hline $\mathrm{C}$ & -1.621142 & 0.507390 & 1.703293 \\
\hline $\mathrm{N}$ & -0.906994 & -0.558617 & 1.200086 \\
\hline $\mathrm{H}$ & -3.872211 & -1.913096 & 1.887014 \\
\hline $\mathrm{H}$ & -3.702261 & 0.681756 & 2.520730 \\
\hline $\mathrm{C}$ & 2.081683 & 2.262560 & 0.397495 \\
\hline C & 1.716886 & 3.635840 & 0.640247 \\
\hline C & 0.476498 & 3.632616 & 1.193226 \\
\hline C & 0.062566 & 2.254295 & 1.292801 \\
\hline $\mathrm{N}$ & 1.052117 & 1.443126 & 0.795302 \\
\hline $\mathrm{H}$ & 2.337560 & 4.491611 & 0.420678 \\
\hline $\mathrm{H}$ & -0.123446 & 4.490973 & 1.452156 \\
\hline $\mathrm{C}$ & 2.072112 & -3.412435 & 0.313934 \\
\hline $\mathrm{C}$ & 1.623906 & -4.785997 & 0.300846 \\
\hline C & 0.272031 & -4.765248 & 0.430565 \\
\hline $\mathrm{C}$ & -0.113423 & -3.381660 & 0.576427 \\
\hline $\mathrm{N}$ & 0.994782 & -2.583293 & 0.497211 \\
\hline $\mathrm{H}$ & 2.265223 & -5.648267 & 0.195212 \\
\hline $\mathrm{H}$ & -0.407418 & -5.604688 & 0.465235 \\
\hline $\mathrm{C}$ & 3.775701 & -1.671134 & -0.051144 \\
\hline C & 5.086880 & -1.248873 & -0.483672 \\
\hline C & 5.049024 & 0.103067 & -0.609411 \\
\hline C & 3.725801 & 0.526103 & -0.217286 \\
\hline N & 2.971576 & -0.572398 & 0.102181 \\
\hline $\mathrm{H}$ & 5.914192 & -1.911304 & -0.691986 \\
\hline $\mathrm{H}$ & 5.843833 & 0.760272 & -0.929600 \\
\hline
\end{tabular}




\begin{tabular}{|c|c|c|c|}
\hline $\mathrm{C}$ & -1.408624 & -2.957404 & 0.872595 \\
\hline C & 3.316233 & 1.856791 & -0.112918 \\
\hline $\mathrm{C}$ & -1.183244 & 1.832060 & 1.766645 \\
\hline C & 3.388617 & -3.006060 & 0.090396 \\
\hline Mn & 1.013164 & -0.557420 & 0.613021 \\
\hline $\mathrm{Cl}$ & 1.667188 & -0.750406 & 2.831393 \\
\hline $\mathrm{C}$ & 4.438762 & -4.051004 & -0.071477 \\
\hline C & 4.471719 & -4.896630 & -1.184627 \\
\hline C & 5.447780 & -4.206845 & 0.884938 \\
\hline C & 5.456602 & -5.869061 & -1.340369 \\
\hline C & 6.447570 & -5.167368 & 0.746447 \\
\hline C & 6.448730 & -6.001733 & -0.370190 \\
\hline C & -2.503691 & -3.967028 & 0.808510 \\
\hline C & -3.004380 & -4.371430 & -0.430773 \\
\hline C & -3.096668 & -4.515193 & 1.948678 \\
\hline C & -4.081379 & -5.244168 & -0.545662 \\
\hline C & -4.163765 & -5.408151 & 1.861164 \\
\hline C & -4.658947 & -5.770117 & 0.608417 \\
\hline $\mathrm{C}$ & -2.095074 & 2.869900 & 2.324759 \\
\hline $\mathrm{C}$ & -1.738999 & 3.598451 & 3.466873 \\
\hline C & -3.319629 & 3.189919 & 1.729049 \\
\hline C & -2.552594 & 4.603504 & 3.985014 \\
\hline C & -4.152503 & 4.184921 & 2.231400 \\
\hline C & -3.765064 & 4.897982 & 3.363655 \\
\hline C & 4.278464 & 2.921742 & -0.514925 \\
\hline C & 5.429461 & 3.194710 & 0.231087 \\
\hline C & 4.053737 & 3.700186 & -1.653711 \\
\hline C & 6.325505 & 4.196199 & -0.138309 \\
\hline C & 4.932141 & 4.709038 & -2.038357 \\
\hline $\mathrm{C}$ & 6.070551 & 4.961000 & -1.275561 \\
\hline $\mathrm{F}$ & 3.533747 & -4.786303 & -2.135015 \\
\hline $\mathrm{F}$ & 5.461433 & -6.666701 & -2.413520 \\
\hline $\mathrm{F}$ & 7.399733 & -6.927136 & -0.510777 \\
\hline $\mathrm{F}$ & 7.400174 & -5.296147 & 1.674858 \\
\hline $\mathrm{F}$ & 5.471727 & -3.415703 & 1.962566 \\
\hline $\mathrm{F}$ & 5.697096 & 2.479621 & 1.329147 \\
\hline $\mathrm{F}$ & 7.417829 & 4.434245 & 0.593215 \\
\hline $\mathrm{F}$ & 6.915186 & 5.928952 & -1.634745 \\
\hline $\mathrm{F}$ & 4.678608 & 5.449309 & -3.126563 \\
\hline $\mathrm{F}$ & 2.959420 & 3.497756 & -2.400457 \\
\hline $\mathrm{F}$ & -0.581300 & 3.344153 & 4.086508 \\
\hline $\mathrm{F}$ & -2.181377 & 5.281309 & 5.075079 \\
\hline $\mathrm{F}$ & -4.553423 & 5.855701 & 3.854273 \\
\hline & -5.316534 & 4.457514 & 1.630216 \\
\hline
\end{tabular}




\begin{tabular}{|c|c|c|}
\hline-3.735404 & 2.522103 & 0.643160 \\
\hline-2.482380 & -3.858457 & -1.557591 \\
\hline-4.585619 & -5.543852 & -1.748093 \\
\hline-5.693306 & -6.607008 & 0.516004 \\
\hline-4.71 & -5.913725 & 2.966254 \\
\hline-2.658345 & -4.162700 & 3.162336 \\
\hline 0.65233 & -0.447419 & -1.111511 \\
\hline-0.7134 & 4.091042 & -2.226 \\
\hline-0.538 & 2.747239 & -2.021760 \\
\hline-1.67 & 1.89720 & -1.966 \\
\hline-3.00 & 2.4383 & -2.15 \\
\hline-3.10 & 3.842688 & -2.359292 \\
\hline-1.99 & 4.654083 & -2.391 \\
\hline 0.45 & 2.335824 & -1.89 \\
\hline-1.59 & 0.520080 & -1.70 \\
\hline-4 & 1.56 & -2.0 \\
\hline-4.08 & 4.295728 & $-2.4 \varepsilon$ \\
\hline-2.09 & 5.723582 & -2.5 \\
\hline-3. & 0.21 & -1.7 \\
\hline-2.65 & -0.331437 & $-1 \cdot 6$ \\
\hline 0.41 & 4.897689 & -2.34 \\
\hline 1.80 & 6.74 & -1.8 \\
\hline 2.24 & & -0.9 \\
\hline 2.52 & 6.151289 & -2.413129 \\
\hline 1.48 & 7.580749 & -2.49 \\
\hline 0.58 & 5.942463 & -1.4 \\
\hline-0.16 & 6.186319 & -0.56 \\
\hline-5.20 & -0.591592 & -1.639703 \\
\hline-6.12 & -0.337349 & -2.8 \\
\hline-5.18 & -2.11 & $-1 \cdot 4$ \\
\hline-4.87 & -2.407787 & -0.464593 \\
\hline-6.639844 & -2.527432 & -1.794092 \\
\hline-7.25 & -2.626585 & -0.8886 \\
\hline-7.23082 & -1.367031 & -2.621719 \\
\hline-8.380476 & -1.283510 & -2.993430 \\
\hline-6.724885 & -3.475901 & -2.334696 \\
\hline-4.483812 & -2.584641 & -2.170506 \\
\hline-6.550154 & 1.141894 & -2.906650 \\
\hline-5.568047 & 2.063105 & -2.116152 \\
\hline-5.919591 & 2.149748 & -1.077183 \\
\hline-5.631947 & 3.070997 & -2.530275 \\
\hline-2.514597 & -1.388087 & -1.42611 \\
\hline-0.347 & -0.006582 & -1.38 \\
\hline-6.604 & 1.477819 & -3.9 \\
\hline
\end{tabular}




-7.558928
-5.438273
-6.151530
-4.578690
-5.083942
-5.754125

$-7.558928$

1.255256

$-0.739798$

$-0.628832$

$-0.090486$

$-1.776256$

$-0.196267$
$-2.494774$

$-4.194690$

$-5.018076$

$-4.387425$

$-4.193819$

$-0.764354$

\section{Structure: ${ }^{5} \mathrm{TS} 2 \mathrm{~b}$}

C

C

C

C

$\mathrm{N}$

$\mathrm{H}$

$\mathrm{H}$

C

C

C

C

$\mathrm{N}$

$\mathrm{H}$

$\mathrm{H}$

C

C

C

C

N

$\mathrm{H}$

$\mathrm{H}$

C

C

C

C

$\mathrm{N}$

$\mathrm{H}$

C

C

$\mathrm{C}$

$\mathrm{C}$

$\mathrm{Mn}$

$\mathrm{Cl}$

C

C
$1.234105-2.481943-1.557618$

$2.340314-3.404902-1.466294$

$3.401562-2.708155-0.983507$

$2.966739-1.340920-0.816525$

$1.644988-1.233888-1.161740$

$2.302241-4.452247-1.726721$

$\begin{array}{lll}4.386159 & -3.084562 & -0.754557\end{array}$

$\begin{array}{lll}2.239019 & 2.902645 & -0.657063\end{array}$

$\begin{array}{llll}3.590007 & 3.295669 & -0.325309\end{array}$

$\begin{array}{lll}4.302948 & 2.155318 & -0.145054\end{array}$

$\begin{array}{lll}3.405615 & 1.051028 & -0.393772\end{array}$

$2.152205 \quad 1.537379-0.693728$

$\begin{array}{lll}3.933432 & 4.315011 & -0.226952\end{array}$

$\begin{array}{lll}5.348916 & 2.059272 & 0.105978\end{array}$

$\begin{array}{lll}-2.430355 & -0.207669 & -1.574172\end{array}$

$\begin{array}{lll}-3.311240 & -1.296482 & -1.927790\end{array}$

$\begin{array}{lll}-2.527824 & -2.386091 & -2.148267\end{array}$

$\begin{array}{lll}-1.162517 & -1.977420 & -1.913606\end{array}$

$\begin{array}{lll}-1.135019 & -0.647218 & -1.585838\end{array}$

$\begin{array}{lll}-4.385031 & -1.231859 & -2.015549\end{array}$

$\begin{array}{lll}-2.842179 & -3.381141 & -2.427038\end{array}$

$\begin{array}{lll}-1.974196 & 2.173170 & -1.100611\end{array}$

$\begin{array}{llll}-2.398185 & 3.551976 & -1.042375\end{array}$

$\begin{array}{lll}-1.275043 & 4.314488 & -1.008126\end{array}$

$\begin{array}{lll}-0.149323 & 3.410728 & -0.988805\end{array}$

$\begin{array}{lll}-0.601804 & 2.115970 & -1.064832\end{array}$

$\begin{array}{lll}-3.425047 & 3.886539 & -1.070876\end{array}$

$\begin{array}{lll}-0.066182 & -2.845297 & -1.917407\end{array}$

$\begin{array}{lll}1.181814 & 3.800118 & -0.838420\end{array}$

$3.796443-0.287271-0.400940$

$\begin{array}{lll}-2.843820 & 1.097300 & -1.276749\end{array}$

$\begin{array}{lll}0.502613 & 0.453563 & -1.093867\end{array}$

$\begin{array}{lll}0.904763 & 0.702980 & -3.390164\end{array}$

$\begin{array}{lll}-4.299581 & 1.358498 & -1.101454\end{array}$

$\begin{array}{lll}-4.796701 & 1.692846 & 0.164779\end{array}$ 


\begin{tabular}{|c|c|c|}
\hline-5.221450 & 1.270420 & -2.148742 \\
\hline-6.151831 & 1.919065 & 0.382497 \\
\hline-6.585414 & 1.477855 & -1.949654 \\
\hline 7. & & -0.6765 \\
\hline 0.322 & -4.275921 & -2.248523 \\
\hline 0.276 & -5.266102 & $-1.262^{\prime}$ \\
\hline 0 . & -4.67 & -3.5 \\
\hline 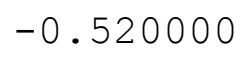 & -6.6060 & -1.5 \\
\hline 0.88 & -6.01 & -3.8 \\
\hline 0 . & -6.9 & $-2 . \varepsilon$ \\
\hline 5 . & -0.6 & \\
\hline 6.12 & $-1 \cdot 1$ & -0 . \\
\hline & -0.4 & \\
\hline 7. & -1.5 & -0 . \\
\hline 6. & -0.7 & \\
\hline & $-1 \cdot 3$ & \\
\hline 1 & 5.2 & -0 \\
\hline & 5. & -1 \\
\hline & & \\
\hline 2 . & 7.2 & -1 \\
\hline & 7.4 & \\
\hline & & -0 \\
\hline-3.96 & 1.7 & 1.2 \\
\hline-6 . & 2.2 & 1 . \\
\hline-8 & 1.5 & -0 . \\
\hline-7. & & \\
\hline-4 . & 0.9 & -3 \\
\hline 2 . & 5. & -2 \\
\hline & & \\
\hline 2 . & 9.3 & -0 \\
\hline 0. & 8.2 & \\
\hline 5.8 & $-1 \cdot 3$ & -2 \\
\hline 8.2 & $-2 \cdot 0$ & -1 \\
\hline 9. & -1.6 & \\
\hline 7.23 & -0.5 & \\
\hline $4.7^{7}$ & & \\
\hline 0.00 & -4.93365 & \\
\hline-0.468404 & -7.530712 & -0.586 \\
\hline-1.06611 & -8.259964 & -3.146 \\
\hline-1.184692 & -6.374749 & -5.1142 \\
\hline-0.705147 & -3.773406 & -4.5296 \\
\hline-5.113127 & -0.925557 & \\
\hline-4.5415 & -0.8 & \\
\hline-3.16 & $-1 \cdot 1$ & 3.9 \\
\hline
\end{tabular}




\begin{tabular}{|c|c|c|c|}
\hline C & -2.365827 & -1.539808 & 2.809254 \\
\hline C & -3.000016 & -1.614905 & 1.543506 \\
\hline $\mathrm{C}$ & -4.338698 & -1.329624 & 1.399677 \\
\hline $\mathrm{H}$ & -5.126148 & -0.514941 & 4.614697 \\
\hline $\mathrm{C}$ & -2.563236 & -1.057817 & 5.208646 \\
\hline $\mathrm{C}$ & -0.971762 & -1.845721 & 2.996403 \\
\hline $\mathrm{H}$ & -2.435868 & -1.900455 & 0.665949 \\
\hline $\mathrm{H}$ & -4.820230 & -1.401781 & 0.431217 \\
\hline $\mathrm{C}$ & -0.423855 & -1.764224 & 4.298782 \\
\hline $\mathrm{C}$ & -1.230672 & -1.372994 & 5.383691 \\
\hline O & -6.438305 & -0.677729 & 2.172798 \\
\hline C & -8.712981 & -0.107723 & 2.301135 \\
\hline $\mathrm{H}$ & -9.500726 & 0.138813 & 3.013706 \\
\hline $\mathrm{H}$ & -8.644416 & 0.678776 & 1.544795 \\
\hline $\mathrm{H}$ & -8.946580 & -1.045609 & 1.787844 \\
\hline $\mathrm{C}$ & -7.402702 & -0.226367 & 3.038292 \\
\hline O & -7.222102 & 0.028259 & 4.203348 \\
\hline $\mathrm{C}$ & 1.013092 & -2.174817 & 4.498474 \\
\hline $\mathrm{C}$ & 1.875919 & -1.802935 & 3.277707 \\
\hline $\mathrm{C}$ & 1.838226 & -1.693717 & 5.709667 \\
\hline $\mathrm{H}$ & 1.538391 & -2.173490 & 6.646389 \\
\hline $\mathrm{C}$ & 3.285843 & -2.064409 & 5.294964 \\
\hline $\mathrm{H}$ & 3.592239 & -3.035331 & 5.703005 \\
\hline $\mathrm{C}$ & 3.268677 & -2.189482 & 3.757328 \\
\hline O & 4.201852 & -2.538932 & 3.064705 \\
\hline $\mathrm{C}$ & 1.917317 & -0.268637 & 3.037603 \\
\hline $\mathrm{H}$ & 2.386330 & -0.057141 & 2.078447 \\
\hline $\mathrm{H}$ & 0.914025 & 0.159657 & 3.010897 \\
\hline $\mathrm{H}$ & 2.491529 & 0.248855 & 3.813335 \\
\hline $\mathrm{H}$ & 4.043691 & -1.338856 & 5.607967 \\
\hline $\mathrm{H}$ & 1.728674 & -0.612421 & 5.844832 \\
\hline $\mathrm{C}$ & 1.332215 & -2.558525 & 2.073218 \\
\hline $\mathrm{C}$ & -0.119233 & -2.142666 & 1.817064 \\
\hline $\mathrm{H}$ & 1.384877 & -3.640547 & 2.261020 \\
\hline $\mathrm{H}$ & 1.935912 & -2.369371 & 1.187983 \\
\hline $\mathrm{H}$ & -0.598837 & -2.809771 & 1.095395 \\
\hline $\mathrm{H}$ & -0.065457 & -1.130259 & 1.224730 \\
\hline $\mathrm{H}$ & -0.790530 & -1.318476 & 6.375025 \\
\hline $\mathrm{H}$ & -3.169635 & -0.746009 & 6.055055 \\
\hline $\mathrm{H}$ & -1.208666 & 5.392002 & -0.989411 \\
\hline $\mathrm{F}$ & 0.389182 & 5.562399 & 1.265340 \\
\hline O & 0.153695 & 0.245979 & 0.593957 \\
\hline $\mathrm{H}$ & 1.011044 & -3.278971 & 4.548403 \\
\hline
\end{tabular}


Structure: ${ }^{3}$ INT3a

\begin{tabular}{|c|c|c|c|}
\hline $\mathrm{C}$ & 1.267358 & 2.162285 & 0.937548 \\
\hline $\mathrm{C}$ & 2.582371 & 2.117976 & 1.526383 \\
\hline $\mathrm{C}$ & 2.756581 & 0.861942 & 2.021075 \\
\hline $\mathrm{C}$ & 1.583114 & 0.099946 & 1.676528 \\
\hline $\mathrm{N}$ & 0.685159 & 0.924427 & 1.036621 \\
\hline $\mathrm{H}$ & 3.257327 & 2.958106 & 1.601605 \\
\hline $\mathrm{H}$ & 3.612244 & 0.480725 & 2.556766 \\
\hline C & -1.553784 & -2.497342 & 0.321366 \\
\hline $\mathrm{C}$ & -0.900331 & -3.741399 & 0.638840 \\
\hline $\mathrm{C}$ & 0.260024 & -3.437358 & 1.278697 \\
\hline $\mathrm{C}$ & 0.342207 & -1.998669 & 1.344590 \\
\hline $\mathrm{N}$ & -0.769330 & -1.454650 & 0.749288 \\
\hline $\mathrm{H}$ & -1.288455 & -4.723563 & 0.417019 \\
\hline $\mathrm{H}$ & 1.015478 & -4.130824 & 1.614229 \\
\hline $\mathrm{C}$ & -2.873495 & 3.004274 & 0.196538 \\
\hline $\mathrm{C}$ & -2.736107 & 4.439882 & 0.092410 \\
\hline $\mathrm{C}$ & -1.404704 & 4.713901 & 0.079691 \\
\hline $\mathrm{C}$ & -0.716152 & 3.456208 & 0.249189 \\
\hline $\mathrm{N}$ & -1.630496 & 2.438911 & 0.309094 \\
\hline $\mathrm{H}$ & -3.554932 & 5.139480 & 0.014834 \\
\hline $\mathrm{H}$ & -0.923206 & 5.678586 & 0.005408 \\
\hline $\mathrm{C}$ & -4.141345 & 0.910450 & -0.067374 \\
\hline $\mathrm{C}$ & -5.319530 & 0.190119 & -0.489558 \\
\hline $\mathrm{C}$ & -4.949512 & -1.103962 & -0.682452 \\
\hline C & -3.554991 & -1.202972 & -0.322868 \\
\hline $\mathrm{N}$ & -3.091097 & 0.037876 & 0.031945 \\
\hline $\mathrm{H}$ & -6.290820 & 0.633517 & -0.654335 \\
\hline $\mathrm{H}$ & -5.566969 & -1.923986 & -1.018143 \\
\hline $\mathrm{C}$ & 0.653795 & 3.330709 & 0.474555 \\
\hline $\mathrm{C}$ & -2.829030 & -2.394002 & -0.242395 \\
\hline $\mathrm{C}$ & 1.430065 & -1.279144 & 1.854271 \\
\hline $\mathrm{C}$ & -4.074639 & 2.300791 & 0.073996 \\
\hline $\mathrm{Mn}$ & -1.195826 & 0.481828 & 0.503891 \\
\hline $\mathrm{Cl}$ & -1.819042 & 0.611450 & 2.734829 \\
\hline $\mathrm{C}$ & -5.352167 & 3.062968 & -0.001014 \\
\hline $\mathrm{C}$ & -5.670132 & 3.877717 & -1.092088 \\
\hline $\mathrm{C}$ & -6.299874 & 2.962623 & 1.023819 \\
\hline C & -6.870384 & 4.581011 & -1.16002 \\
\hline $\mathrm{C}$ & -7.510184 & 3.651116 & 0.973957 \\
\hline C & -7.793521 & 4.464221 & -0.122096 \\
\hline $\mathrm{C}$ & 1.529056 & 4.524923 & 0.292120 \\
\hline C & 2.236050 & 4.697256 & -0.89910 \\
\hline
\end{tabular}




\begin{tabular}{|c|c|c|c|}
\hline C & 1.741195 & 5.461536 & 1.306287 \\
\hline C & 3.163186 & 5.720836 & -1.068070 \\
\hline $\mathrm{C}$ & 2.643132 & 6.513749 & 1.152541 \\
\hline C & 3.362964 & 6.636229 & -0.036159 \\
\hline C & 2.490336 & -2.050033 & 2.560701 \\
\hline $\mathrm{C}$ & 2.193288 & -2.777027 & 3.721722 \\
\hline $\mathrm{C}$ & 3.806465 & -2.124843 & 2.094414 \\
\hline $\mathrm{C}$ & 3.153138 & -3.545046 & 4.377573 \\
\hline $\mathrm{C}$ & 4.783955 & -2.880573 & 2.733126 \\
\hline $\mathrm{C}$ & 4.454814 & -3.597755 & 3.880847 \\
\hline C & -3.472343 & -3.654774 & -0.707915 \\
\hline C & -4.539145 & -4.241419 & -0.020004 \\
\hline $\mathrm{C}$ & -3.005452 & -4.314439 & -1.849069 \\
\hline $\mathrm{C}$ & -5.130638 & -5.427380 & -0.451178 \\
\hline $\mathrm{C}$ & -3.582280 & -5.498790 & -2.298169 \\
\hline C & -4.643292 & -6.062046 & -1.592503 \\
\hline F & -4.805658 & 4.000914 & -2.108953 \\
\hline $\mathrm{F}$ & -7.145603 & 5.356336 & -2.214212 \\
\hline F & -8.948351 & 5.130320 & -0.178284 \\
\hline $\mathrm{F}$ & -8.396898 & 3.540247 & 1.967592 \\
\hline $\mathrm{F}$ & -6.055222 & 2.186153 & 2.083989 \\
\hline $\mathrm{F}$ & -5.022437 & -3.657944 & 1.082574 \\
\hline $\mathrm{F}$ & -6.149011 & -5.964622 & 0.226568 \\
\hline $\mathrm{F}$ & -5.193663 & -7.202758 & -2.011277 \\
\hline $\mathrm{F}$ & -3.106107 & -6.113306 & -3.390221 \\
\hline $\mathrm{F}$ & -1.974839 & -3.813155 & -2.543301 \\
\hline F & 0.955389 & -2.754455 & 4.225601 \\
\hline F & 2.835684 & -4.227387 & 5.481441 \\
\hline F & 5.380394 & -4.328138 & 4.504406 \\
\hline F & 6.029395 & -2.920911 & 2.242929 \\
\hline F & 4.170509 & -1.447316 & 0.996050 \\
\hline F & 2.056927 & 3.833113 & -1.912259 \\
\hline F & 3.873339 & 5.812900 & -2.198771 \\
\hline F & 4.244971 & 7.625887 & -0.182637 \\
\hline F & 2.834364 & 7.394713 & 2.138504 \\
\hline F & 1.085320 & 5.346053 & 2.465904 \\
\hline O & -0.771208 & 0.393096 & -1.225887 \\
\hline C & 1.649626 & -4.193582 & -2.007654 \\
\hline $\mathrm{C}$ & 1.245833 & -2.883925 & -1.991400 \\
\hline $\mathrm{C}$ & 2.222819 & -1.858038 & -1.903140 \\
\hline $\mathrm{C}$ & 3.632183 & -2.182961 & -1.851406 \\
\hline $\mathrm{C}$ & 3.974148 & -3.564686 & -1.872513 \\
\hline $\mathrm{C}$ & 3.015259 & -4.547389 & -1.944519 \\
\hline $\mathrm{H}$ & 0.192301 & -2.633096 & -2.031447 \\
\hline
\end{tabular}




\begin{tabular}{|c|c|c|c|}
\hline C & 1.912714 & -0.497725 & -1.823190 \\
\hline $\mathrm{C}$ & 4.590669 & -1.127116 & -1.733427 \\
\hline $\mathrm{H}$ & 5.015193 & -3.861381 & -1.815771 \\
\hline $\mathrm{H}$ & 3.292216 & -5.595569 & -1.949307 \\
\hline $\mathrm{C}$ & 4.165770 & 0.195334 & -1.628834 \\
\hline C & 2.785547 & 0.531981 & -1.701845 \\
\hline O & 0.680598 & -5.189001 & -2.160027 \\
\hline $\mathrm{C}$ & -0.434906 & -7.195568 & -1.612843 \\
\hline $\mathrm{H}$ & -0.952628 & -7.577461 & -0.730039 \\
\hline $\mathrm{H}$ & -1.141916 & -6.813030 & -2.348709 \\
\hline $\mathrm{H}$ & 0.113042 & -8.030148 & -2.066425 \\
\hline $\mathrm{C}$ & 0.560341 & -6.143519 & -1.189830 \\
\hline O & 1.182388 & -6.140798 & -0.153285 \\
\hline $\mathrm{C}$ & 5.249453 & 1.216072 & -1.427406 \\
\hline C & 6.373152 & 1.001148 & -2.474669 \\
\hline $\mathrm{C}$ & 4.963832 & 2.725903 & -1.462237 \\
\hline $\mathrm{H}$ & 4.459993 & 3.064640 & -0.553162 \\
\hline $\mathrm{C}$ & 6.370399 & 3.343737 & -1.627511 \\
\hline $\mathrm{H}$ & 6.809904 & 3.635485 & -0.665515 \\
\hline $\mathrm{C}$ & 7.254598 & 2.225554 & -2.214790 \\
\hline O & 8.446898 & 2.303353 & -2.414082 \\
\hline $\mathrm{H}$ & 6.391414 & 4.234550 & -2.263813 \\
\hline $\mathrm{H}$ & 4.319703 & 2.990512 & -2.304116 \\
\hline $\mathrm{C}$ & 7.029284 & -0.375359 & -2.270987 \\
\hline $\mathrm{C}$ & 6.078561 & -1.388505 & -1.557459 \\
\hline $\mathrm{H}$ & 6.276703 & -1.364949 & -0.475915 \\
\hline $\mathrm{H}$ & 6.347906 & -2.396034 & -1.880835 \\
\hline $\mathrm{H}$ & 2.460824 & 1.563725 & -1.636644 \\
\hline $\mathrm{H}$ & 0.143142 & 0.031415 & -1.360914 \\
\hline $\mathrm{H}$ & 7.334448 & -0.781462 & -3.240693 \\
\hline $\mathrm{H}$ & 7.946660 & -0.269609 & -1.681852 \\
\hline $\mathrm{C}$ & 5.848528 & 1.140208 & -3.925832 \\
\hline H & 6.691576 & 1.083692 & -4.622340 \\
\hline $\mathrm{H}$ & 5.151235 & 0.328627 & -4.155321 \\
\hline $\mathrm{H}$ & 5.329528 & 2.088671 & -4.100604 \\
\hline & 5.704624 & 1.008532 & -0.44199 \\
\hline
\end{tabular}

\section{Structure: ${ }^{5}$ INT3a}

C
C
C
$\mathrm{N}$

$\begin{array}{lll}1.206059 & 2.214658 & 0.908712 \\ 2.520056 & 2.209418 & 1.502809 \\ 2.722300 & 0.963763 & 2.013096 \\ 1.568755 & 0.169563 & 1.672120 \\ 0.653795 & 0.965606 & 1.021606\end{array}$




\begin{tabular}{|c|c|c|c|}
\hline $\mathrm{H}$ & 3.173952 & 3.066647 & 1.571168 \\
\hline $\mathrm{H}$ & 3.584887 & 0.609464 & 2.556116 \\
\hline $\mathrm{C}$ & -1.493547 & -2.513112 & 0.312212 \\
\hline $\mathrm{C}$ & -0.806946 & -3.738677 & 0.631355 \\
\hline C & 0.341779 & -3.402613 & 1.276356 \\
\hline C & 0.383441 & -1.962095 & 1.343220 \\
\hline $\mathrm{N}$ & -0.740471 & -1.449552 & 0.743000 \\
\hline $\mathrm{H}$ & -1.165657 & -4.731437 & 0.407786 \\
\hline $\mathrm{H}$ & 1.113896 & -4.076072 & 1.614774 \\
\hline $\mathrm{C}$ & -2.958344 & 2.950765 & 0.184091 \\
\hline $\mathrm{C}$ & -2.855448 & 4.388227 & 0.067873 \\
\hline C & -1.530913 & 4.693325 & 0.041154 \\
\hline C & -0.810879 & 3.454293 & 0.216539 \\
\hline $\mathrm{N}$ & -1.701505 & 2.416060 & 0.292366 \\
\hline $\mathrm{H}$ & -3.690967 & 5.067817 & -0.009590 \\
\hline $\mathrm{H}$ & -1.073501 & 5.668551 & -0.046347 \\
\hline C & -4.172025 & 0.824723 & -0.070841 \\
\hline C & -5.330389 & 0.073341 & -0.492698 \\
\hline C & -4.925259 & -1.209680 & -0.690135 \\
\hline $\mathrm{C}$ & -3.528541 & -1.272645 & -0.332094 \\
\hline $\mathrm{N}$ & -3.098155 & -0.019370 & 0.024966 \\
\hline $\mathrm{H}$ & -6.313594 & 0.490558 & -0.655216 \\
\hline $\mathrm{H}$ & -5.520839 & -2.044764 & -1.028097 \\
\hline $\mathrm{C}$ & 0.562837 & 3.363270 & 0.434154 \\
\hline C & -2.771120 & -2.443933 & -0.252695 \\
\hline $\mathrm{C}$ & 1.448889 & -1.212134 & 1.857295 \\
\hline $\mathrm{C}$ & -4.141904 & 2.216466 & 0.070679 \\
\hline $\mathrm{Mn}$ & -1.220870 & 0.475577 & 0.495984 \\
\hline $\mathrm{Cl}$ & -1.827620 & 0.589379 & 2.730201 \\
\hline $\mathrm{C}$ & -5.439723 & 2.944087 & 0.002846 \\
\hline C & -5.786678 & 3.749626 & -1.086249 \\
\hline C & -6.378099 & 2.817882 & 1.033437 \\
\hline $\mathrm{C}$ & -7.006144 & 4.419797 & -1.146634 \\
\hline C & -7.607109 & 3.472935 & 0.991164 \\
\hline $\mathrm{C}$ & -7.919286 & 4.277969 & -0.103007 \\
\hline C & 1.410990 & 4.574062 & 0.235903 \\
\hline C & 2.145895 & 4.726812 & -0.941236 \\
\hline C & 1.573497 & 5.546687 & 1.225058 \\
\hline $\mathrm{C}$ & 3.053284 & 5.766713 & -1.117508 \\
\hline $\mathrm{C}$ & 2.454110 & 6.615417 & 1.062086 \\
\hline C & 3.203675 & 6.718070 & -0.110015 \\
\hline C & 2.522863 & -1.953146 & 2.574685 \\
\hline C & 2.235639 & -2.685955 & 3.734589 \\
\hline C & 3.843942 & -1.995014 & 2.118967 \\
\hline
\end{tabular}




\begin{tabular}{|c|c|c|c|}
\hline C & 3.209973 & -3.428462 & 4.398546 \\
\hline C & 4.835511 & -2.725455 & 2.765068 \\
\hline $\mathrm{C}$ & 4.516213 & -3.449372 & 3.911336 \\
\hline $\mathrm{C}$ & -3.380912 & -3.722221 & -0.715562 \\
\hline C & -4.437487 & -4.330717 & -0.030593 \\
\hline $\mathrm{C}$ & -2.890781 & -4.378241 & -1.849208 \\
\hline $\mathrm{C}$ & -4.998106 & -5.532769 & -0.458325 \\
\hline $\mathrm{C}$ & -3.436922 & -5.578260 & -2.295002 \\
\hline $\mathrm{C}$ & -4.488884 & -6.162362 & -1.592807 \\
\hline $\mathrm{F}$ & -4.932400 & 3.896169 & -2.108690 \\
\hline $\mathrm{F}$ & -7.309195 & 5.187083 & -2.199068 \\
\hline F & -9.092130 & 4.912383 & -0.151946 \\
\hline $\mathrm{F}$ & -8.484283 & 3.337790 & 1.990239 \\
\hline $\mathrm{F}$ & -6.105756 & 2.048397 & 2.091930 \\
\hline $\mathrm{F}$ & -4.941210 & -3.753424 & 1.066130 \\
\hline F & -6.007387 & -6.090098 & 0.216727 \\
\hline $\mathrm{F}$ & -5.009748 & -7.317944 & -2.008439 \\
\hline $\mathrm{F}$ & -2.940263 & -6.187249 & -3.381088 \\
\hline F & -1.867362 & -3.857739 & -2.540293 \\
\hline $\mathrm{F}$ & 0.994008 & -2.694397 & 4.229279 \\
\hline $\mathrm{F}$ & 2.901825 & -4.117092 & 5.501105 \\
\hline $\mathrm{F}$ & 5.455038 & -4.155980 & 4.542387 \\
\hline F & 6.084880 & -2.735612 & 2.282766 \\
\hline $\mathrm{F}$ & 4.198218 & -1.309907 & 1.022219 \\
\hline $\mathrm{F}$ & 2.011549 & 3.831066 & -1.933421 \\
\hline $\mathrm{F}$ & 3.790079 & 5.842126 & -2.232311 \\
\hline F & 4.065605 & 7.724051 & -0.264432 \\
\hline $\mathrm{F}$ & 2.597258 & 7.531044 & 2.024357 \\
\hline F & 0.887921 & 5.451870 & 2.369400 \\
\hline O & -0.781229 & 0.392422 & -1.236548 \\
\hline $\mathrm{C}$ & 1.773855 & -4.208761 & -2.004156 \\
\hline C & 1.342987 & -2.907827 & -2.016655 \\
\hline $\mathrm{C}$ & 2.296203 & -1.860626 & -1.920105 \\
\hline C & 3.710199 & -2.155091 & -1.828212 \\
\hline C & 4.081054 & -3.529626 & -1.820375 \\
\hline $\mathrm{C}$ & 3.144383 & -4.533064 & -1.902231 \\
\hline $\mathrm{H}$ & 0.285574 & -2.680280 & -2.083296 \\
\hline C & 1.955372 & -0.506677 & -1.866346 \\
\hline $\mathrm{C}$ & 4.643374 & -1.077256 & -1.701356 \\
\hline $\mathrm{H}$ & 5.126023 & -3.803471 & -1.731228 \\
\hline $\mathrm{H}$ & 3.442579 & -5.575358 & -1.884726 \\
\hline $\mathrm{C}$ & 4.187291 & 0.236307 & -1.618872 \\
\hline $\mathrm{C}$ & 2.802161 & 0.542820 & -1.731735 \\
\hline 0 & 0.829635 & -5.226799 & -2.164475 \\
\hline
\end{tabular}




\begin{tabular}{|c|c|c|c|}
\hline C & -0.258758 & -7.244909 & -1.609674 \\
\hline $\mathrm{H}$ & -0.758471 & -7.658282 & -0.731065 \\
\hline $\mathrm{H}$ & -0.981752 & -6.878301 & -2.338418 \\
\hline $\mathrm{H}$ & 0.318169 & -8.050050 & -2.080407 \\
\hline C & 0.696942 & -6.161164 & -1.176353 \\
\hline 0 & 1.282806 & -6.120033 & -0.119668 \\
\hline C & 5.241232 & 1.283136 & -1.394965 \\
\hline C & 6.402705 & 1.083213 & -2.402769 \\
\hline C & 4.921539 & 2.785187 & -1.457491 \\
\hline $\mathrm{H}$ & 4.379493 & 3.121377 & -0.569939 \\
\hline C & 6.317690 & 3.434554 & -1.583881 \\
\hline $\mathrm{H}$ & 6.719452 & 3.743466 & -0.610717 \\
\hline $\mathrm{C}$ & 7.245386 & 2.332821 & -2.133274 \\
\hline 0 & 8.440394 & 2.438600 & -2.301190 \\
\hline $\mathrm{H}$ & 6.338722 & 4.320851 & -2.226415 \\
\hline $\mathrm{H}$ & 4.299634 & 3.024661 & -2.323359 \\
\hline $\mathrm{C}$ & 7.086779 & -0.271869 & -2.156763 \\
\hline $\mathrm{C}$ & 6.131275 & -1.310408 & -1.485961 \\
\hline $\mathrm{H}$ & 6.296829 & -1.298297 & -0.398990 \\
\hline $\mathrm{H}$ & 6.427569 & -2.308750 & -1.814585 \\
\hline $\mathrm{H}$ & 2.454543 & 1.568354 & -1.686085 \\
\hline $\mathrm{H}$ & 0.126796 & 0.026813 & -1.350316 \\
\hline $\mathrm{H}$ & 7.455463 & -0.672576 & -3.106391 \\
\hline $\mathrm{H}$ & 7.968182 & -0.135218 & -1.520962 \\
\hline 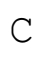 & 5.922370 & 1.187126 & -3.871997 \\
\hline-1 & 6.789180 & 1.144888 & -4.539765 \\
\hline H & 5.255860 & 0.353114 & -4.112052 \\
\hline $\mathrm{H}$ & 5.383153 & 2.117950 & -4.077755 \\
\hline & 5.667993 & 1.096595 & -0.39274 \\
\hline
\end{tabular}

\section{Structure: ${ }^{3}$ INT3b}

C
C
C
C
N
H
H
C
C
C
C
N

$\begin{array}{rrr}-3.146542 & 0.333731 & -1.565269 \\ -4.246822 & -0.582080 & -1.751833 \\ -3.740442 & -1.839154 & -1.642178 \\ -2.317086 & -1.705322 & -1.443574 \\ -1.982179 & -0.376761 & -1.430750 \\ -5.275678 & -0.291647 & -1.906847 \\ -4.276228 & -2.774878 & -1.702203 \\ 1.981864 & -1.773971 & -1.580802 \\ 2.117575 & -3.207337 & -1.663174 \\ 0.880994 & -3.740272 & -1.463637 \\ -0.036856 & -2.637721 & -1.325943 \\ 0.659522 & -1.456097 & -1.387231\end{array}$




\begin{tabular}{|c|c|c|c|}
\hline $\mathrm{H}$ & 3.043982 & -3.734675 & -1.835636 \\
\hline $\mathrm{H}$ & 0.598170 & -4.783288 & -1.459076 \\
\hline C & -0.263474 & 3.272400 & -0.315353 \\
\hline C & -1.210468 & 4.329260 & -0.051447 \\
\hline C & -2.425047 & 3.892976 & -0.478765 \\
\hline C & -2.244478 & 2.545963 & -0.964601 \\
\hline $\mathrm{N}$ & -0.922101 & 2.199376 & -0.863458 \\
\hline $\mathrm{H}$ & -0.970474 & 5.286030 & 0.387908 \\
\hline $\mathrm{H}$ & -3.369263 & 4.417002 & -0.445664 \\
\hline $\mathrm{C}$ & 2.026517 & 2.376740 & -0.511514 \\
\hline C & 3.456377 & 2.557303 & -0.570545 \\
\hline C & 3.987739 & 1.405208 & -1.056453 \\
\hline C & 2.893116 & 0.489235 & -1.263014 \\
\hline $\mathrm{N}$ & 1.711216 & 1.113534 & -0.942881 \\
\hline $\mathrm{H}$ & 3.980525 & 3.459211 & -0.290564 \\
\hline C & -3.289261 & 1.706226 & -1.353834 \\
\hline C & 3.040094 & -0.860580 & -1.590317 \\
\hline C & -1.426114 & -2.770684 & -1.294104 \\
\hline C & 1.118491 & 3.368384 & -0.128253 \\
\hline $\mathrm{Mn}$ & -0.136404 & 0.374806 & -1.158611 \\
\hline $\mathrm{Cl}$ & 0.016977 & 0.746292 & -3.431318 \\
\hline $\mathrm{C}$ & 1.680543 & 4.613638 & 0.463464 \\
\hline $\mathrm{C}$ & 2.342568 & 4.584336 & 1.695778 \\
\hline C & 1.603833 & 5.849376 & -0.188331 \\
\hline C & 2.897774 & 5.726370 & 2.265852 \\
\hline C & 2.144386 & 7.008257 & 0.364921 \\
\hline C & 2.795120 & 6.944611 & 1.595856 \\
\hline C & -4.665778 & 2.278067 & -1.374290 \\
\hline C & -5.115121 & 3.062194 & -2.439002 \\
\hline $\mathrm{C}$ & -5.543535 & 2.064067 & -0.306290 \\
\hline C & -6.397419 & 3.608357 & -2.451951 \\
\hline C & -6.830539 & 2.596555 & -0.299134 \\
\hline $\mathrm{C}$ & -7.254546 & 3.371907 & -1.378483 \\
\hline C & -1.992550 & -4.125720 & -1.042551 \\
\hline $\mathrm{C}$ & -2.602829 & -4.899427 & -2.031709 \\
\hline C & -1.959702 & -4.649333 & 0.253495 \\
\hline C & -3.173152 & -6.138845 & -1.743620 \\
\hline C & -2.533604 & -5.876367 & 0.569128 \\
\hline C & -3.139011 & -6.626946 & -0.437489 \\
\hline C & 4.415943 & -1.359094 & -1.865910 \\
\hline C & 5.101127 & -0.968141 & -3.021020 \\
\hline $\mathrm{C}$ & 5.095666 & -2.187738 & -0.970626 \\
\hline C & 6.406912 & -1.381437 & -3.278553 \\
\hline & 6.395125 & -2.619811 & -1.21161 \\
\hline
\end{tabular}




\begin{tabular}{|c|c|c|c|}
\hline C & 7.056783 & -2.211108 & -2.366643 \\
\hline $\mathrm{F}$ & 2.463250 & 3.426003 & 2.367155 \\
\hline $\mathrm{F}$ & 3.521179 & 5.663515 & 3.446402 \\
\hline $\mathrm{F}$ & 3.319526 & 8.047599 & 2.132268 \\
\hline F & 2.052805 & 8.174773 & -0.280159 \\
\hline $\mathrm{F}$ & 0.996592 & 5.941212 & -1.377357 \\
\hline F & 4.506116 & -0.162641 & -3.906502 \\
\hline F & 7.037048 & -0.990611 & -4.390388 \\
\hline $\mathrm{F}$ & 8.307949 & -2.613830 & -2.598789 \\
\hline F & 7.025029 & -3.403948 & -0.321155 \\
\hline $\mathrm{F}$ & -2.670086 & -4.442589 & -3.288210 \\
\hline$F$ & -3.753975 & -6.860115 & -2.707316 \\
\hline $\mathrm{F}$ & -3.690635 & -7.807752 & -0.151872 \\
\hline F & -2.516728 & -6.324066 & 1.830619 \\
\hline F & -1.385713 & -3.949978 & 1.242371 \\
\hline F & -4.306654 & 3.303930 & -3.476628 \\
\hline $\mathrm{F}$ & -6.810303 & 4.352871 & -3.482453 \\
\hline F & -8.484323 & 3.890254 & -1.382489 \\
\hline F & -7.654575 & 2.379640 & 0.728835 \\
\hline F & -5.150065 & 1.333574 & 0.746519 \\
\hline C & 3.975528 & -1.023647 & 2.811444 \\
\hline C & 3.262361 & -2.156263 & 3.130529 \\
\hline C & 1.862873 & -2.096617 & 3.333541 \\
\hline $\mathrm{C}$ & 1.187853 & -0.837555 & 3.219152 \\
\hline C & 1.961144 & 0.288420 & 2.842417 \\
\hline C & 3.330031 & 0.215920 & 2.641737 \\
\hline $\mathrm{H}$ & 3.788549 & -3.099608 & 3.239065 \\
\hline $\mathrm{C}$ & 1.126106 & -3.271154 & 3.654203 \\
\hline C & -0.255742 & -0.775448 & 3.468484 \\
\hline $\mathrm{H}$ & 1.484770 & 1.249926 & 2.698974 \\
\hline $\mathrm{H}$ & 3.888582 & 1.097584 & 2.357462 \\
\hline C & -0.931073 & -1.993712 & 3.807017 \\
\hline $\mathrm{C}$ & -0.237981 & -3.205011 & 3.880814 \\
\hline O & 5.365011 & -1.180157 & 2.778416 \\
\hline $\mathrm{C}$ & 7.573193 & -1.061491 & 1.981685 \\
\hline $\mathrm{H}$ & 8.229745 & -0.350976 & 1.476765 \\
\hline $\mathrm{H}$ & 7.839491 & -1.154715 & 3.036945 \\
\hline $\mathrm{H}$ & 7.688213 & -2.043582 & 1.510476 \\
\hline C & 6.139915 & -0.624399 & 1.811892 \\
\hline O & 5.729779 & 0.084803 & 0.920848 \\
\hline $\mathrm{C}$ & -2.391927 & -1.876621 & 4.137605 \\
\hline $\mathrm{C}$ & -3.110224 & -0.890397 & 3.189667 \\
\hline $\mathrm{C}$ & -2.490827 & 0.497949 & 3.358938 \\
\hline $\mathrm{C}$ & -0.986282 & 0.413564 & 3.344648 \\
\hline
\end{tabular}




$\begin{array}{lrrr}\mathrm{H} & -0.463430 & 1.343044 & 3.153120 \\ \mathrm{H} & 0.500757 & -0.397397 & 0.943298 \\ \mathrm{H} & -0.785090 & -4.112081 & 4.120831 \\ \mathrm{H} & 1.651904 & -4.219673 & 3.721398 \\ \mathrm{H} & 5.029550 & 1.164361 & -1.191021 \\ \mathrm{~F} & 4.507072 & -2.576969 & 0.170706 \\ \mathrm{O} & -0.272383 & 0.085074 & 0.599725 \\ \mathrm{H} & -2.828789 & 1.173760 & 2.562160 \\ \mathrm{C} & -3.052731 & -1.322191 & 1.706325 \\ \mathrm{H} & -3.447762 & -2.330136 & 1.547634 \\ \mathrm{H} & -3.654448 & -0.629385 & 1.113565 \\ \mathrm{H} & -2.026902 & -1.286627 & 1.338555 \\ \mathrm{C} & -4.562551 & -1.064387 & 3.616380 \\ \mathrm{C} & -4.724120 & -2.496368 & 4.170138 \\ \mathrm{C} & -3.305570 & -3.117905 & 4.169493 \\ \mathrm{H} & -5.458942 & -3.050748 & 3.576663 \\ \mathrm{H} & -5.136895 & -2.415835 & 5.183110 \\ \mathrm{H} & -3.124863 & -3.746167 & 5.047164 \\ \mathrm{H} & -3.137478 & -3.742570 & 3.285875 \\ \mathrm{O} & -5.443154 & -0.235444 & 3.533929 \\ \mathrm{H} & -2.840180 & 0.951875 & 4.301445 \\ \mathrm{H} & -2.452016 & -1.418914 & 5.142483\end{array}$

\section{Structure: ${ }^{5}$ INT3b}

$\begin{array}{lrrr}\mathrm{C} & -3.171120 & 0.345821 & -1.551105 \\ \mathrm{C} & -4.266229 & -0.576422 & -1.739999 \\ \mathrm{C} & -3.752385 & -1.830468 & -1.637881 \\ \mathrm{C} & -2.329110 & -1.689023 & -1.441609 \\ \mathrm{~N} & -2.002325 & -0.358699 & -1.422409 \\ \mathrm{H} & -5.296986 & -0.292623 & -1.893980 \\ \mathrm{H} & -4.283580 & -2.768285 & -1.702250 \\ \mathrm{C} & 1.970565 & -1.725574 & -1.578939 \\ \mathrm{C} & 2.113291 & -3.156105 & -1.680327 \\ \mathrm{C} & 0.881327 & -3.699215 & -1.478804 \\ \mathrm{C} & -0.042366 & -2.604930 & -1.322737 \\ \mathrm{~N} & 0.647052 & -1.417694 & -1.367316 \\ \mathrm{H} & 3.040876 & -3.676304 & -1.866783 \\ \mathrm{H} & 0.605412 & -4.743995 & -1.486729 \\ \mathrm{C} & -0.303968 & 3.305598 & -0.312324 \\ \mathrm{C} & -1.256793 & 4.356504 & -0.046858 \\ \mathrm{C} & -2.469311 & 3.913666 & -0.473936 \\ \mathrm{C} & -2.281413 & 2.568309 & -0.961570 \\ \mathrm{~N} & -0.956537 & 2.232333 & -0.866359\end{array}$




\begin{tabular}{|c|c|c|c|}
\hline $\mathrm{H}$ & -1.022732 & 5.313991 & 0.393968 \\
\hline $\mathrm{H}$ & -3.416425 & 4.432079 & -0.436983 \\
\hline $\mathrm{C}$ & 1.991174 & 2.417984 & -0.496490 \\
\hline $\mathrm{C}$ & 3.421141 & 2.603809 & -0.542598 \\
\hline $\mathrm{C}$ & 3.960302 & 1.459108 & -1.037825 \\
\hline C & 2.870143 & 0.541219 & -1.260368 \\
\hline $\mathrm{N}$ & 1.684446 & 1.157970 & -0.943105 \\
\hline $\mathrm{H}$ & 3.939776 & 3.505035 & -0.250290 \\
\hline $\mathrm{C}$ & -3.322443 & 1.717818 & -1.340499 \\
\hline $\mathrm{C}$ & 3.023471 & -0.806210 & -1.595365 \\
\hline $\mathrm{C}$ & -1.431016 & -2.749394 & -1.297014 \\
\hline $\mathrm{C}$ & 1.076484 & 3.403270 & -0.112720 \\
\hline Mn & -0.159255 & 0.410562 & -1.154802 \\
\hline $\mathrm{Cl}$ & -0.017823 & 0.774724 & -3.435666 \\
\hline $\mathrm{C}$ & 1.630032 & 4.638332 & 0.507305 \\
\hline $\mathrm{C}$ & 2.281151 & 4.583264 & 1.744843 \\
\hline $\mathrm{C}$ & 1.556069 & 5.887701 & -0.117934 \\
\hline $\mathrm{C}$ & 2.828367 & 5.713890 & 2.344586 \\
\hline $\mathrm{C}$ & 2.088650 & 7.035421 & 0.465527 \\
\hline $\mathrm{C}$ & 2.728552 & 6.946316 & 1.700540 \\
\hline $\mathrm{C}$ & -4.703301 & 2.278091 & -1.349544 \\
\hline $\mathrm{C}$ & -5.148797 & 3.108097 & -2.380922 \\
\hline $\mathrm{C}$ & -5.590019 & 2.008965 & -0.301156 \\
\hline $\mathrm{C}$ & -6.434873 & 3.645149 & -2.381370 \\
\hline $\mathrm{C}$ & -6.881172 & 2.530830 & -0.282804 \\
\hline $\mathrm{C}$ & -7.300815 & 3.352512 & -1.329013 \\
\hline $\mathrm{C}$ & -1.984688 & -4.112135 & -1.060361 \\
\hline $\mathrm{C}$ & -2.621838 & -4.866270 & -2.048136 \\
\hline $\mathrm{C}$ & -1.910288 & -4.667187 & 0.221163 \\
\hline $\mathrm{C}$ & -3.180538 & -6.113532 & -1.771638 \\
\hline $\mathrm{C}$ & -2.472276 & -5.902388 & 0.52571 \\
\hline $\mathrm{C}$ & -3.106760 & -6.631416 & -0.478713 \\
\hline $\mathrm{C}$ & 4.398403 & -1.291407 & -1.896498 \\
\hline C & 5.068963 & -0.865762 & -3.048397 \\
\hline $\mathrm{C}$ & 5.093221 & -2.139810 & -1.031921 \\
\hline $\mathrm{C}$ & 6.374054 & -1.264052 & -3.331284 \\
\hline $\mathrm{C}$ & 6.392648 & -2.556489 & -1.29822 \\
\hline C & 7.039143 & -2.113402 & -2.448946 \\
\hline $\mathrm{F}$ & 2.399174 & 3.410866 & 2.391603 \\
\hline $\mathrm{F}$ & 3.441714 & 5.626608 & 3.528842 \\
\hline $\mathrm{F}$ & 3.245284 & 8.038608 & 2.265587 \\
\hline$F$ & 1.999889 & 8.215622 & -0.154588 \\
\hline $\mathrm{F}$ & 0.958961 & 6.004378 & -1.310101 \\
\hline F & 4.459890 & -0.040773 & -3.905910 \\
\hline
\end{tabular}




\begin{tabular}{|c|c|c|c|}
\hline$F$ & 6.989191 & -0.840160 & -4.439424 \\
\hline $\mathrm{F}$ & 8.290242 & -2.501576 & -2.704950 \\
\hline $\mathrm{F}$ & 7.037584 & -3.357715 & -0.434024 \\
\hline $\mathrm{F}$ & -2.728095 & -4.382289 & -3.291854 \\
\hline F & -3.787785 & -6.814183 & -2.734216 \\
\hline$F$ & -3.647654 & -7.819576 & -0.203571 \\
\hline F & -2.417084 & -6.377735 & 1.775991 \\
\hline F & -1.306675 & -3.991125 & 1.208572 \\
\hline$F$ & -4.331712 & 3.405310 & -3.397289 \\
\hline F & -6.843205 & 4.433805 & -3.380351 \\
\hline F & -8.534578 & 3.861110 & -1.321509 \\
\hline F & -7.713674 & 2.259836 & 0.725509 \\
\hline F & -5.201875 & 1.233044 & 0.720701 \\
\hline $\mathrm{C}$ & 4.051558 & -1.075062 & 2.758518 \\
\hline C & 3.354187 & -2.217996 & 3.078072 \\
\hline C & 1.956158 & -2.175983 & 3.292003 \\
\hline $\mathrm{C}$ & 1.266654 & -0.924393 & 3.190739 \\
\hline $\mathrm{C}$ & 2.022516 & 0.212602 & 2.813232 \\
\hline C & 3.390580 & 0.157363 & 2.600805 \\
\hline $\mathrm{H}$ & 3.892430 & -3.155680 & 3.176173 \\
\hline C & 1.233580 & -3.361225 & 3.609551 \\
\hline $\mathrm{C}$ & -0.175158 & -0.880052 & 3.450847 \\
\hline $\mathrm{H}$ & 1.534567 & 1.169797 & 2.679439 \\
\hline $\mathrm{H}$ & 3.935905 & 1.047263 & 2.316511 \\
\hline C & -0.836080 & -2.106099 & 3.784819 \\
\hline $\mathrm{C}$ & -0.129161 & -3.311192 & 3.845262 \\
\hline O & 5.442306 & -1.216317 & 2.710127 \\
\hline $\mathrm{C}$ & 7.642509 & -1.060399 & 1.897423 \\
\hline $\mathrm{H}$ & 8.287190 & -0.331783 & 1.403081 \\
\hline $\mathrm{H}$ & 7.917564 & -1.173083 & 2.948489 \\
\hline $\mathrm{H}$ & 7.764960 & -2.030671 & 1.404078 \\
\hline C & 6.203071 & -0.636775 & 1.746567 \\
\hline O & 5.778070 & 0.081306 & 0.869448 \\
\hline $\mathrm{C}$ & -2.295190 & -2.005980 & 4.130152 \\
\hline C & -3.035152 & -1.009352 & 3.209580 \\
\hline $\mathrm{C}$ & -2.420780 & 0.379574 & 3.389837 \\
\hline $\mathrm{C}$ & -0.917077 & 0.304568 & 3.341086 \\
\hline $\mathrm{H}$ & -0.404010 & 1.240089 & 3.155488 \\
\hline $\mathrm{H}$ & 0.441481 & -0.371554 & 0.947276 \\
\hline $\mathrm{H}$ & -0.665541 & -4.225667 & 4.081564 \\
\hline $\mathrm{H}$ & 1.769851 & -4.304563 & 3.666169 \\
\hline $\mathrm{H}$ & 5.003814 & 1.223280 & -1.168161 \\
\hline F & 4.520943 & -2.562685 & 0.105665 \\
\hline O & -0.301306 & 0.167904 & 0.622195 \\
\hline
\end{tabular}




$\begin{array}{lrrr}\mathrm{H} & -2.779444 & 1.068247 & 2.613719 \\ \mathrm{C} & -3.003284 & -1.412569 & 1.717693 \\ \mathrm{H} & -3.393709 & -2.420306 & 1.547075 \\ \mathrm{H} & -3.620267 & -0.712513 & 1.148457 \\ \mathrm{H} & -1.984984 & -1.361116 & 1.332255 \\ \mathrm{C} & -4.478464 & -1.200645 & 3.659569 \\ \mathrm{C} & -4.621596 & -2.645394 & 4.184900 \\ \mathrm{C} & -3.198154 & -3.255077 & 4.153763 \\ \mathrm{H} & -5.358640 & -3.192943 & 3.587787 \\ \mathrm{H} & -5.023443 & -2.590330 & 5.203874 \\ \mathrm{H} & -3.000358 & -3.895814 & 5.018633 \\ \mathrm{H} & -3.036663 & -3.864417 & 3.258360 \\ \mathrm{O} & -5.364794 & -0.375202 & 3.611941 \\ \mathrm{H} & -2.750314 & 0.813453 & 4.348807 \\ \mathrm{H} & -2.347900 & -1.565515 & 5.143238\end{array}$

\section{Structure: ${ }^{5}$ TS3a}

$\begin{array}{lrrr}\mathrm{C} & -1.617484 & 2.215787 & -1.149678 \\ \mathrm{C} & -2.924804 & 2.181300 & -1.764287 \\ \mathrm{C} & -3.017313 & 1.002655 & -2.438355 \\ \mathrm{C} & -1.801065 & 0.270596 & -2.179227 \\ \mathrm{~N} & -0.952714 & 1.058005 & -1.439494 \\ \mathrm{H} & -3.655962 & 2.974988 & -1.715189 \\ \mathrm{H} & -3.846543 & 0.644522 & -3.030127 \\ \mathrm{C} & 1.416721 & -2.309650 & -0.986771 \\ \mathrm{C} & 0.741956 & -3.554355 & -1.267663 \\ \mathrm{C} & -0.422284 & -3.243451 & -1.897310 \\ \mathrm{C} & -0.501702 & -1.803714 & -1.959142 \\ \mathrm{~N} & 0.622995 & -1.265550 & -1.393630 \\ \mathrm{H} & 1.118987 & -4.536325 & -1.023289 \\ \mathrm{H} & -1.183758 & -3.921106 & -2.254672 \\ \mathrm{C} & 2.452013 & 3.203981 & -0.275720 \\ \mathrm{C} & 2.216126 & 4.572223 & 0.116502 \\ \mathrm{C} & 0.868398 & 4.737053 & 0.219850 \\ \mathrm{C} & 0.257485 & 3.497773 & -0.191911 \\ \mathrm{~N} & 1.243104 & 2.578981 & -0.466253 \\ \mathrm{H} & 2.985558 & 5.303822 & 0.313776 \\ \mathrm{H} & 0.332659 & 5.633163 & 0.495316 \\ \mathrm{C} & 3.867423 & 1.190939 & -0.407985 \\ \mathrm{C} & 5.113431 & 0.492311 & -0.210026 \\ \mathrm{C} & 4.825251 & -0.838030 & -0.168763 \\ \mathrm{C} & 3.405508 & -0.974187 & -0.383815 \\ \mathrm{~N} & 2.853713 & 0.273584 & -0.525165 \\ & & & \\ & & & \end{array}$




\begin{tabular}{|c|c|c|c|}
\hline $\mathrm{H}$ & 6.076434 & 0.966074 & -0.085996 \\
\hline $\mathrm{H}$ & 5.512831 & -1.660129 & -0.032403 \\
\hline C & -1.104633 & 3.311924 & -0.442677 \\
\hline $\mathrm{C}$ & 2.725095 & -2.192268 & -0.507454 \\
\hline C & -1.605796 & -1.087970 & -2.437972 \\
\hline $\mathrm{C}$ & 3.702324 & 2.578450 & -0.324549 \\
\hline Mn & 0.947820 & 0.658636 & -0.953785 \\
\hline $\mathrm{Cl}$ & 1.559456 & 1.097597 & -3.145362 \\
\hline $\mathrm{C}$ & 4.928774 & 3.411071 & -0.174791 \\
\hline C & 5.265217 & 4.020249 & 1.037482 \\
\hline C & 5.808005 & 3.585147 & -1.248759 \\
\hline C & 6.417886 & 4.788548 & 1.179985 \\
\hline C & 6.971127 & 4.343237 & -1.127869 \\
\hline C & 7.273765 & 4.948050 & 0.091070 \\
\hline $\mathrm{C}$ & -2.107002 & 4.308366 & 0.024931 \\
\hline $\mathrm{C}$ & -3.053865 & 3.911460 & 0.977662 \\
\hline $\mathrm{C}$ & -2.232396 & 5.598698 & -0.495506 \\
\hline C & -4.107522 & 4.731042 & 1.365144 \\
\hline C & -3.259010 & 6.453909 & -0.096409 \\
\hline $\mathrm{C}$ & -4.205319 & 6.014809 & 0.829561 \\
\hline C & -2.670493 & -1.851180 & -3.145093 \\
\hline C & -2.405969 & -2.460435 & -4.378079 \\
\hline $\mathrm{C}$ & -3.952968 & -2.025391 & -2.611365 \\
\hline C & -3.371946 & -3.199579 & -5.057349 \\
\hline C & -4.936455 & -2.753038 & -3.275886 \\
\hline $\mathrm{C}$ & -4.642977 & -3.343908 & -4.503598 \\
\hline $\mathrm{C}$ & 3.484202 & -3.436927 & -0.206082 \\
\hline $\mathrm{C}$ & 3.832772 & -4.343002 & -1.214113 \\
\hline $\mathrm{C}$ & 3.907722 & -3.728547 & 1.094947 \\
\hline $\mathrm{C}$ & 4.559279 & -5.500260 & -0.944693 \\
\hline $\mathrm{C}$ & 4.648891 & -4.871293 & 1.383337 \\
\hline C & 4.969582 & -5.762347 & 0.360772 \\
\hline $\mathrm{F}$ & 4.463254 & 3.873952 & 2.102619 \\
\hline $\mathrm{F}$ & 6.712274 & 5.363069 & 2.350568 \\
\hline $\mathrm{F}$ & 8.382670 & 5.678632 & 0.216857 \\
\hline $\mathrm{F}$ & 7.793566 & 4.495819 & -2.169436 \\
\hline $\mathrm{F}$ & 5.543665 & 3.009788 & -2.425749 \\
\hline $\mathrm{F}$ & 3.468410 & -4.107141 & -2.479711 \\
\hline $\mathrm{F}$ & 4.875158 & -6.349052 & -1.926640 \\
\hline $\mathrm{F}$ & 5.673867 & -6.861790 & 0.632313 \\
\hline $\mathrm{F}$ & 5.060887 & -5.127305 & 2.631454 \\
\hline $\mathrm{F}$ & 3.612083 & -2.885755 & 2.095859 \\
\hline F & -1.195167 & -2.342784 & -4.932418 \\
\hline $\mathrm{F}$ & -3.089799 & -3.766562 & -6.233538 \\
\hline
\end{tabular}




\begin{tabular}{|c|c|c|c|}
\hline $\mathrm{F}$ & -5.576142 & -4.046755 & -5.146731 \\
\hline F & -6.152259 & -2.895307 & -2.737990 \\
\hline $\mathrm{F}$ & -4.272666 & -1.484776 & -1.425739 \\
\hline $\mathrm{F}$ & -2.975359 & 2.687768 & 1.523498 \\
\hline $\mathrm{F}$ & -5.023376 & 4.290767 & 2.237655 \\
\hline F & -5.200945 & 6.820236 & 1.198940 \\
\hline $\mathrm{F}$ & -3.354868 & 7.682644 & -0.611874 \\
\hline $\mathrm{F}$ & -1.362395 & 6.038134 & -1.414306 \\
\hline O & 0.477067 & 0.287184 & 0.75731 \\
\hline $\mathrm{C}$ & 0.096037 & -3.660507 & 3.385518 \\
\hline C & -0.044153 & -2.636317 & 2.486310 \\
\hline C & -1.342962 & -2.147564 & 2.182822 \\
\hline $\mathrm{C}$ & -2.511162 & -2.714426 & 2.821284 \\
\hline $\mathrm{C}$ & -2.291331 & -3.795547 & 3.723078 \\
\hline $\mathrm{C}$ & -1.026496 & -4.258947 & 4.002445 \\
\hline $\mathrm{H}$ & 0.822215 & -2.179309 & 2.020850 \\
\hline $\mathrm{C}$ & -1.568984 & -1.095292 & 1.293942 \\
\hline C & -3.797953 & -2.156376 & 2.531107 \\
\hline $\mathrm{H}$ & -3.136002 & -4.268155 & 4.21258 \\
\hline $\mathrm{H}$ & -0.873175 & -5.070289 & 4.704891 \\
\hline C & -3.914495 & -1.097369 & 1.63030 \\
\hline C & -2.769467 & -0.536686 & 1.00676 \\
\hline O & 1.385299 & -4.137950 & 3.650506 \\
\hline $\mathrm{C}$ & 3.275979 & -4.516293 & 5.012479 \\
\hline $\mathrm{H}$ & 3.595783 & -4.537567 & 6.054694 \\
\hline $\mathrm{H}$ & 3.367938 & -5.508601 & 4.562756 \\
\hline $\mathrm{H}$ & 3.917283 & -3.845326 & 4.435074 \\
\hline $\mathrm{C}$ & 1.852538 & -4.029944 & 4.939042 \\
\hline 0 & 1.202618 & -3.590631 & 5.854871 \\
\hline $\mathrm{C}$ & -5.298548 & -0.535676 & 1.460408 \\
\hline C & -5.881674 & -0.219845 & 2.866872 \\
\hline C & -5.564791 & 0.732895 & 0.62855 \\
\hline $\mathrm{H}$ & -5.555765 & 0.520450 & -0.44384 \\
\hline C & -6.944170 & 1.216685 & 1.14065 \\
\hline $\mathrm{H}$ & -7.767772 & 0.953091 & 0.46758 \\
\hline C & -7.176570 & 0.494063 & 2.48337 \\
\hline O & -8.217343 & 0.485124 & 3.10427 \\
\hline C & -5.020376 & 0.820130 & 3.62988 \\
\hline $\mathrm{H}$ & -5.513771 & 1.070551 & 4.57520 \\
\hline $\mathrm{H}$ & -4.035070 & 0.399394 & 3.85305 \\
\hline $\mathrm{H}$ & -4.869903 & 1.749031 & 3.071472 \\
\hline $\mathrm{H}$ & -6.993375 & 2.301051 & 1.29448 \\
\hline $\mathrm{H}$ & -4.797859 & 1.485829 & 0.81582 \\
\hline C & -6.017641 & -1.514762 & 3.69003 \\
\hline
\end{tabular}


$\begin{array}{ll}-5.094139 & -2.653359\end{array}$

3.151004

$-5.647922-3.209248$

2.379987

$\begin{array}{ll}-4.913604 & -3.367936\end{array}$

3. 956889

$-2.871919$

0.303509

0.333423

0.988841

0.818308

1.395554

$-5.936306-1.328053$

1.031751

$-5.767041$

$-1.312240$

4.736389

$-7.058409$

$-1.857347$

3.682447

\section{Structure: ${ }^{5} \mathrm{TS} 3 \mathrm{~b}$}

\begin{tabular}{|c|c|c|c|}
\hline $\mathrm{C}$ & -3.220332 & 0.233689 & -1.549792 \\
\hline C & -4.287261 & -0.723354 & -1.732224 \\
\hline C & -3.730551 & -1.960291 & -1.649380 \\
\hline C & -2.309717 & -1.772484 & -1.469066 \\
\hline $\mathrm{N}$ & -2.026807 & -0.432800 & -1.44711 \\
\hline $\mathrm{H}$ & -5.328856 & -0.472957 & -1.87098 \\
\hline $\mathrm{H}$ & -4.231571 & -2.914143 & -1.717891 \\
\hline C & 1.993821 & -1.668230 & -1.586501 \\
\hline C & 2.179998 & -3.091868 & -1.713780 \\
\hline $\mathrm{C}$ & 0.965408 & -3.675898 & -1.521192 \\
\hline $\mathrm{C}$ & 0.008613 & -2.612669 & -1.348608 \\
\hline $\mathrm{N}$ & 0.660845 & -1.404322 & -1.370061 \\
\hline $\mathrm{H}$ & 3.122232 & -3.580832 & -1.91094 \\
\hline $\mathrm{H}$ & 0.721819 & -4.728447 & -1.548378 \\
\hline C & -0.444028 & 3.303105 & -0.351771 \\
\hline C & -1.427736 & 4.328210 & -0.095278 \\
\hline C & -2.630743 & 3.836162 & -0.49557 \\
\hline $\mathrm{C}$ & -2.402413 & 2.490207 & -0.96523 \\
\hline $\mathrm{N}$ & -1.066274 & 2.201664 & -0.881765 \\
\hline $\mathrm{H}$ & -1.220589 & 5.301080 & 0.325266 \\
\hline $\mathrm{H}$ & -3.593923 & 4.323797 & -0.45438 \\
\hline C & 1.881851 & 2.477832 & -0.50003 \\
\hline $\mathrm{C}$ & 3.309364 & 2.694762 & -0.49650 \\
\hline $\mathrm{C}$ & 3.889964 & 1.561232 & -0.971746 \\
\hline C & 2.825430 & 0.622220 & -1.235011 \\
\hline $\mathrm{N}$ & 1.618584 & 1.211371 & -0.95519 \\
\hline $\mathrm{H}$ & 3.798300 & 3.607092 & -0.18724 \\
\hline $\mathrm{C}$ & -3.416483 & 1.598269 & -1.32546 \\
\hline C & 3.019113 & -0.717984 & -1.58355 \\
\hline $\mathrm{C}$ & -1.375329 & -2.802514 & -1.32190 \\
\hline $\mathrm{C}$ & 0.932534 & 3.440926 & -0.14443 \\
\hline $\mathrm{Mn}$ & -0.207235 & 0.405745 & -1.18257 \\
\hline $\mathrm{Cl}$ & -0.072928 & 0.777537 & -3.4924 \\
\hline
\end{tabular}




\begin{tabular}{|c|c|c|c|}
\hline C & 1.438076 & 4.700853 & 0.467611 \\
\hline C & 2.037812 & 4.689126 & 1.731299 \\
\hline C & 1.358028 & 5.933125 & -0.189226 \\
\hline C & 2.534345 & 5.845950 & 2.325316 \\
\hline C & 1.840502 & 7.106361 & 0.387189 \\
\hline $\mathrm{C}$ & 2.432414 & 7.060415 & 1.648277 \\
\hline $\mathrm{C}$ & -4.819026 & 2.101612 & -1.291941 \\
\hline $\mathrm{C}$ & -5.333346 & 2.914165 & -2.304575 \\
\hline $\mathrm{C}$ & -5.658109 & 1.792621 & -0.215505 \\
\hline $\mathrm{C}$ & -6.640272 & 3.396550 & -2.259786 \\
\hline C & -6.968798 & 2.258997 & -0.152046 \\
\hline C & -7.457558 & 3.064757 & -1.180549 \\
\hline $\mathrm{C}$ & -1.877958 & -4.179030 & -1.054537 \\
\hline $\mathrm{C}$ & -2.529521 & -4.961915 & -2.010271 \\
\hline C & -1.739152 & -4.721214 & 0.228497 \\
\hline C & -3.042048 & -6.221571 & -1.702546 \\
\hline $\mathrm{C}$ & -2.254765 & -5.968597 & 0.563907 \\
\hline $\mathrm{C}$ & -2.905571 & -6.725017 & -0.409305 \\
\hline $\mathrm{C}$ & 4.408427 & -1.158133 & -1.886166 \\
\hline $\mathrm{C}$ & 5.075754 & -0.683559 & -3.021289 \\
\hline C & 5.120570 & -2.009724 & -1.039939 \\
\hline $\mathrm{C}$ & 6.393303 & -1.038437 & -3.303841 \\
\hline $\mathrm{C}$ & 6.433369 & -2.381158 & -1.305007 \\
\hline C & 7.075852 & -1.891082 & -2.438086 \\
\hline $\mathrm{F}$ & 2.152230 & 3.534640 & 2.410766 \\
\hline $\mathrm{F}$ & 3.100199 & 5.801276 & 3.535694 \\
\hline $\mathrm{F}$ & 2.900707 & 8.177473 & 2.207620 \\
\hline F & 1.748076 & 8.270235 & -0.262683 \\
\hline $\mathrm{F}$ & 0.801672 & 6.007095 & -1.403824 \\
\hline $\mathrm{F}$ & 4.450777 & 0.145809 & -3.862576 \\
\hline F & 7.004632 & -0.569559 & -4.396054 \\
\hline $\mathrm{F}$ & 8.340468 & -2.235265 & -2.692134 \\
\hline $\mathrm{F}$ & 7.095416 & -3.178883 & -0.449911 \\
\hline $\mathrm{F}$ & -2.696814 & -4.494333 & -3.253617 \\
\hline $\mathrm{F}$ & -3.664434 & -6.947966 & -2.635902 \\
\hline $\mathrm{F}$ & -3.401980 & -7.925220 & -0.103691 \\
\hline $\mathrm{F}$ & -2.138784 & -6.431223 & 1.815101 \\
\hline $\mathrm{F}$ & -1.116615 & -4.023385 & 1.190642 \\
\hline $\mathrm{F}$ & -4.564151 & 3.248739 & -3.346266 \\
\hline $\mathrm{F}$ & -7.114455 & 4.169892 & -3.241696 \\
\hline $\mathrm{F}$ & -8.711191 & 3.520145 & -1.130186 \\
\hline F & -7.755435 & 1.950145 & 0.882081 \\
\hline F & -5.202950 & 1.031371 & 0.790062 \\
\hline $\mathrm{C}$ & 4.217126 & -1.009133 & 2.713164 \\
\hline
\end{tabular}




\begin{tabular}{|c|c|c|c|}
\hline$C$ & 3.537264 & -2.143080 & 3.095857 \\
\hline C & 2.135950 & -2.112052 & 3.290339 \\
\hline $\mathrm{C}$ & 1.425569 & -0.883734 & 3.100381 \\
\hline C & 2.163824 & 0.243213 & 2.663600 \\
\hline C & 3.534516 & 0.199600 & 2.476201 \\
\hline $\mathrm{H}$ & 4.089922 & -3.063699 & 3.255407 \\
\hline $\mathrm{C}$ & 1.427007 & -3.286382 & 3.671692 \\
\hline C & -0.017390 & -0.853804 & 3.336128 \\
\hline $\mathrm{H}$ & 1.660277 & 1.179040 & 2.458950 \\
\hline $\mathrm{H}$ & 4.066791 & 1.080287 & 2.144128 \\
\hline C & -0.663061 & -2.057140 & 3.751984 \\
\hline $\mathrm{C}$ & 0.062932 & -3.246548 & 3.893984 \\
\hline O & 5.608898 & -1.130134 & 2.676154 \\
\hline C & 7.817129 & -0.948863 & 1.886834 \\
\hline $\mathrm{H}$ & 8.454402 & -0.204762 & 1.406003 \\
\hline $\mathrm{H}$ & 8.075175 & -1.058721 & 2.942338 \\
\hline $\mathrm{H}$ & 7.970025 & -1.913596 & 1.391040 \\
\hline C & 6.372115 & -0.556768 & 1.708611 \\
\hline 0 & 5.945426 & 0.133984 & 0.810592 \\
\hline $\mathrm{C}$ & -2.120311 & -1.953969 & 4.104690 \\
\hline C & -2.890069 & -1.033709 & 3.131364 \\
\hline $\mathrm{C}$ & -2.281542 & 0.368103 & 3.182130 \\
\hline $\mathrm{C}$ & -0.780791 & 0.307668 & 3.105853 \\
\hline $\mathrm{H}$ & -0.280589 & 1.248698 & 2.919632 \\
\hline $\mathrm{H}$ & 0.338255 & -0.472361 & 0.898719 \\
\hline $\mathrm{H}$ & -0.459904 & -4.152078 & 4.187525 \\
\hline $\mathrm{H}$ & 1.977198 & -4.215928 & 3.790506 \\
\hline $\mathrm{H}$ & 4.942195 & 1.346491 & -1.066423 \\
\hline $\mathrm{F}$ & 4.554939 & -2.474298 & 0.08486 \\
\hline O & -0.347878 & 0.169645 & 0.645989 \\
\hline $\mathrm{H}$ & -2.664053 & 0.995622 & 2.367836 \\
\hline C & -2.905894 & -1.553209 & 1.677303 \\
\hline $\mathrm{H}$ & -3.305676 & -2.568521 & 1.598868 \\
\hline $\mathrm{H}$ & -3.537768 & -0.895575 & 1.076056 \\
\hline $\mathrm{H}$ & -1.901456 & -1.537238 & 1.257525 \\
\hline $\mathrm{C}$ & -4.317169 & -1.198390 & 3.640403 \\
\hline C & -4.435789 & -2.612242 & 4.250291 \\
\hline C & -3.005761 & -3.209028 & 4.228967 \\
\hline $\mathrm{H}$ & -5.175027 & -3.200510 & 3.696250 \\
\hline $\mathrm{H}$ & -4.822543 & -2.504524 & 5.270617 \\
\hline $\mathrm{H}$ & -2.783707 & -3.789446 & 5.129721 \\
\hline $\mathrm{H}$ & -2.853724 & -3.872844 & 3.370941 \\
\hline O & -5.205751 & -0.376920 & 3.579165 \\
\hline $\mathrm{H}$ & -2.581398 & 0.872865 & 4.11619 \\
\hline
\end{tabular}




\section{Structure: ${ }^{1}$ TS4}

\begin{tabular}{|c|c|c|c|}
\hline $\mathrm{C}$ & 2.439997 & 2.382705 & -0.753493 \\
\hline $\mathrm{C}$ & 2.709697 & 3.757513 & -0.407564 \\
\hline C & 1.508580 & 4.357487 & -0.190302 \\
\hline C & 0.490370 & 3.367208 & -0.450322 \\
\hline $\mathrm{N}$ & 1.081596 & 2.168128 & -0.771075 \\
\hline $\mathrm{H}$ & 3.693822 & 4.197590 & -0.332223 \\
\hline $\mathrm{H}$ & 1.325190 & 5.382989 & 0.094847 \\
\hline C & -2.833896 & 0.878266 & -1.616088 \\
\hline $\mathrm{C}$ & -3.863835 & 1.881457 & -1.463339 \\
\hline $\mathrm{C}$ & -3.264171 & 2.997162 & -0.968972 \\
\hline $\mathrm{C}$ & -1.855976 & 2.696599 & -0.846975 \\
\hline $\mathrm{N}$ & -1.622566 & 1.411255 & -1.254140 \\
\hline $\mathrm{H}$ & -4.907763 & 1.745703 & -1.706216 \\
\hline $\mathrm{H}$ & -3.721147 & 3.948603 & -0.737738 \\
\hline C & 2.095624 & -1.875287 & -1.135538 \\
\hline C & 3.498028 & -2.176490 & -0.949623 \\
\hline C & 4.160108 & -0.990858 & -0.976780 \\
\hline $\mathrm{C}$ & 3.161275 & 0.047144 & -1.103430 \\
\hline $\mathrm{N}$ & 1.923820 & -0.517796 & -1.225759 \\
\hline $\mathrm{H}$ & 3.921725 & -3.158656 & -0.807692 \\
\hline $\mathrm{H}$ & 5.222520 & -0.825700 & -0.873550 \\
\hline $\mathrm{C}$ & -0.243586 & -2.542641 & -1.587054 \\
\hline $\mathrm{C}$ & -1.253992 & -3.542331 & -1.860963 \\
\hline $\mathrm{C}$ & -2.404216 & -2.873158 & -2.135059 \\
\hline $\mathrm{C}$ & -2.109644 & -1.461262 & -1.993424 \\
\hline $\mathrm{N}$ & -0.784571 & -1.295249 & -1.708109 \\
\hline $\mathrm{H}$ & -1.087788 & -4.610321 & -1.861889 \\
\hline $\mathrm{H}$ & -3.370172 & -3.288769 & -2.383556 \\
\hline C & 3.424949 & 1.411035 & -0.942335 \\
\hline C & -3.076057 & -0.449272 & -1.985088 \\
\hline C & -0.885720 & 3.610924 & -0.421350 \\
\hline $\mathrm{C}$ & 1.081901 & -2.830627 & -1.243199 \\
\hline Mn & 0.143269 & 0.444085 & -1.205399 \\
\hline $\mathrm{Cl}$ & 0.391599 & 0.877446 & -3.461902 \\
\hline $\mathrm{C}$ & 1.403363 & -4.236848 & -0.871549 \\
\hline C & 0.897958 & -4.754205 & 0.325541 \\
\hline $\mathrm{C}$ & 2.220822 & -5.074384 & -1.632775 \\
\hline C & 1.198185 & -6.038339 & 0.764855 \\
\hline C & 2.535484 & -6.367565 & -1.216775 \\
\hline $\mathrm{C}$ & 2.021772 & -6.849975 & -0.013857 \\
\hline
\end{tabular}


C

C

C

C

C

C

C

C

C

C

C

C

C

C

C

C

C

C

F

F

F

$\mathrm{F}$

F

F

F

F

$\mathrm{F}$

F

F

F

F

F

F

F

F

F

F

F

O

C

C

C

C

C
4.860006

5.462059

5.683649

6.825737

7.050602

7.624286

$-1.363851$

$-1.198803$

$-2.019547$

$-1.658667$

$-2.495259$

$-2.311393$

$-4.492178$

$-4.968211$

$-5.397181$

$-6.289985$

$-6.721108$

$-7.168920$

0.101248

0.706615

2.319228

3. 323021

2.738842

$-4.147911$

$-6.721646$

$-8.434843$

$-7.552675$

$-4.990534$

$-0.576211$

$-1.484576$

$-2.758567$

$-3.117903$

$-2.207096$

4.730508

7.376457

8. 934542

7.815039

5.166522

$-0.123391$

3. 223923

2. 341242

0.966900

0.454447

1. 394802
$1.803747-0.843577$

$2.078502 \quad 0.386255$

$1.811270-1.973967$

$2.328454 \quad 0.501358$

$2.072656-1.885916$

$2.324410-0.640407$

$\begin{array}{ll}4.927746 & 0.086478\end{array}$

$6.113698-0.634307$

$5.014114 \quad 1.318182$

$7.337318-0.150563$

$6.221962 \quad 1.820279$

$7.388999 \quad 1.079997$

$-0.854136-2.218896$

$-1.215361-3.481937$

$-0.918751-1.156831$

$-1.615595-3.678932$

$-1.307810-1.324442$

$-1.659960-2.597051$

$\begin{array}{ll}-3.993161 & 1.094873\end{array}$

$\begin{array}{ll}-6.490108 & 1.925713\end{array}$

$\begin{array}{ll}-8.085973 & 0.392404\end{array}$

$-7.147984-1.963419$

$-4.633969-2.785774$

$-1.188669-4.536582$

$-1.953864-4.897353$

$-2.039296-2.779460$

$-1.351757-0.276108$

$\begin{array}{ll}-0.595213 & 0.085078\end{array}$

$6.093105-1.818329$

$8.456873-0.859355$

$8.554687 \quad 1.551071$

$6.271687 \quad 3.002789$

$3.905254 \quad 2.056759$

$2.078551 \quad 1.512454$

$2.526199 \quad 1.707311$

$2.557550-0.540155$

$2.071593-2.981643$

$1.543842-3.175721$

$\begin{array}{ll}-0.081289 & 0.351258\end{array}$

$-1.009412 \quad 2.683185$

$\begin{array}{ll}-0.001432 & 2.330968\end{array}$

$-0.190406 \quad 2.500056$

$\begin{array}{ll}-1.408519 & 3.032061\end{array}$

$-2.404300 \quad 3.396877$ 


\begin{tabular}{|c|c|c|c|}
\hline C & 2.757367 & -2.219468 & 3.226190 \\
\hline $\mathrm{H}$ & 2.723923 & 0.913331 & 1.894906 \\
\hline C & 0.029075 & 0.779076 & 1.964149 \\
\hline $\mathrm{C}$ & -0.973168 & -1.593138 & 3.138113 \\
\hline $\mathrm{H}$ & 1.053372 & -3.349660 & 3.801556 \\
\hline $\mathrm{H}$ & 3.457414 & -3.000164 & 3.491012 \\
\hline C & -1.847129 & -0.534566 & 2.787265 \\
\hline C & -1.347938 & 0.669890 & 2.308665 \\
\hline 0 & 4.569804 & -0.703665 & 2.523215 \\
\hline C & 6.834382 & -0.971767 & 1.915807 \\
\hline $\mathrm{H}$ & 6.837070 & 0.068794 & 2.241931 \\
\hline $\mathrm{H}$ & 7.560676 & -1.548831 & 2.496297 \\
\hline $\mathrm{H}$ & 7.141530 & -1.030479 & 0.866203 \\
\hline C & 5.474898 & -1.616341 & 2.052599 \\
\hline O & 5.214958 & -2.762416 & 1.776908 \\
\hline C & -3.329367 & -0.793612 & 2.816862 \\
\hline $\mathrm{C}$ & -3.738694 & -1.765395 & 3.937114 \\
\hline $\mathrm{C}$ & -4.352284 & 0.355663 & 2.890223 \\
\hline $\mathrm{H}$ & -4.390157 & 0.938632 & 1.966243 \\
\hline $\mathrm{C}$ & -5.672289 & -0.408099 & 3.158243 \\
\hline $\mathrm{H}$ & -6.195852 & -0.633821 & 2.221807 \\
\hline $\mathrm{C}$ & -5.263640 & -1.751686 & 3.794133 \\
\hline O & -6.017531 & -2.641699 & 4.121108 \\
\hline C & -3.432474 & -1.226804 & 5.356086 \\
\hline $\mathrm{H}$ & -3.740416 & -1.967447 & 6.101442 \\
\hline $\mathrm{H}$ & -2.364366 & -1.023582 & 5.483689 \\
\hline $\mathrm{H}$ & -3.969235 & -0.296812 & 5.570756 \\
\hline $\mathrm{H}$ & -6.382225 & 0.119995 & 3.802843 \\
\hline $\mathrm{H}$ & -4.111447 & 1.044222 & 3.707798 \\
\hline $\mathrm{C}$ & -3.038280 & -3.096938 & 3.682376 \\
\hline $\mathrm{C}$ & -1.502056 & -2.911250 & 3.684269 \\
\hline $\mathrm{H}$ & -3.360993 & -3.483683 & 2.707858 \\
\hline $\mathrm{H}$ & -3.324016 & -3.846503 & 4.427833 \\
\hline $\mathrm{H}$ & -1.043490 & -3.737139 & 3.130075 \\
\hline $\mathrm{H}$ & -1.126951 & -3.008766 & 4.713860 \\
\hline $\mathrm{H}$ & -2.018289 & 1.471993 & 2.023477 \\
\hline $\mathrm{H}$ & 0.416664 & 1.746535 & 1.665822 \\
\hline $\mathrm{H}$ & -3.545591 & -1.314796 & 1.869571 \\
\hline
\end{tabular}

\section{Structure: ${ }^{3}$ TS4}

$\begin{array}{llll}\text { C } & 2.580677 & 2.094422 & -0.979056 \\ \mathrm{C} & 2.969195 & 3.461625 & -0.732186 \\ \mathrm{C} & 1.829371 & 4.166962 & -0.498240\end{array}$




\begin{tabular}{|c|c|c|c|}
\hline $\mathrm{C}$ & 0.727608 & 3.249872 & -0.653177 \\
\hline $\mathrm{N}$ & 1.209168 & 1.994808 & -0.935803 \\
\hline $\mathrm{H}$ & 3.985828 & 3.826907 & -0.728290 \\
\hline $\mathrm{H}$ & 1.731892 & 5.220184 & -0.276693 \\
\hline C & -2.838806 & 1.048915 & -1.639229 \\
\hline C & -3.774659 & 2.137324 & -1.455584 \\
\hline C & -3.063286 & 3.203758 & -1.004317 \\
\hline C & -1.680030 & 2.789563 & -0.949644 \\
\hline $\mathrm{N}$ & -1.573169 & 1.486544 & -1.351290 \\
\hline $\mathrm{H}$ & -4.836915 & 2.082524 & -1.644488 \\
\hline $\mathrm{H}$ & -3.427458 & 4.190133 & -0.754835 \\
\hline C & 1.833664 & -2.124644 & -1.126489 \\
\hline C & 3.205363 & -2.549245 & -0.957319 \\
\hline C & 3.975893 & -1.433199 & -1.024949 \\
\hline C & 3.077980 & -0.310616 & -1.180939 \\
\hline $\mathrm{N}$ & 1.789385 & -0.760837 & -1.253631 \\
\hline $\mathrm{H}$ & 3.530476 & -3.570302 & -0.823011 \\
\hline $\mathrm{H}$ & 5.049040 & -1.360746 & -0.930435 \\
\hline C & -0.558059 & -2.589342 & -1.546471 \\
\hline $\mathrm{C}$ & -1.659848 & -3.498191 & -1.774285 \\
\hline C & -2.751854 & -2.736422 & -2.043807 \\
\hline $\mathrm{C}$ & -2.332977 & -1.353500 & -1.952145 \\
\hline $\mathrm{N}$ & -0.989905 & -1.302087 & -1.699986 \\
\hline $\mathrm{H}$ & -1.590087 & -4.576267 & -1.747717 \\
\hline $\mathrm{H}$ & -3.756301 & -3.069892 & -2.260660 \\
\hline C & 3.472946 & 1.028513 & -1.118167 \\
\hline C & -3.208615 & -0.263780 & -1.948004 \\
\hline C & -0.617679 & 3.616248 & -0.581372 \\
\hline $\mathrm{C}$ & 0.740044 & -2.987475 & -1.214539 \\
\hline Mn & 0.101426 & 0.356572 & -1.285301 \\
\hline $\mathrm{Cl}$ & 0.377630 & 0.657830 & -3.595620 \\
\hline $\mathrm{C}$ & 0.950356 & -4.422310 & -0.872381 \\
\hline $\mathrm{C}$ & 0.463231 & -4.918413 & 0.340481 \\
\hline C & 1.633445 & -5.314266 & -1.701710 \\
\hline $\mathrm{C}$ & 0.645685 & -6.242599 & 0.725431 \\
\hline C & 1.831094 & -6.646080 & -1.339149 \\
\hline C & 1.333544 & -7.110211 & -0.122200 \\
\hline C & 4.936772 & 1.304359 & -1.097133 \\
\hline C & 5.599170 & 1.726164 & 0.058681 \\
\hline $\mathrm{C}$ & 5.719876 & 1.088040 & -2.236955 \\
\hline C & 6.975128 & 1.929704 & 0.086983 \\
\hline $\mathrm{C}$ & 7.100174 & 1.283776 & -2.231445 \\
\hline C & 7.730924 & 1.702315 & -1.060977 \\
\hline $\mathrm{C}$ & -0.937436 & 4.972250 & -0.05261 \\
\hline
\end{tabular}




$\begin{array}{rrr}-1.207150 & 6.060239 & -0.884727 \\ -0.980326 & 5.185778 & 1.327090 \\ -1.505742 & 7.319470 & -0.365482 \\ -1.275358 & 6.432521 & 1.870886 \\ -1.537736 & 7.503059 & 1.016236 \\ -4.665417 & -0.555474 & -2.079166 \\ -5.260373 & -0.897363 & -3.295507 \\ -5.490322 & -0.525436 & -0.951740 \\ -6.621610 & -1.189694 & -3.386096 \\ -6.850287 & -0.805868 & -1.013801 \\ -7.418365 & -1.141500 & -2.242464 \\ -0.201229 & -4.100172 & 1.172551 \\ 0.172593 & -6.681423 & 1.897590 \\ 1.518150 & -8.383253 & 0.232605 \\ 2.490211 & -7.480115 & -2.149225 \\ 2.129731 & -4.892060 & -2.870407 \\ -4.520157 & -0.958533 & -4.406422 \\ -7.168414 & -1.513122 & -4.561316 \\ -8.720895 & -1.416808 & -2.324285 \\ -7.603131 & -0.761574 & 0.092371 \\ -4.965539 & -0.212921 & 0.247440 \\ -1.184733 & 5.904993 & -2.212502 \\ -1.759001 & 8.348372 & -1.179496 \\ -1.822589 & 8.703974 & 1.523097 \\ -1.312800 & 6.610065 & 3.195297 \\ -0.739278 & 4.162843 & 2.166941 \\ 4.915914 & 1.939084 & 1.195163 \\ 7.578683 & 2.304844 & 1.224772 \\ 9.052395 & 1.884381 & -1.038134 \\ 7.821116 & 1.068393 & -3.335140 \\ 5.146339 & 0.670777 & -3.368345 \\ -0.224688 & -0.072681 & 0.325862 \\ 3.280901 & -1.026069 & 2.514978 \\ 2.437741 & 0.025877 & 2.210739 \\ 1.062001 & -0.092579 & 2.446428 \\ 0.517584 & -1.281562 & 3.015022 \\ 1.422649 & -2.329013 & 3.323639 \\ -179717 & -2.215903 & 3.073887 \\ -1.84669 & 0.922052 & 1.756745 \\ -1.969 & -1.388297 & 3.201300 \\ -1.254786 & -3.749790 \\ -1.026843 & 3.311348 \\ -1.294086 & 2.881873\end{array}$




$\begin{array}{lrrr}\mathrm{C} & -1.200772 & 0.881539 & 2.367369 \\ \mathrm{O} & 4.614787 & -0.863118 & 2.154079 \\ \mathrm{C} & 6.952201 & -0.818555 & 2.407365 \\ \mathrm{H} & 7.044224 & 0.271809 & 2.379198 \\ \mathrm{H} & 7.761860 & -1.237008 & 3.005907 \\ \mathrm{H} & 7.012811 & -1.181097 & 1.376566 \\ \mathrm{C} & 5.627988 & -1.194989 & 3.021437 \\ \mathrm{O} & 5.456692 & -1.690196 & 4.106552 \\ \mathrm{C} & -3.234178 & -0.481887 & 2.959790 \\ \mathrm{C} & -3.653871 & -1.422984 & 4.102109 \\ \mathrm{C} & -4.201761 & 0.712871 & 3.051709 \\ \mathrm{H} & -4.241138 & 1.288122 & 2.122728 \\ \mathrm{C} & -5.546625 & 0.012130 & 3.364533 \\ \mathrm{H} & -6.107962 & -0.195956 & 2.445990 \\ \mathrm{C} & -5.179735 & -1.344071 & 4.000360 \\ \mathrm{O} & -5.963682 & -2.197642 & 4.353124 \\ \mathrm{C} & -3.283355 & -0.883618 & 5.505182 \\ \mathrm{H} & -3.599437 & -1.602873 & 6.267876 \\ \mathrm{H} & -2.204324 & -0.724735 & 5.597758 \\ \mathrm{H} & -3.773925 & 0.070412 & 5.724958 \\ \mathrm{H} & -6.212576 & 0.576442 & 4.025294 \\ \mathrm{H} & -3.907793 & 1.396958 & 3.855661 \\ \mathrm{C} & -3.019748 & -2.786505 & 3.843239 \\ \mathrm{C} & -1.477645 & -2.669204 & 3.796363 \\ \mathrm{H} & -3.387628 & -3.169584 & 2.883220 \\ \mathrm{H} & -3.315127 & -3.514834 & 4.605930 \\ \mathrm{H} & -1.076405 & -3.529596 & 3.251738 \\ \mathrm{H} & -1.075215 & -2.757361 & 4.816454 \\ \mathrm{H} & -1.842249 & 1.716072 & 2.107017 \\ \mathrm{H} & -366853 & 1.857613 & 1.611525 \\ \mathrm{H} & -505138 & -1.000116 & 2.024605\end{array}$

\section{Structure: ${ }^{5}$ TS4}

$\begin{array}{lrrr}\mathrm{C} & 2.490822 & 2.159057 & -0.912905 \\ \mathrm{C} & 2.825849 & 3.542042 & -0.669939 \\ \mathrm{C} & 1.653528 & 4.210233 & -0.494197 \\ \mathrm{C} & 0.591955 & 3.249467 & -0.679131 \\ \mathrm{~N} & 1.127170 & 2.012026 & -0.918671 \\ \mathrm{H} & 3.827894 & 3.943691 & -0.634820 \\ \mathrm{H} & 1.510820 & 5.263620 & -0.299688 \\ \mathrm{C} & -2.882337 & 0.903029 & -1.690469 \\ \mathrm{C} & -3.853595 & 1.973569 & -1.597152 \\ \mathrm{C} & -3.181335 & 3.083212 & -1.197729\end{array}$




\begin{tabular}{|c|c|c|c|}
\hline C & -1.787679 & 2.710378 & -1.084059 \\
\hline $\mathrm{N}$ & -1.636068 & 1.391685 & -1.417752 \\
\hline $\mathrm{H}$ & -4.909934 & 1.875919 & -1.801145 \\
\hline $\mathrm{H}$ & -3.579282 & 4.070523 & -1.012168 \\
\hline C & 1.917262 & -2.096573 & -1.054909 \\
\hline $\mathrm{C}$ & 3.306823 & -2.462265 & -0.893993 \\
\hline C & 4.025603 & -1.312575 & -0.951490 \\
\hline $\mathrm{C}$ & 3.078645 & -0.229490 & -1.101120 \\
\hline $\mathrm{N}$ & 1.808751 & -0.737640 & -1.177861 \\
\hline $\mathrm{H}$ & 3.675668 & -3.468860 & -0.762551 \\
\hline $\mathrm{H}$ & 5.094158 & -1.192995 & -0.854143 \\
\hline $\mathrm{C}$ & -0.463065 & -2.658470 & -1.392274 \\
\hline $\mathrm{C}$ & -1.538047 & -3.607676 & -1.551436 \\
\hline $\mathrm{C}$ & -2.664894 & -2.893852 & -1.814231 \\
\hline C & -2.296000 & -1.497815 & -1.798097 \\
\hline $\mathrm{N}$ & -0.945619 & -1.386280 & -1.569775 \\
\hline $\mathrm{H}$ & -1.432643 & -4.681148 & -1.487123 \\
\hline $\mathrm{H}$ & -3.661564 & -3.272518 & -1.987471 \\
\hline $\mathrm{C}$ & 3.422544 & 1.122688 & -1.033122 \\
\hline $\mathrm{C}$ & -3.208181 & -0.443428 & -1.884401 \\
\hline $\mathrm{C}$ & -0.767237 & 3.576675 & -0.692566 \\
\hline $\mathrm{C}$ & 0.860030 & -3.005974 & -1.114853 \\
\hline Mn & 0.076810 & 0.310613 & -1.280373 \\
\hline $\mathrm{Cl}$ & 0.423198 & 0.649922 & -3.573812 \\
\hline $\mathrm{C}$ & 1.156593 & -4.440656 & -0.841022 \\
\hline $\mathrm{C}$ & 0.768272 & -5.022461 & 0.368609 \\
\hline $\mathrm{C}$ & 1.833417 & -5.253058 & -1.754349 \\
\hline $\mathrm{C}$ & 1.035307 & -6.354664 & 0.669113 \\
\hline $\mathrm{C}$ & 2.117123 & -6.589494 & -1.476596 \\
\hline $\mathrm{C}$ & 1.713527 & -7.140816 & -0.261395 \\
\hline $\mathrm{C}$ & 4.875448 & 1.453215 & -1.016007 \\
\hline $\mathrm{C}$ & 5.523735 & 1.935548 & 0.123953 \\
\hline $\mathrm{C}$ & 5.667045 & 1.231333 & -2.149799 \\
\hline $\mathrm{C}$ & 6.892630 & 2.183147 & 0.145707 \\
\hline $\mathrm{C}$ & 7.039919 & 1.473023 & -2.151418 \\
\hline $\mathrm{C}$ & 7.656659 & 1.946154 & -0.994433 \\
\hline C & -1.147490 & 4.940813 & -0.227554 \\
\hline $\mathrm{C}$ & -1.478812 & 5.970604 & -1.110587 \\
\hline $\mathrm{C}$ & -1.182174 & 5.227329 & 1.139201 \\
\hline C & -1.831097 & 7.240053 & -0.653930 \\
\hline $\mathrm{C}$ & -1.527844 & 6.486371 & 1.621237 \\
\hline $\mathrm{C}$ & -1.852496 & 7.496599 & 0.716251 \\
\hline C & -4.654110 & -0.793790 & -1.987802 \\
\hline C & -5.232303 & -1.284243 & -3.160900 \\
\hline
\end{tabular}


C

F

F

\begin{tabular}{|c|c|c|}
\hline-5.486014 & -0.669585 & -0.871733 \\
\hline-6.582116 & -1.631557 & -3.220963 \\
\hline-6.835032 & -1.002881 & -0.904347 \\
\hline-7.385572 & -1.488351 & -2.090059 \\
\hline 0.116734 & -4.283344 & 1.282141 \\
\hline 0.653435 & -6.879090 & 1.839066 \\
\hline 1.978891 & -8.419405 & 0.012879 \\
\hline 2.768371 & -7.345275 & -2.365734 \\
\hline 2.237080 & -4.747091 & -2.924834 \\
\hline-4.485013 & -1.441664 & -4.257866 \\
\hline-7.111847 & -2.098608 & -4.355074 \\
\hline-8.677328 & -1.816156 & -2.143049 \\
\hline-7.593931 & -0.867035 & 0.190183 \\
\hline-4.979027 & -0.210122 & 0.287436 \\
\hline-1.467080 & 5.747253 & -2.428784 \\
\hline-2.143984 & 8.210398 & -1.517547 \\
\hline-2.186715 & 8.708571 & 1.163371 \\
\hline-1.555498 & 6.731302 & 2.935255 \\
\hline-0.882437 & 4.265208 & 2.031910 \\
\hline 4.836299 & 2.168129 & 1.254105 \\
\hline 7.482559 & 2.607012 & 1.273553 \\
\hline 8.971807 & 2.170410 & -0.977131 \\
\hline 7.767586 & 1.248751 & -3.248979 \\
\hline 5.109855 & 0.760373 & -3.268216 \\
\hline-0.243964 & 0.071933 & 0.395052 \\
\hline 3.300857 & -0.809594 & 2.547335 \\
\hline 2.444019 & 0.255325 & 2.344274 \\
\hline 1.069324 & 0.103313 & 2.582325 \\
\hline 0.545470 & -1.139250 & 3.054285 \\
\hline 1.467073 & -2.197332 & 3.265238 \\
\hline 2.818973 & -2.048167 & 3.010260 \\
\hline 2.834236 & 1.190045 & 1.961295 \\
\hline 0.159057 & 1.156821 & 2.258703 \\
\hline-0.874125 & -1.285674 & 3.230049 \\
\hline 1.114359 & -3.162619 & 3.607095 \\
\hline 3.505423 & -2.869612 & 3.166872 \\
\hline-1.727888 & -0.192910 & 2.965251 \\
\hline-1.202808 & 1.030497 & 2.546023 \\
\hline 4.626814 & -0.603974 & 2.182892 \\
\hline 6.969023 & -0.580895 & 2.385076 \\
\hline 7.042176 & 0.511032 & 2.407636 \\
\hline 7.792938 & -1.015975 & 2.951274 \\
\hline 7.022381 & -0.891693 & 1.336874 \\
\hline 5.659208 & -1.013483 & 2.993055 \\
\hline
\end{tabular}




$\begin{array}{lrrr}\mathrm{O} & 5.511780 & -1.608194 & 4.030533 \\ \mathrm{C} & -3.214010 & -0.426557 & 2.992438 \\ \mathrm{C} & -3.630744 & -1.432666 & 4.079552 \\ \mathrm{C} & -4.218793 & 0.734996 & 3.113517 \\ \mathrm{H} & -4.261909 & 1.340255 & 2.203662 \\ \mathrm{C} & -5.549091 & -0.010439 & 3.384288 \\ \mathrm{H} & -6.105862 & -0.186795 & 2.457054 \\ \mathrm{C} & -5.156002 & -1.386739 & 3.955731 \\ \mathrm{O} & -5.921919 & -2.278129 & 4.249907 \\ \mathrm{C} & -3.295976 & -0.955258 & 5.513340 \\ \mathrm{H} & -3.617066 & -1.713386 & 6.235160 \\ \mathrm{H} & -2.221248 & -0.787239 & 5.635318 \\ \mathrm{H} & -3.804165 & -0.018673 & 5.765870 \\ \mathrm{H} & -6.227600 & 0.506850 & 4.070445 \\ \mathrm{H} & -3.954296 & 1.399380 & 3.943955 \\ \mathrm{C} & -2.962012 & -2.767039 & 3.763755 \\ \mathrm{C} & -1.422257 & -2.613893 & 3.735345 \\ \mathrm{H} & -3.313896 & -3.112667 & 2.783722 \\ \mathrm{H} & -3.246874 & -3.536833 & 4.488731 \\ \mathrm{H} & -1.002830 & -3.428713 & 3.137547 \\ \mathrm{H} & -1.022600 & -2.762094 & 4.749418 \\ \mathrm{H} & -1.862312 & 1.862588 & 2.325747 \\ \mathrm{H} & 0.546307 & 2.098599 & 1.887733 \\ \mathrm{H} & -3.443685 & -0.908387 & 2.027359\end{array}$

\section{Structure: ${ }^{3}$ INT4a}

$\begin{array}{lrrr}\mathrm{C} & 2.832896 & 1.438242 & -1.166843 \\ \mathrm{C} & 3.353011 & 2.773612 & -1.357495 \\ \mathrm{C} & 2.278937 & 3.597763 & -1.476296 \\ \mathrm{C} & 1.104887 & 2.762937 & -1.372806 \\ \mathrm{~N} & 1.465080 & 1.455105 & -1.202881 \\ \mathrm{H} & 4.397500 & 3.042610 & -1.408524 \\ \mathrm{H} & 2.278138 & 4.665387 & -1.640317 \\ \mathrm{C} & -2.688077 & 0.695749 & -1.513252 \\ \mathrm{C} & -3.541125 & 1.863930 & -1.523655 \\ \mathrm{C} & -2.721187 & 2.947578 & -1.478672 \\ \mathrm{C} & -1.368396 & 2.441280 & -1.450530 \\ \mathrm{~N} & -1.375791 & 1.070551 & -1.454910 \\ \mathrm{H} & -4.620604 & 1.857185 & -1.546218 \\ \mathrm{H} & -3.005481 & 3.989208 & -1.462945 \\ \mathrm{C} & 1.807382 & -2.751546 & -0.824564 \\ \mathrm{C} & 3.132521 & -3.245051 & -0.511518 \\ \mathrm{C} & 3.956901 & -2.167752 & -0.505104\end{array}$




\begin{tabular}{|c|c|c|c|}
\hline C & 3.136750 & -1.015192 & -0.814495 \\
\hline $\mathrm{N}$ & 1.833378 & -1.393749 & -0.980620 \\
\hline $\mathrm{H}$ & 3.389826 & -4.274710 & -0.311959 \\
\hline $\mathrm{H}$ & 5.016298 & -2.148696 & -0.296332 \\
\hline C & -0.635788 & -3.098297 & -1.206472 \\
\hline $\mathrm{C}$ & -1.790817 & -3.954479 & -1.387642 \\
\hline $\mathrm{C}$ & -2.860706 & -3.134504 & -1.544657 \\
\hline $\mathrm{C}$ & -2.358376 & -1.779025 & -1.463668 \\
\hline $\mathrm{N}$ & -1.001052 & -1.787850 & -1.280620 \\
\hline $\mathrm{H}$ & -1.777242 & -5.034268 & -1.403273 \\
\hline $\mathrm{H}$ & -3.890736 & -3.413136 & -1.713123 \\
\hline $\mathrm{C}$ & 3.622268 & 0.296327 & -0.941299 \\
\hline $\mathrm{C}$ & -3.156724 & -0.632007 & -1.533813 \\
\hline C & -0.216602 & 3.240359 & -1.421146 \\
\hline C & 0.669039 & -3.561720 & -0.957530 \\
\hline $\mathrm{Mn}$ & 0.227841 & -0.162186 & -1.184942 \\
\hline $\mathrm{Cl}$ & 0.445709 & -0.258116 & -3.515618 \\
\hline $\mathrm{C}$ & 0.856114 & -5.033434 & -0.820261 \\
\hline $\mathrm{C}$ & 0.294821 & -5.738783 & 0.249838 \\
\hline $\mathrm{C}$ & 1.599271 & -5.761873 & -1.755588 \\
\hline $\mathrm{C}$ & 0.461865 & -7.114320 & 0.388579 \\
\hline $\mathrm{C}$ & 1.779539 & -7.137781 & -1.635737 \\
\hline $\mathrm{C}$ & 1.207637 & -7.814197 & -0.559104 \\
\hline C & 5.095322 & 0.496268 & -0.843967 \\
\hline $\mathrm{C}$ & 5.669517 & 1.271326 & 0.170330 \\
\hline $\mathrm{C}$ & 5.962345 & -0.067964 & -1.788122 \\
\hline $\mathrm{C}$ & 7.043064 & 1.484816 & 0.239208 \\
\hline $\mathrm{C}$ & 7.340934 & 0.123108 & -1.728890 \\
\hline $\mathrm{C}$ & 7.882843 & 0.903220 & -0.708946 \\
\hline $\mathrm{C}$ & -0.399695 & 4.717752 & -1.406054 \\
\hline $\mathrm{C}$ & -0.923609 & 5.412442 & -2.500303 \\
\hline $\mathrm{C}$ & -0.044673 & 5.460733 & -0.274798 \\
\hline $\mathrm{C}$ & -1.088291 & 6.795590 & -2.475445 \\
\hline $\mathrm{C}$ & -0.200283 & 6.843356 & -0.228167 \\
\hline $\mathrm{C}$ & -0.723650 & 7.510897 & -1.335384 \\
\hline $\mathrm{C}$ & -4.634316 & -0.822890 & -1.588894 \\
\hline C & -5.382409 & -0.462874 & -2.714369 \\
\hline $\mathrm{C}$ & -5.333405 & -1.328145 & -0.488638 \\
\hline $\mathrm{C}$ & -6.770129 & -0.586712 & -2.741783 \\
\hline $\mathrm{C}$ & -6.719454 & -1.446175 & -0.487125 \\
\hline $\mathrm{C}$ & -7.441102 & -1.074581 & -1.620404 \\
\hline $\mathrm{F}$ & -0.423319 & -5.089184 & 1.173429 \\
\hline $\mathrm{F}$ & -0.081473 & -7.764067 & 1.421791 \\
\hline$F$ & 1.374194 & -9.131213 & -0.435353 \\
\hline
\end{tabular}




\begin{tabular}{|c|c|c|c|}
\hline$F$ & 2.490802 & -7.811130 & -2.544005 \\
\hline $\mathrm{F}$ & 2.157140 & -5.136275 & -2.797454 \\
\hline $\mathrm{F}$ & -4.765490 & 0.026798 & -3.794258 \\
\hline $\mathrm{F}$ & -7.460319 & -0.235553 & -3.829466 \\
\hline F & -8.769119 & -1.183253 & -1.632081 \\
\hline $\mathrm{F}$ & -7.357451 & -1.887635 & 0.602761 \\
\hline $\mathrm{F}$ & -4.668711 & -1.685694 & 0.619797 \\
\hline $\mathrm{F}$ & -1.280380 & 4.747267 & -3.604136 \\
\hline $\mathrm{F}$ & -1.587299 & 7.438632 & -3.534341 \\
\hline $\mathrm{F}$ & -0.877383 & 8.834741 & -1.302680 \\
\hline $\mathrm{F}$ & 0.142124 & 7.530299 & 0.864710 \\
\hline $\mathrm{F}$ & 0.453369 & 4.837036 & 0.80105 \\
\hline $\mathrm{F}$ & 4.896858 & 1.840839 & 1.10569 \\
\hline $\mathrm{F}$ & 7.565359 & 2.239706 & 1.21496 \\
\hline $\mathrm{F}$ & 9.199462 & 1.098331 & -0.64292 \\
\hline F & 8.141920 & -0.428017 & -2.64383 \\
\hline F & 5.471334 & -0.809752 & -2.785181 \\
\hline O & 0.035163 & -0.138430 & 0.59275 \\
\hline C & 2.821764 & 0.323815 & 3.74462 \\
\hline $\mathrm{C}$ & 2.030009 & 0.724689 & 2.673497 \\
\hline C & 0.643974 & 0.611340 & 2.742980 \\
\hline $\mathrm{C}$ & 0.019740 & 0.090552 & 3.90742 \\
\hline C & 0.859704 & -0.295466 & 4.97980 \\
\hline C & 2.242118 & -0.190268 & 4.90712 \\
\hline $\mathrm{H}$ & 2.507148 & 1.108259 & 1.777363 \\
\hline $\mathrm{C}$ & -0.166950 & 0.947052 & 1.51186 \\
\hline C & -1.425529 & -0.023096 & 3.97660 \\
\hline $\mathrm{H}$ & 0.426470 & -0.690325 & 5.89171 \\
\hline $\mathrm{H}$ & 2.865076 & -0.490199 & 5.7402 \\
\hline C & -2.209432 & 0.468996 & 2.89633 \\
\hline C & -1.630829 & 1.036370 & 1.78821 \\
\hline O & 4.204200 & 0.383075 & 3.55844 \\
\hline $\mathrm{C}$ & 6.446635 & 0.879346 & 4.06540 \\
\hline $\mathrm{H}$ & 6.584180 & 1.546582 & 3.21053 \\
\hline $\mathrm{H}$ & 7.097992 & 1.190621 & 4.88269 \\
\hline $\mathrm{H}$ & 6.704341 & -0.133200 & 3.73892 \\
\hline $\mathrm{C}$ & 5.010375 & 0.931939 & 4.52232 \\
\hline O & 4.613197 & 1.392076 & 5.56394 \\
\hline C & -3.702274 & 0.276160 & 2.9758 \\
\hline C & -4.244240 & 0.396881 & 4.4115 \\
\hline C & -4.671641 & 1.108997 & 2.11953 \\
\hline $\mathrm{H}$ & -4.593202 & 0.876764 & 1.05427 \\
\hline $\mathrm{C}$ & -6.042678 & 0.723652 & 2.72123 \\
\hline H & -6.476812 & -0.142168 & 2.20499 \\
\hline
\end{tabular}


$-5.751927$

$-6.580157$

$-4.005093$

$-4.403841$

$-2.937590$

$-4.501656$

$-6.796503$

$-4.471020$

$-3.603023$

$-2.061106$

$-3.884792$

$-3.981589$

$-1.617037$

$-1.768759$

$-2.256527$

0.207465

$-3.881088$
0.284612

$-0.084407$

1. 790189

1.801838

2.027946

2.590415

1. 517285

2.180495

$-0.710482$

$-0.565228$

$-1.679317$

$-0.703217$

$-1.538743$

0.098694

1.427607

1.877694

$-0.769977$
4.172927

4.976622

5.043245

6.063003

5.077766

4.483803

2.702216

2. 229482

5.245535

5.247336

4.814396

6.273459

5.496142

6.075553

0.996101

1.064110

2.680176

\section{Structure: ${ }^{5}$ INT4a}

C

C

C

C

$\mathrm{N}$

$\mathrm{H}$

$\mathrm{H}$

C

C

C

C

$\mathrm{N}$

$\mathrm{H}$

$\mathrm{H}$

C

C

C

C

$\mathrm{N}$

$\mathrm{H}$

$\mathrm{H}$

C

C

C

$$
\begin{array}{rrr}
-2.784448 & -1.521550 & -1.140528 \\
-3.266493 & -2.869087 & -1.315806 \\
-2.176978 & -3.674076 & -1.420289 \\
-1.015198 & -2.827358 & -1.327101 \\
-1.410060 & -1.520285 & -1.168906 \\
-4.306000 & -3.157495 & -1.361829 \\
-2.153789 & -4.744148 & -1.565673 \\
2.723619 & -0.684497 & -1.474182 \\
3.587615 & -1.840052 & -1.442557 \\
2.786449 & -2.936820 & -1.385427 \\
1.424041 & -2.464529 & -1.390322 \\
1.413300 & -1.092307 & -1.432852 \\
4.667041 & -1.811287 & -1.452596 \\
3.085915 & -3.973596 & -1.343179 \\
-1.820788 & 2.671855 & -0.857460 \\
-3.147177 & 3.132071 & -0.517400 \\
-3.951550 & 2.039707 & -0.486307 \\
-3.127832 & 0.898715 & -0.809263 \\
-1.834528 & 1.309287 & -1.011200 \\
-3.416291 & 4.159079 & -0.319199 \\
-5.005584 & 1.999438 & -0.254525 \\
0.580749 & 3.055889 & -1.270487 \\
1.721675 & 3.918620 & -1.471866 \\
2.808814 & 3.116025 & -1.603194
\end{array}
$$


C

N

$\mathrm{H}$

$\mathrm{H}$

C

C

C

C

Mn

$\mathrm{Cl}$

C

C

C

C

C

C

C

C

C

C

C

C

C

C

C

C

C

C

C

C

C

C

C

C

F

F

F

F

F

F

F

F

F

E
2.343530

0.983585

1.685390

3.835100

$-3.603254$

3.176483

0.300546

$-0.716781$

$-0.210767$

$-0.439208$

$-0.935756$

$-0.396128$

$-1.694369$

$-0.594091$

$-1.911474$

$-1.356780$

$-5.071656$

$-5.633609$

$-5.952397$

$-7.002573$

$-7.328104$

$-7.855249$

0.512312

1.035802

0.189998

1.231833

0.371287

0.895193

4.649839

5.411163

5.337152

6.797315

6.720599

7.454676

0.337885

$-0.065854$

$-1.556050$

$-2.640823$

$-2.238198$

4.810423

7.499400

8.781427

7.345060

4.661711 $\begin{array}{ll}1.754227 & -1.491145\end{array}$

$1.747115-1.311018$

$\begin{array}{ll}4.997315 & -1.510747\end{array}$

$3.408568-1.771161$

$-0.409813-0.921780$

$0.635924-1.528246$

$-3.292810-1.363736$

$3.510496-1.022209$

$0.110748-1.213466$

$0.168501-3.526232$

$\begin{array}{ll}4.980270 & -0.917247\end{array}$

$\begin{array}{ll}5.724994 & 0.136833\end{array}$

$5.670586-1.868995$

$\begin{array}{rr}7.099601 & 0.242576\end{array}$

$\begin{array}{ll}7.043751 & -1.779909\end{array}$

$\begin{array}{ll}7.759248 & -0.719983\end{array}$

$-0.634659-0.818753$

$\begin{array}{rr}-1.411691 & 0.200487\end{array}$

$-0.091929-1.763136$

$\begin{array}{ll}-1.652596 & 0.271476\end{array}$

$-0.303140-1.698479$

$-1.088539-0.675154$

$-4.765645-1.325516$

$\begin{array}{ll}-5.470187 & -2.413539\end{array}$

$\begin{array}{ll}-5.497505 & -0.177623\end{array}$

$-6.849019-2.366769$

$\begin{array}{ll}-6.876240 & -0.109547\end{array}$

$-7.552332-1.211020$

$0.855349-1.577705$

$\begin{array}{lll}0.507657 & -2.698262\end{array}$

$\begin{array}{lll}1.374711 & -0.476924\end{array}$

$0.653677-2.718810$

$1.519347-0.468470$

$1.157128-1.596587$

$\begin{array}{ll}5.116240 & 1.077314\end{array}$

$\begin{array}{ll}7.786652 & 1.260614\end{array}$

$9.075173-0.626237$

$\begin{array}{ll}7.678120 & -2.702852\end{array}$

$5.008326-2.896204$

$0.002424-3.780507$

$0.309818-3.801938$

$1.289027-1.602004$

$\begin{array}{ll}1.975851 & 0.624324\end{array}$

$\begin{array}{ll}1.725752 & 0.628940\end{array}$ 


$$
\begin{aligned}
& 1.367980 \\
& 1.733199 \\
& 1.076457 \\
& 0.055228
\end{aligned}
$$

$-0.307728$

$-4.851213$

$-7.508364$

$-9.169148$

$-8.140773$

$-5.478216$

$-0.017613$

$-2.875379$

$-2.062078$

$-0.676209$

$-0.076903$

$-0.942940$

$-2.320425$

$-2.519409$

0.162527

1. 360707

$-0.530557$

$-2.963293$

2. 172642

1. 620185

$-4.250556$

$-6.505631$

$-6.637690$

$-7.176006$

$-6.742595$

$-5.079928$

$-4.701598$

3. 660436

4.175172

4. 655998

4.593518

6.009866

6.437948

5.687510

6.495164

3. 932156

4.326513

2.864450

4.430273

6.776243
$-4.817656$

$-7.500095$

$-8.872882$

$-7.551456$

$-4.866364$

$-1.964761$

$-2.412587$

$-1.305350$

0.232388

0.654922

0.111017

$-0.168383$

$-0.650226$

$-0.533566$

0.072274

0.542549

0.434070

$-1.099464$

$-0.951545$

0.186365

1. 009281

0.799684

$-0.366023$

$-1.007238$

$-0.240609$

$-0.692140$

$-1.467014$

$-0.886520$

0.270707

$-0.705695$

$-1.078255$

$-0.152263$

$-0.200478$

$-1.006561$

$-0.818606$

$-0.575936$

0.276315

$-0.086758$

0.316626

$-1.562332$

$-1.527141$

$-1.798938$

$-2.387463$

$-1.357136$
$-3.533011$

$-3.420440$

$-1.157556$

0.999585

0.895921

1.139799

1.253759

$-0.604631$

$-2.613356$

$-2.765306$

0.570258

3.639750

2. 621659

2. 717390

3. 855354

4.875316

4.775570

1.746596

1.537488

3.950993

5.762397

5.565946

2. 916078

1.839213

3.429684

3.933189

3. 173262

4.771062

3.470253

4. 421314

5.503619

3.014615

4.464293

2. 211936

1.137121

2. 821575

2. 278277

4.249347

5.057088

5.158331

6.179191

5.200651

4.638052

2. 845067 
$\mathrm{H}$

C

C

$\mathrm{H}$

$\mathrm{H}$

$\mathrm{H}$

$\mathrm{H}$

$\mathrm{H}$

$\mathrm{H}$

$\mathrm{H}$

$\begin{array}{rrr}4.469235 & -2.075526 & 2.364030 \\ 3.513357 & 0.943330 & 5.230436 \\ 1.971214 & 0.800381 & 5.201098 \\ 3.806985 & 1.891978 & 4.764139 \\ 3.864530 & 0.982530 & 6.267132 \\ 1.523743 & 1.786844 & 5.382177 \\ 1.654458 & 0.184645 & 6.056661 \\ 2.260392 & -1.449432 & 1.085660 \\ -0.205906 & -1.890402 & 1.106915 \\ 3.828504 & 0.882203 & 2.676109\end{array}$

\section{Structure: ${ }^{3}$ INT4}

C

C

C

C

$\mathrm{N}$

$\mathrm{H}$

$\mathrm{H}$

C

C

C

C

$\mathrm{N}$

$\mathrm{H}$

$\mathrm{H}$

C

C

C

C

$\mathrm{N}$

$\mathrm{H}$

$\mathrm{H}$

C

C

C

C

N

$\mathrm{H}$

$\mathrm{H}$

C

C

C

$$
\begin{array}{rrr}
-0.090987 & -2.181567 & -1.808943 \\
0.856089 & -3.147024 & -2.303969 \\
2.055205 & -2.510423 & -2.409313 \\
1.859857 & -1.158188 & -1.946610 \\
0.542849 & -0.978010 & -1.582842 \\
0.619917 & -4.166257 & -2.575110 \\
2.984269 & -2.916843 & -2.782634 \\
1.745154 & 2.975514 & -0.671783 \\
3.165538 & 3.237357 & -0.774921 \\
3.746189 & 2.090736 & -1.215451 \\
2.680766 & 1.125543 & -1.397602 \\
1.482741 & 1.687870 & -1.053739 \\
3.649141 & 4.177183 & -0.551287 \\
4.795472 & 1.916823 & -1.404347 \\
-3.370349 & 0.446947 & -0.822228 \\
-4.431617 & -0.526511 & -0.984742 \\
-3.839650 & -1.710206 & -1.286139 \\
-2.415534 & -1.458554 & -1.348623 \\
-2.160743 & -0.140194 & -1.081185 \\
-5.488891 & -0.326827 & -0.888735 \\
-4.317259 & -2.662789 & -1.451763 \\
-2.548271 & 2.708592 & -0.201752 \\
-2.754691 & 4.044616 & 0.319156 \\
-1.537628 & 4.644068 & 0.355202 \\
-0.579323 & 3.676760 & -0.139777 \\
-1.221080 & 2.514366 & -0.467261 \\
-3.703451 & 4.461464 & 0.623814 \\
-1.303687 & 5.642341 & 0.695442 \\
-1.451363 & -2.438589 & -1.618689 \\
0.798783 & 3.909470 & -0.232139 \\
2.870563 & -0.196958 & -1.830342
\end{array}
$$




\begin{tabular}{|c|c|c|c|}
\hline C & -3.565386 & 1.759201 & -0.372759 \\
\hline $\mathrm{Mn}$ & -0.343946 & 0.790068 & -1.059271 \\
\hline $\mathrm{Cl}$ & -0.607777 & 1.332305 & -3.266719 \\
\hline $\mathrm{C}$ & -4.953132 & 2.131107 & 0.022177 \\
\hline $\mathrm{C}$ & -5.599912 & 1.470431 & 1.072978 \\
\hline C & -5.682260 & 3.122099 & -0.643825 \\
\hline $\mathrm{C}$ & -6.913693 & 1.751554 & 1.431736 \\
\hline C & -6.991499 & 3.440737 & -0.285962 \\
\hline $\mathrm{C}$ & -7.611585 & 2.748513 & 0.752738 \\
\hline $\mathrm{C}$ & -1.876260 & -3.865964 & -1.604712 \\
\hline $\mathrm{C}$ & -1.448150 & -4.695617 & -0.563360 \\
\hline $\mathrm{C}$ & -2.735031 & -4.427974 & -2.551687 \\
\hline $\mathrm{C}$ & -1.862804 & -6.016507 & -0.449691 \\
\hline $\mathrm{C}$ & -3.168262 & -5.748748 & -2.458991 \\
\hline $\mathrm{C}$ & -2.729552 & -6.544964 & -1.402870 \\
\hline $\mathrm{C}$ & 4.265625 & -0.640014 & -2.102374 \\
\hline $\mathrm{C}$ & 5.049420 & -0.114305 & -3.134531 \\
\hline $\mathrm{C}$ & 4.864491 & -1.603278 & -1.287364 \\
\hline $\mathrm{C}$ & 6.373309 & -0.510951 & -3.325656 \\
\hline $\mathrm{C}$ & 6.183696 & -2.008286 & -1.446053 \\
\hline $\mathrm{C}$ & 6.943855 & -1.459504 & -2.477510 \\
\hline $\mathrm{C}$ & 1.300851 & 5.251271 & 0.183220 \\
\hline $\mathrm{C}$ & 1.017274 & 6.401784 & -0.559732 \\
\hline $\mathrm{C}$ & 2.075428 & 5.410253 & 1.336343 \\
\hline C & 1.478681 & 7.658810 & -0.173545 \\
\hline $\mathrm{C}$ & 2.554677 & 6.654327 & 1.738226 \\
\hline $\mathrm{C}$ & 2.252046 & 7.783232 & 0.979064 \\
\hline $\mathrm{F}$ & -4.963985 & 0.500010 & 1.756268 \\
\hline $\mathrm{F}$ & -7.505093 & 1.067789 & 2.420576 \\
\hline $\mathrm{F}$ & -8.870307 & 3.035507 & 1.092991 \\
\hline $\mathrm{F}$ & -7.661002 & 4.394511 & -0.940991 \\
\hline $\mathrm{F}$ & -5.125555 & 3.793386 & -1.657563 \\
\hline $\mathrm{F}$ & 0.278181 & 6.313758 & -1.670822 \\
\hline $\mathrm{F}$ & 1.191305 & 8.742596 & -0.902128 \\
\hline $\mathrm{F}$ & 2.703250 & 8.982384 & 1.355917 \\
\hline $\mathrm{F}$ & 3.295279 & 6.773832 & 2.847015 \\
\hline $\mathrm{F}$ & 2.382220 & 4.342123 & 2.088861 \\
\hline $\mathrm{F}$ & 4.542209 & 0.806963 & -3.957985 \\
\hline $\mathrm{F}$ & 7.100197 & 0.012137 & -4.317075 \\
\hline $\mathrm{F}$ & 8.212367 & -1.836632 & -2.648966 \\
\hline $\mathrm{F}$ & 6.726054 & -2.903741 & -0.606530 \\
\hline$F$ & 4.154322 & -2.149734 & -0.279926 \\
\hline $\mathrm{F}$ & -0.619438 & -4.215008 & 0.381107 \\
\hline$F$ & -1.444462 & -6.775306 & 0.571726 \\
\hline
\end{tabular}




\begin{tabular}{|c|c|c|c|}
\hline$F$ & -3.137816 & -7.813301 & -1.306266 \\
\hline $\mathrm{F}$ & -3.995024 & -6.258963 & -3.379091 \\
\hline $\mathrm{F}$ & -3.183798 & -3.684090 & -3.571900 \\
\hline O & -0.130454 & 0.435388 & 0.786851 \\
\hline C & -2.531598 & -2.505509 & 2.537352 \\
\hline C & -1.751459 & -1.555384 & 1.909820 \\
\hline $\mathrm{C}$ & -0.365623 & -1.602589 & 2.065844 \\
\hline C & 0.243258 & -2.576098 & 2.914359 \\
\hline $\mathrm{C}$ & -0.599037 & -3.575935 & 3.485415 \\
\hline $\mathrm{C}$ & -1.962357 & -3.549918 & 3.291784 \\
\hline $\mathrm{H}$ & -2.197235 & -0.764044 & 1.322721 \\
\hline $\mathrm{C}$ & 0.485319 & -0.661480 & 1.289615 \\
\hline $\mathrm{C}$ & 1.633727 & -2.458870 & 3.239335 \\
\hline $\mathrm{H}$ & -0.173788 & -4.371323 & 4.085391 \\
\hline $\mathrm{H}$ & -2.608515 & -4.301630 & 3.731289 \\
\hline $\mathrm{C}$ & 2.421923 & -1.409704 & 2.689887 \\
\hline $\mathrm{C}$ & 1.860025 & -0.531104 & 1.783008 \\
\hline O & -3.907181 & -2.367184 & 2.470391 \\
\hline C & -6.057528 & -2.732040 & 1.587926 \\
\hline $\mathrm{H}$ & -6.571843 & -3.192186 & 0.743621 \\
\hline $\mathrm{H}$ & -6.523994 & -3.064109 & 2.522459 \\
\hline $\mathrm{H}$ & -6.132236 & -1.642611 & 1.550784 \\
\hline C & -4.610538 & -3.143923 & 1.578176 \\
\hline 0 & -4.101257 & -4.015178 & 0.917507 \\
\hline $\mathrm{C}$ & 3.833065 & -1.229869 & 3.199769 \\
\hline $\mathrm{C}$ & 4.512351 & -2.571681 & 3.538722 \\
\hline C & 4.882333 & -0.442205 & 2.391895 \\
\hline $\mathrm{H}$ & 4.685357 & 0.634078 & 2.383918 \\
\hline $\mathrm{C}$ & 6.202167 & -0.799310 & 3.119589 \\
\hline $\mathrm{H}$ & 6.501325 & -0.029286 & 3.840253 \\
\hline $\mathrm{C}$ & 5.919876 & -2.095600 & 3.906042 \\
\hline O & 6.676000 & -2.637433 & 4.680290 \\
\hline $\mathrm{C}$ & 4.684546 & -3.519681 & 2.326676 \\
\hline $\mathrm{H}$ & 5.098526 & -4.471254 & 2.676098 \\
\hline $\mathrm{H}$ & 3.731268 & -3.714191 & 1.825308 \\
\hline $\mathrm{H}$ & 5.363843 & -3.120215 & 1.571087 \\
\hline $\mathrm{H}$ & 7.053040 & -0.946900 & 2.446170 \\
\hline $\mathrm{H}$ & 4.894281 & -0.781212 & 1.355592 \\
\hline C & 3.703319 & -3.233739 & 4.647807 \\
\hline C & 2.254174 & -3.487631 & 4.173251 \\
\hline $\mathrm{H}$ & 3.696272 & -2.579494 & 5.527989 \\
\hline $\mathrm{H}$ & 4.157156 & -4.178608 & 4.963215 \\
\hline $\mathrm{H}$ & 1.607366 & -3.608997 & 5.049080 \\
\hline $\mathrm{H}$ & 2.205409 & -4.450166 & 3.642998 \\
\hline
\end{tabular}




$\begin{array}{lrrr}\mathrm{H} & 2.450009 & 0.255859 & 1.322555 \\ \mathrm{H} & 0.781032 & -1.346263 & 0.404298 \\ \mathrm{H} & 3.733697 & -0.699505 & 4.162924\end{array}$

\section{Structure: ${ }^{5}$ INT4}

C

C

C

$\mathrm{N}$

$\mathrm{H}$

$\mathrm{H}$

C

C

C

C

$\mathrm{N}$

$\mathrm{H}$

$\mathrm{H}$

C

C

C

C

$\mathrm{N}$

$\mathrm{H}$

$\mathrm{H}$

C

C

C

C

$\mathrm{N}$

$\mathrm{H}$

$\mathrm{H}$

C

C

C

C

$\mathrm{Mn}$

$\mathrm{Cl}$

C

C

C

C

\begin{tabular}{|c|c|c|}
\hline-0.437977 & -2.475254 & -1.619155 \\
\hline 0.350730 & -3.618708 & -2.037691 \\
\hline 1.647977 & -3.201496 & -2.116858 \\
\hline 1.668841 & -1.803300 & -1.731434 \\
\hline 0.401892 & -1.419574 & -1.39 \\
\hline-0.032927 & -4.599646 & $-2 \cdot 2$ \\
\hline 2.501489 & -3.780449 & -2.44 \\
\hline 2.149192 & 2.54156 & $-1 \cdot 0$ \\
\hline 570 & 2.538 & -1 \\
\hline 3.98 & 1.2631 & -1.3792 \\
\hline 2.79 & 0.4543 & -1.4 \\
\hline 1.70 & 1.26 & $-1 \cdot 2$ \\
\hline 4.23 & 3.4 & -0 \\
\hline 5.0 & 0.9 & -1.4 \\
\hline-3.24 & 0.8609 & -0.8 \\
\hline-4.44 & 0.0 & $-1 \cdot 0$ \\
\hline-4.0 & $-1 \cdot 21$ & $-1 \cdot 3$ \\
\hline-2.6 & -1.22 & $-1 \cdot 3$ \\
\hline-2.15 & 0.04 & $-1 \cdot 0$ \\
\hline-5.4 & 0.4 & -0 \\
\hline-4.6 & -2.0 & -1 \\
\hline$-2 \cdot 12$ & 3.0 & -0.4 \\
\hline-2.0 & 4.4 & 0 \\
\hline-0.7 & & -0 \\
\hline-0.02 & 3.7 & -0.5 \\
\hline-0.87 & 2.72 & -0.8 \\
\hline-2.941912 & 5.02 & 0.4 \\
\hline-0.395333 & 5.81 & 0.2 \\
\hline-1.839668 & -2.39 & -1.525038 \\
\hline 1.375348 & 3.672972 & -0.6 \\
\hline 2.786243 & -0.945416 & $-1 \cdot 7$ \\
\hline-3.245371 & 2.229060 & -0.505604 \\
\hline-0.234323 & 0.705733 & -1.20984 \\
\hline-0.417125 & 0.870324 & -3.51558 \\
\hline-4.554632 & 2.773621 & -0.04768 \\
\hline-5.210520 & 2.219526 & 1.05937 \\
\hline-5.198250 & 3.833624 & -0.69562 \\
\hline-6.457244 & 2.665036 & 1.48382 \\
\hline
\end{tabular}




\begin{tabular}{|c|c|c|c|}
\hline $\mathrm{C}$ & -6.437364 & 4.313643 & -0.273464 \\
\hline C & -7.073395 & 3.722148 & 0.817022 \\
\hline C & -2.604218 & -3.669737 & -1.677003 \\
\hline C & -3.296401 & -4.229235 & -0.597732 \\
\hline C & -2.677830 & -4.349283 & -2.897056 \\
\hline C & -4.032007 & -5.404956 & -0.714584 \\
\hline C & -3.397005 & -5.535115 & -3.038165 \\
\hline C & -4.078392 & -6.062435 & -1.942950 \\
\hline $\mathrm{C}$ & 4.111324 & -1.601693 & -1.888716 \\
\hline C & 4.969885 & -1.330739 & -2.959407 \\
\hline C & 4.557040 & -2.528913 & -0.943253 \\
\hline C & 6.222523 & -1.935229 & -3.066412 \\
\hline $\mathrm{C}$ & 5.804792 & -3.135325 & -1.017278 \\
\hline C & 6.642557 & -2.839381 & -2.091269 \\
\hline C & 2.128047 & 4.914038 & -0.328308 \\
\hline C & 2.029340 & 6.070451 & -1.108665 \\
\hline $\mathrm{C}$ & 2.939154 & 4.979504 & 0.809765 \\
\hline C & 2.708873 & 7.241616 & -0.778324 \\
\hline C & 3.634071 & 6.135044 & 1.155818 \\
\hline C & 3.515396 & 7.271571 & 0.357690 \\
\hline $\mathrm{F}$ & -4.650588 & 1.207520 & 1.741613 \\
\hline $\mathrm{F}$ & -7.062830 & 2.078939 & 2.528042 \\
\hline $\mathrm{F}$ & -8.266938 & 4.164750 & 1.218625 \\
\hline $\mathrm{F}$ & -7.027594 & 5.325738 & -0.917280 \\
\hline $\mathrm{F}$ & -4.629313 & 4.411205 & -1.759513 \\
\hline $\mathrm{F}$ & 1.262588 & 6.072526 & -2.204572 \\
\hline $\mathrm{F}$ & 2.596844 & 8.331877 & -1.544082 \\
\hline $\mathrm{F}$ & 4.173611 & 8.387084 & 0.682269 \\
\hline $\mathrm{F}$ & 4.404974 & 6.164796 & 2.249848 \\
\hline $\mathrm{F}$ & 3.068459 & 3.904635 & 1.603657 \\
\hline $\mathrm{F}$ & 4.606977 & -0.459383 & -3.904629 \\
\hline $\mathrm{F}$ & 7.024128 & -1.655006 & -4.097814 \\
\hline $\mathrm{F}$ & 7.843518 & -3.413726 & -2.181532 \\
\hline $\mathrm{F}$ & 6.209009 & -3.979781 & -0.056265 \\
\hline $\mathrm{F}$ & 3.773262 & -2.824511 & 0.112693 \\
\hline $\mathrm{F}$ & -3.277440 & -3.619134 & 0.600749 \\
\hline $\mathrm{F}$ & -4.686193 & -5.905583 & 0.338816 \\
\hline $\mathrm{F}$ & -4.774536 & -7.194560 & -2.069485 \\
\hline $\mathrm{F}$ & -3.444284 & -6.163960 & -4.216930 \\
\hline F & -2.041527 & -3.866546 & -3.970300 \\
\hline O & -0.023788 & 0.621019 & 0.718354 \\
\hline C & -2.751239 & -1.766584 & 2.777806 \\
\hline C & -1.841725 & -1.023519 & 2.041941 \\
\hline C & -0.480507 & -1.176422 & 2.260350 \\
\hline
\end{tabular}




\begin{tabular}{|c|c|c|c|}
\hline C & 0.005716 & -2.042496 & 3.286959 \\
\hline C & -0.961491 & -2.808483 & 4.003353 \\
\hline C & -2.314008 & -2.692128 & 3.756537 \\
\hline $\mathrm{H}$ & -2.185897 & -0.320429 & 1.297368 \\
\hline C & 0.469136 & -0.466994 & 1.364009 \\
\hline C & 1.398875 & -2.038937 & 3.608791 \\
\hline $\mathrm{H}$ & -0.642245 & -3.509294 & 4.766020 \\
\hline $\mathrm{H}$ & -3.033347 & -3.288389 & 4.298220 \\
\hline C & 2.309782 & -1.219130 & 2.881449 \\
\hline C & 1.852211 & -0.433438 & 1.841529 \\
\hline O & -4.053666 & -1.472971 & 2.475095 \\
\hline C & -6.349354 & -1.618116 & 2.093904 \\
\hline $\mathrm{H}$ & -7.246279 & -2.189180 & 2.334049 \\
\hline $\mathrm{H}$ & -6.442513 & -0.582310 & 2.434865 \\
\hline $\mathrm{H}$ & -6.198312 & -1.583489 & 1.009024 \\
\hline C & -5.147748 & -2.270311 & 2.725338 \\
\hline O & -5.114682 & -3.308160 & 3.335256 \\
\hline C & 3.745101 & -1.146918 & 3.348787 \\
\hline C & 4.238360 & -2.477636 & 3.951657 \\
\hline $\mathrm{C}$ & 4.871301 & -0.702343 & 2.395731 \\
\hline $\mathrm{H}$ & 4.827596 & 0.364955 & 2.158797 \\
\hline C & 6.146988 & -1.084831 & 3.186552 \\
\hline $\mathrm{H}$ & 6.566030 & -0.233315 & 3.735297 \\
\hline C & 5.706528 & -2.141169 & 4.221563 \\
\hline O & 6.399023 & -2.614778 & 5.093847 \\
\hline C & 4.249151 & -3.661369 & 2.953257 \\
\hline $\mathrm{H}$ & 4.535276 & -4.573518 & 3.487274 \\
\hline $\mathrm{H}$ & 3.266761 & -3.814884 & 2.496434 \\
\hline $\mathrm{H}$ & 4.959308 & -3.519936 & 2.136246 \\
\hline $\mathrm{H}$ & 6.953349 & -1.483997 & 2.562270 \\
\hline $\mathrm{H}$ & 4.805693 & -1.250068 & 1.454413 \\
\hline C & 3.369260 & -2.788047 & 5.164194 \\
\hline $\mathrm{C}$ & 1.891548 & -2.936972 & 4.734774 \\
\hline $\mathrm{H}$ & 3.464093 & -1.974262 & 5.893357 \\
\hline $\mathrm{H}$ & 3.699708 & -3.701475 & 5.668916 \\
\hline $\mathrm{H}$ & 1.247543 & -2.793032 & 5.609571 \\
\hline $\mathrm{H}$ & 1.707575 & -3.971010 & 4.408891 \\
\hline $\mathrm{H}$ & 2.530769 & 0.198851 & 1.276983 \\
\hline $\mathrm{H}$ & 0.648451 & -1.291903 & 0.561631 \\
\hline $\mathrm{H}$ & 3.752187 & -0.425396 & 4.184347 \\
\hline
\end{tabular}

\section{Structure: ${ }^{3} \mathrm{TS} 5$}

C

$$
0.003874-2.244846 \quad-1.565108
$$




\begin{tabular}{|c|c|c|c|}
\hline $\mathrm{C}$ & 0.957030 & -3.134237 & -2.156450 \\
\hline C & 2.125965 & -2.442078 & -2.298568 \\
\hline $\mathrm{C}$ & 1.921977 & -1.132622 & -1.752030 \\
\hline $\mathrm{N}$ & 0.617492 & -1.030955 & -1.266421 \\
\hline $\mathrm{H}$ & 0.743548 & -4.142213 & -2.482768 \\
\hline $\mathrm{H}$ & 3.037263 & -2.790620 & -2.762362 \\
\hline $\mathrm{C}$ & 1.632750 & 3.002056 & -0.503694 \\
\hline $\mathrm{C}$ & 3.048560 & 3.300966 & -0.550730 \\
\hline $\mathrm{C}$ & 3.672873 & 2.176952 & -0.990728 \\
\hline $\mathrm{C}$ & 2.640910 & 1.185483 & -1.216948 \\
\hline $\mathrm{N}$ & 1.419557 & 1.708585 & -0.899104 \\
\hline $\mathrm{H}$ & 3.499918 & 4.249168 & -0.297261 \\
\hline $\mathrm{H}$ & 4.732107 & 2.029854 & -1.143692 \\
\hline $\mathrm{C}$ & -3.393041 & 0.307438 & -0.767182 \\
\hline $\mathrm{C}$ & -4.411769 & -0.714043 & -0.908027 \\
\hline $\mathrm{C}$ & -3.766923 & -1.886837 & -1.128298 \\
\hline $\mathrm{C}$ & -2.351025 & -1.583665 & -1.164932 \\
\hline $\mathrm{N}$ & -2.153912 & -0.245937 & -0.953970 \\
\hline $\mathrm{H}$ & -5.478138 & -0.552531 & -0.851575 \\
\hline $\mathrm{H}$ & -4.202386 & -2.865613 & -1.248653 \\
\hline $\mathrm{C}$ & -2.673036 & 2.627979 & -0.247888 \\
\hline $\mathrm{C}$ & -2.941359 & 3.974802 & 0.210940 \\
\hline C & -1.743206 & 4.607540 & 0.295431 \\
\hline $\mathrm{C}$ & -0.734770 & 3.652565 & -0.113434 \\
\hline $\mathrm{N}$ & -1.329487 & 2.464612 & -0.441313 \\
\hline $\mathrm{H}$ & -3.915362 & 4.374598 & 0.451718 \\
\hline $\mathrm{H}$ & -1.554261 & 5.621344 & 0.616694 \\
\hline $\mathrm{C}$ & -1.350226 & -2.538668 & -1.398070 \\
\hline $\mathrm{C}$ & 0.641589 & 3.915802 & -0.126759 \\
\hline $\mathrm{C}$ & 2.883812 & -0.123041 & -1.677340 \\
\hline C & -3.651901 & 1.636042 & -0.411600 \\
\hline $\mathrm{Mn}$ & -0.380536 & 0.760503 & -0.925043 \\
\hline $\mathrm{Cl}$ & -0.565916 & 1.137230 & -3.166301 \\
\hline $\mathrm{C}$ & -5.068322 & 1.992542 & -0.115116 \\
\hline $\mathrm{C}$ & -5.760139 & 1.387284 & 0.940806 \\
\hline $\mathrm{C}$ & -5.773578 & 2.927949 & -0.880051 \\
\hline $\mathrm{C}$ & -7.092590 & 1.676360 & 1.217212 \\
\hline $\mathrm{C}$ & -7.102714 & 3.249460 & -0.610303 \\
\hline $\mathrm{C}$ & -7.765379 & 2.618027 & 0.440666 \\
\hline $\mathrm{C}$ & -1.736397 & -3.975546 & -1.448725 \\
\hline C & -1.281159 & -4.842856 & -0.450494 \\
\hline C & -2.577514 & -4.515208 & -2.425181 \\
\hline C & -1.662167 & -6.178057 & -0.400087 \\
\hline $\mathrm{C}$ & -2.975497 & -5.849524 & -2.39582 \\
\hline
\end{tabular}


C

C

C

C

C

C

C

C

C

C

C

C

C

F

F

F

F

F

F

F

F

$\mathrm{F}$

F

F

F

F

F

F

F

F

F

F

F

$\mathrm{O}$

C

C

C

C

C

C

$\mathrm{H}$

C

C

$\mathrm{H}$
$-2.516306$

4.271387

4.933571

4.989798

6.251096

6.306778

6.942242

1.089691

0.830947

1.790911

1.246148

2. 223092

1. 946651

$-5.147842$

$-7.725158$

$-9.041360$

$-7.748421$

$-5.171655$

0.161106

0.984125

2.353261

2.894439

2.071579

4.309975

6.856282

8.205306

6.959685

4.406996

$-0.459082$

$-1.221348$

$-2.890862$

$-3.785938$

$-3.038914$

$-0.205340$

$-2.385475$

$-1.676381$

$-0.279223$

0.404971

$-0.370746$

$-1.740624$

$-2.184086$

0.481218

1.806528

0.114330
$-6.682619-1.377282$

$-0.459880 \quad-2.096604$

$0.222118-3.125672$

$-1.474815-1.459158$

$-0.072833-3.474706$

$-1.782654-1.777560$

$\begin{array}{ll}-1.077188 & -2.797529\end{array}$

$\begin{array}{ll}5.273650 & 0.298765\end{array}$

$6.408216-0.476585$

$5.460827 \quad 1.493755$

$7.678445-0.081552$

$6.718871 \quad 1.905641$

$7.831865 \quad 1.113527$

$0.472267 \quad 1.714768$

1.059457

2.222888

$2.911086 \quad 0.700440$

$4.149479-1.358123$

$3.543019-1.903847$

$6.290822-1.628548$

$8.746740-0.841424$

$9.043581 \quad 1.499418$

$6.866385 \quad 3.053742$

$4.407808 \quad 2.275798$

$1.194854-3.795743$

$0.599204-4.457980$

$-1.357848-3.121904$

$-2.736857-1.100620$

$-2.179481-0.471276$

$\begin{array}{ll}-4.387361 & 0.508441\end{array}$

$\begin{array}{ll}-6.974199 & 0.581724\end{array}$

$-7.963949-1.341802$

$-6.337935-3.341777$

$-3.738541-3.414970$

$0.494632 \quad 0.942132$

$-2.639898 \quad 2.628228$

$-1.626657$

2.024525

2.132724

2.911735

3.467348

3.321891

1.494805

1.434072

3.185176

4.016029 
O

C

$\mathrm{H}$

$\mathrm{H}$

$\mathrm{H}$

C

O

C

C

C

$\mathrm{H}$

C

$\mathrm{H}$

C

O

C

$\mathrm{H}$

$\mathrm{H}$

$\mathrm{H}$

$\mathrm{H}$

$\mathrm{H}$

C

C

$\mathrm{H}$

$\mathrm{H}$

$\mathrm{H}$

$\mathrm{H}$

$\mathrm{H}$

$\mathrm{H}$

$\mathrm{H}$

$\begin{array}{rrr}-2.332086 & -4.482753 & 3.749938 \\ 2.516576 & -1.348036 & 2.631175 \\ 1.862557 & -0.425089 & 1.812643 \\ -3.773419 & -2.565967 & 2.600242 \\ -5.914752 & -2.972668 & 1.726885 \\ -6.443042 & -3.512004 & 0.940146 \\ -6.350615 & -3.219715 & 2.701104 \\ -6.006692 & -1.891137 & 1.591126 \\ -4.458320 & -3.353073 & 1.710810 \\ -3.934076 & -4.204373 & 1.033318 \\ 3.947666 & -1.116847 & 3.061342 \\ 4.689597 & -2.426631 & 3.394784 \\ 4.934084 & -0.315055 & 2.190522 \\ 4.697003 & 0.752635 & 2.155137 \\ 6.290413 & -0.599417 & 2.883380 \\ 6.576065 & 0.199532 & 3.577667 \\ 6.086287 & -1.888060 & 3.709091 \\ 6.889907 & -2.378574 & 4.469864 \\ 4.867269 & -3.381657 & 2.188296 \\ 5.298617 & -4.324064 & 2.541588 \\ 3.912351 & -3.592605 & 1.697417 \\ 5.535880 & -2.978617 & 1.424757 \\ 7.127496 & -0.725931 & 2.188675 \\ 4.922872 & -0.687524 & 1.165658 \\ 3.938269 & -3.112997 & 4.529283 \\ 2.497477 & -3.448928 & 4.082129 \\ 3.910635 & -2.444978 & 5.398888 \\ 4.449555 & -4.026239 & 4.850536 \\ 1.881040 & -3.615036 & 4.972653 \\ 2.493653 & -4.407379 & 3.542935 \\ 2.400273 & 0.411720 & 1.379724 \\ 0.824811 & -1.142827 & 0.378234 \\ 3.881379 & -0.567484 & 4.017002\end{array}$

\section{Structure: ${ }^{5}$ TS5}

C

C

C

C

$\mathrm{N}$

$\mathrm{H}$

$\mathrm{H}$

C

$\begin{array}{rrr}-0.230920 & -2.436093 & -1.557634 \\ 0.609326 & -3.486686 & -2.090029 \\ 1.878627 & -2.987954 & -2.159152 \\ 1.834732 & -1.634725 & -1.647352 \\ 0.555085 & -1.354949 & -1.240439 \\ 0.271318 & -4.457437 & -2.423592 \\ 2.751605 & -3.480744 & -2.563994 \\ 2.042191 & 2.706008 & -0.822093\end{array}$


3.487869

3.960403

2. 814851

1.677925

4.069200

4.995021

$-3.247743$

$-4.391460$

$-3.919453$

$-2.474959$

$-2.105856$

$-5.425207$

$-4.498367$

$-2.274919$

$-2.338304$

$-1.064945$

$-0.214834$

$-0.989923$

$-3.230017$

$-0.729212$

$-1.632141$

1.194354

2.899302

$-3.338854$

$-0.227333$

$-0.337558$

$-4.690897$

$-5.347666$

$-5.370736$

$-6.628725$

$-6.646041$

$-7.280807$

$-2.316955$

$-2.895620$

$-2.427327$

$-3.566094$

$-3.082477$

$-3.656041$

4.255040

5.077437

4.771957

6.360463

6.051196

6.851469
2.786005

1.542559

0.670843

1.410932

3.681073

1.244349

0.716299

$-0.157213$

$-1.408035$

$-1.329510$

$-0.026304$

0.152137

$-2.302036$

3. 006482

4.378835

4.859145

3.780069

2.708555

4.894968

5.838164

$-2.443241$

3. 791292

$-0.715089$

2.085123

0.743927

0.866379

2. 563153

2.000969

3.570192

2. 388142

3. 991591

3. 393080

$-3.756326$

$-4.433156$

$-4.348931$

$-5.643101$

$-5.565556$

$-6.211015$

$-1.269655$

$-0.824015$

$-2.282479$

$-1.335197$

$-2.799945$

$-2.324974$
$-0.854174$

$-1.142630$

$-1.307520$

$-1.108095$

$-0.687891$

$-1.227961$

$-0.835972$

$-0.990638$

$-1.245539$

$-1.256739$

$-1.027193$

$-0.937856$

$-1.427054$

$-0.409142$

0.076148

0.059653

$-0.429208$

$-0.724768$

0.402941

0.371595

$-1.474513$

$-0.504736$

$-1.598064$

$-0.487194$

$-1.101181$

$-3.408574$

$-0.081006$

1.021132

$-0.774614$

1.398756

$-0.400598$

0.686796

$-1.678556$

$-0.600990$

$-2.939092$

$-0.758502$

$-3.122950$

$-2.028792$

$-1.864157$

$-2.906314$

$-1.051154$

$-3.100175$

$-1.211560$

$-2.248966$ 
C

C

C

C

C

C

F

F

$\mathrm{F}$

F

F

F

$\mathrm{F}$

F

$\mathrm{F}$

F

F

F

F

F

F

F

F

$\mathrm{F}$

F

$\mathrm{F}$

O

C

C

C

C

C

C

$\mathrm{H}$

C

C

$\mathrm{H}$

$\mathrm{H}$

C

C

O

C

$\mathrm{H}$

$\mathrm{H}$
1.858551

1.724631

2. 614956

2. 318237

3. 223844

3. 071610

$-4.752851$

$-7.232052$

$-8.507587$

$-7.270655$

$-4.800929$

1.006578

2.174196

3. 647253

3. 943810

2.773852

4.647746

7.123571

8.081779

6.513644

4. 026114

$-2.821923$

$-4.113033$

$-4.289540$

$-3.169594$

$-1.887713$

$-0.068136$

$-2.776724$

$-1.880546$

$-0.512229$

$-0.018284$

$-0.976989$

$-2.330153$

$-2.232981$

0.430200

1. 375802

$-0.648798$

$-3.044800$

2. 272778

1.805502

$-4.096622$

$-6.357901$

$-7.224349$

$-6.481488$
5.079046

6.215196

5.209588

7.430027

6.410388

7.525523

1.039052

1.798151

3.779755

4.953923

4.153050

6.153856

8.500022

8.683457

6.503376

4.156114

0.126282

$-0.884568$

$-2.810184$

$-3.732486$

$-2.765287$

$-3.907189$

$-6.261551$

$-7.374277$

$-6.113383$

$-3.751168$

0.640656

$-1.910473$

$-1.093091$

$-1.259908$

$-2.220365$

$-3.063541$

$-2.928495$

$-0.317239$

$-0.455849$

$-2.235408$

$-3.836100$

$-3.583348$

$-1.343252$

$-0.459452$

$-1.598462$

$-1.826586$

$-2.467843$

$-0.857950$
$-0.146551$

$-0.951091$

1.022805

$-0.613337$

1. 377455

0.555186

1.743860

2.441402

1.043053

$-1.086714$

$-1.835197$

$-2.077948$

$-1.401853$

0.887499

2. 501831

1.838437

$-3.741019$

$-4.099959$

$-2.424244$

$-0.366704$

$-0.039099$

0.631947

0.291937

$-2.197030$

$-4.339528$

$-4.007755$

0.835774

2. 726512

2.064023

2. 259215

3.194325

3.830371

3.600813

1.400159

1. 452102

3. 521933

4.516038

4.078919

2. 881611

1.919348

2.499968

1. 946131

2.110563

2.437441 


$\begin{array}{lrrr}\mathrm{H} & -6.244543 & -1.629178 & 0.873603 \\ \mathrm{C} & -5.110877 & -2.525196 & 2.423349 \\ \mathrm{O} & -4.983319 & -3.697855 & 2.666837 \\ \mathrm{C} & 3.706363 & -1.290015 & 3.358249 \\ \mathrm{C} & 4.218615 & -2.657944 & 3.851761 \\ \mathrm{C} & 4.829503 & -0.750204 & 2.451908 \\ \mathrm{H} & 4.772768 & 0.333219 & 2.309520 \\ \mathrm{C} & 6.108979 & -1.182974 & 3.210982 \\ \mathrm{H} & 6.525435 & -0.373281 & 3.821284 \\ \mathrm{C} & 5.677129 & -2.318055 & 4.163320 \\ \mathrm{O} & 6.369806 & -2.840018 & 5.007494 \\ \mathrm{C} & 4.263525 & -3.752918 & 2.756906 \\ \mathrm{H} & 4.533670 & -4.707496 & 3.220337 \\ \mathrm{H} & 3.296464 & -3.865634 & 2.257136 \\ \mathrm{H} & 5.000218 & -3.543662 & 1.978390 \\ \mathrm{H} & 6.915728 & -1.529557 & 2.556271 \\ \mathrm{H} & 4.772656 & -1.213805 & 1.466193 \\ \mathrm{C} & 3.342330 & -3.086849 & 5.022591 \\ \mathrm{C} & 1.873920 & -3.230171 & 4.561472 \\ \mathrm{H} & 3.410528 & -2.334629 & 5.817842 \\ \mathrm{H} & 3.687626 & -4.031761 & 5.454394 \\ \mathrm{H} & 1.218432 & -3.184346 & 5.438351 \\ \mathrm{H} & 1.719127 & -4.232911 & 4.137588 \\ \mathrm{H} & 2.480861 & 0.224785 & 1.415045 \\ \mathrm{H} & 0.668072 & -1.226495 & 0.561232 \\ \mathrm{H} & 3.699993 & -0.640063 & 4.250716\end{array}$

\section{Structure: ${ }^{3}$ INT5}

C
C
C
C
N
H
H
C
C
C
C
N
H
H
C

$$
\begin{array}{rrr}
-1.821712 & 2.873050 & 0.729012 \\
-1.910701 & 4.252464 & 1.041000 \\
-0.697338 & 4.846857 & 0.747905 \\
0.177052 & 3.854506 & 0.239237 \\
-0.542337 & 2.650888 & 0.200971 \\
-2.761084 & 4.719778 & 1.517249 \\
-0.414222 & 5.870352 & 0.948867 \\
3.486939 & 0.974979 & 0.105953 \\
4.479195 & 1.906142 & -0.392376 \\
3.848479 & 3.095884 & -0.559390 \\
2.465474 & 2.909734 & -0.155850 \\
2.278717 & 1.602183 & 0.220381 \\
5.517805 & 1.675455 & -0.579980 \\
4.272257 & 4.020237 & -0.924184 \\
-1.514782 & -1.476365 & 1.368668
\end{array}
$$




\begin{tabular}{|c|c|c|c|}
\hline $\mathrm{C}$ & -2.935677 & -1.699895 & 1.527193 \\
\hline $\mathrm{C}$ & -3.543924 & -0.497149 & 1.371248 \\
\hline $\mathrm{C}$ & -2.503379 & 0.485595 & 1.129487 \\
\hline $\mathrm{N}$ & -1.281958 & -0.147763 & 1.131029 \\
\hline $\mathrm{H}$ & -3.403566 & -2.651757 & 1.729373 \\
\hline $\mathrm{H}$ & -4.603083 & -0.292239 & 1.410912 \\
\hline $\mathrm{C}$ & 0.846186 & -2.268344 & 1.294046 \\
\hline $\mathrm{C}$ & 1.861548 & -3.296261 & 1.363610 \\
\hline $\mathrm{C}$ & 3.056258 & -2.693796 & 1.126109 \\
\hline $\mathrm{C}$ & 2.790543 & -1.295843 & 0.877232 \\
\hline $\mathrm{N}$ & 1.442834 & -1.067796 & 1.002539 \\
\hline $\mathrm{H}$ & 1.684538 & -4.348116 & 1.526048 \\
\hline $\mathrm{H}$ & 4.027498 & -3.162946 & 1.085649 \\
\hline $\mathrm{C}$ & -2.762492 & 1.866967 & 0.991574 \\
\hline $\mathrm{C}$ & 3.742256 & -0.365577 & 0.435219 \\
\hline $\mathrm{C}$ & 1.534708 & 3.970112 & -0.081164 \\
\hline $\mathrm{C}$ & -0.533006 & -2.478381 & 1.446615 \\
\hline Mn & 0.549281 & 0.644753 & 0.758355 \\
\hline $\mathrm{Cl}$ & 0.769539 & 1.337456 & 2.908226 \\
\hline $\mathrm{C}$ & -0.987782 & -3.878534 & 1.688503 \\
\hline C & -1.726289 & -4.578952 & 0.730336 \\
\hline $\mathrm{C}$ & -0.677887 & -4.555958 & 2.872372 \\
\hline $\mathrm{C}$ & -2.146449 & -5.890145 & 0.929572 \\
\hline $\mathrm{C}$ & -1.079560 & -5.871720 & 3.093439 \\
\hline $\mathrm{C}$ & -1.817395 & -6.540464 & 2.117450 \\
\hline $\mathrm{C}$ & -4.161298 & 2.343499 & 1.169214 \\
\hline $\mathrm{C}$ & -4.817350 & 2.998836 & 0.119122 \\
\hline $\mathrm{C}$ & -4.876981 & 2.173707 & 2.360489 \\
\hline $\mathrm{C}$ & -6.135628 & 3.430822 & 0.223367 \\
\hline $\mathrm{C}$ & -6.196186 & 2.602629 & 2.490779 \\
\hline C & -6.826520 & 3.232633 & 1.418243 \\
\hline $\mathrm{C}$ & 2.034795 & 5.361136 & -0.257356 \\
\hline $\mathrm{C}$ & 2.996438 & 5.915115 & 0.597896 \\
\hline C & 1.524920 & 6.186581 & -1.267323 \\
\hline $\mathrm{C}$ & 3.444587 & 7.225336 & 0.447868 \\
\hline $\mathrm{C}$ & 1.955479 & 7.500269 & -1.432951 \\
\hline $\mathrm{C}$ & 2.920413 & 8.019599 & -0.571143 \\
\hline $\mathrm{C}$ & 5.125359 & -0.874888 & 0.207500 \\
\hline $\mathrm{C}$ & 5.990946 & -1.169499 & 1.263307 \\
\hline $\mathrm{C}$ & 5.581997 & -1.113308 & -1.091495 \\
\hline C & 7.267428 & -1.684006 & 1.040720 \\
\hline C & 6.851996 & -1.626552 & -1.336623 \\
\hline C & 7.696017 & -1.915518 & -0.266002 \\
\hline $\mathrm{F}$ & -2.061008 & -3.982275 & -0.422721 \\
\hline
\end{tabular}


C

C

$\mathrm{H}$

C

C

$\mathrm{H}$

$\mathrm{H}$

C

C

O

C

$\mathrm{H}$

$\mathrm{H}$

$\mathrm{H}$

C

O

C

C

C

$\mathrm{H}$

\begin{tabular}{|c|c|c|}
\hline-2.854483 & -6.523051 & -0.011472 \\
\hline-2.209591 & -7.798652 & 2.322243 \\
\hline-0.767321 & -6.493653 & 4.234094 \\
\hline & -3.940922 & \\
\hline & -0.969671 & 2.52474 \\
\hline 8. & -1.958906 & \\
\hline & -2.420603 & -0.4 \\
\hline & -1.8973 & -2.588 \\
\hline & -0.863098 & -2.13 \\
\hline 3 & 5.18 & \\
\hline & & \\
\hline & & -0.7 \\
\hline & & \\
\hline & & -2 . \\
\hline-1 & & -1 \\
\hline & & -0 \\
\hline 8 . & 3.6 & \\
\hline & & \\
\hline-4 . & & \\
\hline 0.2 & 0.4 & $-1 \cdot 1$ \\
\hline $1 . \varepsilon$ & -3.1 & -2 . \\
\hline & -1.9 & -2 \\
\hline-0. & -1.6 & -2.1 \\
\hline$-0 . \varepsilon$ & -2.6 & -2.7 \\
\hline-0 & -3.9 & -3 \\
\hline & -4.2 & \\
\hline 2.0 & $-1 \cdot 1$ & -1 \\
\hline-0.56 & -0.4 & -1 \\
\hline-2 & -2.3 & \\
\hline-0. & -4.7 & -3 \\
\hline 1.4 & -5.1 & -3.0 \\
\hline$-2 \cdot 7$ & $-1 \cdot 1$ & \\
\hline$-1 \cdot 9$ & -0.1 & -2 . \\
\hline 3.2 & -3.4 & $-2 \cdot 2$ \\
\hline 5.2 & -4.5527 & $-1.604^{7}$ \\
\hline 5.51 & -5.586777 & -1.7763 \\
\hline 5.6 & -3.89 & -2.372 \\
\hline 5.60 & -4.250078 & -0.629 \\
\hline 3.6 & -4.49 & -1 \\
\hline 2.99 & -5.295244 & -0.9764 \\
\hline-4.203531 & -0.797280 & -2.8822 \\
\hline-5.1104 & -2.033466 & -2.7099 \\
\hline & 0.326 & -2.123 \\
\hline-4.6 & 1.32 & -2 \\
\hline
\end{tabular}


C
H
C
O
C
H
H
H
H
H
C
C
H
H
H
H
H
H
H

\section{Structure: ${ }^{5}$ INT5}

C
C
C
C
H
H
C
C
C
C
N
H
H
C
C
C
C
N
H
H
C

$\begin{array}{rrr}-6.418617 & 0.062575 & -2.471717 \\ -6.757048 & 0.675459 & -3.315932 \\ -6.494442 & -1.417196 & -2.906654 \\ -7.483515 & -1.979712 & -3.323002 \\ -5.110384 & -2.602181 & -1.268434 \\ -5.771378 & -3.474574 & -1.225670 \\ -4.106565 & -2.900572 & -0.961420 \\ -5.473007 & -1.873187 & -0.536057 \\ -7.117613 & 0.252302 & -1.649625 \\ -4.755187 & 0.242544 & -1.046776 \\ -4.669247 & -3.088555 & -3.719098 \\ -3.194263 & -3.486826 & -3.469008 \\ -4.779918 & -2.674550 & -4.729180 \\ -5.312090 & -3.974384 & -3.671838 \\ -2.789696 & -3.913761 & -4.395472 \\ -3.162701 & -4.302431 & -2.732623 \\ -2.311358 & 0.800429 & -1.716265 \\ -0.463745 & 2.070257 & -0.660649 \\ -4.271096 & -0.525854 & -3.950670\end{array}$

-6.418617
-6.757048 .

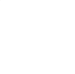

$-1.805780$

2.902747

0.654214

$-1.874319$

4.280109

1.008704

$-0.657471$

4.861629

0.720889

0.195531

3.861306

0.174756

$-0.548715$

2.690027

0.103052

$-2.709315$

4.740636

1.517349

$-0.347920$

5.869460

0.958004

3.475688

0.907996

0.165684

4.486978

1.828195

$-0.313633$

3.882784

3.030452

$-0.499338$

2.489940

2.875581

$-0.133317$

2.277152

1.571146

0.248528

5.527004

1.585051

$-0.474306$

4.334211

3.943065

$-0.860352$

$-1.496542$

$-1.457271$

1.381554

$-2.919647$

$-1.665233$

1.540183

$-3.529190$

$-0.463198$

1.369700

$-2.494936$

0.516792

1.112409

$-1.275164$

$-0.123845$

1.125480

$-3.392694$

$-2.610937$

1. 758219

$-4.588985$

$-0.260875$

1.405668

0.857446

$-2.342494$

1. 340403 


\begin{tabular}{|c|c|c|c|}
\hline C & 1.850727 & -3.405258 & 1.356070 \\
\hline C & 3.054652 & -2.819520 & 1.116983 \\
\hline C & 2.806302 & -1.400432 & 0.927936 \\
\hline $\mathrm{N}$ & 1.479097 & -1.157520 & 1.100764 \\
\hline $\mathrm{H}$ & 1.653818 & -4.459589 & 1.479720 \\
\hline $\mathrm{H}$ & 4.013648 & -3.309431 & 1.038507 \\
\hline C & -2.747496 & 1.896597 & 0.941488 \\
\hline C & 3.736298 & -0.442648 & 0.487744 \\
\hline $\mathrm{C}$ & 1.569252 & 3.948325 & -0.103594 \\
\hline $\mathrm{C}$ & -0.535505 & -2.488714 & 1.475953 \\
\hline Mn & 0.544876 & 0.667555 & 0.797185 \\
\hline $\mathrm{Cl}$ & 0.651375 & 1.565329 & 2.913647 \\
\hline $\mathrm{C}$ & -1.036691 & -3.875553 & 1.705694 \\
\hline C & -1.783741 & -4.553502 & 0.738214 \\
\hline C & -0.746122 & -4.570668 & 2.884466 \\
\hline C & -2.235350 & -5.855849 & 0.924111 \\
\hline C & -1.179402 & -5.878641 & 3.092986 \\
\hline C & -1.927546 & -6.522949 & 2.108418 \\
\hline C & -4.141293 & 2.382114 & 1.128741 \\
\hline $\mathrm{C}$ & -4.799326 & 3.043310 & 0.083028 \\
\hline $\mathrm{C}$ & -4.849574 & 2.223073 & 2.326519 \\
\hline $\mathrm{C}$ & -6.112041 & 3.489291 & 0.196934 \\
\hline $\mathrm{C}$ & -6.163068 & 2.666253 & 2.466208 \\
\hline C & -6.795459 & 3.300769 & 1.397599 \\
\hline C & 2.097545 & 5.329998 & -0.264842 \\
\hline C & 3.048031 & 5.868605 & 0.613112 \\
\hline C & 1.619960 & 6.166578 & -1.281980 \\
\hline C & 3.516520 & 7.173218 & 0.477268 \\
\hline $\mathrm{C}$ & 2.071640 & 7.474767 & -1.433505 \\
\hline C & 3.024860 & 7.978105 & -0.549575 \\
\hline C & 5.119144 & -0.941221 & 0.232586 \\
\hline C & 5.985268 & -1.285177 & 1.273261 \\
\hline $\mathrm{C}$ & 5.571861 & -1.132467 & -1.075988 \\
\hline C & 7.258675 & -1.796922 & 1.027186 \\
\hline $\mathrm{C}$ & 6.838698 & -1.641043 & -1.344624 \\
\hline C & 7.683805 & -1.976979 & -0.288593 \\
\hline $\mathrm{F}$ & -2.095128 & -3.941940 & -0.413997 \\
\hline $\mathrm{F}$ & -2.951104 & -6.464761 & -0.027285 \\
\hline $\mathrm{F}$ & -2.349049 & -7.773565 & 2.301261 \\
\hline $\mathrm{F}$ & -0.886914 & -6.516748 & 4.229938 \\
\hline $\mathrm{F}$ & -0.026851 & -3.980490 & 3.846262 \\
\hline $\mathrm{F}$ & 5.594044 & -1.133356 & 2.543117 \\
\hline $\mathrm{F}$ & 8.070707 & -2.117193 & 2.038695 \\
\hline $\mathrm{F}$ & 8.895054 & -2.478699 & -0.536645 \\
\hline
\end{tabular}




\begin{tabular}{|c|c|c|c|}
\hline $\mathrm{F}$ & 7.223888 & -1.865963 & -2.607025 \\
\hline $\mathrm{F}$ & 4.771975 & -0.840131 & -2.108119 \\
\hline $\mathrm{F}$ & 3.525928 & 5.130091 & 1.619766 \\
\hline $\mathrm{F}$ & 4.422252 & 7.661089 & 1.329737 \\
\hline $\mathrm{F}$ & 3.465076 & 9.229342 & -0.685468 \\
\hline $\mathrm{F}$ & 1.602180 & 8.246126 & -2.418665 \\
\hline $\mathrm{F}$ & 0.706276 & 5.710271 & -2.148675 \\
\hline $\mathrm{F}$ & -4.167296 & 3.242186 & -1.084770 \\
\hline $\mathrm{F}$ & -6.716406 & 4.092917 & -0.830308 \\
\hline $\mathrm{F}$ & -8.052126 & 3.726801 & 1.524201 \\
\hline $\mathrm{F}$ & -6.816163 & 2.497339 & 3.618945 \\
\hline $\mathrm{F}$ & -4.270526 & 1.628481 & 3.374757 \\
\hline O & 0.207466 & 0.504671 & -1.160257 \\
\hline C & 1.838946 & -3.188288 & -2.320666 \\
\hline C & 1.348301 & -1.945656 & -2.001900 \\
\hline $\mathrm{C}$ & -0.028361 & -1.664747 & -2.169893 \\
\hline C & -0.899926 & -2.652356 & -2.734803 \\
\hline C & -0.333987 & -3.921163 & -3.045421 \\
\hline C & 0.998767 & -4.195672 & -2.840604 \\
\hline $\mathrm{H}$ & 2.009011 & -1.174083 & -1.631357 \\
\hline C & -0.572771 & -0.398976 & -1.764993 \\
\hline $\mathrm{C}$ & -2.287063 & -2.363156 & -2.945318 \\
\hline $\mathrm{H}$ & -0.963073 & -4.702529 & -3.455157 \\
\hline $\mathrm{H}$ & 1.406835 & -5.170734 & -3.075643 \\
\hline $\mathrm{C}$ & -2.763894 & -1.098366 & -2.602931 \\
\hline C & -1.912797 & -0.138234 & -2.019246 \\
\hline 0 & 3.220673 & -3.390942 & -2.203865 \\
\hline $\mathrm{C}$ & 5.204227 & -4.555079 & -1.658013 \\
\hline $\mathrm{H}$ & 5.525731 & -5.583414 & -1.838957 \\
\hline $\mathrm{H}$ & 5.603726 & -3.886963 & -2.422195 \\
\hline $\mathrm{H}$ & 5.603742 & -4.252289 & -0.683215 \\
\hline C & 3.694823 & -4.517582 & -1.598184 \\
\hline O & 3.005363 & -5.354505 & -1.064261 \\
\hline C & -4.211879 & -0.775153 & -2.882926 \\
\hline $\mathrm{C}$ & -5.122473 & -2.007305 & -2.701474 \\
\hline $\mathrm{C}$ & -4.940482 & 0.357679 & -2.135687 \\
\hline $\mathrm{H}$ & -4.609740 & 1.350215 & -2.446626 \\
\hline C & -6.424961 & 0.094497 & -2.483692 \\
\hline $\mathrm{H}$ & -6.760703 & 0.701411 & -3.333253 \\
\hline C & -6.504289 & -1.388410 & -2.906464 \\
\hline O & -7.493551 & -1.952004 & -3.320580 \\
\hline $\mathrm{C}$ & -5.127257 & -2.563819 & -1.255267 \\
\hline $\mathrm{H}$ & -5.790962 & -3.433829 & -1.206883 \\
\hline $\mathrm{H}$ & -4.125458 & -2.862953 & -0.942770 \\
\hline
\end{tabular}




$\mathrm{H}$
$\mathrm{H}$
$\mathrm{H}$
$\mathrm{C}$
$\mathrm{C}$
$\mathrm{H}$
$\mathrm{H}$
$\mathrm{H}$
$\mathrm{H}$
$\mathrm{H}$
$\mathrm{H}$
$\mathrm{H}$

\section{Structure: ${ }^{3}$ TS6}

\begin{tabular}{|c|c|c|c|}
\hline $\mathrm{C}$ & -1.678393 & 2.829937 & 0.853758 \\
\hline $\mathrm{C}$ & -1.770751 & 4.239550 & 1.028734 \\
\hline $\mathrm{C}$ & -0.559966 & 4.789352 & 0.680762 \\
\hline $\mathrm{C}$ & 0.305725 & 3.729503 & 0.287619 \\
\hline $\mathrm{N}$ & -0.405848 & 2.527822 & 0.359957 \\
\hline $\mathrm{H}$ & -2.632458 & 4.758440 & 1.424091 \\
\hline $\mathrm{H}$ & -0.273733 & 5.829337 & 0.747786 \\
\hline $\mathrm{C}$ & 3.556618 & 0.815981 & 0.201431 \\
\hline $\mathrm{C}$ & 4.576032 & 1.731444 & -0.261190 \\
\hline C & 3.967242 & 2.928466 & -0.457190 \\
\hline $\mathrm{C}$ & 2.575365 & 2.766210 & -0.088644 \\
\hline $\mathrm{N}$ & 2.352505 & 1.463504 & 0.290348 \\
\hline $\mathrm{H}$ & 5.615392 & 1.484931 & -0.420593 \\
\hline $\mathrm{H}$ & 4.414563 & 3.842785 & -0.818422 \\
\hline $\mathrm{C}$ & -1.525749 & -1.509957 & 1.352203 \\
\hline $\mathrm{C}$ & -2.945610 & -1.694052 & 1.543038 \\
\hline $\mathrm{C}$ & -3.515642 & -0.463848 & 1.474323 \\
\hline $\mathrm{C}$ & -2.450116 & 0.490196 & 1.252173 \\
\hline $\mathrm{N}$ & -1.248032 & -0.176928 & 1.183118 \\
\hline $\mathrm{H}$ & -3.440242 & -2.640396 & 1.703270 \\
\hline $\mathrm{H}$ & -4.565246 & -0.224843 & 1.555315 \\
\hline C & 0.791498 & -2.377797 & 1.158070 \\
\hline C & 1.762224 & -3.446013 & 1.155096 \\
\hline $\mathrm{C}$ & 2.981771 & -2.876316 & 0.970394 \\
\hline $\mathrm{C}$ & 2.770668 & -1.457350 & 0.820831 \\
\hline $\mathrm{N}$ & 1.429246 & -1.179098 & 0.936835 \\
\hline $\mathrm{H}$ & 1.540391 & -4.498298 & 1.243501 \\
\hline $\mathrm{H}$ & 3.932587 & -3.380856 & 0.889859 \\
\hline $\mathrm{C}$ & -2.659698 & 1.879406 & 1.150048 \\
\hline
\end{tabular}

$\begin{array}{rrr}-5.489885 & -1.827793 & -0.529975 \\ -7.124258 & 0.293008 & -1.664002 \\ -4.762841 & 0.282956 & -1.057891 \\ -4.682749 & -3.071756 & -3.701346 \\ -3.208704 & -3.471109 & -3.447409 \\ -4.792196 & -2.666519 & -4.715068 \\ -5.326857 & -3.956139 & -3.646642 \\ -2.803804 & -3.905406 & -4.370251 \\ -3.178613 & -4.281160 & -2.705018 \\ -2.316635 & 0.826839 & -1.730136 \\ -0.419363 & 2.031153 & -0.694661 \\ -4.275704 & -0.513741 & -3.953995\end{array}$




\begin{tabular}{|c|c|c|c|}
\hline $\mathrm{C}$ & 3.772174 & -0.538156 & 0.487741 \\
\hline $\mathrm{C}$ & 1.657871 & 3.833333 & -0.050042 \\
\hline $\mathrm{C}$ & -0.585776 & -2.549963 & 1.337443 \\
\hline Mn & 0.608054 & 0.602974 & 0.884813 \\
\hline $\mathrm{Cl}$ & 0.919618 & 1.104186 & 3.028928 \\
\hline $\mathrm{C}$ & -1.088710 & -3.943737 & 1.510723 \\
\hline $\mathrm{C}$ & -1.859155 & -4.561485 & 0.522372 \\
\hline $\mathrm{C}$ & -0.802409 & -4.693713 & 2.655697 \\
\hline $\mathrm{C}$ & -2.335880 & -5.861511 & 0.655080 \\
\hline $\mathrm{C}$ & -1.259879 & -6.001017 & 2.810220 \\
\hline C & -2.030390 & -6.585941 & 1.805852 \\
\hline C & -4.039139 & 2.400961 & 1.348917 \\
\hline C & -4.690495 & 3.086095 & 0.315592 \\
\hline $\mathrm{C}$ & -4.746953 & 2.232774 & 2.544765 \\
\hline $\mathrm{C}$ & -5.996867 & 3.547631 & 0.438567 \\
\hline $\mathrm{C}$ & -6.054056 & 2.691465 & 2.694928 \\
\hline C & -6.680591 & 3.350031 & 1.637558 \\
\hline $\mathrm{C}$ & 2.163514 & 5.209899 & -0.312206 \\
\hline C & 3.111060 & 5.821385 & 0.517506 \\
\hline $\mathrm{C}$ & 1.676617 & 5.959394 & -1.389676 \\
\hline C & 3.568619 & 7.115464 & 0.280358 \\
\hline $\mathrm{C}$ & 2.115487 & 7.256556 & -1.642753 \\
\hline $\mathrm{C}$ & 3.066662 & 7.834640 & -0.803425 \\
\hline $\mathrm{C}$ & 5.163400 & -1.061307 & 0.375030 \\
\hline $\mathrm{C}$ & 5.895046 & -1.432583 & 1.507002 \\
\hline $\mathrm{C}$ & 5.776060 & -1.225485 & -0.868880 \\
\hline $\mathrm{C}$ & 7.185078 & -1.951003 & 1.408806 \\
\hline $\mathrm{C}$ & 7.062995 & -1.741133 & -0.991532 \\
\hline $\mathrm{C}$ & 7.769323 & -2.107729 & 0.152295 \\
\hline $\mathrm{F}$ & -2.170383 & -3.889368 & -0.595371 \\
\hline $\mathrm{F}$ & -3.074068 & -6.412858 & -0.313908 \\
\hline $\mathrm{F}$ & -2.474972 & -7.835424 & 1.947776 \\
\hline $\mathrm{F}$ & -0.969737 & -6.694877 & 3.914642 \\
\hline $\mathrm{F}$ & -0.063161 & -4.159908 & 3.635128 \\
\hline $\mathrm{F}$ & 5.352185 & -1.303908 & 2.721889 \\
\hline $\mathrm{F}$ & 7.862688 & -2.299412 & 2.506132 \\
\hline $\mathrm{F}$ & 9.000162 & -2.610990 & 0.044839 \\
\hline $\mathrm{F}$ & 7.604211 & -1.920991 & -2.202694 \\
\hline $\mathrm{F}$ & 5.113360 & -0.906600 & -1.989222 \\
\hline $\mathrm{F}$ & 3.601827 & 5.161539 & 1.572724 \\
\hline$F$ & 4.474914 & 7.674124 & 1.087828 \\
\hline $\mathrm{F}$ & 3.495528 & 9.075791 & -1.036409 \\
\hline F & 1.635493 & 7.946227 & -2.681992 \\
\hline $\mathrm{F}$ & 0.761673 & 5.429441 & -2.211700 \\
\hline
\end{tabular}




\begin{tabular}{|c|c|c|c|}
\hline $\mathrm{F}$ & -4.056828 & 3.296704 & -0.850221 \\
\hline $\mathrm{F}$ & -6.595047 & 4.172088 & -0.579914 \\
\hline $\mathrm{F}$ & -7.931769 & 3.789877 & 1.773033 \\
\hline $\mathrm{F}$ & -6.707336 & 2.511578 & 3.845973 \\
\hline F & -4.175040 & 1.607037 & 3.579302 \\
\hline O & 0.105103 & 0.720030 & -1.195733 \\
\hline $\mathrm{C}$ & 1.706968 & -2.875641 & -2.591158 \\
\hline $\mathrm{C}$ & 1.214054 & -1.660069 & -2.186438 \\
\hline $\mathrm{C}$ & -0.175567 & -1.395970 & -2.279100 \\
\hline $\mathrm{C}$ & -1.058189 & -2.374281 & -2.841838 \\
\hline C & -0.489703 & -3.622156 & -3.226308 \\
\hline C & 0.855956 & -3.878466 & -3.103086 \\
\hline $\mathrm{H}$ & 1.881427 & -0.897770 & -1.805678 \\
\hline $\mathrm{C}$ & -0.721427 & -0.159375 & -1.808054 \\
\hline $\mathrm{C}$ & -2.456237 & -2.095701 & -2.982991 \\
\hline $\mathrm{H}$ & -1.127718 & -4.399295 & -3.630021 \\
\hline $\mathrm{H}$ & 1.269009 & -4.835461 & -3.398392 \\
\hline $\mathrm{C}$ & -2.933959 & -0.855885 & -2.567255 \\
\hline $\mathrm{C}$ & -2.068612 & 0.097366 & -1.981263 \\
\hline O & 3.096600 & -3.051567 & -2.588308 \\
\hline $\mathrm{C}$ & 5.120044 & -4.250510 & -2.360888 \\
\hline $\mathrm{H}$ & 5.279229 & -5.020285 & -3.124623 \\
\hline $\mathrm{H}$ & 5.510879 & -3.303030 & -2.729541 \\
\hline $\mathrm{H}$ & 5.657123 & -4.567574 & -1.462858 \\
\hline $\mathrm{C}$ & 3.641444 & -4.182538 & -2.057292 \\
\hline 0 & 3.022480 & -5.021505 & -1.444652 \\
\hline $\mathrm{C}$ & -4.397381 & -0.544503 & -2.774705 \\
\hline $\mathrm{C}$ & -5.282268 & -1.799240 & -2.619294 \\
\hline $\mathrm{C}$ & -5.116475 & 0.538537 & -1.948073 \\
\hline $\mathrm{H}$ & -4.812929 & 1.549618 & -2.224099 \\
\hline $\mathrm{C}$ & -6.608366 & 0.267026 & -2.255018 \\
\hline $\mathrm{H}$ & -6.982513 & 0.906023 & -3.064098 \\
\hline $\mathrm{C}$ & -6.680491 & -1.195939 & -2.741790 \\
\hline O & -7.676361 & -1.757959 & -3.142319 \\
\hline $\mathrm{C}$ & -5.222888 & -2.424782 & -1.203045 \\
\hline $\mathrm{H}$ & -5.879101 & -3.301178 & -1.168974 \\
\hline $\mathrm{H}$ & -4.206920 & -2.731030 & -0.948567 \\
\hline $\mathrm{H}$ & -5.557956 & -1.726608 & -0.428825 \\
\hline $\mathrm{H}$ & -7.280962 & 0.417433 & -1.403409 \\
\hline $\mathrm{H}$ & -4.899047 & 0.415273 & -0.881891 \\
\hline $\mathrm{C}$ & -4.867327 & -2.806735 & -3.686130 \\
\hline $\mathrm{C}$ & -3.379031 & -3.192807 & -3.509251 \\
\hline $\mathrm{H}$ & -5.023330 & -2.354518 & -4.673559 \\
\hline $\mathrm{H}$ & -5.496186 & -3.703016 & -3.649923 \\
\hline
\end{tabular}


$\mathrm{H}$

$\mathrm{H}$

$\mathrm{H}$

$\mathrm{H}$

$\mathrm{H}$

$\begin{array}{rrr}-3.002338 & -3.567703 & -4.469421 \\ -3.309324 & -4.042271 & -2.815691 \\ -2.470652 & 1.044930 & -1.635966 \\ -0.351295 & 1.815394 & -0.605584 \\ -4.506666 & -0.232699 & -3.828544\end{array}$

\section{Structure: ${ }^{5}$ TS6}

\begin{tabular}{|c|c|c|c|}
\hline $\mathrm{C}$ & -1.697857 & 2.870287 & 0.728194 \\
\hline C & -1.778647 & 4.281035 & 0.958607 \\
\hline $\mathrm{C}$ & -0.569425 & 4.827415 & 0.610757 \\
\hline C & 0.274213 & 3.759677 & 0.164943 \\
\hline $\mathrm{N}$ & -0.456452 & 2.587229 & 0.191383 \\
\hline $\mathrm{H}$ & -2.621920 & 4.788914 & 1.404701 \\
\hline $\mathrm{H}$ & -0.258883 & 5.856091 & 0.726278 \\
\hline C & 3.534783 & 0.786743 & 0.226963 \\
\hline $\mathrm{C}$ & 4.549508 & 1.697458 & -0.247231 \\
\hline $\mathrm{C}$ & 3.946486 & 2.895188 & -0.464855 \\
\hline C & 2.553903 & 2.753771 & -0.10723 \\
\hline $\mathrm{N}$ & 2.332637 & 1.452417 & 0.29990 \\
\hline $\mathrm{H}$ & 5.588647 & 1.447343 & -0.40111 \\
\hline $\mathrm{H}$ & 4.401148 & 3.798537 & -0.84370 \\
\hline C & -1.479380 & -1.496247 & 1.35874 \\
\hline $\mathrm{C}$ & -2.899964 & -1.669421 & 1.5371 \\
\hline $\mathrm{C}$ & -3.474933 & -0.441785 & 1.44394 \\
\hline $\mathrm{C}$ & -2.421426 & 0.517238 & 1.213827 \\
\hline $\mathrm{N}$ & -1.213661 & -0.156038 & 1.174411 \\
\hline $\mathrm{H}$ & -3.397044 & -2.612472 & 1.70719 \\
\hline $\mathrm{H}$ & -4.527322 & -0.209864 & 1.50548 \\
\hline $\mathrm{C}$ & 0.829545 & -2.432842 & 1.20119 \\
\hline $\mathrm{C}$ & 1.795739 & -3.512729 & 1.17630 \\
\hline C & 3.016975 & -2.943577 & 0.98879 \\
\hline C & 2.803758 & -1.516986 & 0.86298 \\
\hline $\mathrm{N}$ & 1.474851 & -1.245157 & 1.01056 \\
\hline $\mathrm{H}$ & 1.571139 & -4.566103 & 1.24823 \\
\hline $\mathrm{H}$ & 3.967223 & -3.448676 & 0.89780 \\
\hline $\mathrm{C}$ & -2.652823 & 1.901970 & 1.07249 \\
\hline $\mathrm{C}$ & 3.775494 & -0.568452 & 0.51764 \\
\hline C & 1.645281 & 3.832248 & -0.119491 \\
\hline $\mathrm{C}$ & -0.556552 & -2.560233 & 1.36537 \\
\hline Mn & 0.610361 & 0.624027 & 0.90424 \\
\hline $\mathrm{Cl}$ & 0.739627 & 1.509626 & 3.02149 \\
\hline $\mathrm{C}$ & -1.091950 & -3.942531 & 1.53316 \\
\hline C & -1.878031 & -4.543185 & 0.54606 \\
\hline
\end{tabular}




\begin{tabular}{|c|c|c|c|}
\hline $\mathrm{C}$ & -0.804249 & -4.708134 & 2.667899 \\
\hline $\mathrm{C}$ & -2.369729 & -5.838167 & 0.670919 \\
\hline $\mathrm{C}$ & -1.276618 & -6.011093 & 2.814498 \\
\hline $\mathrm{C}$ & -2.063150 & -6.577388 & 1.811947 \\
\hline C & -4.030128 & 2.409040 & 1.314227 \\
\hline C & -4.715763 & 3.101969 & 0.307919 \\
\hline $\mathrm{C}$ & -4.697221 & 2.235669 & 2.533659 \\
\hline C & -6.016302 & 3.564843 & 0.479093 \\
\hline C & -5.997405 & 2.695685 & 2.731150 \\
\hline $\mathrm{C}$ & -6.658616 & 3.361529 & 1.699782 \\
\hline $\mathrm{C}$ & 2.183837 & 5.199649 & -0.359218 \\
\hline C & 3.124374 & 5.788421 & 0.495857 \\
\hline $\mathrm{C}$ & 1.725600 & 5.970651 & -1.434713 \\
\hline C & 3.602607 & 7.079282 & 0.283673 \\
\hline $\mathrm{C}$ & 2.186228 & 7.264760 & -1.662853 \\
\hline $\mathrm{C}$ & 3.129621 & 7.819207 & -0.799241 \\
\hline $\mathrm{C}$ & 5.172370 & -1.069761 & 0.373472 \\
\hline C & 5.936731 & -1.420846 & 1.490009 \\
\hline C & 5.750197 & -1.251058 & -0.884874 \\
\hline C & 7.226919 & -1.933240 & 1.363669 \\
\hline C & 7.035779 & -1.762288 & -1.035527 \\
\hline C & 7.776194 & -2.106792 & 0.093612 \\
\hline F & -2.190393 & -3.858745 & -0.563888 \\
\hline $\mathrm{F}$ & -3.122220 & -6.370993 & -0.297575 \\
\hline $\mathrm{F}$ & -2.521784 & -7.822500 & 1.946132 \\
\hline F & -0.985146 & -6.718470 & 3.909861 \\
\hline F & -0.049816 & -4.193813 & 3.646249 \\
\hline $\mathrm{F}$ & 5.427143 & -1.276207 & 2.717493 \\
\hline $\mathrm{F}$ & 7.937355 & -2.260802 & 2.446531 \\
\hline $\mathrm{F}$ & 9.005902 & -2.606222 & -0.040804 \\
\hline F & 7.541275 & -1.964285 & -2.258501 \\
\hline F & 5.054091 & -0.955714 & -1.990550 \\
\hline $\mathrm{F}$ & 3.586166 & 5.110988 & 1.552335 \\
\hline F & 4.500634 & 7.615819 & 1.114954 \\
\hline $\mathrm{F}$ & 3.578785 & 9.057342 & -1.008290 \\
\hline F & 1.734306 & 7.974022 & -2.701496 \\
\hline F & 0.820174 & 5.464037 & -2.281513 \\
\hline $\mathrm{F}$ & -4.123052 & 3.320431 & -0.877365 \\
\hline F & -6.648156 & 4.197984 & -0.513426 \\
\hline F & -7.903293 & 3.803096 & 1.881006 \\
\hline $\mathrm{F}$ & -6.610654 & 2.511486 & 3.903281 \\
\hline $\mathrm{F}$ & -4.091063 & 1.607463 & 3.546249 \\
\hline O & 0.111339 & 0.677502 & -1.201949 \\
\hline $\mathrm{C}$ & 1.682070 & -2.959775 & -2.516888 \\
\hline
\end{tabular}




\begin{tabular}{|c|c|c|c|}
\hline $\mathrm{C}$ & 1.200545 & -1.730343 & -2.138090 \\
\hline $\mathrm{C}$ & -0.180907 & -1.443596 & -2.264625 \\
\hline $\mathrm{C}$ & -1.069219 & -2.415514 & -2.829102 \\
\hline $\mathrm{C}$ & -0.512451 & -3.673834 & -3.194420 \\
\hline C & 0.826380 & -3.951423 & -3.042609 \\
\hline $\mathrm{H}$ & 1.873187 & -0.976752 & -1.749350 \\
\hline C & -0.715388 & -0.192245 & -1.817388 \\
\hline C & -2.462657 & -2.122664 & -2.985086 \\
\hline $\mathrm{H}$ & -1.154299 & -4.444043 & -3.605198 \\
\hline $\mathrm{H}$ & 1.227882 & -4.917370 & -3.322281 \\
\hline C & -2.929716 & -0.871842 & -2.587557 \\
\hline $\mathrm{C}$ & -2.059682 & 0.078535 & -2.007250 \\
\hline O & 3.067498 & -3.154556 & -2.464572 \\
\hline $\mathrm{C}$ & 5.081881 & -4.348039 & -2.135004 \\
\hline $\mathrm{H}$ & 5.338027 & -5.246916 & -2.704561 \\
\hline $\mathrm{H}$ & 5.461116 & -3.465127 & -2.648298 \\
\hline $\mathrm{H}$ & 5.558209 & -4.442709 & -1.153742 \\
\hline C & 3.582552 & -4.306921 & -1.949935 \\
\hline O & 2.931654 & -5.174591 & -1.415796 \\
\hline $\mathrm{C}$ & -4.388916 & -0.549212 & -2.804966 \\
\hline $\mathrm{C}$ & -5.285995 & -1.792589 & -2.629585 \\
\hline $\mathrm{C}$ & -5.097828 & 0.554887 & -1.998023 \\
\hline $\mathrm{H}$ & -4.784053 & 1.558238 & -2.290218 \\
\hline $\mathrm{C}$ & -6.591980 & 0.292788 & -2.301837 \\
\hline $\mathrm{H}$ & -6.958863 & 0.921105 & -3.122523 \\
\hline $\mathrm{C}$ & -6.678363 & -1.177639 & -2.762994 \\
\hline O & -7.679343 & -1.737155 & -3.153954 \\
\hline $\mathrm{C}$ & -5.233485 & -2.395280 & -1.203140 \\
\hline $\mathrm{H}$ & -5.897145 & -3.265384 & -1.155568 \\
\hline $\mathrm{H}$ & -4.220543 & -2.705999 & -0.941918 \\
\hline $\mathrm{H}$ & -5.564549 & -1.681787 & -0.441071 \\
\hline $\mathrm{H}$ & -7.263910 & 0.465170 & -1.453911 \\
\hline $\mathrm{H}$ & -4.882606 & 0.448331 & -0.929570 \\
\hline C & -4.880320 & -2.821220 & -3.679764 \\
\hline $\mathrm{C}$ & -3.395295 & -3.217707 & -3.497296 \\
\hline $\mathrm{H}$ & -5.033099 & -2.384200 & -4.674484 \\
\hline $\mathrm{H}$ & -5.516785 & -3.711307 & -3.62822 \\
\hline $\mathrm{H}$ & -3.021620 & -3.609875 & -4.451742 \\
\hline $\mathrm{H}$ & -3.332972 & -4.057549 & -2.791318 \\
\hline $\mathrm{H}$ & -2.453674 & 1.035736 & -1.680499 \\
\hline $\mathrm{H}$ & -0.333562 & 1.764242 & -0.694193 \\
\hline & -4.492699 & -0.254984 & -3.86432 \\
\hline
\end{tabular}




\section{Structure: ${ }^{3}$ INT6}

\begin{tabular}{|c|c|c|c|}
\hline C & 1.947260 & -1.749128 & -1.123589 \\
\hline C & 3.386167 & -1.811961 & -1.226710 \\
\hline C & 3.824681 & -0.547419 & -1.469064 \\
\hline $\mathrm{C}$ & 2.663498 & 0.309964 & -1.475056 \\
\hline $\mathrm{N}$ & 1.536372 & -0.445497 & -1.249639 \\
\hline $\mathrm{H}$ & 3.982601 & -2.706209 & -1.125813 \\
\hline $\mathrm{H}$ & 4.844767 & -0.216383 & -1.599430 \\
\hline $\mathrm{C}$ & -0.383433 & 3.223892 & -0.690877 \\
\hline $\mathrm{C}$ & 0.462907 & 4.391982 & -0.752882 \\
\hline $\mathrm{C}$ & 1.680600 & 3.979459 & -1.202619 \\
\hline $\mathrm{C}$ & 1.607696 & 2.547067 & -1.362326 \\
\hline $\mathrm{N}$ & 0.333948 & 2.118892 & -1.071599 \\
\hline $\mathrm{H}$ & 0.148296 & 5.399586 & -0.520834 \\
\hline $\mathrm{H}$ & 2.555243 & 4.585416 & -1.390491 \\
\hline $\mathrm{C}$ & -2.351781 & -2.051162 & -1.338922 \\
\hline $\mathrm{C}$ & -2.421889 & -3.495892 & -1.361777 \\
\hline $\mathrm{C}$ & -1.159798 & -3.958960 & -1.163552 \\
\hline C & -0.295808 & -2.801370 & -1.069630 \\
\hline $\mathrm{N}$ & -1.046789 & -1.661513 & -1.170277 \\
\hline $\mathrm{H}$ & -3.323295 & -4.074230 & -1.500504 \\
\hline $\mathrm{H}$ & -0.829484 & -4.987345 & -1.124507 \\
\hline $\mathrm{C}$ & -3.390319 & 0.183186 & -1.132069 \\
\hline $\mathrm{C}$ & -4.532567 & 1.005187 & -0.802898 \\
\hline $\mathrm{C}$ & -4.052582 & 2.210286 & -0.400415 \\
\hline C & -2.614672 & 2.162778 & -0.548722 \\
\hline $\mathrm{N}$ & -2.243000 & 0.918136 & -0.988216 \\
\hline $\mathrm{H}$ & -5.561493 & 0.677236 & -0.823505 \\
\hline $\mathrm{H}$ & -4.618337 & 3.060555 & -0.050702 \\
\hline $\mathrm{C}$ & 1.099991 & -2.856320 & -0.990839 \\
\hline $\mathrm{C}$ & -1.740961 & 3.236113 & -0.341673 \\
\hline $\mathrm{C}$ & 2.704615 & 1.704954 & -1.578508 \\
\hline $\mathrm{C}$ & -3.456959 & -1.194841 & -1.367912 \\
\hline Mn & -0.371661 & 0.231134 & -1.198462 \\
\hline $\mathrm{Cl}$ & -0.480584 & 0.316544 & -3.473572 \\
\hline $\mathrm{C}$ & 4.163815 & 0.105158 & 1.952281 \\
\hline C & 5.134804 & -0.895167 & 2.056239 \\
\hline $\mathrm{C}$ & 4.759882 & -2.226561 & 2.248895 \\
\hline $\mathrm{C}$ & 3.409462 & -2.571782 & 2.325427 \\
\hline $\mathrm{C}$ & 2.419275 & -1.590038 & 2.224384 \\
\hline $\mathrm{C}$ & 2.830152 & -0.276144 & 2.058057 \\
\hline $\mathrm{H}$ & 4.447748 & 1.136513 & 1.777071 \\
\hline $\mathrm{H}$ & 6.184067 & -0.625903 & 1.975971 \\
\hline
\end{tabular}




\begin{tabular}{|c|c|c|}
\hline 5.521866 & -2.996145 & 2.332381 \\
\hline 3.107529 & -3.603630 & 2.471704 \\
\hline 1.37144 & -1.854749 & 2.257328 \\
\hline & 1.272856 & 1.964289 \\
\hline-0.20992 & 0.089738 & 1.165810 \\
\hline 1.5011 & -0.059382 & 2.254379 \\
\hline-2.5366 & -0.743597 & 1.473244 \\
\hline-1.7879 & 1.208791 & 2.945965 \\
\hline-0.9019 & -1.229648 & 3.472644 \\
\hline$-0.332 \varepsilon$ & -0.774 & 4.661 \\
\hline-0.9 & -2.59 & 3.15 \\
\hline 0.2588 & -1.697 & 5.525 \\
\hline-0.365 & 0.28 & 4242 \\
\hline-0.3 & -3.49 & 4.02 \\
\hline-1.3 & -2.92 & 2.22 \\
\hline 0.2 & -3.06 & \\
\hline 0.7 & $-1 \cdot 34$ & 6.4 \\
\hline-0.3 & -4.55 & 3.770 \\
\hline 0.9 & -4.05 & \\
\hline 1.09 & -3.66 & 7.13 \\
\hline 0.4 & -4.99 & 6.1 \\
\hline 1.9 & -4.28 & \\
\hline-2.25 & 4.46 & 0.31 \\
\hline-1.7 & 4.81 & 1.5 \\
\hline-3.19 & 5.31 & -0.2 \\
\hline-2.2 & 5.95 & \\
\hline-3.6 & 6.45 & 0.384374 \\
\hline-3.17 & 6.77 & 1.65 \\
\hline-0.8 & 4.04 & \\
\hline$-1.75^{\circ}$ & 6.25 & 3.471 \\
\hline-3.6150 & 7.86 & 2.282 \\
\hline-4.5 & 7.25 & -0.1 \\
\hline-3.6740 & 5.04 & -1.488 \\
\hline-4.8128 & -1.798772 & -1.494 \\
\hline-5.3569 & -2.591499 & -0.4778 \\
\hline-5.6069 & -1.560599 & -2.620257 \\
\hline-6.632930 & -3.140481 & -0.580518 \\
\hline-4.646745 & -2.845130 & 0.62772 \\
\hline-6.888665 & -2.094386 & -2.740670 \\
\hline-5.141642 & -0.798785 & -3.616063 \\
\hline-7.400708 & -2.887977 & -1.71604 \\
\hline-7.128735 & -3.899175 & 0.404126 \\
\hline-7.62694 & -1.854121 & -3.829584 \\
\hline & -3.40 & -1.8 \\
\hline
\end{tabular}




$\begin{array}{lrrr}C & 1.726722 & -4.191665 & -0.783875 \\ C & 1.619628 & -4.840132 & 0.447821 \\ C & 2.453426 & -4.847874 & -1.782275 \\ C & 2.228003 & -6.063943 & 0.703257 \\ \text { F } & 0.937274 & -4.261454 & 1.454207 \\ C & 3.065948 & -6.080303 & -1.557077 \\ \text { F } & 2.589856 & -4.285942 & -2.987582 \\ \text { C } & 2.953009 & -6.690352 & -0.308426 \\ \text { F } & 2.135645 & -6.623673 & 1.917021 \\ \text { F } & 3.759389 & -6.681049 & -2.528773 \\ \text { F } & 3.544295 & -7.865076 & -0.079892 \\ C & 4.039752 & 2.331844 & -1.794628 \\ C & 4.724689 & 2.970220 & -0.757968 \\ C & 4.677420 & 2.273420 & -3.037710 \\ C & 5.983479 & 3.535810 & -0.934010 \\ \text { F } & 4.170593 & 3.043658 & 0.471875 \\ C & 5.939074 & 2.831408 & -3.241905 \\ \text { F } & 4.081180 & 1.662702 & -4.064244 \\ C & 6.591612 & 3.466348 & -2.186599 \\ \text { F } & 6.611509 & 4.133774 & 0.085361 \\ \text { F } & 6.526005 & 2.763569 & -4.439855 \\ \text { F } & 7.799175 & 4.003417 & -2.372717\end{array}$

\section{Structure: ${ }^{5}$ INT6}

$\begin{array}{lrrr}\mathrm{C} & 1.877762 & -1.824393 & -1.137404 \\ \mathrm{C} & 3.312740 & -1.960531 & -1.193090 \\ \mathrm{C} & 3.822257 & -0.719280 & -1.416878 \\ \mathrm{C} & 2.707777 & 0.195251 & -1.458689 \\ \mathrm{~N} & 1.537228 & -0.501774 & -1.278695 \\ \mathrm{H} & 3.858913 & -2.883624 & -1.071312 \\ \mathrm{H} & 4.861386 & -0.439301 & -1.511008 \\ \mathrm{C} & -0.203457 & 3.242230 & -0.714001 \\ \mathrm{C} & 0.696735 & 4.367366 & -0.753014 \\ \mathrm{C} & 1.902299 & 3.900006 & -1.183975 \\ \mathrm{C} & 1.765570 & 2.475698 & -1.354654 \\ \mathrm{~N} & 0.466598 & 2.108246 & -1.095774 \\ \mathrm{H} & 0.426464 & 5.386637 & -0.516830 \\ \mathrm{H} & 2.807997 & 4.464867 & -1.351880 \\ \mathrm{C} & -2.432781 & -1.914372 & -1.390369 \\ \mathrm{C} & -2.567232 & -3.351680 & -1.422573 \\ \mathrm{C} & -1.327107 & -3.873894 & -1.225728 \\ \mathrm{C} & -0.412604 & -2.761003 & -1.123105 \\ \mathrm{~N} & -1.110994 & -1.585303 & -1.221806 \\ & & & \end{array}$




\begin{tabular}{|c|c|c|c|}
\hline $\mathrm{H}$ & -3.493471 & -3.887691 & -1.567518 \\
\hline $\mathrm{H}$ & -1.044852 & -4.916447 & -1.191824 \\
\hline $\mathrm{C}$ & -3.360648 & 0.359099 & -1.174774 \\
\hline $\mathrm{C}$ & -4.454829 & 1.228875 & -0.811228 \\
\hline $\mathrm{C}$ & -3.909824 & 2.404754 & -0.405459 \\
\hline C & -2.481034 & 2.291113 & -0.585566 \\
\hline $\mathrm{N}$ & -2.178430 & 1.038303 & -1.058043 \\
\hline $\mathrm{H}$ & -5.497730 & 0.948077 & -0.808946 \\
\hline $\mathrm{H}$ & -4.425772 & 3.274300 & -0.027392 \\
\hline $\mathrm{C}$ & 0.976231 & -2.888439 & -1.027335 \\
\hline $\mathrm{C}$ & -1.559319 & 3.318838 & -0.369860 \\
\hline $\mathrm{C}$ & 2.826078 & 1.585458 & -1.551750 \\
\hline $\mathrm{C}$ & -3.499925 & -1.012402 & -1.410257 \\
\hline $\mathrm{Mn}$ & -0.339634 & 0.269943 & -1.334241 \\
\hline $\mathrm{Cl}$ & -0.398407 & 0.370773 & -3.730818 \\
\hline $\mathrm{C}$ & 4.125127 & -0.106548 & 2.028064 \\
\hline $\mathrm{C}$ & 5.032991 & -1.164836 & 2.128112 \\
\hline C & 4.575921 & -2.473801 & 2.295892 \\
\hline $\mathrm{C}$ & 3.206211 & -2.737787 & 2.349445 \\
\hline $\mathrm{C}$ & 2.278038 & -1.696901 & 2.248380 \\
\hline C & 2.769160 & -0.407170 & 2.109550 \\
\hline $\mathrm{H}$ & 4.473544 & 0.908830 & 1.875667 \\
\hline $\mathrm{H}$ & 6.097708 & -0.958290 & 2.066902 \\
\hline $\mathrm{H}$ & 5.288844 & -3.289074 & 2.379348 \\
\hline $\mathrm{H}$ & 2.841320 & -3.751022 & 2.479996 \\
\hline $\mathrm{H}$ & 1.215181 & -1.898733 & 2.263960 \\
\hline I & 1.357739 & 1.228821 & 2.018729 \\
\hline $\mathrm{N}$ & -0.255287 & 0.172844 & 1.211878 \\
\hline S & -1.533970 & 0.035707 & 2.298987 \\
\hline O & -2.600945 & -0.534413 & 1.464198 \\
\hline O & -1.757364 & 1.273534 & 3.066525 \\
\hline $\mathrm{C}$ & -1.042764 & -1.240361 & 3.458186 \\
\hline $\mathrm{C}$ & -0.434568 & -0.893124 & 4.664750 \\
\hline C & -1.165115 & -2.577799 & 3.070788 \\
\hline $\mathrm{C}$ & 0.077786 & -1.901686 & 5.481038 \\
\hline $\mathrm{H}$ & -0.372874 & 0.150413 & 4.955332 \\
\hline $\mathrm{C}$ & -0.651773 & -3.570586 & 3.901102 \\
\hline $\mathrm{H}$ & -1.642044 & -2.824881 & 2.127995 \\
\hline $\mathrm{C}$ & -0.014846 & -3.250130 & 5.109927 \\
\hline $\mathrm{H}$ & 0.559032 & -1.636797 & 6.419311 \\
\hline $\mathrm{H}$ & -0.723883 & -4.610232 & 3.594027 \\
\hline $\mathrm{C}$ & 0.532879 & -4.341091 & 5.998733 \\
\hline $\mathrm{H}$ & 1.331296 & -3.968590 & 6.648793 \\
\hline $\mathrm{H}$ & -0.253570 & -4.750742 & 6.646136 \\
\hline
\end{tabular}




\begin{tabular}{|c|c|c|c|}
\hline $\mathrm{H}$ & 0.932380 & -5.173094 & 5.408409 \\
\hline C & -2.015093 & 4.568330 & 0.296693 \\
\hline $\mathrm{C}$ & -1.536212 & 4.883825 & 1.573900 \\
\hline C & -2.909639 & 5.466310 & -0.291681 \\
\hline C & -1.934509 & 6.035329 & 2.246377 \\
\hline C & -3.328393 & 6.621970 & 0.364636 \\
\hline $\mathrm{C}$ & -2.836815 & 6.906388 & 1.637347 \\
\hline $\mathrm{F}$ & -0.659925 & 4.070157 & 2.183702 \\
\hline $\mathrm{F}$ & -1.459834 & 6.311452 & 3.465534 \\
\hline $\mathrm{F}$ & -3.227274 & 8.013799 & 2.271880 \\
\hline $\mathrm{F}$ & -4.187950 & 7.462060 & -0.220688 \\
\hline $\mathrm{F}$ & -3.393033 & 5.222502 & -1.516373 \\
\hline C & -4.883179 & -1.553038 & -1.510861 \\
\hline C & -5.437611 & -2.332808 & -0.489370 \\
\hline $\mathrm{C}$ & -5.693919 & -1.262280 & -2.612604 \\
\hline C & -6.740008 & -2.820355 & -0.565489 \\
\hline $\mathrm{F}$ & -4.712464 & -2.633105 & 0.594811 \\
\hline C & -7.001795 & -1.734361 & -2.705614 \\
\hline $\mathrm{F}$ & -5.219394 & -0.508239 & -3.609333 \\
\hline $\mathrm{C}$ & -7.523767 & -2.517286 & -1.677676 \\
\hline $\mathrm{F}$ & -7.245575 & -3.567788 & 0.422474 \\
\hline $\mathrm{F}$ & -7.755804 & -1.444887 & -3.771071 \\
\hline $\mathrm{F}$ & -8.774691 & -2.977379 & -1.757356 \\
\hline C & 1.532604 & -4.253938 & -0.817602 \\
\hline $\mathrm{C}$ & 1.370228 & -4.902517 & 0.407958 \\
\hline C & 2.247578 & -4.937737 & -1.805993 \\
\hline C & 1.914606 & -6.155019 & 0.667884 \\
\hline F & 0.695489 & -4.296649 & 1.402232 \\
\hline C & 2.795953 & -6.199234 & -1.576009 \\
\hline $\mathrm{F}$ & 2.435949 & -4.375359 & -3.003600 \\
\hline C & 2.629004 & -6.809760 & -0.333479 \\
\hline $\mathrm{F}$ & 1.770619 & -6.715875 & 1.875937 \\
\hline $\mathrm{F}$ & 3.479429 & -6.827251 & -2.537045 \\
\hline $\mathrm{F}$ & 3.158446 & -8.012519 & -0.101085 \\
\hline $\mathrm{C}$ & 4.196435 & 2.144259 & -1.726149 \\
\hline $\mathrm{C}$ & 4.883674 & 2.738601 & -0.665001 \\
\hline C & 4.865553 & 2.058597 & -2.951213 \\
\hline C & 6.175406 & 3.236901 & -0.800473 \\
\hline $\mathrm{F}$ & 4.298770 & 2.832067 & 0.548640 \\
\hline C & 6.160884 & 2.549156 & -3.114153 \\
\hline $\mathrm{F}$ & 4.267767 & 1.486505 & -3.998059 \\
\hline $\mathrm{C}$ & 6.815236 & 3.141978 & -2.035595 \\
\hline $\mathrm{F}$ & 6.804504 & 3.793299 & 0.241254 \\
\hline $\mathrm{F}$ & 6.778559 & 2.456031 & -4.294434 \\
\hline
\end{tabular}




\section{Structure: ${ }^{3} \mathrm{TS} 7$}

\begin{tabular}{|c|c|c|c|}
\hline $\mathrm{C}$ & 2.016569 & -1.668109 & -1.017018 \\
\hline $\mathrm{C}$ & 3.448425 & -1.657806 & -1.205825 \\
\hline C & 3.797988 & -0.381739 & -1.524317 \\
\hline $\mathrm{C}$ & 2.591769 & 0.410163 & -1.481782 \\
\hline $\mathrm{N}$ & 1.528438 & -0.397848 & -1.162026 \\
\hline $\mathrm{H}$ & 4.099456 & -2.513986 & -1.112325 \\
\hline $\mathrm{H}$ & 4.786651 & -0.001239 & -1.736491 \\
\hline $\mathrm{C}$ & -0.575636 & 3.155838 & -0.584455 \\
\hline $\mathrm{C}$ & 0.195078 & 4.370347 & -0.708818 \\
\hline $\mathrm{C}$ & 1.406829 & 4.019975 & -1.223737 \\
\hline C & 1.411702 & 2.582175 & -1.351537 \\
\hline $\mathrm{N}$ & 0.187543 & 2.087279 & -0.973557 \\
\hline $\mathrm{H}$ & -0.166998 & 5.362627 & -0.479903 \\
\hline $\mathrm{H}$ & 2.229267 & 4.672929 & -1.478344 \\
\hline C & -2.241305 & -2.200058 & -1.253455 \\
\hline $\mathrm{C}$ & -2.232511 & -3.647146 & -1.268946 \\
\hline $\mathrm{C}$ & -0.956997 & -4.040037 & -1.011799 \\
\hline $\mathrm{C}$ & -0.161696 & -2.835569 & -0.894543 \\
\hline $\mathrm{N}$ & -0.972569 & -1.739293 & -1.028535 \\
\hline $\mathrm{H}$ & -3.094239 & -4.274790 & -1.442120 \\
\hline $\mathrm{H}$ & -0.571873 & -5.048534 & -0.957183 \\
\hline $\mathrm{C}$ & -3.393788 & -0.027177 & -1.065138 \\
\hline $\mathrm{C}$ & -4.585134 & 0.738033 & -0.779210 \\
\hline C & -4.179810 & 1.957603 & -0.335634 \\
\hline $\mathrm{C}$ & -2.738038 & 1.977820 & -0.422320 \\
\hline $\mathrm{N}$ & -2.289509 & 0.753653 & -0.849836 \\
\hline $\mathrm{H}$ & -5.596656 & 0.366922 & -0.857327 \\
\hline $\mathrm{H}$ & -4.800207 & 2.777567 & -0.007611 \\
\hline C & 1.234098 & -2.814815 & -0.821345 \\
\hline $\mathrm{C}$ & -1.925114 & 3.096427 & -0.209653 \\
\hline $\mathrm{C}$ & 2.542214 & 1.801486 & -1.621944 \\
\hline $\mathrm{C}$ & -3.385913 & -1.400731 & -1.330689 \\
\hline $\mathrm{Mn}$ & -0.391227 & 0.169372 & -0.992791 \\
\hline $\mathrm{Cl}$ & -0.533313 & 0.231308 & -3.285393 \\
\hline $\mathrm{C}$ & 4.223406 & 0.351604 & 1.781197 \\
\hline $\mathrm{C}$ & 5.262946 & -0.576195 & 1.889268 \\
\hline C & 4.990358 & -1.913130 & 2.185658 \\
\hline C & 3.672124 & -2.335018 & 2.367956 \\
\hline $\mathrm{C}$ & 2.616168 & -1.424613 & 2.270937 \\
\hline $\mathrm{C}$ & 2.922495 & -0.099062 & 1.991534 \\
\hline
\end{tabular}


$\begin{array}{ll}4.429290 & 1.385037\end{array}$

$\begin{array}{ll}6.285623 & -0.246733\end{array}$

$5.805265-2.626555$

$3.446710-3.372642$

1.594702

1.339168

$-0.183393$

$-1.469268$

$-2.470194$

$-1.852037$

$-0.750571$

$-0.293067$

$-0.620406$

0.334471

$-0.437118$

0.011346

$-0.991390$

0.509074

0.695265

0.136005

1.244078

1.135263

0.882665

2.318350

$-2.494728$

$-2.015170$

$-3.486222$

$-2.510139$

$-4.001815$

$-3.509984$

$-1.043632$

$-2.033573$

$-3.992999$

$-4.953837$

$-3.971669$

$-4.702696$

$-5.269159$

$-5.439727$

$-6.510675$

$-4.614700$

$-6.686684$

$-4.952405$

$-7.221319$

$-7.028271$
$-1.754251$

1. 351451

$-0.042940$

$-0.179334$

$-1.040987$

1.133061

$-1.147053$

$-0.529663$

$-2.525521$

$-1.305171$

0.536917

$-3.283152$

$-2.985524$

$-2.685537$

$-0.830494$

$-4.351600$

$-3.506981$

$-3.083896$

$-4.540247$

$-3.541135$

4.304523

4.693579

5.101455

5.812058

6.221873

6.577925

3.987678

6.156797

7.652920

6.962531

4.795200

$-2.063108$

$-2.897265$

$-1.839794$

$-3.500652$

$-3.137184$

$-2.427749$

$-1.040187$

$-3.261850$

$-4.297790$
1.528149

1.729345

2.268761

2. 592947

2. 392111

1.908590

0.988493

2.112889

1. 475691

2. 659979

3.439382

4.602921

3.252676

5.577314

4.737875

4.235496

2. 343515

5.403418

6.486369

4.083065

6.435191

7.439190

6.458187

6.209365

0.444025

1.701170

$-0.134703$

2. 365273

0.513798

1.767931

2.299395

3.566693

2.395257

$-0.063546$

$-1.345545$

$-1.544546$

$-0.574662$

$-2.711752$

$-0.760902$

0.567900

$-2.915794$

$-3.666167$

$-1.935852$

0.181234 


\section{Structure: ${ }^{5}$ TS7}

-7.370319
-8.414325
1.937160
1.914853
2.652828
2.594440
1.246274
3.335754
2.707709
3.306733
2.580208
4.016263
3.965202
3.817272
4.535377
4.363950
5.740531
4.069695
5.570175
3.732330
6.257708
6.404277
6.070168
7.412483

$-2.200925$

$-4.042633$

$-3.832736-2.122209$

$-4.111767-0.611545$

$\begin{array}{ll}-4.741255 & 0.634040\end{array}$

$-4.751159-1.629064$

$\begin{array}{ll}-5.928601 & 0.883652\end{array}$

$-4.180088 \quad 1.659283$

$-5.946880-1.409895$

$-4.207646-2.849322$

$-6.537589-0.147246$

$-6.470107 \quad 2.108804$

$-6.531242-2.400605$

$-7.677103 \quad 0.075395$

$2.497895-1.955728$

$3.215617-0.996167$

$2.428699-3.241180$

$3.847060-1.287482$

$\begin{array}{ll}3.303094 & 0.267678\end{array}$

$3.051672-3.559757$

$1.744594-4.197847$

$3.765005-2.579502$

$4.521460-0.341233$

$2.971996-4.795995$

$4.365590-2.874776$
$-1.869743$

$-3.303209$

$-3.751888$

$-2.605432$

$-1.476018$

$-3.888569$

$-4.772971$

0.355178

$-0.505967$

$-1.695872$

$-1.597640$

$-0.330947$

$-0.217752$

$-2.569655$

2.428135

2.514120

1.262813
$1.824937-1.102825$

$1.913929-1.236783$

$0.668731-1.552804$

$-0.207225-1.560016$

$0.524364-1.290158$

$2.810849-1.102913$

$0.356672-1.71674$

$-3.151596-0.624715$

$-4.305254-0.706135$

$-3.881017-1.21775$

$-2.453644-1.390224$

$-2.044664-1.050631$

$-5.313823-0.44654$

$-4.477128-1.43709$

$2.057252-1.334234$

$3.497124-1.329938$

$3.974894-1.083751$ 


\begin{tabular}{|c|c|c|c|}
\hline $\mathrm{C}$ & 0.383398 & 2.834875 & -1.000693 \\
\hline $\mathrm{N}$ & 1.118986 & 1.686082 & -1.151247 \\
\hline $\mathrm{H}$ & 3.419311 & 4.066709 & -1.47929 \\
\hline $\mathrm{H}$ & 0.949408 & 5.006624 & -1.010043 \\
\hline C & 3.422848 & -0.185904 & -1.110251 \\
\hline C & 4.540702 & -1.022470 & -0.748973 \\
\hline C & 4.031814 & -2.203885 & -0.306913 \\
\hline C & 2.599991 & -2.135516 & -0.467049 \\
\hline $\mathrm{N}$ & 2.260432 & -0.894907 & -0.952192 \\
\hline $\mathrm{H}$ & 5.576517 & -0.717044 & -0.772994 \\
\hline $\mathrm{H}$ & 4.577471 & -3.051273 & 0.079078 \\
\hline C & -1.009538 & 2.910911 & -0.909579 \\
\hline C & 1.704231 & -3.186658 & -0.247222 \\
\hline C & -2.668812 & -1.599973 & -1.676905 \\
\hline $\mathrm{C}$ & 3.519280 & 1.184005 & -1.377706 \\
\hline Mn & 0.413352 & -0.182968 & -1.228223 \\
\hline $\mathrm{Cl}$ & 0.540713 & -0.318049 & -3.608005 \\
\hline $\mathrm{C}$ & -4.363163 & -0.078438 & 1.759334 \\
\hline C & -5.287817 & 0.969321 & 1.759297 \\
\hline $\mathrm{C}$ & -4.879061 & 2.273974 & 2.043155 \\
\hline $\mathrm{C}$ & -3.536529 & 2.539388 & 2.318109 \\
\hline $\mathrm{C}$ & -2.594067 & 1.506840 & 2.322965 \\
\hline $\mathrm{C}$ & -3.032559 & 0.212850 & 2.056129 \\
\hline $\mathrm{H}$ & -4.675112 & -1.089673 & 1.526559 \\
\hline $\mathrm{H}$ & -6.329171 & 0.757913 & 1.532533 \\
\hline $\mathrm{H}$ & -5.605309 & 3.081932 & 2.047635 \\
\hline $\mathrm{H}$ & -3.206230 & 3.548951 & 2.541553 \\
\hline $\mathrm{H}$ & -1.552353 & 1.720026 & 2.519535 \\
\hline I & -1.619760 & -1.399661 & 2.135141 \\
\hline N & 0.208996 & 0.057739 & 1.090997 \\
\hline S & 1.521648 & 0.079602 & 2.149678 \\
\hline O & 2.557096 & 0.881745 & 1.47354 \\
\hline O & 1.847127 & -1.261153 & 2.664411 \\
\hline C & 0.900634 & 1.071813 & 3.50349 \\
\hline $\mathrm{C}$ & 0.424436 & 0.467220 & 4.666328 \\
\hline $\mathrm{C}$ & 0.812348 & 2.454650 & 3.318872 \\
\hline C & -0.162363 & 1.263079 & 5.649426 \\
\hline $\mathrm{H}$ & 0.511501 & -0.607055 & 4.787675 \\
\hline $\mathrm{C}$ & 0.214962 & 3.232097 & 4.306763 \\
\hline $\mathrm{H}$ & 1.183968 & 2.902168 & 2.40392 \\
\hline C & -0.284129 & 2.650290 & 5.4827 \\
\hline $\mathrm{H}$ & -0.540043 & 0.798717 & 6.556896 \\
\hline $\mathrm{H}$ & 0.115738 & 4.303245 & 4.15219 \\
\hline C & -0.916446 & 3.506840 & 6.552997 \\
\hline
\end{tabular}




\begin{tabular}{|c|c|c|c|}
\hline $\mathrm{H}$ & -1.617521 & 2.932387 & 7.167143 \\
\hline $\mathrm{H}$ & -0.152081 & 3.918775 & 7.22503 \\
\hline $\mathrm{H}$ & -1.457242 & 4.354649 & 6.118593 \\
\hline C & 2.187435 & -4.422477 & 0.424115 \\
\hline C & 1.686791 & -4.768863 & 1.685373 \\
\hline C & 3.127602 & -5.283489 & -0.149317 \\
\hline $\mathrm{C}$ & 2.114031 & -5.910496 & 2.357853 \\
\hline C & 3.574492 & -6.427821 & 0.507701 \\
\hline $\mathrm{C}$ & 3.063768 & -6.741464 & 1.765795 \\
\hline $\mathrm{F}$ & 0.762611 & -4.000786 & 2.278177 \\
\hline $\mathrm{F}$ & 1.619883 & -6.215202 & 3.562505 \\
\hline $\mathrm{F}$ & 3.480933 & -7.838355 & 2.401649 \\
\hline $\mathrm{F}$ & 4.478466 & -7.230088 & -0.064059 \\
\hline $\mathrm{F}$ & 3.628734 & -5.015030 & -1.362457 \\
\hline $\mathrm{C}$ & 4.884716 & 1.752475 & -1.546588 \\
\hline $\mathrm{C}$ & 5.475508 & 2.553797 & -0.563355 \\
\hline $\mathrm{C}$ & 5.644314 & 1.462927 & -2.684903 \\
\hline C & 6.762965 & 3.064548 & -0.711174 \\
\hline $\mathrm{F}$ & 4.801400 & 2.851811 & 0.553324 \\
\hline $\mathrm{C}$ & 6.936475 & 1.957955 & -2.84967 \\
\hline $\mathrm{F}$ & 5.134027 & 0.688961 & -3.647921 \\
\hline $\mathrm{C}$ & 7.495013 & 2.762499 & -1.858236 \\
\hline $\mathrm{F}$ & 7.303531 & 3.832293 & 0.241866 \\
\hline $\mathrm{F}$ & 7.640834 & 1.669616 & -3.94877 \\
\hline $\mathrm{F}$ & 8.731512 & 3.243803 & -2.006508 \\
\hline $\mathrm{C}$ & -1.618586 & 4.241928 & -0.63431 \\
\hline $\mathrm{C}$ & -1.558668 & 4.793377 & 0.646577 \\
\hline C & -2.293412 & 4.978352 & -1.612468 \\
\hline $\mathrm{C}$ & -2.164411 & 6.003786 & 0.964856 \\
\hline $\mathrm{F}$ & -0.934103 & 4.129005 & 1.635262 \\
\hline C & -2.898898 & 6.200887 & -1.323738 \\
\hline F & -2.386117 & 4.503641 & -2.858803 \\
\hline $\mathrm{C}$ & -2.834950 & 6.714265 & -0.028729 \\
\hline $\mathrm{F}$ & -2.124265 & 6.467784 & 2.220724 \\
\hline $\mathrm{F}$ & -3.540758 & 6.882956 & -2.276693 \\
\hline $\mathrm{F}$ & -3.421746 & 7.877454 & 0.259895 \\
\hline $\mathrm{C}$ & -3.993935 & -2.197348 & -2.004708 \\
\hline $\mathrm{C}$ & -4.764921 & -2.866403 & -1.050393 \\
\hline $\mathrm{C}$ & -4.536308 & -2.071225 & -3.288528 \\
\hline $\mathrm{C}$ & -6.016372 & -3.397881 & -1.347644 \\
\hline $\mathrm{F}$ & -4.307898 & -3.005288 & 0.209459 \\
\hline $\mathrm{C}$ & -5.787692 & -2.594236 & -3.611588 \\
\hline F & -3.854567 & -1.427753 & -4.238743 \\
\hline C & -6.527729 & -3.261698 & -2.637239 \\
\hline
\end{tabular}




$\begin{array}{llll}F & -6.730023 & -4.027190 & -0.407076 \\ F & -6.281146 & -2.461461 & -4.845734 \\ F & -7.726432 & -3.766195 & -2.935882\end{array}$

\section{Structure: ${ }^{3}$ INT7}

$\begin{array}{lrrr}\text { C } & 2.508110 & -1.371885 & -0.998316 \\ \mathrm{C} & 3.921071 & -1.099328 & -0.955771 \\ \mathrm{C} & 4.068654 & 0.211446 & -0.617555 \\ \mathrm{C} & 2.746360 & 0.771065 & -0.500938 \\ \mathrm{~N} & 1.818524 & -0.216521 & -0.729136 \\ \mathrm{H} & 4.697096 & -1.830159 & -1.130162 \\ \mathrm{H} & 4.988504 & 0.764965 & -0.496138 \\ \mathrm{C} & -0.960646 & 2.884676 & -0.920373 \\ \mathrm{C} & -0.406405 & 4.216277 & -0.895907 \\ \mathrm{C} & 0.911775 & 4.095786 & -0.583177 \\ \mathrm{C} & 1.197234 & 2.685348 & -0.486438 \\ \mathrm{~N} & 0.040730 & 1.975318 & -0.689638 \\ \mathrm{H} & -0.963276 & 5.125906 & -1.064843 \\ \mathrm{H} & 1.634216 & 4.888298 & -0.456905 \\ \mathrm{C} & -1.495081 & -2.708594 & -0.245453 \\ \mathrm{C} & -1.217100 & -4.119183 & -0.322463 \\ \mathrm{C} & 0.058137 & -4.253926 & -0.775254 \\ \mathrm{C} & 0.608440 & -2.929103 & -0.904145 \\ \mathrm{~N} & -0.364849 & -2.007505 & -0.595375 \\ \mathrm{H} & -1.918553 & -4.905057 & -0.088159 \\ \mathrm{H} & 0.593324 & -5.170462 & -0.973450 \\ \mathrm{C} & -3.041939 & -0.798749 & -0.170234 \\ \mathrm{C} & -4.372611 & -0.245195 & -0.180927 \\ \mathrm{C} & -4.262913 & 1.051189 & -0.581753 \\ \mathrm{C} & -2.858836 & 1.322039 & -0.766734 \\ \mathrm{~N} & -2.144516 & 0.176181 & -0.523250 \\ \mathrm{H} & -5.273215 & -0.795206 & 0.050446 \\ \mathrm{H} & -5.054695 & 1.774692 & -0.711436 \\ \mathrm{C} & 1.950775 & -2.643400 & -1.151918 \\ \mathrm{C} & -2.322676 & 2.587879 & -1.019434 \\ \mathrm{C} & 2.471198 & 2.131233 & -0.328654 \\ \mathrm{C} & -2.743896 & -2.148875 & 0.032533 \\ \mathrm{Mn} & -0.158074 & -0.027428 & -0.632834 \\ \mathrm{C} 1 & -0.293959 & -0.059257 & -2.940369 \\ \mathrm{~N} & -0.084554 & 0.101882 & 1.227868 \\ \mathrm{~S} & 0.407549 & -1.101260 & 2.288505 \\ \mathrm{O} & -0.826516 & -1.840903 & 2.613045 \\ \mathrm{O} & 1.605964 & -1.825215 & 1.841645 \\ & & & \\ \mathrm{~N} & & \end{array}$




\begin{tabular}{|c|c|c|c|}
\hline C & 0.873896 & -0.088570 & 3.679706 \\
\hline $\mathrm{C}$ & -0.079468 & 0.247640 & 4.642295 \\
\hline $\mathrm{C}$ & 2.177420 & 0.406001 & 3.738605 \\
\hline $\mathrm{C}$ & 0.293974 & 1.089480 & 5.686736 \\
\hline $\mathrm{H}$ & -1.085998 & -0.150818 & 4.568815 \\
\hline C & 2.525680 & 1.260424 & 4.781458 \\
\hline $\mathrm{H}$ & 2.897523 & 0.131551 & 2.977072 \\
\hline $\mathrm{C}$ & 1.595142 & 1.610535 & 5.770560 \\
\hline $\mathrm{H}$ & -0.436880 & 1.353354 & 6.447101 \\
\hline $\mathrm{H}$ & 3.533107 & 1.666107 & 4.820467 \\
\hline $\mathrm{C}$ & 1.991236 & 2.508421 & 6.917794 \\
\hline $\mathrm{H}$ & 1.143030 & 3.101700 & 7.275314 \\
\hline $\mathrm{H}$ & 2.356824 & 1.916299 & 7.767287 \\
\hline $\mathrm{H}$ & 2.793461 & 3.196188 & 6.630635 \\
\hline $\mathrm{C}$ & 2.878615 & -3.767067 & -1.459441 \\
\hline $\mathrm{C}$ & 3.465724 & -3.883692 & -2.723254 \\
\hline $\mathrm{C}$ & 3.225777 & -4.718423 & -0.494306 \\
\hline C & 4.357244 & -4.910890 & -3.025458 \\
\hline C & 4.109497 & -5.757756 & -0.777555 \\
\hline $\mathrm{C}$ & 4.677093 & -5.850968 & -2.047401 \\
\hline $\mathrm{F}$ & 5.527827 & -6.840715 & -2.326408 \\
\hline $\mathrm{F}$ & 4.904445 & -5.001314 & -4.241806 \\
\hline $\mathrm{F}$ & 3.179678 & -2.989158 & -3.675616 \\
\hline $\mathrm{F}$ & 4.423015 & -6.658320 & 0.159868 \\
\hline $\mathrm{F}$ & 2.701575 & -4.647751 & 0.733448 \\
\hline $\mathrm{C}$ & -3.836783 & -3.026837 & 0.532048 \\
\hline $\mathrm{C}$ & -4.428559 & -4.016735 & -0.257750 \\
\hline $\mathrm{C}$ & -4.323995 & -2.864979 & 1.834780 \\
\hline $\mathrm{C}$ & -5.448172 & -4.832550 & 0.226955 \\
\hline $\mathrm{F}$ & -4.011060 & -4.205978 & -1.517352 \\
\hline $\mathrm{C}$ & -5.347352 & -3.665544 & 2.337510 \\
\hline F & -3.815806 & -1.916355 & 2.626311 \\
\hline C & -5.908454 & -4.653632 & 1.530301 \\
\hline $\mathrm{F}$ & -5.992386 & -5.775099 & -0.549781 \\
\hline $\mathrm{F}$ & -5.794279 & -3.492066 & 3.585516 \\
\hline $\mathrm{F}$ & -6.888180 & -5.426208 & 2.003795 \\
\hline $\mathrm{C}$ & -3.278573 & 3.693700 & -1.309176 \\
\hline $\mathrm{C}$ & -3.964561 & 3.740373 & -2.527438 \\
\hline $\mathrm{C}$ & -3.549572 & 4.701696 & -0.379095 \\
\hline $\mathrm{C}$ & -4.877152 & 4.753097 & -2.816550 \\
\hline $\mathrm{F}$ & -3.754386 & 2.793342 & -3.446938 \\
\hline $\mathrm{C}$ & -4.451946 & 5.727694 & -0.648879 \\
\hline $\mathrm{F}$ & -2.929033 & 4.698700 & 0.808940 \\
\hline C & -5.118436 & 5.750335 & -1.873064 \\
\hline
\end{tabular}




$\begin{array}{lrrr}\text { F } & -5.518187 & 4.776173 & -3.988790 \\ F & -4.688723 & 6.681608 & 0.257969 \\ F & -5.987747 & 6.726547 & -2.140955 \\ C & 3.623501 & 3.041556 & -0.072764 \\ C & 4.300496 & 3.021080 & 1.150268 \\ C & 4.093513 & 3.930899 & -1.045091 \\ C & 5.388005 & 3.850867 & 1.408897 \\ \text { F } & 3.908539 & 2.179904 & 2.119393 \\ C & 5.175631 & 4.776313 & -0.808731 \\ \text { F } & 3.494969 & 3.990292 & -2.240669 \\ C & 5.825977 & 4.733748 & 0.423565 \\ \text { F } & 6.007850 & 3.807097 & 2.593102 \\ \text { F } & 5.597901 & 5.618746 & -1.756063 \\ \text { F } & 6.865180 & 5.536094 & 0.659204\end{array}$

\section{Structure: ${ }^{5}$ INT7}

$\begin{array}{lrrr}\text { C } & 2.625186 & -1.357408 & -0.498449 \\ \mathrm{C} & 4.051867 & -1.144379 & -0.505320 \\ \mathrm{C} & 4.258188 & 0.166092 & -0.806131 \\ \mathrm{C} & 2.958877 & 0.782266 & -0.919587 \\ \mathrm{~N} & 1.987694 & -0.168145 & -0.752490 \\ \mathrm{H} & 4.796285 & -1.904192 & -0.319064 \\ \mathrm{H} & 5.200603 & 0.687056 & -0.893725 \\ \mathrm{C} & -0.596613 & 3.063819 & -0.152090 \\ \mathrm{C} & 0.018322 & 4.362400 & -0.208314 \\ \mathrm{C} & 1.304332 & 4.182106 & -0.619952 \\ \mathrm{C} & 1.515236 & 2.765967 & -0.752708 \\ \mathrm{~N} & 0.335234 & 2.105465 & -0.475363 \\ \mathrm{H} & -0.489265 & 5.295095 & -0.009836 \\ \mathrm{H} & 2.049785 & 4.941361 & -0.803817 \\ \mathrm{C} & -1.501353 & -2.418104 & -1.069704 \\ \mathrm{C} & -1.294261 & -3.839643 & -1.101063 \\ \mathrm{C} & 0.002021 & -4.067089 & -0.745157 \\ \mathrm{C} & 0.622652 & -2.783929 & -0.558721 \\ \mathrm{~N} & -0.313286 & -1.796422 & -0.759609 \\ \mathrm{H} & -2.048178 & -4.572116 & -1.347652 \\ \mathrm{H} & 0.509240 & -5.017526 & -0.664189 \\ \mathrm{C} & -2.940050 & -0.431595 & -0.930158 \\ \mathrm{C} & -4.232617 & 0.165471 & -0.713228 \\ \mathrm{C} & -4.020253 & 1.427003 & -0.253090 \\ \mathrm{C} & -2.596545 & 1.643340 & -0.247788 \\ \mathrm{~N} & -1.962220 & 0.494776 & -0.658387 \\ \mathrm{H} & -5.176977 & -0.342284 & -0.842526 \\ & & \mathrm{~S} 118 & \\ & & & \end{array}$


$\mathrm{H}$

C

C

C

C

$\mathrm{Mn}$

$\mathrm{Cl}$

$\mathrm{N}$

S

O

0

C

C

C

C

$\mathrm{H}$

C

$\mathrm{H}$

C

$\mathrm{H}$

$\mathrm{H}$

C

$\mathrm{H}$

$\mathrm{H}$

$\mathrm{H}$

C

C

C

C

C

C

F

F

F

F

F

C

C

C

C

F

C

F

C

\begin{tabular}{|c|c|c|}
\hline-4.760350 & 2.151716 & 0.049479 \\
\hline 1.994078 & -2.594647 & -0.356624 \\
\hline-1.963312 & 2.852449 & 0.043842 \\
\hline & 2.161443 & -1.009606 \\
\hline-2.738611 & -1.784571 & -1.203619 \\
\hline 0.00146 & 0.162259 & -0.708763 \\
\hline-0.0211 & 0.335553 & -2.994348 \\
\hline $0.1381 \varepsilon$ & -0.039793 & 1.21 \\
\hline-1.1 & -0.282349 & 2.27 \\
\hline-1.7 & 1.03 & 2.58 \\
\hline-1.9 & -1.38 & 1.8 \\
\hline-0.2 & -0.85 & 3.7 \\
\hline 0.1 & 0.03 & \\
\hline 0.1 & -2.19 & 3.7 \\
\hline 0.8 & -0.43 & 5.7 \\
\hline-0.1 & 1.06 & \\
\hline 0.9 & -2.63 & 4.8 \\
\hline-0.1 & -2.86 & \\
\hline 1.3 & -1.7 & \\
\hline 1.1 & 0.25 & 6 . \\
\hline 1.2 & -3.6 & \\
\hline 2.1 & $-2 \cdot 2$ & \\
\hline 1.4 & -2.68 & \\
\hline 2.8 & -3.0 & \\
\hline 2.6 & -1.4 & \\
\hline 2.8 & -3.7 & \\
\hline 2.6 & -4.4 & 1.1 \\
\hline 3.7 & -4.3 & -0.9 \\
\hline 3.4 & -5 & \\
\hline 4.5 & -5.4 & -0.7 \\
\hline 4.3 & -6.0 & 0.4 \\
\hline 5.1 & -7.1 & \\
\hline 3.3 & -6.24 & 2. \\
\hline 5.38 & -5.8 & $-1 \cdot 6$ \\
\hline 1.8 & -4.0 & \\
\hline 3.8 & -3.69 & -2.1 \\
\hline-3.937534 & -2.610415 & -1.5145 \\
\hline-4.628711 & -2.440168 & -2.71 \\
\hline-4.43 & -3.551924 & -0.6091 \\
\hline-5.768521 & -3.181864 & -3.0213 \\
\hline-4.199945 & -1.541541 & -3.6116 \\
\hline-5.57337 & -4.309180 & -0.8950 \\
\hline-3.82 & -3.747998 & 0.5 \\
\hline-6.2 & -4.12 & -2.1 \\
\hline
\end{tabular}




$\begin{array}{lrrr}\text { F } & -6.409256 & -3.002160 & -4.180523 \\ \text { F } & -6.030207 & -5.206855 & -0.015690 \\ \text { F } & -7.328727 & -4.838734 & -2.385450 \\ C & -2.771761 & 3.992797 & 0.550730 \\ C & -3.764250 & 4.610687 & -0.216861 \\ C & -2.543874 & 4.499713 & 1.836801 \\ C & -4.521132 & 5.671566 & 0.274168 \\ \text { F } & -4.011706 & 4.181066 & -1.462272 \\ C & -3.285566 & 5.564112 & 2.344774 \\ \text { F } & -1.588537 & 3.972411 & 2.606065 \\ C & -4.278539 & 6.149042 & 1.560948 \\ F & -5.466753 & 6.239417 & -0.481714 \\ \text { F } & -3.050643 & 6.028113 & 3.576252 \\ \text { F } & -4.994104 & 7.168177 & 2.040352 \\ C & 3.928894 & 3.022382 & -1.291444 \\ C & 4.549003 & 2.987385 & -2.545516 \\ C & 4.482423 & 3.867423 & -0.324139 \\ C & 5.666608 & 3.768117 & -2.833266 \\ \text { F } & 4.070001 & 2.187876 & -3.503695 \\ C & 5.595113 & 4.661212 & -0.591309 \\ \text { F } & 3.938893 & 3.929915 & 0.899137 \\ C & 6.188925 & 4.608481 & -1.851484 \\ \text { F } & 6.237275 & 3.719381 & -4.040458 \\ \text { F } & 6.101493 & 5.461692 & 0.352391 \\ \text { F } & 7.257512 & 5.361830 & -2.117016\end{array}$

\section{Structure: ${ }^{3} \mathrm{TS} 8 \mathrm{a}$}

$\begin{array}{lrrr}\text { C } & -0.257731 & 1.891601 & 2.268968 \\ \mathrm{C} & -0.090334 & 3.321948 & 2.352425 \\ \mathrm{C} & 1.084806 & 3.625139 & 1.737446 \\ \mathrm{C} & 1.664878 & 2.381388 & 1.298139 \\ \mathrm{~N} & 0.830380 & 1.343883 & 1.637717 \\ \mathrm{H} & -0.803084 & 4.006550 & 2.787543 \\ \mathrm{H} & 1.525058 & 4.600170 & 1.590014 \\ \mathrm{C} & 4.027396 & -1.107705 & 0.414013 \\ \mathrm{C} & 5.241511 & -0.405606 & 0.075887 \\ \mathrm{C} & 4.944035 & 0.919718 & 0.058654 \\ \mathrm{C} & 3.561941 & 1.048152 & 0.455449 \\ \mathrm{~N} & 3.033915 & -0.199233 & 0.668307 \\ \mathrm{H} & 6.188165 & -0.873744 & -0.148558 \\ \mathrm{H} & 5.604662 & 1.741598 & -0.174727 \\ \mathrm{C} & -1.423901 & -2.175205 & 1.502540 \\ \mathrm{C} & -2.746202 & -2.086654 & 2.066349\end{array}$




\begin{tabular}{|c|c|c|c|}
\hline C & -2.858088 & -0.853329 & 2.630014 \\
\hline $\mathrm{C}$ & -1.626245 & -0.152712 & 2.373336 \\
\hline $\mathrm{N}$ & -0.760997 & -0.988382 & 1.702764 \\
\hline $\mathrm{H}$ & -3.479561 & -2.879834 & 2.053558 \\
\hline $\mathrm{H}$ & -3.704734 & -0.445170 & 3.161602 \\
\hline C & 0.448355 & -3.474464 & 0.578295 \\
\hline C & 1.048222 & -4.716970 & 0.169119 \\
\hline $\mathrm{C}$ & 2.389820 & -4.506757 & 0.099804 \\
\hline $\mathrm{C}$ & 2.621509 & -3.120052 & 0.419098 \\
\hline $\mathrm{N}$ & 1.422632 & -2.514326 & 0.707963 \\
\hline $\mathrm{H}$ & 0.504848 & -5.632136 & -0.016544 \\
\hline $\mathrm{H}$ & 3.159307 & -5.213064 & -0.174814 \\
\hline $\mathrm{C}$ & -1.407369 & 1.197416 & 2.656814 \\
\hline $\mathrm{C}$ & 3.864269 & -2.490908 & 0.340496 \\
\hline $\mathrm{C}$ & 2.914415 & 2.262992 & 0.687831 \\
\hline $\mathrm{C}$ & -0.895124 & -3.321592 & 0.907226 \\
\hline $\mathrm{Mn}$ & 1.133300 & -0.592617 & 1.168206 \\
\hline $\mathrm{Cl}$ & 1.823072 & -1.001391 & 3.332096 \\
\hline $\mathrm{C}$ & -1.828820 & -4.456624 & 0.650467 \\
\hline $\mathrm{C}$ & -2.445037 & -4.597182 & -0.595123 \\
\hline $\mathrm{C}$ & -2.167808 & -5.377319 & 1.644793 \\
\hline $\mathrm{C}$ & -3.399806 & -5.581446 & -0.836450 \\
\hline $\mathrm{C}$ & -3.101943 & -6.387399 & 1.419455 \\
\hline $\mathrm{C}$ & -3.724136 & -6.483775 & 0.174688 \\
\hline $\mathrm{C}$ & -2.501647 & 1.977573 & 3.298597 \\
\hline $\mathrm{C}$ & -3.716547 & 2.212082 & 2.644101 \\
\hline $\mathrm{C}$ & -2.340743 & 2.540355 & 4.569339 \\
\hline $\mathrm{C}$ & -4.732244 & 2.966029 & 3.225727 \\
\hline $\mathrm{C}$ & -3.341104 & 3.303303 & 5.168363 \\
\hline $\mathrm{C}$ & -4.541679 & 3.515169 & 4.492508 \\
\hline $\mathrm{C}$ & 3.627774 & 3.513720 & 0.299060 \\
\hline $\mathrm{C}$ & 4.691836 & 4.023468 & 1.048258 \\
\hline $\mathrm{C}$ & 3.278202 & 4.201418 & -0.865154 \\
\hline $\mathrm{C}$ & 5.392212 & 5.161410 & 0.650483 \\
\hline $\mathrm{C}$ & 3.974109 & 5.327278 & -1.294706 \\
\hline $\mathrm{C}$ & 5.029348 & 5.815556 & -0.526192 \\
\hline $\mathrm{C}$ & 5.050365 & -3.339886 & 0.039627 \\
\hline $\mathrm{C}$ & 5.558014 & -4.223235 & 0.997659 \\
\hline $\mathrm{C}$ & 5.674794 & -3.314791 & -1.212100 \\
\hline $\mathrm{C}$ & 6.649134 & -5.047258 & 0.731027 \\
\hline C & 6.771535 & -4.125634 & -1.497481 \\
\hline C & 7.257149 & -4.995047 & -0.522132 \\
\hline $\mathrm{F}$ & -2.158407 & -3.739860 & -1.581304 \\
\hline $\mathrm{F}$ & -4.022333 & -5.643453 & -2.020033 \\
\hline
\end{tabular}


C

$\mathrm{H}$

$\mathrm{H}$

C

$\mathrm{H}$

$\mathrm{H}$

$\mathrm{H}$

O

O

C

C

C

C

C

C

$\mathrm{H}$

C

\begin{tabular}{|c|c|c|}
\hline-4.635490 & -7.433258 & -0.043695 \\
\hline-3.413501 & -7.254444 & 2.387608 \\
\hline-1.606787 & -5.285510 & 855813 \\
\hline 4 & -4.296406 & 2 . \\
\hline & -5.882010 & \\
\hline 8.30511 & -5.777470 & -0.7883 \\
\hline 7.35480 & -4.081380 & -2.699 \\
\hline 5.226 & -2.49 & -2.1 \\
\hline 5.072 & 3.40 & \\
\hline 6.40 & & \\
\hline & 6.9 & -0.9 \\
\hline 3.63 & 5.9 & -2.4 \\
\hline 2.24 & & -1.6 \\
\hline-3.93 & & \\
\hline-5.88 & & \\
\hline-5.50 & & \\
\hline-3.15 & .8 & 6.3 \\
\hline-1.19 & 2.3 & \\
\hline 0.62 & -0.1 & \\
\hline 0.91 & & \\
\hline 0.35 & 0.2 & -3.1 \\
\hline 1.0 & & -3 \\
\hline$-0 . \varepsilon$ & & \\
\hline 0.51 & 2.5 & -3.9 \\
\hline 1.98 & 1. & -2. \\
\hline$-1 \cdot 37$ & & \\
\hline$-1 \cdot 38$ & -0.8 & -3.7 \\
\hline-0 & 2.3 & -4. \\
\hline 1.02 & 3.4 & -4 . \\
\hline-2.32 & 1 . & \\
\hline$-1 \cdot 30$ & 3.5 & -5.3 \\
\hline-2.33 & 3.3 & -5 \\
\hline-1.3 & & \\
\hline-0.71 & 3.8 & -6.2 \\
\hline 2.3 & -1.127089 & -2.1 \\
\hline 0.04 & -2.1 & $-2 \cdot 1$ \\
\hline$-1 \cdot 315$ & 4.2 & $-1 \cdot 1$ \\
\hline-1.032139 & 2.919 & -0.9 \\
\hline-2.055 & 1.9526 & $-1 \cdot 0$ \\
\hline-3.37490 & 2.344787 & -1.516 \\
\hline-3.592129 & 3.72905 & -1.7668 \\
\hline-2.593 & 4.6597 & -1.58 \\
\hline-0.03 & & -0.6 \\
\hline-1.85 & 0.582 & -0.8 \\
\hline
\end{tabular}




\begin{tabular}{|c|c|c|c|}
\hline $\mathrm{C}$ & -4.402395 & 1.354367 & -1.659100 \\
\hline $\mathrm{H}$ & -4.568653 & 4.070361 & -2.090541 \\
\hline $\mathrm{H}$ & -2.774552 & 5.714477 & -1.766300 \\
\hline $\mathrm{C}$ & -4.119571 & 0.022240 & -1.373080 \\
\hline $\mathrm{C}$ & -2.805667 & -0.377426 & -0.996635 \\
\hline O & -0.338220 & 5.201620 & -0.877734 \\
\hline $\mathrm{C}$ & 1.027820 & 7.060177 & -1.351393 \\
\hline $\mathrm{H}$ & 1.709554 & 7.413741 & -2.123044 \\
\hline $\mathrm{H}$ & 0.372956 & 7.883261 & -1.042463 \\
\hline $\mathrm{H}$ & 1.582651 & 6.729158 & -0.470105 \\
\hline$C$ & 0.170589 & 5.948392 & -1.902604 \\
\hline O & -0.077705 & 5.758864 & -3.069053 \\
\hline C & -5.238038 & -0.983720 & -1.454653 \\
\hline$C$ & -6.188966 & -0.667994 & -2.627181 \\
\hline $\mathrm{C}$ & -4.959792 & -2.491958 & -1.569722 \\
\hline $\mathrm{H}$ & -4.589287 & -2.911896 & -0.630311 \\
\hline $\mathrm{C}$ & -6.339446 & -3.063052 & -1.968153 \\
\hline $\mathrm{H}$ & -6.913967 & -3.392716 & -1.093127 \\
\hline$C$ & -7.110831 & -1.890573 & -2.607752 \\
\hline 0 & -8.249658 & -1.931832 & -3.018416 \\
\hline C & -5.481919 & -0.684630 & -4.005255 \\
\hline $\mathrm{H}$ & -6.214615 & -0.477741 & -4.792128 \\
\hline 1 & -4.690233 & 0.069595 & -4.052002 \\
\hline $\mathrm{H}$ & -5.023795 & -1.655540 & -4.222376 \\
\hline $\mathrm{H}$ & -6.288696 & -3.918637 & -2.648866 \\
\hline ! & -4.208152 & -2.700554 & -2.334770 \\
\hline 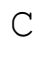 & -6.841959 & 0.682623 & -2.346599 \\
\hline$C$ & -5.767923 & 1.790283 & -2.189697 \\
\hline $\mathrm{H}$ & -7.429777 & 0.599835 & -1.424197 \\
\hline $\mathrm{H}$ & -7.546321 & 0.953880 & -3.140194 \\
\hline & -6.174207 & 2.583365 & -1.549137 \\
\hline & -5.599478 & 2.263102 & -3.167149 \\
\hline & -2.569099 & -1.425430 & -0.859589 \\
\hline & -0.507609 & 0.198470 & -0.603892 \\
\hline & -5.838049 & -0.858267 & -0.536231 \\
\hline
\end{tabular}

\section{Structure: ${ }^{5}$ TS8a}

$\begin{array}{lrrr}\mathrm{C} & 0.334205 & -1.842009 & 2.266827 \\ \mathrm{C} & 0.215253 & -3.277256 & 2.355775 \\ \mathrm{C} & -0.955587 & -3.621919 & 1.754641 \\ \mathrm{C} & -1.580411 & -2.399139 & 1.318574 \\ \mathrm{~N} & -0.779690 & -1.333227 & 1.646159 \\ \mathrm{H} & 0.956713 & -3.935953 & 2.782783\end{array}$




\begin{tabular}{|c|c|c|c|}
\hline $\mathrm{H}$ & -1.362190 & -4.611742 & 1.609216 \\
\hline $\mathrm{C}$ & -4.049257 & 1.020505 & 0.437515 \\
\hline C & -5.241736 & 0.279411 & 0.116298 \\
\hline C & -4.904164 & -1.037408 & 0.099605 \\
\hline C & -3.517834 & -1.125458 & 0.482552 \\
\hline $\mathrm{N}$ & -3.022269 & 0.141430 & 0.684486 \\
\hline $\mathrm{H}$ & -6.205318 & 0.716607 & -0.098163 \\
\hline $\mathrm{H}$ & -5.542626 & -1.879163 & -0.124515 \\
\hline $\mathrm{C}$ & 1.381839 & 2.238863 & 1.438600 \\
\hline $\mathrm{C}$ & 2.713626 & 2.197858 & 1.988212 \\
\hline C & 2.866067 & 0.976946 & 2.569283 \\
\hline $\mathrm{C}$ & 1.649040 & 0.239915 & 2.337762 \\
\hline $\mathrm{N}$ & 0.755998 & 1.040116 & 1.666048 \\
\hline $\mathrm{H}$ & 3.422695 & 3.012333 & 1.955341 \\
\hline $\mathrm{H}$ & 3.729579 & 0.600059 & 3.096920 \\
\hline $\mathrm{C}$ & -0.541757 & 3.480629 & 0.537764 \\
\hline $\mathrm{C}$ & -1.178196 & 4.702146 & 0.119698 \\
\hline $\mathrm{C}$ & -2.515483 & 4.460857 & 0.078955 \\
\hline C & -2.708648 & 3.073649 & 0.422515 \\
\hline $\mathrm{N}$ & -1.494196 & 2.500937 & 0.703425 \\
\hline $\mathrm{H}$ & -0.658794 & 5.625176 & -0.093448 \\
\hline $\mathrm{H}$ & -3.306019 & 5.144104 & -0.194311 \\
\hline $\mathrm{C}$ & 1.466837 & -1.112407 & 2.639449 \\
\hline $\mathrm{C}$ & -3.933248 & 2.407412 & 0.360634 \\
\hline $\mathrm{C}$ & -2.837583 & -2.320446 & 0.717173 \\
\hline $\mathrm{C}$ & 0.810744 & 3.363558 & 0.837328 \\
\hline $\mathrm{Mn}$ & -1.155921 & 0.592927 & 1.178455 \\
\hline $\mathrm{Cl}$ & -1.757178 & 1.005145 & 3.359217 \\
\hline $\mathrm{C}$ & 1.714061 & 4.515058 & 0.546147 \\
\hline $\mathrm{C}$ & 2.363461 & 4.610818 & -0.686799 \\
\hline $\mathrm{C}$ & 1.996497 & 5.494293 & 1.501339 \\
\hline C & 3.298542 & 5.608463 & -0.949645 \\
\hline $\mathrm{C}$ & 2.908811 & 6.518583 & 1.251930 \\
\hline C & 3.566902 & 6.569479 & 0.023051 \\
\hline $\mathrm{C}$ & 2.583220 & -1.855500 & 3.287289 \\
\hline $\mathrm{C}$ & 3.801695 & -2.068312 & 2.632442 \\
\hline $\mathrm{C}$ & 2.439800 & -2.404868 & 4.566087 \\
\hline $\mathrm{C}$ & 4.837793 & -2.788231 & 3.221131 \\
\hline $\mathrm{C}$ & 3.460987 & -3.133512 & 5.172574 \\
\hline $\mathrm{C}$ & 4.664782 & -3.324208 & 4.496025 \\
\hline $\mathrm{C}$ & -3.523089 & -3.591300 & 0.343569 \\
\hline C & -4.550911 & -4.134201 & 1.119478 \\
\hline C & -3.187594 & -4.262416 & -0.834165 \\
\hline C & -5.229221 & -5.290089 & 0.735421 \\
\hline
\end{tabular}




\begin{tabular}{|c|c|c|c|}
\hline C & -3.863479 & -5.405238 & -1.250664 \\
\hline C & -4.881560 & -5.927618 & -0.454973 \\
\hline C & -5.146972 & 3.218405 & 0.066817 \\
\hline C & -5.667268 & 4.096196 & 1.023235 \\
\hline C & -5.784937 & 3.165626 & -1.177332 \\
\hline C & -6.783451 & 4.887923 & 0.762784 \\
\hline C & -6.906655 & 3.943905 & -1.456328 \\
\hline C & -7.404354 & 4.808345 & -0.482621 \\
\hline $\mathrm{F}$ & 2.127268 & 3.701328 & -1.638491 \\
\hline $\mathrm{F}$ & 3.953284 & 5.630205 & -2.117199 \\
\hline F & 4.457800 & 7.533008 & -0.216998 \\
\hline F & 3.166452 & 7.441933 & 2.183187 \\
\hline $\mathrm{F}$ & 1.400387 & 5.448092 & 2.698159 \\
\hline $\mathrm{F}$ & -5.083940 & 4.194921 & 2.223379 \\
\hline $\mathrm{F}$ & -7.262047 & 5.717521 & 1.695448 \\
\hline $\mathrm{F}$ & -8.476337 & 5.559493 & -0.742819 \\
\hline F & -7.502348 & 3.873462 & -2.651217 \\
\hline $\mathrm{F}$ & -5.326116 & 2.350045 & -2.131025 \\
\hline $\mathrm{F}$ & -4.918937 & -3.527921 & 2.253026 \\
\hline $\mathrm{F}$ & -6.208201 & -5.788205 & 1.495387 \\
\hline $\mathrm{F}$ & -5.525529 & -7.031785 & -0.835634 \\
\hline $\mathrm{F}$ & -3.535979 & -6.005934 & -2.401202 \\
\hline $\mathrm{F}$ & -2.192908 & -3.799630 & -1.610412 \\
\hline $\mathrm{F}$ & 4.003044 & -1.570371 & 1.405054 \\
\hline $\mathrm{F}$ & 5.990461 & -2.973562 & 2.567990 \\
\hline $\mathrm{F}$ & 5.650122 & -4.018868 & 5.067626 \\
\hline $\mathrm{F}$ & 3.295441 & -3.646781 & 6.395354 \\
\hline $\mathrm{F}$ & 1.292543 & -2.244931 & 5.234729 \\
\hline $\mathrm{N}$ & -0.602468 & 0.148718 & -0.607184 \\
\hline S & -0.958053 & 0.964764 & -2.028114 \\
\hline $\mathrm{C}$ & -0.397497 & -0.274900 & -3.189738 \\
\hline C & -1.084793 & -1.490449 & -3.251307 \\
\hline $\mathrm{C}$ & 0.796919 & -0.083025 & -3.881200 \\
\hline C & -0.545122 & -2.536087 & -3.991298 \\
\hline $\mathrm{H}$ & -2.001410 & -1.620903 & -2.688223 \\
\hline C & 1.319552 & -1.140190 & -4.626065 \\
\hline $\mathrm{H}$ & 1.318690 & 0.863770 & -3.800102 \\
\hline C & 0.673609 & -2.382455 & -4.675017 \\
\hline $\mathrm{H}$ & -1.042166 & -3.501401 & -4.000004 \\
\hline $\mathrm{H}$ & 2.260791 & -1.005777 & -5.153686 \\
\hline C & 1.274201 & -3.552567 & -5.412871 \\
\hline $\mathrm{H}$ & 2.281963 & -3.325780 & -5.775283 \\
\hline $\mathrm{H}$ & 1.321640 & -4.426218 & -4.755147 \\
\hline $\mathrm{H}$ & 0.659591 & -3.831258 & -6.278465 \\
\hline
\end{tabular}




\begin{tabular}{|c|c|c|c|}
\hline 0 & -2.422190 & 1.100913 & -2.098543 \\
\hline O & -0.121425 & 2.165404 & -2.204227 \\
\hline $\mathrm{C}$ & 1.355536 & -4.252622 & -1.184457 \\
\hline $\mathrm{C}$ & 1.053249 & -2.933073 & -0.974419 \\
\hline C & 2.067806 & -1.954828 & -1.118429 \\
\hline C & 3.398783 & -2.334885 & -1.513728 \\
\hline $\mathrm{C}$ & 3.635719 & -3.717617 & -1.754657 \\
\hline C & 2.644490 & -4.658490 & -1.588222 \\
\hline $\mathrm{H}$ & 0.049718 & -2.634762 & -0.704863 \\
\hline $\mathrm{C}$ & 1.842343 & -0.586384 & -0.893379 \\
\hline $\mathrm{C}$ & 4.420347 & -1.335076 & -1.630547 \\
\hline $\mathrm{H}$ & 4.621516 & -4.049812 & -2.059308 \\
\hline $\mathrm{H}$ & 2.840546 & -5.712266 & -1.756443 \\
\hline $\mathrm{C}$ & 4.116078 & -0.005950 & -1.353553 \\
\hline $\mathrm{C}$ & 2.788340 & 0.379824 & -1.017022 \\
\hline O & 0.389581 & -5.226179 & -0.901414 \\
\hline $\mathrm{C}$ & -0.925704 & -7.120705 & -1.378189 \\
\hline $\mathrm{H}$ & -1.606482 & -7.484814 & -2.145804 \\
\hline $\mathrm{H}$ & -0.245940 & -7.929356 & -1.085225 \\
\hline $\mathrm{H}$ & -1.478810 & -6.813222 & -0.487405 \\
\hline C & -0.102661 & -5.982292 & -1.927735 \\
\hline O & 0.135812 & -5.781099 & -3.094164 \\
\hline $\mathrm{C}$ & 5.229250 & 1.008653 & -1.387961 \\
\hline $\mathrm{C}$ & 6.232329 & 0.703180 & -2.518543 \\
\hline $\mathrm{C}$ & 4.945340 & 2.515226 & -1.511215 \\
\hline $\mathrm{H}$ & 4.528886 & 2.928885 & -0.588190 \\
\hline C & 6.337301 & 3.096869 & -1.846013 \\
\hline $\mathrm{H}$ & 6.870617 & 3.427057 & -0.945455 \\
\hline $\mathrm{C}$ & 7.144463 & 1.931409 & -2.454541 \\
\hline O & 8.300301 & 1.981761 & -2.813467 \\
\hline C & 5.585923 & 0.720396 & -3.925965 \\
\hline H & 6.352366 & 0.514919 & -4.680440 \\
\hline $\mathrm{H}$ & 4.797973 & -0.034647 & -4.007222 \\
\hline $\mathrm{H}$ & 5.136479 & 1.690878 & -4.162273 \\
\hline $\mathrm{H}$ & 6.310667 & 3.954335 & -2.525807 \\
\hline $\mathrm{H}$ & 4.227769 & 2.720957 & -2.308922 \\
\hline C & 6.880488 & -0.644851 & -2.215223 \\
\hline C & 5.807160 & -1.759310 & -2.112295 \\
\hline $\mathrm{H}$ & 7.424911 & -0.563495 & -1.266409 \\
\hline $\mathrm{H}$ & 7.621817 & -0.907729 & -2.977371 \\
\hline $\mathrm{H}$ & 6.192400 & -2.555648 & -1.462915 \\
\hline $\mathrm{H}$ & 5.680153 & -2.224271 & -3.099797 \\
\hline $\mathrm{H}$ & 2.538638 & 1.425691 & -0.887481 \\
\hline & 0.569229 & -0.201708 & -0.644788 \\
\hline
\end{tabular}




\section{Structure: ${ }^{1}$ TS8b}

\author{
C
}

C

C

C

$\mathrm{N}$

$\mathrm{H}$

$\mathrm{H}$

C

C

C

C

$\mathrm{N}$

$\mathrm{H}$

$\mathrm{H}$

C

C

C

C

$\mathrm{N}$

$\mathrm{H}$

$\mathrm{H}$

C

C

C

C

$\mathrm{N}$

$\mathrm{H}$

$\mathrm{H}$

C

C

C

C

$\mathrm{Mn}$

$\mathrm{Cl}$

C

C

C

C

C

C
$1.482111-2.009813-2.151888$

$2.666821-2.830048-2.249566$

$3.670079-2.145159-1.639138$

$3.105552-0.889629-1.199692$

$1.787188-0.821126-1.519039$

$\begin{array}{llll}2.720634 & -3.801712 & -2.718264\end{array}$

$\begin{array}{llll}4.692867 & -2.458456 & -1.495577\end{array}$

$2.166995 \quad 3.232163-0.515738$

$\begin{array}{lll}3.448460 & 3.632817 & 0.029348\end{array}$

$\begin{array}{lll}4.185571 & 2.502209 & 0.174229\end{array}$

$\begin{array}{llll}3.376064 & 1.413863 & -0.323402\end{array}$

$\begin{array}{lll}2.135899 & 1.889571 & -0.705087\end{array}$

$\begin{array}{lll}3.728568 & 4.642688 & 0.289664\end{array}$

$\begin{array}{lll}5.192851 & 2.403223 & 0.551783\end{array}$

$\begin{array}{lll}-2.318431 & 0.059413 & -2.016120\end{array}$

$\begin{array}{lll}-3.134175 & -1.035471 & -2.488126\end{array}$

$\begin{array}{llll}-2.288359 & -2.062413 & -2.772528\end{array}$

$\begin{array}{lll}-0.957717 & -1.607387 & -2.451015\end{array}$

$-0.998353-0.325585-1.986918$

$\begin{array}{lll}-4.207315 & -1.029227 & -2.597953\end{array}$

$\begin{array}{lll}-2.546839 & -3.042139 & -3.146122\end{array}$

$\begin{array}{lll}-1.969279 & 2.446108 & -1.383060\end{array}$

$\begin{array}{llll}-2.449539 & 3.810446 & -1.314677\end{array}$

$\begin{array}{lll}-1.356317 & 4.599994 & -1.155293\end{array}$

$\begin{array}{llll}-0.212226 & 3.717467 & -1.064682\end{array}$

$\begin{array}{lll}-0.617482 & 2.416272 & -1.231688\end{array}$

$\begin{array}{lll}-3.481091 & 4.115632 & -1.417880\end{array}$

$\begin{array}{llll}-1.324330 & 5.677365 & -1.093420\end{array}$

$\begin{array}{llll}0.207163 & -2.398779 & -2.557153\end{array}$

$\begin{array}{llll}1.089091 & 4.117231 & -0.753994\end{array}$

$\begin{array}{llll}3.833729 & 0.120244 & -0.526517\end{array}$

$\begin{array}{lll}-2.788029 & 1.334192 & -1.663475\end{array}$

$\begin{array}{llll}0.556789 & 0.779040 & -1.284444\end{array}$

$\begin{array}{lll}1.060567 & 1.326981 & -3.500708\end{array}$

$\begin{array}{lll}-4.266263 & 1.519465 & -1.573064\end{array}$

$\begin{array}{lll}-4.864992 & 1.739349 & -0.326342\end{array}$

$\begin{array}{lll}-5.121874 & 1.381733 & -2.671593\end{array}$

$\begin{array}{lll}-6.245379 & 1.748471 & -0.162017\end{array}$

$\begin{array}{lll}-6.509270 & 1.397047 & -2.532415\end{array}$

$\begin{array}{lll}-7.070885 & 1.564381 & -1.267294\end{array}$ 


\begin{tabular}{|c|c|c|c|}
\hline & 0.044731 & -3.787597 & -3.071765 \\
\hline C & 0.225757 & -4.893471 & -2.234047 \\
\hline & -0.303105 & -4.037108 & -4.402870 \\
\hline & 0.073189 & -6.197680 & -2.695648 \\
\hline & -0.462818 & -5.333849 & -4.886807 \\
\hline & -0.271918 & -6.416178 & -4.028915 \\
\hline & 5.189577 & -0.250251 & -0.025633 \\
\hline & 6.284529 & -0.374502 & -0.884012 \\
\hline & 5.381530 & -0.559965 & 1.323144 \\
\hline & 7.526825 & -0.801697 & -0.424205 \\
\hline & 6.611474 & -1.001378 & 1.803282 \\
\hline & 7.686451 & -1.117756 & 0.925414 \\
\hline & 1.357807 & 5.568066 & -0.564249 \\
\hline & 2.244498 & 6.243846 & -1.410649 \\
\hline & 0.774674 & 6.298019 & 0.478732 \\
\hline & 2.536375 & 7.594368 & -1.240701 \\
\hline & 1.052471 & 7.651071 & 0.663961 \\
\hline & 1.935509 & 8.298734 & -0.198319 \\
\hline & -4.110672 & 1.898532 & 0.766412 \\
\hline & -6.780138 & 1.893950 & 1.056315 \\
\hline & -8.396057 & 1.533894 & -1.105379 \\
\hline & -7.299051 & 1.237985 & -3.597462 \\
\hline $\mathrm{F}$ & -4.613441 & 1.189987 & -3.893949 \\
\hline $\mathrm{F}$ & 2.838597 & 5.585428 & -2.412199 \\
\hline $\mathrm{F}$ & 3.382339 & 8.217349 & -2.066538 \\
\hline $\mathrm{F}$ & 2.206227 & 9.593552 & -0.026565 \\
\hline $\mathrm{F}$ & 0.483549 & 8.328961 & 1.665471 \\
\hline $\mathrm{F}$ & -0.068680 & 5.704914 & 1.326272 \\
\hline $\mathrm{F}$ & 6.139023 & -0.101814 & -2.188610 \\
\hline $\mathrm{F}$ & 8.562661 & -0.913049 & -1.263421 \\
\hline $\mathrm{E}$ & 8.873147 & -1.533027 & 1.373727 \\
\hline $\mathrm{F}$ & 6.763430 & -1.310305 & 3.091982 \\
\hline $\mathrm{F}$ & 4.367861 & -0.443182 & 2.181799 \\
\hline $\mathrm{F}$ & 0.549946 & -4.714250 & -0.942866 \\
\hline $\mathrm{E}$ & 0.249480 & -7.235092 & -1.871958 \\
\hline $\mathrm{F}$ & -0.421572 & -7.660957 & -4.482713 \\
\hline $\mathrm{F}$ & -0.793119 & -5.546359 & -6.163090 \\
\hline $\mathrm{E}$ & -0.493716 & -3.014943 & -5.242622 \\
\hline $\mathrm{N}$ & 0.023898 & 0.365332 & 0.531409 \\
\hline $\mathrm{s}$ & 0.502413 & 1.228398 & 1.862896 \\
\hline c & -0.699306 & 0.737023 & 3.093965 \\
\hline c & -0.301314 & 0.376227 & 4.381154 \\
\hline c & -2.048207 & 0.802573 & 2.754116 \\
\hline c & -1.274500 & -0.004519 & 5.304314 \\
\hline
\end{tabular}




\begin{tabular}{|c|c|c|c|}
\hline $\mathrm{H}$ & 0.750918 & 0.387469 & 4.639879 \\
\hline C & -3.011062 & 0.436252 & 3.689003 \\
\hline $\mathrm{H}$ & -2.329100 & 1.103458 & 1.755946 \\
\hline C & -2.636490 & -0.003933 & 4.965362 \\
\hline $\mathrm{H}$ & -0.971302 & -0.316775 & 6.301178 \\
\hline $\mathrm{H}$ & -4.060375 & 0.453392 & 3.416558 \\
\hline C & -3.674900 & -0.499846 & 5.942576 \\
\hline $\mathrm{H}$ & -3.534003 & -1.572415 & 6.132504 \\
\hline $\mathrm{H}$ & -3.596121 & 0.012114 & 6.909463 \\
\hline $\mathrm{H}$ & -4.686345 & -0.359991 & 5.552135 \\
\hline O & 1.842798 & 0.834955 & 2.350060 \\
\hline O & 0.262764 & 2.661546 & 1.593681 \\
\hline $\mathrm{H}$ & 0.095719 & -0.934121 & 0.909398 \\
\hline C & -4.851317 & -1.876109 & 1.803224 \\
\hline C & -4.399477 & -2.646122 & 2.847137 \\
\hline C & -3.021444 & -2.983031 & 2.928422 \\
\hline C & -2.104664 & -2.479782 & 1.947100 \\
\hline $\mathrm{C}$ & -2.626495 & -1.683181 & 0.897295 \\
\hline C & -3.971195 & -1.403870 & 0.813483 \\
\hline $\mathrm{H}$ & -5.089472 & -3.003167 & 3.604310 \\
\hline $\mathrm{C}$ & -2.532156 & -3.832334 & 3.954897 \\
\hline $\mathrm{C}$ & -0.706903 & -2.807265 & 2.037701 \\
\hline $\mathrm{H}$ & -1.958051 & -1.270024 & 0.157559 \\
\hline $\mathrm{H}$ & -4.372509 & -0.821834 & -0.005659 \\
\hline C & -0.293280 & -3.729783 & 3.013596 \\
\hline $\mathrm{C}$ & -1.208726 & -4.216002 & 3.971506 \\
\hline O & -6.207533 & -1.576959 & 1.622533 \\
\hline C & -8.298188 & -0.618288 & 2.129701 \\
\hline $\mathrm{H}$ & -8.834047 & -0.084923 & 2.915313 \\
\hline $\mathrm{H}$ & -8.339062 & -0.038153 & 1.203097 \\
\hline $\mathrm{H}$ & -8.767590 & -1.588037 & 1.938390 \\
\hline C & -6.862367 & -0.810796 & 2.550361 \\
\hline O & -6.347233 & -0.364250 & 3.546790 \\
\hline $\mathrm{C}$ & 1.125454 & -4.230505 & 2.921016 \\
\hline $\mathrm{C}$ & 2.092693 & -3.055668 & 2.676883 \\
\hline $\mathrm{C}$ & 1.784469 & -5.046289 & 4.049201 \\
\hline $\mathrm{H}$ & 1.408070 & -6.072566 & 4.101881 \\
\hline $\mathrm{C}$ & 3.287346 & -4.983490 & 3.675828 \\
\hline $\mathrm{H}$ & 3.601476 & -5.861109 & 3.097446 \\
\hline C & 3.446834 & -3.749511 & 2.762968 \\
\hline O & 4.471591 & -3.415740 & 2.203504 \\
\hline $\mathrm{C}$ & 2.051964 & -2.041911 & 3.844590 \\
\hline $\mathrm{H}$ & 2.658104 & -1.168901 & 3.607050 \\
\hline $\mathrm{H}$ & 1.025393 & -1.713897 & 4.009681 \\
\hline
\end{tabular}


H

$\mathrm{H}$

$\mathrm{H}$

C

C

$\mathrm{H}$

$\mathrm{H}$

$\mathrm{H}$

$\mathrm{H}$

$\mathrm{H}$

$\mathrm{H}$

$\begin{array}{rrr}2.418517 & -2.477803 & 4.781062 \\ 3.964198 & -4.915399 & 4.533801 \\ 1.601834 & -4.581736 & 5.024013 \\ 1.773076 & -2.421445 & 1.326382 \\ 0.269178 & -2.238674 & 1.078058 \\ 2.182029 & -3.058719 & 0.534099 \\ 2.278246 & -1.457933 & 1.249268 \\ -0.006762 & -2.453256 & 0.044770 \\ -0.858191 & -4.912621 & 4.727904 \\ -3.223799 & -4.203610 & 4.707038 \\ 1.170527 & -4.859454 & 2.013687\end{array}$

Structure: ${ }^{3} \mathrm{TS} 8 \mathrm{~b}$

C

C

C

C

N

$\mathrm{H}$

$\mathrm{H}$

C

C

C

C

N

$\mathrm{H}$

$\mathrm{H}$

C

C

C

C

N

$\mathrm{H}$

$\mathrm{H}$

C

C

C

C

N

$\mathrm{H}$

$\mathrm{H}$

C

C
$-1.528331-1.916260$

$-2.722253-2.719943$

$-3.699902-2.060692$

$-3.127743-0.823705$

$-1.805310-0.758686$

$\begin{array}{ll}-2.793707 & -3.672879\end{array}$

$\begin{array}{lll}-4.719191 & -2.373245\end{array}$

$-2.1371113 .283823$

$-3.425972$

$-4.168510$

$-3.362561$

$-2.122095$

$-3.706703$

$-5.182057$

2.293674

3.078837

2.215505

0.892330

0.966680

4.152227

2.453677

2.000307

2.492546

1.407533

0.242190

0.633530

3.532867

1. 390768

$-0.268678$

$-1.060321$
3.692181

2.566017

1.460601

1.919335

4.710976

2.479345

0.074449

$-1.007077$

$-2.000658$

$-1.551441$

$-0.291740$

$-1.009424$

$-2.960908$

2. 409712

3.760842

4.567653

3. 714838

2.412955

4.048526

5.642925

$-2.316810$

4.149227
2.156486

2. 242120

1.564039

1.098598

1.466992

2.745823

1.393386

0.461482

$-0.041563$

$-0.210955$

0.238134

0.612860

$-0.264152$

$-0.574788$

1. 925619

2. 466705

2.809832

2.464473

1.919074

2. 577734

3. 243049

1.187577

1.053273

0.922176

0.912192

1.085054

1.103726

0.826124

2. 605619

0.661767 


\begin{tabular}{|c|c|c|c|}
\hline $\mathrm{C}$ & -3.840820 & 0.166048 & 0.421725 \\
\hline $\mathrm{C}$ & 2.801938 & 1.314956 & 1.520196 \\
\hline Mn & -0.568798 & 0.805033 & 1.196343 \\
\hline $\mathrm{Cl}$ & -1.027832 & 1.441126 & 3.391268 \\
\hline $\mathrm{C}$ & 4.283914 & 1.485777 & 1.485315 \\
\hline C & 4.958018 & 1.636689 & 0.267940 \\
\hline $\mathrm{C}$ & 5.074908 & 1.430497 & 2.638993 \\
\hline $\mathrm{C}$ & 6.345583 & 1.672552 & 0.188515 \\
\hline $\mathrm{C}$ & 6.468115 & 1.462172 & 2.584793 \\
\hline $\mathrm{C}$ & 7.105457 & 1.569981 & 1.349722 \\
\hline C & -0.136523 & -3.687935 & 3.172265 \\
\hline C & -0.294501 & -4.819453 & 2.364796 \\
\hline C & 0.159911 & -3.899937 & 4.521958 \\
\hline $\mathrm{C}$ & -0.173335 & -6.110251 & 2.870600 \\
\hline $\mathrm{C}$ & 0.292931 & -5.182719 & 5.050729 \\
\hline $\mathrm{C}$ & 0.122418 & -6.289951 & 4.221347 \\
\hline C & -5.208958 & -0.181799 & -0.061245 \\
\hline $\mathrm{C}$ & -6.322876 & -0.130660 & 0.779934 \\
\hline C & -5.402333 & -0.638858 & -1.366836 \\
\hline $\mathrm{C}$ & -7.584085 & -0.531511 & 0.345821 \\
\hline C & -6.650896 & -1.054558 & -1.820843 \\
\hline $\mathrm{C}$ & -7.744252 & -0.996688 & -0.959354 \\
\hline $\mathrm{C}$ & -1.306469 & 5.606936 & 0.483035 \\
\hline $\mathrm{C}$ & -2.087129 & 6.318013 & 1.400507 \\
\hline $\mathrm{C}$ & -0.801528 & 6.309866 & -0.616600 \\
\hline $\mathrm{C}$ & -2.354461 & 7.675744 & 1.240632 \\
\hline C & -1.050832 & 7.669735 & -0.791437 \\
\hline $\mathrm{C}$ & -1.831606 & 8.352126 & 0.139953 \\
\hline $\mathrm{F}$ & 4.274422 & 1.707613 & -0.881628 \\
\hline $\mathrm{F}$ & 6.951660 & 1.763605 & -1.002779 \\
\hline $\mathrm{F}$ & 8.438770 & 1.561812 & 1.270929 \\
\hline $\mathrm{F}$ & 7.192231 & 1.379377 & 3.704397 \\
\hline $\mathrm{F}$ & 4.496270 & 1.304358 & 3.838502 \\
\hline $\mathrm{F}$ & -2.605766 & 5.689996 & 2.461900 \\
\hline $\mathrm{F}$ & -3.103311 & 8.332028 & 2.132986 \\
\hline $\mathrm{F}$ & -2.078008 & 9.654111 & -0.021855 \\
\hline $\mathrm{F}$ & -0.553533 & 8.321598 & -1.847956 \\
\hline $\mathrm{F}$ & -0.052714 & 5.681460 & -1.527516 \\
\hline $\mathrm{F}$ & -6.183540 & 0.291584 & 2.043963 \\
\hline $\mathrm{F}$ & -8.637622 & -0.477148 & 1.168969 \\
\hline$F$ & -8.949257 & -1.386070 & -1.382725 \\
\hline $\mathrm{F}$ & -6.805658 & -1.500191 & -3.069623 \\
\hline F & -4.370615 & -0.686879 & -2.212024 \\
\hline$F$ & -0.569790 & -4.678039 & 1.055719 \\
\hline
\end{tabular}




\begin{tabular}{|c|c|c|c|}
\hline$F$ & -0.330187 & -7.171915 & 2.072904 \\
\hline $\mathrm{F}$ & 0.244617 & -7.521718 & 4.718937 \\
\hline $\mathrm{F}$ & 0.577251 & -5.358401 & 6.344166 \\
\hline $\mathrm{F}$ & 0.331234 & -2.854861 & 5.337684 \\
\hline $\mathrm{N}$ & -0.051958 & 0.353914 & -0.561192 \\
\hline S & -0.534213 & 1.154278 & -1.953172 \\
\hline $\mathrm{C}$ & 0.726674 & 0.637696 & -3.109258 \\
\hline C & 0.373665 & 0.150388 & -4.367364 \\
\hline $\mathrm{C}$ & 2.062318 & 0.796715 & -2.747670 \\
\hline $\mathrm{C}$ & 1.383798 & -0.252533 & -5.238632 \\
\hline $\mathrm{H}$ & -0.671432 & 0.087816 & -4.645116 \\
\hline C & 3.061558 & 0.404887 & -3.633973 \\
\hline $\mathrm{H}$ & 2.308795 & 1.191496 & -1.772256 \\
\hline $\mathrm{C}$ & 2.736488 & -0.150902 & -4.878944 \\
\hline $\mathrm{H}$ & 1.117925 & -0.659538 & -6.211584 \\
\hline $\mathrm{H}$ & 4.102239 & 0.493966 & -3.342895 \\
\hline $\mathrm{C}$ & 3.816741 & -0.642774 & -5.811893 \\
\hline $\mathrm{H}$ & 3.631443 & -1.686289 & -6.097261 \\
\hline $\mathrm{H}$ & 3.839988 & -0.054920 & -6.738400 \\
\hline $\mathrm{H}$ & 4.801306 & -0.585977 & -5.341839 \\
\hline O & -1.837584 & 0.657010 & -2.436277 \\
\hline O & -0.371528 & 2.602721 & -1.744794 \\
\hline $\mathrm{H}$ & -0.105985 & -0.990790 & -0.908807 \\
\hline $\mathrm{C}$ & 4.870975 & -1.968295 & -1.761057 \\
\hline $\mathrm{C}$ & 4.415689 & -2.751243 & -2.794336 \\
\hline $\mathrm{C}$ & 3.034711 & -3.072943 & -2.876274 \\
\hline $\mathrm{C}$ & 2.117815 & -2.536066 & -1.913751 \\
\hline $\mathrm{C}$ & 2.639753 & -1.715098 & -0.885579 \\
\hline $\mathrm{C}$ & 3.988622 & -1.457983 & -0.793759 \\
\hline $\mathrm{H}$ & 5.107784 & -3.140662 & -3.533691 \\
\hline $\mathrm{C}$ & 2.544502 & -3.953873 & -3.875183 \\
\hline C & 0.719946 & -2.869349 & -1.990687 \\
\hline $\mathrm{H}$ & 1.971668 & -1.278019 & -0.159819 \\
\hline $\mathrm{H}$ & 4.390138 & -0.867180 & 0.017952 \\
\hline $\mathrm{C}$ & 0.309541 & -3.836022 & -2.929032 \\
\hline $\mathrm{C}$ & 1.223272 & -4.347476 & -3.873443 \\
\hline O & 6.232232 & -1.705636 & -1.570157 \\
\hline $\mathrm{C}$ & 8.341847 & -0.772459 & -2.049386 \\
\hline $\mathrm{H}$ & 8.883871 & -0.215664 & -2.814150 \\
\hline $\mathrm{H}$ & 8.401929 & -0.235306 & -1.098358 \\
\hline $\mathrm{H}$ & 8.789750 & -1.760418 & -1.906074 \\
\hline $\mathrm{C}$ & 6.899365 & -0.912185 & -2.466317 \\
\hline O & 6.386348 & -0.409384 & -3.436402 \\
\hline $\mathrm{C}$ & -1.095733 & -4.361886 & -2.797728 \\
\hline
\end{tabular}


C
C
H
C
H
C
O
C
H
H
H
H
H
C
C
H
H
H
H
H
H

O

$\mathrm{H}$

$\mathrm{H}$

$\begin{array}{rrr}-2.083086 & -3.196579 & -2.596330 \\ -1.750701 & -5.244215 & -3.876565 \\ -1.351904 & -6.263259 & -3.886297 \\ -3.249077 & -5.196803 & -3.482410 \\ -3.536898 & -6.056332 & -2.864950 \\ -3.421245 & -3.930146 & -2.616891 \\ -4.437372 & -3.605969 & -2.038483 \\ -2.085039 & -2.250200 & -3.820717 \\ -2.675770 & -1.360348 & -3.612348 \\ -1.064520 & -1.941257 & -4.048583 \\ -2.492859 & -2.737422 & -4.713666 \\ -3.938654 & -5.176921 & -4.332639 \\ -1.592401 & -4.822152 & -4.874698 \\ -1.757619 & -2.492984 & -1.282459 \\ -0.256052 & -2.285149 & -1.044609 \\ -2.152294 & -3.095048 & -0.457075 \\ -2.273350 & -1.534011 & -1.245498 \\ 0.027122 & -2.462861 & -0.004871 \\ 0.875710 & -5.075506 & -4.600821 \\ 3.235589 & -4.348736 & -4.615703 \\ -1.114448 & -4.946990 & -1.860729\end{array}$

\section{Structure: ${ }^{5} \mathrm{TS} 8 \mathrm{~b}$}

C

C

C

C

$\mathrm{N}$

$\mathrm{H}$

$\mathrm{H}$

C

C

C

C

$\mathrm{N}$

$\mathrm{H}$

$\mathrm{H}$

C

C

C

C

$\mathrm{N}$

$\mathrm{H}$
$-1.510042$

$-2.691062$

$-3.670253$

$-3.110132$

$-1.795247$

$-2.755203$

$-4.682693$

$-2.178944$

$-3.472038$

$-4.198934$

$-3.380450$

$-2.147779$

$-3.763669$

$-5.208643$

2. 290110

3.083432

2. 226310

0.900450

0.967002

4.156811
$-1.927160$

$-2.751399$

$-2.102255$

$-0.853707$

$-0.771689$

$-3.708497$

$-2.428537$

3. 279815

3. 673211

2. 537883

1. 441487

1. 919075

4.688427

2.438200

0.107251

$-0.962887$

$-1.958526$

$-1.522598$

$-0.264878$

$-0.957026$
2.144561

2. 207510

1. 520167

1.072589

1.458152

2.704214

1. 334676

0.476528

$-0.028887$

$-0.202042$

0.247629

0.633667

$-0.253929$

$-0.573069$

1.936364

2.488698

2.840599

2.486900

1.934135

2.599408 


$$
\begin{array}{r}
2.470749 \\
1.973743 \\
2.452973 \\
1.361278 \\
0.200959 \\
0.601445 \\
3.491489 \\
1.338469 \\
-0.250685
\end{array}
$$

$-1.104605$

$-3.836235$

2. 787461

$-0.583064$

$-1.074441$

4. 267307

4.932509

5.066306

6.319013

6.458803

7.087143

$-0.107420$

$-0.238897$

0.173705

$-0.107116$

0.316836

0.172671

$-5.198603$

$-6.321465$

$-5.377038$

$-7.577622$

$-6.620250$

$-7.722874$

$-1.359757$

$-2.139749$

$-0.868115$

$-2.418598$

$-1.128843$

$-1.908442$

4. 240792

6.916443

8.420042

7.190722

4.496674

$-2.646288$
$-2.913872$

2. 427711

3. 778858

4.578433

3. 722511

2.420017

4.074680

5.652919

$-2.303825$

4.152516

0.137890

1. 346821

0.829761

1.399015

1.531037

1.684847

1.486450

1.734840

1.529512

1. 640545

$-3.664025$

$-4.812636$

$-3.846665$

$-6.091934$

$-5.117286$

$-6.242191$

$-0.225808$

$-0.147165$

$-0.727603$

$-0.563818$

$-1.160804$

$-1.074662$

5.607504

6.318681

6.306961

7.673224

7.663783

8.346315

1.746457

1.831198

1.642942

1. 455543

1.360363

5.693545
3.281150

1.168496

1.014776

0.888124

0.896384

1.075841

1.052089

0.785054

2. 617817

0.661725

0.408246

1. 511859

1. 222294

3. 423888

1. 467623

0.245574

2.616345

0.157317

2. 553078

1. 313725

3.208161

2. 420464

4.565647

2. 952371

5.120382

4.309996

$-0.080586$

0.746415

$-1.371862$

0.312369

$-1.824788$

$-0.977429$

0.476234

1. 394270

$-0.631721$

1. 227451

$-0.813498$

0.118793

$-0.899471$

$-1.037771$

1.226166

3.668196

3. 820274

2. 463272 


$$
\begin{aligned}
& -3.166288 \\
& 8.329579 \\
& 2.120655 \\
& -2.165736 \\
& -0.643734 \\
& -0.120945 \\
& -6.196296 \\
& -8.640229 \\
& -8.922883 \\
& -6.761357 \\
& -4.336715 \\
& -0.498060 \\
& -0.238669 \\
& 0.304816 \\
& 0.585882 \\
& 0.319830 \\
& -0.121843 \\
& -0.575815 \\
& 0.698226 \\
& 0.358707 \\
& \text { 2. } 030567 \\
& \text { 1.377697 } \\
& -0.684009 \\
& 3.039018 \\
& \text { 2. } 267279 \\
& \text { 2. } 726885 \\
& 1.121628 \\
& 4.077091 \\
& 3.817399 \\
& \text { 3. } 635892 \\
& \text { 3. } 851001 \\
& 4.796918 \\
& -1.870443 \\
& -0.430596 \\
& -0.109279 \\
& 4.892960 \\
& 4.460767 \\
& \text { 3. } 088175 \\
& \text { 2. } 155244 \\
& \text { 2. } 652780
\end{aligned}
$$




\begin{tabular}{|c|c|c|c|}
\hline $\mathrm{C}$ & 0.378135 & -3.880845 & -2.934353 \\
\hline C & 1.307347 & -4.367154 & -3.876483 \\
\hline O & 6.245665 & -1.623387 & -1.534131 \\
\hline $\mathrm{C}$ & 8.347984 & -0.682626 & -2.030614 \\
\hline $\mathrm{H}$ & 8.878123 & -0.106593 & -2.789357 \\
\hline $\mathrm{H}$ & 8.399703 & -0.158499 & -1.072146 \\
\hline $\mathrm{H}$ & 8.815618 & -1.663725 & -1.901915 \\
\hline C & 6.909142 & -0.849141 & -2.450119 \\
\hline O & 6.396287 & -0.383077 & -3.438338 \\
\hline C & -1.023094 & -4.422565 & -2.827479 \\
\hline $\mathrm{C}$ & -2.023898 & -3.270768 & -2.611654 \\
\hline $\mathrm{C}$ & -1.656862 & -5.286975 & -3.933451 \\
\hline $\mathrm{H}$ & -1.250422 & -6.302764 & -3.958746 \\
\hline C & -3.161207 & -5.258911 & -3.561192 \\
\hline $\mathrm{H}$ & -3.453051 & -6.134817 & -2.969161 \\
\hline C & -3.355613 & -4.013819 & -2.669547 \\
\hline O & -4.382873 & -3.710443 & -2.099719 \\
\hline C & -2.013663 & -2.292039 & -3.809792 \\
\hline $\mathrm{H}$ & -2.635337 & -1.424599 & -3.598201 \\
\hline $\mathrm{H}$ & -0.995904 & -1.946635 & -3.993452 \\
\hline $\mathrm{H}$ & -2.376455 & -2.765963 & -4.728970 \\
\hline $\mathrm{H}$ & -3.837504 & -5.222953 & -4.421533 \\
\hline $\mathrm{H}$ & -1.486954 & -4.843027 & -4.920054 \\
\hline C & -1.720813 & -2.596682 & -1.277852 \\
\hline $\mathrm{C}$ & -0.224614 & -2.347519 & -1.039934 \\
\hline $\mathrm{H}$ & -2.097128 & -3.233791 & -0.469377 \\
\hline $\mathrm{H}$ & -2.265219 & -1.654386 & -1.210982 \\
\hline $\mathrm{H}$ & 0.057570 & -2.530026 & 0.000042 \\
\hline $\mathrm{H}$ & 0.978132 & -5.093429 & -4.614064 \\
\hline $\mathrm{H}$ & 3.324546 & -4.323711 & -4.605137 \\
\hline & -1.046234 & -5.028660 & -1.903931 \\
\hline
\end{tabular}

\section{Structure: ${ }^{3}$ INT8a}

$\mathrm{C}$
$\mathrm{C}$
$\mathrm{C}$
$\mathrm{C}$
$\mathrm{N}$
$\mathrm{H}$
$\mathrm{H}$
$\mathrm{C}$
$\mathrm{C}$
$\mathrm{C}$

$$
\begin{array}{rrr}
0.155237 & -1.951361 & 2.416388 \\
0.094123 & -3.390837 & 2.477259 \\
-0.963238 & -3.779211 & 1.712912 \\
-1.585194 & -2.580581 & 1.209012 \\
-0.885716 & -1.485633 & 1.651748 \\
0.799211 & -4.020014 & 2.999954 \\
-1.308316 & -4.784428 & 1.520605 \\
-4.135805 & 0.729399 & 0.183574 \\
-5.241643 & -0.056806 & -0.304358 \\
-4.827124 & -1.349872 & -0.342739
\end{array}
$$




$$
\begin{array}{rrr}
-3.489616 & -1.383900 & 0.197210 \\
-3.097214 & -0.104874 & 0.508715 \\
-6.197557 & 0.338686 & -0.613090 \\
-5.384869 & -2.209550 & -0.682703 \\
1.114572 & 2.171326 & 1.705910 \\
2.387913 & 2.174730 & 2.378720 \\
2.525982 & 0.960891 & 2.979160 \\
1.365975 & 0.180188 & 2.634904 \\
0.512928 & 0.947685 & 1.874289 \\
3.071340 & 3.011007 & 2.408424 \\
3.349338 & 0.614172 & 3.585797 \\
-0.776425 & 3.345941 & 0.656753 \\
-1.433166 & 4.548336 & 0.212706 \\
-2.751097 & 4.250285 & 0.061401 \\
-2.907436 & 2.847249 & 0.359090 \\
-1.694390 & 2.325489 & 0.729514 \\
-0.942182 & 5.498528 & 0.060297 \\
-3.547546 & 4.904614 & -0.261183 \\
1.201911 & -1.177012 & 2.923596 \\
-4.085612 & 2.123681 & 0.166032 \\
-2.770788 & -2.550253 & 0.468815 \\
0.552431 & 3.278739 & 1.066148 \\
-1.307746 & 0.428793 & 1.193065 \\
-2.178461 & 0.741822 & 3.299786 \\
1.417469 & 4.478179 & 0.867087 \\
2.080085 & 4.681878 & -0.345890 \\
1.621864 & 5.418687 & 1.879562 \\
2.934652 & 5.763582 & -0.543816 \\
2.463944 & 6.515280 & 1.701648 \\
3.124965 & 6.684082 & 0.485206 \\
2.260453 & -1.874680 & 3.705207 \\
3.543937 & -2.074240 & 3.184376 \\
1.995364 & -2.391741 & 4.977502 \\
4.531084 & -2.745612 & 3.901337 \\
2.965535 & -3.073225 & 5.709589 \\
4.238178 & -3.247447 & 5.168459 \\
-3.332351 & -3.851415 & 0.005792 \\
-4.483207 & -4.408119 & 0.572845 \\
-2.724723 & -4.566159 & -1.030437 \\
-5.022614 & -5.609195 & 0.115589 \\
-3.250914 & -5.760030 & -1.514256 \\
-4.401798 & -6.287887 & -0.931938 \\
-5.307289 & 2.886251 & -0.213459 \\
-5.960815 & 3.695565 & 0.721340
\end{array}
$$


C

C

C

C

F

F

$\mathrm{F}$

F

$\mathrm{F}$

F

F

F

F

F

$\mathrm{F}$

F

F

F

F

F

F

F

F

F

$\mathrm{N}$

S

C

C

C

C

$\mathrm{H}$

C

$\mathrm{H}$

C

$\mathrm{H}$

$\mathrm{H}$

C

$\mathrm{H}$

$\mathrm{H}$

$\mathrm{H}$

O

O

C

C
$-5.820420$

$-7.088366$

$-6.951081$

$-7.584018$

1. 925449

3. 586350

3. 943104

2. 646773

1.012977

$-5.497900$

$-7.696581$

$-8.665283$

$-7.426881$

$-5.229046$

$-5.104725$

$-6.124002$

$-4.909159$

$-2.655916$

$-1.595751$

3.853907

5.750496

5.175562

2.685934

0.780478

$-0.557577$

$-0.876998$

$-0.051134$

$-0.558364$

1.145708

0.160539

$-1.480518$

1.846916

1.526667

1. 379710

$-0.199348$

2. 786241

2.166162

3. 223639

2. 091949

1.781028

$-2.328787$

$-0.173051$

2.096654

1. 751502
2.856963

4.441380

3.589723

4.384899

3.804308

5.910885

7.722981

7.399580

5.266941

3.772208

5.205751

5.092869

3.542115

2.108951

$-3.776631$

$-6.115580$

$-7.437440$

$-6.405776$

$-4.101070$

$-1.610724$

$-2.918699$

$-3.895394$

$-3.556712$

$-2.244896$

0.087980

0.838041

$-0.347569$

$-1.648117$

$-0.007224$

$-2.625780$

$-1.892325$

$-0.998388$

1.004790

$-2.318560$

$-3.649625$

$-0.747278$

$-3.400592$

$-3.129708$

$-4.341800$

$-3.575683$

0.796393

2.126864

$-4.010127$

$-2.739353$
$-1.514788$

0.385089

$-1.870408$

$-0.916420$

$-1.343482$

$-1.704383$

0.309189

2. 686775

3. 061065

1.974378

1.297794

$-1.249460$

$-3.119030$

$-2.450671$

1. 575016

0.676360

$-1.378495$

$-2.524514$

$-1.592849$

1.966377

3.380375

5.862645

6.923665

5.516775

$-0.511460$

$-1.975556$

$-3.032697$

$-3.092708$

$-3.660613$

$-3.770690$

$-2.579278$

$-4.346920$

$-3.580809$

$-4.397431$

$-3.778313$

$-4.832861$

$-5.095209$

$-5.183678$

$-4.543175$

$-6.108690$

$-2.192757$

$-2.098330$

$-1.025613$

$-0.646139$ 
C

C

C

C

$\mathrm{H}$

C

C

$\mathrm{H}$

$\mathrm{H}$

C

C

O

C

$\mathrm{H}$

$\mathrm{H}$

$\mathrm{H}$

C

O

C

C

C

$\mathrm{H}$

C

$\mathrm{H}$

C

O

C

$\mathrm{H}$

$\mathrm{H}$

$\mathrm{H}$

$\mathrm{H}$

$\mathrm{H}$

C

C

$\mathrm{H}$

$\mathrm{H}$

$\mathrm{H}$

$\mathrm{H}$

$\mathrm{H}$

$\mathrm{H}$

$\mathrm{H}$

\begin{tabular}{rrr}
2.646746 & -1.671559 & -0.898713 \\
3.896776 & -1.903168 & -1.578693 \\
4.184135 & -3.244142 & -1.959357 \\
3.315448 & -4.277022 & -1.684899 \\
0.797852 & -2.550328 & -0.173097 \\
2.388838 & -0.344723 & -0.545722 \\
4.780687 & -0.802833 & -1.841698 \\
5.111179 & -3.470293 & -2.473658 \\
3.551209 & -5.293695 & -1.977667 \\
4.432800 & 0.480370 & -1.427123 \\
3.187486 & 0.721609 & -0.771908 \\
1.239434 & -5.053642 & -0.666115 \\
-0.039647 & -6.999286 & -0.986718 \\
-0.662428 & -7.484523 & -1.735919 \\
0.658659 & -7.728901 & -0.561130 \\
-0.653304 & -6.614169 & -0.168066 \\
0.748297 & -5.886490 & -1.630656 \\
0.953865 & -5.757623 & -2.814058 \\
5.406259 & 1.608243 & -1.656321 \\
6.164232 & 1.437196 & -2.988314 \\
4.949574 & 3.077980 & -1.654581 \\
4.697112 & 3.433369 & -0.650715 \\
6.173228 & 3.811453 & -2.249804 \\
6.845387 & 4.188662 & -1.468910 \\
6.950196 & 2.749110 & -3.054281 \\
8.000394 & 2.924759 & -3.631773 \\
5.229037 & 1.433347 & -4.222746 \\
5.827142 & 1.318369 & -5.132617 \\
4.510208 & 0.611085 & -4.165272 \\
4.657036 & 2.362727 & -4.314071 \\
5.916292 & 4.667847 & -2.881019 \\
4.062511 & 3.216681 & -2.276370 \\
6.979287 & 0.150179 & -2.901359 \\
6.053276 & -1.066796 & -2.646581 \\
7.700459 & 0.248603 & -2.080593 \\
7.565043 & -0.009738 & -3.812923 \\
5.640775 & -1.856490 & -2.161621 \\
\hline .744042 & -1.487929 & -3.613746 \\
0.793766 & 1.729660 & -0.506583 \\
\hline .0 .032594 & -0.431948 \\
\hline .565 & 1.529134 & -0.863007
\end{tabular}

\section{Structure: ${ }^{5}$ INT8a}




\begin{tabular}{|c|c|c|c|}
\hline $\mathrm{C}$ & 0.232576 & -1.860894 & 2.368203 \\
\hline C & 0.216359 & -3.302173 & 2.424953 \\
\hline C & -0.840371 & -3.721988 & 1.675767 \\
\hline C & -1.507267 & -2.541461 & 1.187081 \\
\hline $\mathrm{N}$ & -0.834409 & -1.426745 & 1.620888 \\
\hline $\mathrm{H}$ & 0.949377 & -3.910652 & 2.933660 \\
\hline $\mathrm{H}$ & -1.151459 & -4.737037 & 1.476785 \\
\hline C & -4.181846 & 0.692507 & 0.234531 \\
\hline $\mathrm{C}$ & -5.272431 & -0.126728 & -0.231902 \\
\hline C & -4.817075 & -1.405671 & -0.288696 \\
\hline C & -3.466503 & -1.399124 & 0.218146 \\
\hline $\mathrm{N}$ & -3.108025 & -0.109363 & 0.527628 \\
\hline $\mathrm{H}$ & -6.248350 & 0.239046 & -0.513584 \\
\hline $\mathrm{H}$ & -5.355063 & -2.281456 & -0.619646 \\
\hline $\mathrm{C}$ & 1.057397 & 2.279092 & 1.628985 \\
\hline $\mathrm{C}$ & 2.341676 & 2.327514 & 2.280583 \\
\hline C & 2.523011 & 1.125369 & 2.894684 \\
\hline C & 1.380141 & 0.307407 & 2.577014 \\
\hline $\mathrm{N}$ & 0.494216 & 1.043557 & 1.825197 \\
\hline $\mathrm{H}$ & 2.996796 & 3.186968 & 2.293216 \\
\hline $\mathrm{H}$ & 3.364811 & 0.809751 & 3.492958 \\
\hline $\mathrm{C}$ & -0.894434 & 3.401328 & 0.633919 \\
\hline $\mathrm{C}$ & -1.595613 & 4.584431 & 0.205892 \\
\hline $\mathrm{C}$ & -2.909909 & 4.252866 & 0.100699 \\
\hline C & -3.019992 & 2.846509 & 0.404147 \\
\hline $\mathrm{N}$ & -1.783976 & 2.358513 & 0.741641 \\
\hline $\mathrm{H}$ & -1.134762 & 5.545273 & 0.028904 \\
\hline $\mathrm{H}$ & -3.732481 & 4.885516 & -0.198838 \\
\hline $\mathrm{C}$ & 1.259876 & -1.054615 & 2.868061 \\
\hline $\mathrm{C}$ & -4.178817 & 2.087717 & 0.230954 \\
\hline $\mathrm{C}$ & -2.706155 & -2.543081 & 0.468404 \\
\hline $\mathrm{C}$ & 0.450043 & 3.364432 & 0.990993 \\
\hline $\mathrm{Mn}$ & -1.327647 & 0.472948 & 1.181993 \\
\hline $\mathrm{Cl}$ & -2.144807 & 0.747471 & 3.312566 \\
\hline $\mathrm{C}$ & 1.291517 & 4.565289 & 0.719885 \\
\hline $\mathrm{C}$ & 2.094155 & 4.613836 & -0.422815 \\
\hline $\mathrm{C}$ & 1.331575 & 5.661254 & 1.584050 \\
\hline C & 2.928959 & 5.695675 & -0.689226 \\
\hline $\mathrm{C}$ & 2.150401 & 6.761508 & 1.332888 \\
\hline $\mathrm{C}$ & 2.955240 & 6.773838 & 0.194025 \\
\hline C & 2.339512 & -1.719664 & 3.649441 \\
\hline $\mathrm{C}$ & 3.631864 & -1.878096 & 3.136327 \\
\hline $\mathrm{C}$ & 2.083910 & -2.248815 & 4.919198 \\
\hline $\mathrm{C}$ & 4.635125 & -2.520379 & 3.857780 \\
\hline
\end{tabular}




\begin{tabular}{|c|c|c|c|}
\hline C & 3.070298 & -2.901840 & 5.655337 \\
\hline $\mathrm{C}$ & 4.350998 & -3.034901 & 5.121624 \\
\hline $\mathrm{C}$ & -3.240716 & -3.854110 & 0.001083 \\
\hline C & -4.340232 & -4.467615 & 0.608920 \\
\hline C & -2.673037 & -4.506520 & -1.096091 \\
\hline C & -4.869446 & -5.666548 & 0.134095 \\
\hline $\mathrm{C}$ & -3.194397 & -5.693526 & -1.601621 \\
\hline $\mathrm{C}$ & -4.292120 & -6.281527 & -0.976051 \\
\hline $\mathrm{C}$ & -5.431658 & 2.813778 & -0.116436 \\
\hline $\mathrm{C}$ & -6.090281 & 3.595807 & 0.837774 \\
\hline $\mathrm{C}$ & -5.970236 & 2.778011 & -1.407268 \\
\hline C & -7.247026 & 4.308705 & 0.530329 \\
\hline $\mathrm{C}$ & -7.129833 & 3.478065 & -1.734216 \\
\hline C & -7.767176 & 4.246432 & -0.761323 \\
\hline $\mathrm{F}$ & 2.085915 & 3.588782 & -1.283449 \\
\hline $\mathrm{F}$ & 3.709370 & 5.702469 & -1.777933 \\
\hline $\mathrm{F}$ & 3.750571 & 7.816750 & -0.049557 \\
\hline $\mathrm{F}$ & 2.175540 & 7.797347 & 2.176878 \\
\hline $\mathrm{F}$ & 0.574292 & 5.666989 & 2.687033 \\
\hline $\mathrm{F}$ & -5.604610 & 3.677411 & 2.081815 \\
\hline $\mathrm{F}$ & -7.859532 & 5.047336 & 1.461124 \\
\hline $\mathrm{F}$ & -8.876193 & 4.923113 & -1.066934 \\
\hline $\mathrm{F}$ & -7.629217 & 3.425266 & -2.973366 \\
\hline $\mathrm{F}$ & -5.374798 & 2.055675 & -2.360711 \\
\hline $\mathrm{F}$ & -4.923935 & -3.891116 & 1.665266 \\
\hline $\mathrm{F}$ & -5.921692 & -6.229316 & 0.734245 \\
\hline $\mathrm{F}$ & -4.791548 & -7.427059 & -1.441727 \\
\hline $\mathrm{F}$ & -2.642909 & -6.274447 & -2.673777 \\
\hline $\mathrm{F}$ & -1.595668 & -3.982280 & -1.703781 \\
\hline $\mathrm{F}$ & 3.936366 & -1.403695 & 1.921252 \\
\hline $\mathrm{F}$ & 5.862337 & -2.653459 & 3.343135 \\
\hline $\mathrm{F}$ & 5.304046 & -3.655161 & 5.819691 \\
\hline $\mathrm{F}$ & 2.798410 & -3.397283 & 6.866323 \\
\hline $\mathrm{F}$ & 0.862125 & -2.141936 & 5.452081 \\
\hline $\mathrm{N}$ & -0.579537 & 0.178152 & -0.536686 \\
\hline S & -0.935636 & 0.927580 & -1.989896 \\
\hline $\mathrm{C}$ & -0.089025 & -0.229447 & -3.062711 \\
\hline $\mathrm{C}$ & -0.597780 & -1.525841 & -3.172438 \\
\hline $\mathrm{C}$ & 1.130299 & 0.121940 & -3.640325 \\
\hline $\mathrm{C}$ & 0.139936 & -2.488419 & -3.852384 \\
\hline $\mathrm{H}$ & -1.537762 & -1.778330 & -2.696805 \\
\hline $\mathrm{C}$ & 1.850232 & -0.853596 & -4.329868 \\
\hline $\mathrm{H}$ & 1.511961 & 1.129922 & -3.520513 \\
\hline $\mathrm{C}$ & 1.379240 & -2.169741 & -4.431871 \\
\hline
\end{tabular}


C

$\mathrm{H}$

C

C

$\mathrm{H}$

$\mathrm{H}$

C

C

O

C

$\mathrm{H}$

$\mathrm{H}$

$\mathrm{H}$

C

O

C

C

C

$\mathrm{H}$

C

$\mathrm{H}$

C

O

C

$\mathrm{H}$

$\mathrm{H}$

$\mathrm{H}$

$\mathrm{H}$

$\mathrm{H}$

C

C

\begin{tabular}{|c|c|c|}
\hline-0.222034 & -3.510773 & -3.898381 \\
\hline 2.807025 & -0.593863 & -4.775738 \\
\hline 2.181956 & -3.235206 & -5.136865 \\
\hline & -2.982594 & -5.156602 \\
\hline 2.06173 & -4.199607 & -4.635323 \\
\hline 1.84874 & -3.353951 & -6.17662 \\
\hline 0 & 0.85 & -2.1 \\
\hline & & -2.11 \\
\hline 2.016 & -4.092 & -1.15 \\
\hline .687 & -2.82 & -0.76 \\
\hline & -1.77 & -0.9 \\
\hline 39 & -2.02 & -1.57 \\
\hline & -3.36 & -1.97 \\
\hline & -4.37 & -1.7 \\
\hline 0 & $-2 \cdot 61$ & -0.3 \\
\hline & -0.4 & -0.5 \\
\hline & -0.9 & $-1 \cdot 7$ \\
\hline & -3.6 & -2 . \\
\hline & -5.3 & \\
\hline & 0.3 & $-1 \cdot 31$ \\
\hline & & -0 \\
\hline & & -0 . \\
\hline-0.09 & -7.0 & -1.2 \\
\hline & -7.5 & -2.0 \\
\hline & -7.8 & -0 \\
\hline-0.74 & -6.7 & \\
\hline 0 & -5.9 & -1 \\
\hline 0 & -5.7 & -3 \\
\hline & & \\
\hline 6 & 1 . & -2 . \\
\hline 5 & 2.9 & -1.4 \\
\hline & & -0.4 \\
\hline 6 & 3.6 & -1 \\
\hline 7 & 3.9 & $-1 \cdot 1$ \\
\hline 7.162 & 2.5 & -2.75 \\
\hline 8.244 & 2.72 & -3.27 \\
\hline 5 & 1.3 & -4.04 \\
\hline 6.101 & 1.22 & -4.926 \\
\hline & 0.53 & -4.045 \\
\hline 4.925 & 2.28 & -4.139 \\
\hline 6.181 & 4.51 & -2.578 \\
\hline & & -2.117 \\
\hline & -0.037587 & -2.67 \\
\hline & -1.2 & -2 . \\
\hline
\end{tabular}




$\begin{array}{lrrr}\mathrm{H} & 7.787522 & 0.014999 & -1.819265 \\ \mathrm{H} & 7.730685 & -0.190406 & -3.563027 \\ \mathrm{H} & 6.668360 & -2.051257 & -2.011177 \\ \mathrm{H} & 5.860556 & -1.616516 & -3.496686 \\ \mathrm{H} & 2.974793 & 1.594900 & -0.396461 \\ \mathrm{H} & 0.441321 & 0.043681 & -0.475031 \\ \mathrm{H} & 6.239354 & 1.314068 & -0.640087\end{array}$

\section{Structure: ${ }^{3}$ INT8b}

C

C

C

C

$\mathrm{N}$

$\mathrm{H}$

$\mathrm{H}$

C

C

C

C

$\mathrm{N}$

$\mathrm{H}$

$\mathrm{H}$

C

C

C

C

$\mathrm{N}$

$\mathrm{H}$

$\mathrm{H}$

C

C

C

C

$\mathrm{N}$

$\mathrm{H}$

$\mathrm{H}$

C

C

C

C

$\mathrm{Mn}$

$\mathrm{Cl}$ $\begin{array}{lll}1.976231 & -2.045419 & -1.666252\end{array}$

$3.356324-2.456249-1.790513$

$\begin{array}{lll}4.123358 & -1.348971 & -1.576429\end{array}$

$3.219677-0.254313-1.300584$

$1.927045-0.711976-1.337221$

$3.694883-3.450763-2.044998$

$5.200809-1.280013-1.617962$

$\begin{array}{lll}0.916592 & 3.387407 & -0.693729\end{array}$

$\begin{array}{llll}2.042711 & 4.270426 & -0.472479\end{array}$

$\begin{array}{lll}3.163801 & 3.511850 & -0.566204\end{array}$

$\begin{array}{lll}2.736151 & 2.156419 & -0.855120\end{array}$

$1.366959 \quad 2.115856 \quad-0.913384$

$\begin{array}{lll}1.978418 & 5.329474 & -0.270244\end{array}$

$\begin{array}{lll}4.188251 & 3.832123 & -0.443067\end{array}$

$\begin{array}{lll}-2.304353 & -1.257987 & -1.703385\end{array}$

$\begin{array}{lll}-2.727986 & -2.597654 & -2.057380\end{array}$

$\begin{array}{llll}-1.600341 & -3.352391 & -2.173841\end{array}$

$\begin{array}{lll}-0.478072 & -2.476923 & -1.899522\end{array}$

$-0.940829-1.220695-1.617901$

$\begin{array}{lll}-3.749693 & -2.912762 & -2.215586\end{array}$

$\begin{array}{lll}-1.532430 & -4.400122 & -2.430954\end{array}$

$\begin{array}{lll}-2.766000 & 1.152779 & -1.235067\end{array}$

$\begin{array}{lll}-3.672450 & 2.263224 & -1.023183\end{array}$

$\begin{array}{lll}-2.910812 & 3.367828 & -0.819479\end{array}$

$\begin{array}{lll}-1.527508 & 2.947949 & -0.887760\end{array}$

$\begin{array}{lll}-1.474361 & 1.599320 & -1.131650\end{array}$

$\begin{array}{lll}-4.750078 & 2.201396 & -1.003040\end{array}$

$\begin{array}{lll}-3.250394 & 4.370301 & -0.605326\end{array}$

$\begin{array}{llll}0.868989 & -2.876140 & -1.911630\end{array}$

$\begin{array}{lll}-0.427027 & 3.792530 & -0.690856\end{array}$

$\begin{array}{lll}3.609962 & 1.078015 & -1.064508\end{array}$

$\begin{array}{lll}-3.166291 & -0.167357 & -1.500531\end{array}$

$\begin{array}{lll}0.209127 & 0.482467 & -1.302870\end{array}$

$\begin{array}{llll}0.346336 & 0.826296 & -3.558283\end{array}$ 


\begin{tabular}{|c|c|c|c|}
\hline C & -4.631075 & -0.433158 & -1.551499 \\
\hline C & -5.255609 & -1.251038 & -0.605409 \\
\hline $\mathrm{C}$ & -5.455572 & 0.141975 & -2.526028 \\
\hline $\mathrm{C}$ & -6.629965 & -1.459195 & -0.594505 \\
\hline $\mathrm{C}$ & -6.835539 & -0.055446 & -2.539408 \\
\hline $\mathrm{C}$ & -7.426256 & -0.860086 & -1.567321 \\
\hline C & 1.152325 & -4.299989 & -2.260821 \\
\hline C & 1.583255 & -5.221846 & -1.304923 \\
\hline $\mathrm{C}$ & 1.001503 & -4.769932 & -3.570088 \\
\hline $\mathrm{C}$ & 1.860248 & -6.549139 & -1.617965 \\
\hline $\mathrm{C}$ & 1.265786 & -6.095569 & -3.911534 \\
\hline C & 1.700248 & -6.986404 & -2.931489 \\
\hline $\mathrm{C}$ & 5.069420 & 1.387251 & -1.123729 \\
\hline $\mathrm{C}$ & 5.591017 & 2.184280 & -2.151120 \\
\hline C & 5.980214 & 0.900366 & -0.182661 \\
\hline $\mathrm{C}$ & 6.947815 & 2.491464 & -2.229664 \\
\hline $\mathrm{C}$ & 7.341610 & 1.186131 & -0.240391 \\
\hline $\mathrm{C}$ & 7.825859 & 1.988879 & -1.271169 \\
\hline $\mathrm{C}$ & -0.716629 & 5.238340 & -0.462289 \\
\hline $\mathrm{C}$ & -1.219956 & 6.041007 & -1.492052 \\
\hline $\mathrm{C}$ & -0.513322 & 5.847736 & 0.779912 \\
\hline $\mathrm{C}$ & -1.514712 & 7.389050 & -1.299807 \\
\hline $\mathrm{C}$ & -0.797145 & 7.194953 & 0.994616 \\
\hline $\mathrm{C}$ & -1.299317 & 7.967403 & -0.050502 \\
\hline $\mathrm{F}$ & -4.525408 & -1.864670 & 0.345661 \\
\hline $\mathrm{F}$ & -7.194176 & -2.230807 & 0.348791 \\
\hline $\mathrm{F}$ & -8.746838 & -1.057528 & -1.567214 \\
\hline $\mathrm{F}$ & -7.594122 & 0.514782 & -3.480389 \\
\hline $\mathrm{F}$ & -4.926205 & 0.922720 & -3.472672 \\
\hline $\mathrm{F}$ & -1.440945 & 5.513882 & -2.701768 \\
\hline $\mathrm{F}$ & -1.997020 & 8.130869 & -2.303222 \\
\hline $\mathrm{F}$ & -1.577099 & 9.259379 & 0.145992 \\
\hline $\mathrm{F}$ & -0.595376 & 7.749394 & 2.196078 \\
\hline $\mathrm{F}$ & -0.035113 & 5.136051 & 1.808462 \\
\hline $\mathrm{F}$ & 4.780178 & 2.678697 & -3.092042 \\
\hline $\mathrm{F}$ & 7.414271 & 3.257754 & -3.221256 \\
\hline $\mathrm{F}$ & 9.128189 & 2.275569 & -1.340461 \\
\hline $\mathrm{F}$ & 8.178458 & 0.704497 & 0.683407 \\
\hline $\mathrm{F}$ & 5.551550 & 0.121351 & 0.825874 \\
\hline $\mathrm{F}$ & 1.736054 & -4.837088 & -0.019552 \\
\hline $\mathrm{F}$ & 2.268467 & -7.403041 & -0.671493 \\
\hline $\mathrm{F}$ & 1.958862 & -8.257212 & -3.247988 \\
\hline F & 1.111988 & -6.516929 & -5.170295 \\
\hline$F$ & 0.586394 & -3.940848 & -4.531324 \\
\hline
\end{tabular}




\begin{tabular}{|c|c|c|c|}
\hline $\mathrm{N}$ & 0.110831 & -0.002351 & 0.876028 \\
\hline S & -0.099967 & 0.999577 & 2.145036 \\
\hline $\mathrm{C}$ & -1.856697 & 1.346728 & 2.137778 \\
\hline C & -2.781010 & 0.309993 & 1.998494 \\
\hline C & -2.275642 & 2.644498 & 2.430887 \\
\hline C & -4.136922 & 0.582905 & 2.158660 \\
\hline $\mathrm{H}$ & -2.439573 & -0.689073 & 1.758248 \\
\hline $\mathrm{C}$ & -3.638517 & 2.901846 & 2.581185 \\
\hline $\mathrm{H}$ & -1.537630 & 3.432113 & 2.530451 \\
\hline $\mathrm{C}$ & -4.585423 & 1.879647 & 2.447756 \\
\hline $\mathrm{H}$ & -4.853468 & -0.226865 & 2.082449 \\
\hline $\mathrm{H}$ & -3.969439 & 3.914157 & 2.802442 \\
\hline $\mathrm{C}$ & -6.064444 & 2.159487 & 2.569145 \\
\hline $\mathrm{H}$ & -6.259390 & 3.076700 & 3.134648 \\
\hline $\mathrm{H}$ & -6.521381 & 2.283316 & 1.577287 \\
\hline $\mathrm{H}$ & -6.586748 & 1.333449 & 3.064651 \\
\hline O & 0.162931 & 0.213076 & 3.391771 \\
\hline O & 0.606640 & 2.296583 & 2.079324 \\
\hline $\mathrm{H}$ & 1.024886 & -0.433073 & 1.035302 \\
\hline $\mathrm{C}$ & -2.932152 & -3.406458 & 2.536688 \\
\hline C & -2.448395 & -2.980583 & 3.756107 \\
\hline $\mathrm{C}$ & -1.072163 & -2.662602 & 3.879171 \\
\hline $\mathrm{C}$ & -0.190982 & -2.763294 & 2.758870 \\
\hline $\mathrm{C}$ & -0.742612 & -3.139176 & 1.523954 \\
\hline $\mathrm{C}$ & -2.084355 & -3.464531 & 1.416193 \\
\hline $\mathrm{H}$ & -3.102545 & -2.891333 & 4.613691 \\
\hline $\mathrm{C}$ & -0.549159 & -2.266038 & 5.132108 \\
\hline C & 1.228171 & -2.468137 & 2.960036 \\
\hline $\mathrm{H}$ & -0.134015 & -3.196077 & 0.633252 \\
\hline $\mathrm{H}$ & -2.500450 & -3.756703 & 0.459480 \\
\hline C & 1.703102 & -2.153721 & 4.287963 \\
\hline C & 0.806260 & -2.043237 & 5.341544 \\
\hline 0 & -4.216681 & -3.882784 & 2.336175 \\
\hline C & -6.549412 & -4.059514 & 2.571398 \\
\hline $\mathrm{H}$ & -7.431484 & -3.486354 & 2.856208 \\
\hline $\mathrm{H}$ & -6.585625 & -4.303192 & 1.508044 \\
\hline $\mathrm{H}$ & -6.518872 & -4.999121 & 3.134434 \\
\hline $\mathrm{C}$ & -5.314419 & -3.257961 & 2.882628 \\
\hline O & -5.257693 & -2.241129 & 3.527715 \\
\hline $\mathrm{C}$ & 3.185229 & -2.067241 & 4.473027 \\
\hline $\mathrm{C}$ & 3.869296 & -1.312168 & 3.310434 \\
\hline $\mathrm{C}$ & 3.815481 & -1.471802 & 5.745419 \\
\hline $\mathrm{H}$ & 3.693221 & -2.120729 & 6.617807 \\
\hline C & 5.301089 & -1.307610 & 5.332040 \\
\hline
\end{tabular}




$\begin{array}{lrrr}\mathrm{H} & 5.928667 & -2.128222 & 5.697104 \\ \mathrm{C} & 5.320354 & -1.351779 & 3.788509 \\ \mathrm{O} & 6.307220 & -1.446229 & 3.095932 \\ \mathrm{C} & 3.449640 & 0.179108 & 3.240315 \\ \mathrm{H} & 3.865014 & 0.632220 & 2.339013 \\ \mathrm{H} & 2.363822 & 0.288852 & 3.231945 \\ \mathrm{H} & 3.824562 & 0.748911 & 4.096748 \\ \mathrm{H} & 5.756762 & -0.378714 & 5.689717 \\ \mathrm{H} & 3.356990 & -0.506773 & 5.982085 \\ \mathrm{C} & 3.557085 & -2.042280 & 2.005508 \\ \mathrm{C} & 2.127695 & -2.451566 & 1.912630 \\ \mathrm{H} & 4.171941 & -2.956651 & 1.912615 \\ \mathrm{H} & 3.819256 & -1.453424 & 1.119937 \\ \mathrm{H} & 1.799456 & -2.749533 & 0.924081 \\ \mathrm{H} & 1.164458 & -1.781048 & 6.330918 \\ \mathrm{H} & -1.239555 & -2.158500 & 5.965320 \\ \mathrm{H} & 3.539594 & -3.113968 & 4.404675\end{array}$

\section{Structure: ${ }^{5}$ INT $8 b$}

$\begin{array}{lrrr}\mathrm{C} & 2.016976 & -2.164799 & -1.561889 \\ \mathrm{C} & 3.392631 & -2.580057 & -1.772730 \\ \mathrm{C} & 4.171954 & -1.480170 & -1.556090 \\ \mathrm{C} & 3.275727 & -0.393874 & -1.193612 \\ \mathrm{~N} & 1.999571 & -0.863487 & -1.160222 \\ \mathrm{H} & 3.716257 & -3.560262 & -2.095138 \\ \mathrm{H} & 5.244878 & -1.407564 & -1.665582 \\ \mathrm{C} & 0.927062 & 3.325935 & -0.716872 \\ \mathrm{C} & 2.071674 & 4.182255 & -0.499430 \\ \mathrm{C} & 3.180554 & 3.399860 & -0.550752 \\ \mathrm{C} & 2.745340 & 2.043342 & -0.811397 \\ \mathrm{~N} & 1.371296 & 2.038381 & -0.890399 \\ \mathrm{H} & 2.029865 & 5.247872 & -0.329575 \\ \mathrm{H} & 4.206304 & 3.709611 & -0.414388 \\ \mathrm{C} & -2.297120 & -1.202414 & -1.664797 \\ \mathrm{C} & -2.728408 & -2.538380 & -2.015045 \\ \mathrm{C} & -1.613167 & -3.314712 & -2.114752 \\ \mathrm{C} & -0.473059 & -2.470011 & -1.830039 \\ \mathrm{~N} & -0.926227 & -1.200756 & -1.560190 \\ \mathrm{H} & -3.751356 & -2.839815 & -2.189265 \\ \mathrm{H} & -1.567791 & -4.365230 & -2.364352 \\ \mathrm{C} & -2.817014 & 1.230969 & -1.261395 \\ \mathrm{C} & -3.718162 & 2.359481 & -1.065999 \\ \mathrm{C} & -2.938480 & 3.456624 & -0.875209\end{array}$




\begin{tabular}{|c|c|c|c|}
\hline C & -1.555853 & 3.005357 & -0.937239 \\
\hline N & -1.537438 & 1.668629 & -1.172369 \\
\hline $\mathrm{H}$ & -4.797393 & 2.315587 & -1.048109 \\
\hline $\mathrm{H}$ & -3.264196 & 4.467512 & -0.676367 \\
\hline $\mathrm{C}$ & 0.863593 & -2.927831 & -1.830991 \\
\hline C & -0.406215 & 3.788501 & -0.740801 \\
\hline C & 3.631090 & 0.958042 & -0.991587 \\
\hline $\mathrm{C}$ & -3.177262 & -0.110417 & -1.495652 \\
\hline $\mathrm{Mn}$ & 0.217532 & 0.442669 & -1.225350 \\
\hline $\mathrm{Cl}$ & 0.520975 & 0.685567 & -3.545306 \\
\hline $\mathrm{C}$ & -4.637866 & -0.399765 & -1.543920 \\
\hline $\mathrm{C}$ & -5.254609 & -1.208418 & -0.584556 \\
\hline $\mathrm{C}$ & -5.470537 & 0.162253 & -2.519383 \\
\hline $\mathrm{C}$ & -6.627693 & -1.422718 & -0.564641 \\
\hline $\mathrm{C}$ & -6.849653 & -0.041922 & -2.523767 \\
\hline $\mathrm{C}$ & -7.431862 & -0.838286 & -1.539973 \\
\hline $\mathrm{C}$ & 1.090255 & -4.352675 & -2.214097 \\
\hline $\mathrm{C}$ & 1.528544 & -5.297380 & -1.283864 \\
\hline $\mathrm{C}$ & 0.901515 & -4.798198 & -3.527402 \\
\hline $\mathrm{C}$ & 1.773248 & -6.624364 & -1.623487 \\
\hline C & 1.133215 & -6.122880 & -3.894926 \\
\hline $\mathrm{C}$ & 1.574200 & -7.037192 & -2.939568 \\
\hline $\mathrm{C}$ & 5.084455 & 1.286079 & -1.088763 \\
\hline $\mathrm{C}$ & 5.575456 & 2.092225 & -2.125344 \\
\hline $\mathrm{C}$ & 6.026431 & 0.782743 & -0.186743 \\
\hline $\mathrm{C}$ & 6.929420 & 2.398900 & -2.243344 \\
\hline $\mathrm{C}$ & 7.385545 & 1.068020 & -0.285184 \\
\hline $\mathrm{C}$ & 7.837534 & 1.883379 & -1.320545 \\
\hline $\mathrm{C}$ & -0.634594 & 5.249189 & -0.533855 \\
\hline $\mathrm{C}$ & -1.106223 & 6.052168 & -1.578537 \\
\hline $\mathrm{C}$ & -0.423869 & 5.869957 & 0.701701 \\
\hline $\mathrm{C}$ & -1.358626 & 7.411855 & -1.408542 \\
\hline $\mathrm{C}$ & -0.665676 & 7.228629 & 0.894076 \\
\hline C & -1.134261 & 8.001481 & -0.166239 \\
\hline $\mathrm{F}$ & -4.517486 & -1.804750 & 0.372043 \\
\hline $\mathrm{F}$ & -7.183648 & -2.184857 & 0.391340 \\
\hline $\mathrm{F}$ & -8.751527 & -1.041736 & -1.530673 \\
\hline $\mathrm{F}$ & -7.615410 & 0.514425 & -3.467237 \\
\hline $\mathrm{F}$ & -4.950310 & 0.935140 & -3.477485 \\
\hline $\mathrm{F}$ & -1.335420 & 5.515121 & -2.782160 \\
\hline $\mathrm{F}$ & -1.809595 & 8.153522 & -2.426489 \\
\hline $\mathrm{F}$ & -1.371566 & 9.304483 & 0.009428 \\
\hline $\mathrm{F}$ & -0.456916 & 7.793784 & 2.089458 \\
\hline $\mathrm{F}$ & 0.019411 & 5.158895 & 1.746117 \\
\hline
\end{tabular}




\begin{tabular}{|c|c|c|c|}
\hline $\mathrm{F}$ & 4.739106 & 2.594395 & -3.038724 \\
\hline $\mathrm{F}$ & 7.364959 & 3.175207 & -3.241154 \\
\hline $\mathrm{F}$ & 9.137379 & 2.169278 & -1.428577 \\
\hline $\mathrm{F}$ & 8.251850 & 0.572560 & 0.603790 \\
\hline $\mathrm{F}$ & 5.631370 & -0.010871 & 0.824076 \\
\hline $\mathrm{F}$ & 1.716443 & -4.936010 & 0.003963 \\
\hline $\mathrm{F}$ & 2.186956 & -7.500753 & -0.700238 \\
\hline $\mathrm{F}$ & 1.801233 & -8.307277 & -3.282329 \\
\hline F & 0.943781 & -6.521189 & -5.156322 \\
\hline $\mathrm{F}$ & 0.482726 & -3.946453 & -4.466337 \\
\hline $\mathrm{N}$ & 0.034929 & 0.063212 & 0.823025 \\
\hline S & -0.155243 & 1.048165 & 2.104330 \\
\hline $\mathrm{C}$ & -1.906226 & 1.425845 & 2.116823 \\
\hline $\mathrm{C}$ & -2.842224 & 0.397821 & 1.990049 \\
\hline $\mathrm{C}$ & -2.309391 & 2.731267 & 2.396533 \\
\hline $\mathrm{C}$ & -4.194531 & 0.686361 & 2.151156 \\
\hline $\mathrm{H}$ & -2.512516 & -0.607322 & 1.756638 \\
\hline $\mathrm{C}$ & -3.669308 & 3.004832 & 2.546462 \\
\hline $\mathrm{H}$ & -1.562271 & 3.511785 & 2.484535 \\
\hline $\mathrm{C}$ & -4.627706 & 1.991606 & 2.426662 \\
\hline $\mathrm{H}$ & -4.919677 & -0.116953 & 2.084040 \\
\hline $\mathrm{H}$ & -3.988977 & 4.023222 & 2.755878 \\
\hline $\mathrm{C}$ & -6.103035 & 2.290929 & 2.546094 \\
\hline $\mathrm{H}$ & -6.286476 & 3.210521 & 3.111599 \\
\hline $\mathrm{H}$ & -6.556332 & 2.421569 & 1.553496 \\
\hline $\mathrm{H}$ & -6.637492 & 1.472016 & 3.040383 \\
\hline O & 0.108843 & 0.241780 & 3.336385 \\
\hline O & 0.564869 & 2.335353 & 2.036580 \\
\hline $\mathrm{H}$ & 0.802715 & -0.565011 & 1.063759 \\
\hline $\mathrm{C}$ & -2.941339 & -3.360749 & 2.547146 \\
\hline C & -2.470545 & -2.896014 & 3.757862 \\
\hline C & -1.094275 & -2.585121 & 3.890181 \\
\hline $\mathrm{C}$ & -0.195148 & -2.738038 & 2.789638 \\
\hline C & -0.732373 & -3.155880 & 1.561886 \\
\hline $\mathrm{C}$ & -2.077071 & -3.466833 & 1.443299 \\
\hline $\mathrm{H}$ & -3.136062 & -2.768840 & 4.601662 \\
\hline $\mathrm{C}$ & -0.590869 & -2.137405 & 5.133641 \\
\hline $\mathrm{C}$ & 1.224358 & -2.443153 & 3.001725 \\
\hline $\mathrm{H}$ & -0.110403 & -3.256617 & 0.683791 \\
\hline $\mathrm{H}$ & -2.481931 & -3.786688 & 0.490575 \\
\hline $\mathrm{C}$ & 1.674382 & -2.058124 & 4.321081 \\
\hline $\mathrm{C}$ & 0.760657 & -1.904050 & 5.354059 \\
\hline O & -4.225631 & -3.834641 & 2.344264 \\
\hline $\mathrm{C}$ & -6.557394 & -4.017684 & 2.584778 \\
\hline
\end{tabular}




\begin{tabular}{|c|c|c|c|}
\hline $\mathrm{H}$ & -7.442533 & -3.440821 & 2.851705 \\
\hline $\mathrm{H}$ & -6.590700 & -4.291563 & 1.528669 \\
\hline $\mathrm{H}$ & -6.523324 & -4.940824 & 3.174307 \\
\hline $\mathrm{C}$ & -5.326800 & -3.202173 & 2.876080 \\
\hline O & -5.274750 & -2.170851 & 3.497889 \\
\hline $\mathrm{C}$ & 3.151655 & -1.952879 & 4.530959 \\
\hline $\mathrm{C}$ & 3.859919 & -1.281874 & 3.332721 \\
\hline C & 3.750118 & -1.265773 & 5.772593 \\
\hline $\mathrm{H}$ & 3.615032 & -1.855443 & 6.684204 \\
\hline C & 5.243092 & -1.115179 & 5.381111 \\
\hline $\mathrm{H}$ & 5.872971 & -1.893972 & 5.824898 \\
\hline $\mathrm{C}$ & 5.299352 & -1.280895 & 3.847033 \\
\hline 0 & 6.302568 & -1.433213 & 3.189459 \\
\hline $\mathrm{C}$ & 3.431868 & 0.195851 & 3.139720 \\
\hline $\mathrm{H}$ & 3.862527 & 0.583186 & 2.215533 \\
\hline $\mathrm{H}$ & 2.346123 & 0.295821 & 3.100033 \\
\hline $\mathrm{H}$ & 3.782087 & 0.832546 & 3.958651 \\
\hline $\mathrm{H}$ & 5.677211 & -0.153456 & 5.672937 \\
\hline $\mathrm{H}$ & 3.278431 & -0.291687 & 5.934249 \\
\hline C & 3.581479 & -2.111534 & 2.081054 \\
\hline $\mathrm{C}$ & 2.149507 & -2.509211 & 1.977764 \\
\hline $\mathrm{H}$ & 4.185104 & -3.038284 & 2.086035 \\
\hline $\mathrm{H}$ & 3.879699 & -1.600376 & 1.159704 \\
\hline $\mathrm{H}$ & 1.843237 & -2.875322 & 1.004986 \\
\hline $\mathrm{H}$ & 1.102633 & -1.595284 & 6.335619 \\
\hline $\mathrm{H}$ & -1.294601 & -1.992941 & 5.949865 \\
\hline $\mathrm{H}$ & 3.509652 & -3.000784 & 4.546161 \\
\hline
\end{tabular}

\section{Structure: ${ }^{3}$ TS9a}

$\mathrm{N}$

S

C

C

C

C

$\mathrm{H}$

C

$\mathrm{H}$

C

$\mathrm{H}$

$\mathrm{H}$

C

$\mathrm{H}$

$$
\begin{array}{rrr}
0.767388 & -0.447810 & -0.761403 \\
1.544722 & -1.232890 & -2.029034 \\
1.425310 & 0.039971 & -3.285781 \\
2.412664 & 1.025595 & -3.328426 \\
0.309713 & 0.092267 & -4.121947 \\
2.252790 & 2.102743 & -4.196698 \\
3.275597 & 0.951420 & -2.676885 \\
0.168458 & 1.178088 & -4.986330 \\
-0.431616 & -0.697382 & -4.080418 \\
1.118570 & 2.208102 & -5.016743 \\
3.001107 & 2.890161 & -4.210227 \\
-0.708554 & 1.239982 & -5.625055 \\
0.905608 & 3.444072 & -5.855977 \\
1.829752 & 3.755518 & -6.355742
\end{array}
$$


$\mathrm{H}$

$\mathrm{H}$

0

0

C

C

C

C

C

C

$\mathrm{H}$

C

C

$\mathrm{H}$

$\mathrm{H}$

C

C

O

C

$\mathrm{H}$

$\mathrm{H}$

$\mathrm{H}$

C

O

C

C

C

C

$\mathrm{H}$

C

O

C

$\mathrm{H}$

$\mathrm{H}$

$\mathrm{H}$

$\mathrm{H}$

H

C

C

$\mathrm{H}$

H

$\mathrm{H}$

$\mathrm{H}$

$\mathrm{H}$

$$
0.137617
$$

0.581298

2.970419

0.727032

$-0.922998$

$-0.659884$

$-1.715700$

$-3.057109$

$-3.243272$

$-2.208228$

0.336513

$-1.549881$

$-4.129288$

$-4.226562$

$-2.370433$

$-3.861551$

$-2.526643$

0.075468

1.454097

2.176208

0.816202

1.977325

0.598087

0.383877

$-4.994276$

$-6.316131$

$-4.903346$

$-6.367945$

$-6.544287$

$-7.238229$

$-8.438774$

$-6.262788$

$-7.231827$

$-5.488264$

$-6.050734$

$-6.691928$

$-4.553047$

$-6.675835$

$-5.552630$

$-6.810202$

$-7.627504$

$-5.590848$

$-5.773332$

$-2.310332$
3.289866

4.276912

$-1.447264$

$-2.388075$

4.376957

3.032397

2. 113499

2.581733

3.993258

4.872656

2.675623

0.727115

1.634444

4.395688

5.945101

0.268982

$-0.198833$

5.263850

7.165628

6.642060

7.768540

7.813959

6.178247

6.183993

$-0.715190$

$-0.141805$

$-2.133275$

$-2.624141$

$-3.209508$

$-1.352684$

$-1.328184$

0.174273

0.570801

0.914027

$-0.714740$

$-3.248532$

$-2.107497$

1.099844

2.162904

0.817293

1.525241

2.800401

2.827310

$-1.260898$
$-6.620179$

$-5.217485$

$-1.732216$

$-2.430772$

$-2.043289$

$-2.059672$

$-1.818659$

$-1.567075$

$-1.499862$

$-1.733449$

$-2.294334$

$-1.851281$

$-1.439565$

$-1.282453$

$-1.704548$

$-1.538719$

$-1.718027$

$-2.473571$

$-2.359017$

$-2.990527$

$-3.014341$

$-1.656306$

$-1.606225$

$-0.415435$

$-1.403464$

$-1.954463$

$-2.000101$

$-1.903750$

$-0.992575$

$-1.811940$

$-1.650690$

$-3.470860$

$-3.791804$

$-3.693349$

$-4.074340$

$-2.742948$

$-3.037277$

$-1.144415$

$-1.257778$

$-0.095542$

$-1.480590$

$-0.365084$

$-2.104279$

$-1.744145$ 


$$
\begin{array}{rrr}
0.603968 & 0.526489 & -1.003487 \\
-4.210098 & -2.769642 & -1.446840 \\
-5.152729 & -0.864158 & -0.324850 \\
-1.059228 & 1.441928 & 1.850866 \\
-1.219634 & 2.872366 & 1.819854 \\
-0.078573 & 3.401214 & 1.296923 \\
0.831953 & 2.302603 & 1.087035 \\
0.185165 & 1.117958 & 1.370558 \\
-2.120218 & 3.399334 & 2.099645 \\
0.119993 & 4.435926 & 1.062711 \\
3.912984 & -0.701394 & 1.152485 \\
5.035149 & 0.206061 & 1.121043 \\
4.534543 & 1.456802 & 0.926606 \\
3.096163 & 1.339680 & 0.898667 \\
2.753345 & 0.022585 & 1.051523 \\
6.071772 & -0.083733 & 1.207780 \\
5.081486 & 2.385285 & 0.845434 \\
-1.369037 & -2.655514 & 0.812092 \\
-2.764240 & -2.830563 & 1.142806 \\
-3.157991 & -1.715223 & 1.820093 \\
-2.034832 & -0.808612 & 1.829632 \\
-0.959481 & -1.421271 & 1.236801 \\
-3.344396 & -3.717358 & 0.930795 \\
-4.131858 & -1.507511 & 2.237014 \\
0.853843 & -3.610559 & 0.360732 \\
1.742994 & -4.714812 & 0.118443 \\
2.993729 & -4.311011 & 0.478470 \\
2.901611 & -2.930278 & 0.874010 \\
1.587843 & -2.535170 & 0.803937 \\
1.444960 & -5.682011 & -0.257005 \\
3.910156 & -4.880814 & 0.432355 \\
-2.067676 & 0.537939 & 2.206781 \\
3.996727 & -2.096452 & 1.120496 \\
2.201935 & 2.417367 & 0.829233 \\
-0.541743 & -3.656535 & 0.291895 \\
0.907307 & -0.713339 & 1.114919 \\
1.126738 & -0.977857 & 3.388331 \\
-1.207444 & -4.847065 & -0.301098 \\
-1.931408 & -4.722143 & -1.491933 \\
-1.183530 & -6.108178 & 0.301957 \\
-2.615741 & -5.795230 & -2.055573 \\
-1.844812 & -7.201838 & -0.252931 \\
-2.566851 & -7.042054 & -1.434711 \\
-3.213610 & 1.086923 & 2.983051
\end{array}
$$


C

C

C

C

C

C

C

C

C

C

C

C

C

C

C

C

C

F

F

F

F

F

F

F

F

F

F

F

F

F

F

F

F

$\mathrm{F}$

F

F

F
$-4.516340$

$-2.995417$

$-5.561920$

$-4.024355$

$-5.313876$

2.755679

2. 747937

3. 290825

3. 217170

3. 784603

3. 735390

5.339222

5.689260

6.278559

6.927839

7.525186

7.847943

$-2.002139$

$-3.315919$

$-3.213649$

$-1.804372$

$-0.515340$

4.820806

7.239294

9.038650

8.408288

5.992738

2. 262400

3.169155

4.147346

4.257299

3. 324587

$-4.797427$

$-6.789028$

$-6.302577$

$-3.783540$

$-1.772652$
1.172197

1.601773

1.730637

2.165088

2.230331

3.775167

4.784305

4.100228

6.067952

5.367157

6.358391

$-2.736039$

$-3.547669$

$-2.588332$

$-4.181597$

$-3.207829$

$-4.007993$

$-3.540486$

$-5.634292$

$-8.080409$

$-8.395851$

$-6.288055$

$-3.734939$

$-4.950126$

$-4.608249$

$-3.048238$

$-1.835234$

4.536233

7.018614

7.597054

5.649252

3.177201

0.716106

1.798056

2.770089

2.643951

1.567156
2.491133

4.269613

3.218852

5. 022245

4.495065

0.570369

1.539030

$-0.678016$

1.269383

$-0.965306$

0.010759

1.189758

2.273734

0.163222

2. 345925

0.218732

1. 313528

$-2.119708$

$-3.183240$

$-1.967261$

0.346566

1. 449161

3.273792

3. 394543

1. 372452

$-0.772940$

$-0.903790$

2. 759641

2. 204740

$-0.277088$

$-2.189069$

$-1.652083$

1.260867

2.687685

5.208926

6.245676

4.804241

\section{Structure: ${ }^{5}$ TS9a}

$\mathrm{N}$
$\mathrm{S}$
$\mathrm{C}$
$\mathrm{C}$

N

$S$

$\mathrm{C}$

C

17.782069

$-4.836414$

5.919739

$-4.121827$

4.641481

$-4.741186$

3.249915

17.832967

$-5.971094$

2.685057 


\begin{tabular}{|c|c|c|c|}
\hline $\mathrm{C}$ & 18.950063 & -4.023947 & 2.817395 \\
\hline C & 18.313279 & -6.503292 & 1.691910 \\
\hline $\mathrm{H}$ & 16.610077 & -6.498960 & 3.025971 \\
\hline $\mathrm{C}$ & 19.754042 & -4.572330 & 1.822674 \\
\hline $\mathrm{H}$ & 19.187785 & -3.069139 & 3.273039 \\
\hline C & 19.463426 & -5.825896 & 1.263186 \\
\hline $\mathrm{H}$ & 18.070474 & -7.471769 & 1.262140 \\
\hline $\mathrm{H}$ & 20.631671 & -4.027160 & 1.485307 \\
\hline$C$ & 20.398242 & -6.458195 & 0.261679 \\
\hline $\mathrm{H}$ & 19.856063 & -7.070254 & -0.466989 \\
\hline $\mathrm{H}$ & 20.975905 & -5.705067 & -0.284428 \\
\hline $\mathrm{H}$ & 21.110263 & -7.114490 & 0.778596 \\
\hline 0 & 15.528420 & -4.673997 & 4.568906 \\
\hline 0 & 17.088354 & -2.668760 & 4.713806 \\
\hline C & 20.252538 & -8.878350 & 3.741900 \\
\hline $\mathrm{C}$ & 19.764875 & -7.824224 & 4.472192 \\
\hline $\mathrm{C}$ & 20.588827 & -6.685635 & 4.690218 \\
\hline$C$ & 21.929188 & -6.643687 & 4.150492 \\
\hline $\mathrm{C}$ & 22.375327 & -7.779211 & 3.417278 \\
\hline $\mathrm{C}$ & 21.560116 & -8.868518 & 3.214150 \\
\hline $\mathrm{H}$ & 18.748801 & -7.841627 & 4.844758 \\
\hline $\mathrm{C}$ & 20.180267 & -5.537411 & 5.382717 \\
\hline $\mathrm{C}$ & 22.751009 & -5.483623 & 4.345490 \\
\hline $\mathrm{H}$ & 23.376286 & -7.794347 & 3.001961 \\
\hline $\mathrm{H}$ & 21.898276 & -9.728454 & 2.644748 \\
\hline $\mathrm{C}$ & 22.245366 & -4.388311 & 5.041389 \\
\hline $\mathrm{C}$ & 20.917621 & -4.415848 & 5.561605 \\
\hline O & 19.470237 & -9.987223 & 3.391403 \\
\hline $\mathrm{C}$ & 18.662751 & -12.179541 & 3.624203 \\
\hline $\mathrm{H}$ & 17.822266 & -11.927439 & 2.972495 \\
\hline $\mathrm{H}$ & 19.457732 & -12.589396 & 2.992317 \\
\hline $\mathrm{H}$ & 18.355573 & -12.922491 & 4.359706 \\
\hline C & 19.173794 & -10.943460 & 4.321981 \\
\hline O & 19.319671 & -10.803472 & 5.513250 \\
\hline C & 23.143757 & -3.196851 & 5.270562 \\
\hline $\mathrm{C}$ & 24.115696 & -2.977790 & 4.093137 \\
\hline $\mathrm{C}$ & 22.570713 & -1.795944 & 5.551133 \\
\hline $\mathrm{C}$ & 23.808592 & -0.884014 & 5.392095 \\
\hline $\mathrm{H}$ & 24.307959 & -0.689857 & 6.349615 \\
\hline $\mathrm{C}$ & 24.794912 & -1.670720 & 4.505066 \\
\hline 0 & 25.910088 & -1.313934 & 4.193834 \\
\hline C & 23.395172 & -2.679089 & 2.753615 \\
\hline $\mathrm{H}$ & 24.139435 & -2.545217 & 1.961654 \\
\hline $\mathrm{H}$ & 22.727448 & -3.500237 & 2.477251 \\
\hline
\end{tabular}




\begin{tabular}{|c|c|c|c|}
\hline $\mathrm{H}$ & 22.791613 & -1.766563 & 2.800551 \\
\hline $\mathrm{H}$ & 23.584555 & 0.091751 & 4.947574 \\
\hline $\mathrm{H}$ & 21.804894 & -1.536140 & 4.819470 \\
\hline C & 25.005599 & -4.210395 & 3.983165 \\
\hline C & 24.144664 & -5.467972 & 3.715336 \\
\hline $\mathrm{H}$ & 25.557518 & -4.328832 & 4.923700 \\
\hline $\mathrm{H}$ & 25.755200 & -4.091878 & 3.193133 \\
\hline $\mathrm{H}$ & 24.706732 & -6.351160 & 4.044528 \\
\hline $\mathrm{H}$ & 24.020458 & -5.590037 & 2.630026 \\
\hline $\mathrm{H}$ & 20.509036 & -3.547140 & 6.063865 \\
\hline $\mathrm{H}$ & 17.684993 & -5.841766 & 5.744534 \\
\hline $\mathrm{H}$ & 22.112943 & -1.726634 & 6.541131 \\
\hline $\mathrm{H}$ & 23.769952 & -3.448292 & 6.142717 \\
\hline C & 19.540347 & -6.490118 & 8.629450 \\
\hline $\mathrm{C}$ & 20.002022 & -7.847813 & 8.481532 \\
\hline C & 19.028784 & -8.542516 & 7.830466 \\
\hline $\mathrm{C}$ & 17.928033 & -7.631332 & 7.637341 \\
\hline $\mathrm{N}$ & 18.274394 & -6.386157 & 8.108080 \\
\hline $\mathrm{H}$ & 20.974435 & -8.206336 & 8.786227 \\
\hline $\mathrm{H}$ & 19.058871 & -9.566242 & 7.491233 \\
\hline C & 14.297117 & -5.307520 & 7.395967 \\
\hline C & 13.415096 & -6.416986 & 7.139619 \\
\hline $\mathrm{C}$ & 14.195695 & -7.515634 & 6.949639 \\
\hline $\mathrm{C}$ & 15.561954 & -7.106407 & 7.154825 \\
\hline $\mathrm{N}$ & 15.592185 & -5.765122 & 7.437606 \\
\hline $\mathrm{H}$ & 12.338499 & -6.354682 & 7.084554 \\
\hline $\mathrm{H}$ & 13.878685 & -8.524352 & 6.727992 \\
\hline C & 19.022663 & -2.303503 & 8.045353 \\
\hline $\mathrm{C}$ & 20.302844 & -1.879804 & 8.557055 \\
\hline $\mathrm{C}$ & 20.884365 & -2.972827 & 9.127561 \\
\hline $\mathrm{C}$ & 19.999518 & -4.088901 & 8.902499 \\
\hline $\mathrm{N}$ & 18.859900 & -3.639278 & 8.280586 \\
\hline $\mathrm{H}$ & 20.676993 & -0.866424 & 8.525539 \\
\hline $\mathrm{H}$ & 21.838103 & -3.025385 & 9.631347 \\
\hline C & 16.723119 & -1.776227 & 7.351514 \\
\hline $\mathrm{C}$ & 15.665639 & -0.847613 & 7.056602 \\
\hline C & 14.494324 & -1.535022 & 7.150067 \\
\hline $\mathrm{C}$ & 14.826448 & -2.908248 & 7.433955 \\
\hline $\mathrm{N}$ & 16.188285 & -3.023223 & 7.570768 \\
\hline $\mathrm{H}$ & 15.805697 & 0.199982 & 6.834522 \\
\hline $\mathrm{H}$ & 13.492589 & -1.161212 & 6.996860 \\
\hline $\mathrm{C}$ & 20.326462 & -5.433420 & 9.100515 \\
\hline $\mathrm{C}$ & 13.913101 & -3.965544 & 7.429765 \\
\hline & 16.663740 & -7.971297 & 7.144692 \\
\hline
\end{tabular}




\begin{tabular}{|c|c|c|c|}
\hline $\mathrm{C}$ & 18.068040 & -1.444644 & 7.489419 \\
\hline $\mathrm{Mn}$ & 17.208001 & -4.699864 & 7.874751 \\
\hline $\mathrm{Cl}$ & 16.671367 & -4.695527 & 10.142139 \\
\hline $\mathrm{C}$ & 18.572068 & -0.144404 & 6.973721 \\
\hline C & 19.367346 & -0.157471 & 5.822237 \\
\hline C & 18.360224 & 1.086547 & 7.594814 \\
\hline C & 19.975455 & 0.992919 & 5.332888 \\
\hline $\mathrm{C}$ & 18.934209 & 2.259874 & 7.105702 \\
\hline C & 19.753335 & 2.209150 & 5.978014 \\
\hline $\mathrm{C}$ & 21.633475 & -5.774845 & 9.726146 \\
\hline C & 22.854868 & -5.479806 & 9.112196 \\
\hline $\mathrm{C}$ & 21.682582 & -6.444692 & 10.954442 \\
\hline $\mathrm{C}$ & 24.074866 & -5.815582 & 9.691777 \\
\hline $\mathrm{C}$ & 22.891465 & -6.796431 & 11.551946 \\
\hline $\mathrm{C}$ & 24.091417 & -6.478617 & 10.917709 \\
\hline $\mathrm{C}$ & 16.446843 & -9.332214 & 6.588202 \\
\hline $\mathrm{C}$ & 16.482173 & -10.489274 & 7.369029 \\
\hline C & 16.177983 & -9.483511 & 5.224891 \\
\hline C & 16.260718 & -11.750190 & 6.819672 \\
\hline C & 15.928299 & -10.727048 & 4.658525 \\
\hline C & 15.975417 & -11.864311 & 5.462156 \\
\hline $\mathrm{C}$ & 12.467370 & -3.627512 & 7.322626 \\
\hline $\mathrm{C}$ & 11.797498 & -3.017793 & 8.388635 \\
\hline $\mathrm{C}$ & 11.737078 & -3.868365 & 6.153518 \\
\hline $\mathrm{C}$ & 10.451402 & -2.668651 & 8.306331 \\
\hline $\mathrm{C}$ & 10.388387 & -3.533179 & 6.052743 \\
\hline $\mathrm{C}$ & 9.746140 & -2.929915 & 7.132850 \\
\hline $\mathrm{F}$ & 19.582073 & -1.312577 & 5.177398 \\
\hline $\mathrm{F}$ & 20.778957 & 0.933796 & 4.262299 \\
\hline $\mathrm{F}$ & 20.324197 & 3.323457 & 5.517549 \\
\hline $\mathrm{F}$ & 18.720901 & 3.427243 & 7.720635 \\
\hline $\mathrm{F}$ & 17.598480 & 1.157247 & 8.694025 \\
\hline $\mathrm{F}$ & 12.454380 & -2.751140 & 9.522946 \\
\hline $\mathrm{F}$ & 9.834061 & -2.089757 & 9.341096 \\
\hline $\mathrm{F}$ & 8.455419 & -2.602442 & 7.042905 \\
\hline $\mathrm{F}$ & 9.709898 & -3.777428 & 4.926657 \\
\hline $\mathrm{F}$ & 12.329389 & -4.436420 & 5.098661 \\
\hline $\mathrm{F}$ & 16.737193 & -10.404031 & 8.678618 \\
\hline $\mathrm{F}$ & 16.316940 & -12.844945 & 7.582119 \\
\hline $\mathrm{F}$ & 15.791673 & -13.069911 & 4.916084 \\
\hline $\mathrm{F}$ & 15.700966 & -10.848840 & 3.342760 \\
\hline $\mathrm{F}$ & 16.182378 & -8.404074 & 4.423912 \\
\hline $\mathrm{F}$ & 22.875469 & -4.852457 & 7.926036 \\
\hline $\mathrm{F}$ & 25.223677 & -5.514946 & 9.075791 \\
\hline
\end{tabular}




\section{Structure: ${ }^{3} \mathrm{TS} 9 \mathrm{~b}$}

C

C

C

C

$\mathrm{N}$

$\mathrm{H}$

$\mathrm{H}$

C

C

C

C

$\mathrm{N}$

$\mathrm{H}$

$\mathrm{H}$

C

C

C

C

$\mathrm{N}$

$\mathrm{H}$

$\mathrm{H}$

C

C

C

C

$\mathrm{N}$

$\mathrm{H}$

$\mathrm{H}$

C

C

C

$\mathrm{C}$

$\mathrm{Mn}$

$\mathrm{Cl}$

C

C

C

C
2.274696

3. 688049

4.298283

3.262169

2. 041419

4.150034

5.351107

0.513317

1. 505258

2. 718092

2.475784

1.126283

1. 292831

3.689841

$-2.046485$

$-2.283625$

$-1.067052$

$-0.082047$

$-0.704584$

$-3.247480$

$-0.849249$

$-2.815478$

$-3.865438$

$-3.261691$

$-1.836008$

$-1.595492$

$-4.925385$

$-3.732680$

1.300690

$-0.868123$

3.485655

$-3.046337$

0.209315

0.245818

$-4.450382$

$-5.167507$

$-5.118647$

$-6.476567$
$-1.731434-1.644281$

$-1.943695-1.845507$

$-0.741209-1.656121$

$0.207018-1.316528$

$-0.420051-1.307372$

$-2.883241-2.113914$

$\begin{array}{ll}-0.517791 & -1.746544\end{array}$

$3.463645-0.603766$

$4.492114-0.369599$

$3.898198-0.481245$

$2.504328-0.799768$

$2.269883-0.856157$

$5.527582-0.147364$

$4.352761-0.355334$

$-1.539731-1.640362$

$-2.932071-1.961085$

$-3.540099-2.001140$

$-2.515708-1.725889$

$-1.320032-1.483535$

$-3.378125-2.159665$

$-4.573234-2.233456$

$0.788173-1.255553$

$1.770518-1.083904$

$2.948382-0.789004$

$2.701420-0.791710$

$1.375240-1.047048$

$1.573869-1.150020$

$3.896418-0.575511$

$-2.726056-1.791686$

$3.685332-0.574842$

$1.565647-1.040240$

$-0.560863-1.552865$

$0.494544-1.169931$

$0.842066-3.436306$

$-0.966157-1.848712$

$-1.867682-1.057434$

$-0.452187-2.969433$

$-2.238325-1.347857$ 


$$
\begin{array}{r}
-6.432976 \\
-7.116461 \\
1.772712 \\
1.874396 \\
2.128387 \\
2.301570 \\
2.565060 \\
2.649351 \\
4.897909 \\
5.348137 \\
5.840702 \\
6.664389 \\
7.162802 \\
7.574640
\end{array}
$$

$-1.348983$

$-1.926419$

$-1.256994$

$-2.400441$

$-1.720614$

$-2.294484$

$-4.600246$

$-7.131873$

$-8.374126$

$-7.038315$

$-4.497175$

$-2.046696$

$-2.950691$

$-2.744053$

$-1.622744$

$-0.710804$

4.506379

7.061836

8.838851

8.032798

5.484293

1. 552197

2. 384619

3. 063618

2. 899067

2.057981

0.287674

$-0.100611$

$-1.884268$

$-2.652020$
$-0.800544$

$-1.700166$

$-4.104807$

$-5.104049$

$-4.450668$

$-6.394339$

$-5.733379$

$-6.708236$

2.044202

2. 970621

1.579946

3.427504

2. 015390

2.946925

5.075918

5.831139

5.677176

7.124128

6.970354

7.695222

$-2.416874$

$-3.105981$

$-2.046664$

$-0.286791$

0.406950

5.308336

7.820463

8.934837

7.517432

5.013964

3. 446730

4.315726

3.376854

1. 552898

0.673689

$-4.836172$

$-7.325797$

$-7.940347$

$-6.036282$

$-3.537855$

0.114831

1.020783

1. 122364

0.014265
$-3.276286$

$-2.461040$

$-2.122756$

$-1.154118$

$-3.430510$

$-1.454308$

$-3.759531$

$-2.766688$

$-1.047800$

$-1.996847$

$-0.124757$

$-2.015242$

$-0.125005$

$-1.076012$

$-0.323077$

$-1.349822$

0.936108

$-1.138622$

1.169904

0.127928

0.037696

$-0.557647$

$-2.743910$

$-4.350696$

$-3.780400$

$-2.575537$

$-2.139739$

0.343523

2.387799

1. 962779

$-2.920453$

$-2.932503$

$-1.087473$

0.778611

0.802022

0.130498

$-0.496634$

$-3.070504$

$-5.017224$

$-4.402039$

0.807042

2.123081

2.110298

1. 757593 


\begin{tabular}{|c|c|c|c|}
\hline $\mathrm{C}$ & -2.480029 & 2.271293 & 2.630715 \\
\hline C & -4.027905 & 0.056218 & 1.949438 \\
\hline $\mathrm{H}$ & -2.173306 & -0.849537 & 1.316272 \\
\hline C & -3.861767 & 2.288370 & 2.830348 \\
\hline $\mathrm{H}$ & -1.866901 & 3.132526 & 2.869936 \\
\hline C & -4.652213 & 1.179071 & 2.508940 \\
\hline $\mathrm{H}$ & -4.627673 & -0.797900 & 1.659630 \\
\hline $\mathrm{H}$ & -4.329463 & 3.177767 & 3.246006 \\
\hline C & -6.141435 & 1.159915 & 2.753909 \\
\hline $\mathrm{H}$ & -6.493232 & 2.094209 & 3.202616 \\
\hline $\mathrm{H}$ & -6.696219 & 1.007098 & 1.818803 \\
\hline $\mathrm{H}$ & -6.404180 & 0.335195 & 3.428077 \\
\hline O & 0.246806 & 0.215554 & 3.331036 \\
\hline O & 0.434402 & 2.395888 & 2.121285 \\
\hline $\mathrm{H}$ & 1.268360 & -0.127505 & 0.962505 \\
\hline C & -2.997690 & -3.257766 & 2.814420 \\
\hline C & -2.409713 & -2.816284 & 3.982395 \\
\hline C & -1.009407 & -2.592641 & 4.004745 \\
\hline C & -0.214036 & -2.784102 & 2.834320 \\
\hline C & -0.854187 & -3.239247 & 1.670067 \\
\hline C & -2.215978 & -3.484255 & 1.664379 \\
\hline $\mathrm{H}$ & -2.999779 & -2.633014 & 4.869022 \\
\hline C & -0.373996 & -2.211886 & 5.210330 \\
\hline C & 1.215730 & -2.500470 & 2.915821 \\
\hline $\mathrm{H}$ & -0.296327 & -3.429512 & 0.764196 \\
\hline $\mathrm{H}$ & -2.704718 & -3.827324 & 0.760560 \\
\hline C & 1.813457 & -2.243687 & 4.203521 \\
\hline C & 1.006634 & -2.087101 & 5.322509 \\
\hline O & -4.337684 & -3.568715 & 2.660228 \\
\hline C & -6.672046 & -3.394024 & 2.804137 \\
\hline $\mathrm{H}$ & -7.476925 & -2.885439 & 3.335438 \\
\hline $\mathrm{H}$ & -6.737655 & -3.197311 & 1.730488 \\
\hline $\mathrm{H}$ & -6.756551 & -4.477154 & 2.941595 \\
\hline C & -5.345938 & -2.911710 & 3.326211 \\
\hline O & -5.169857 & -2.076436 & 4.178737 \\
\hline C & 3.308352 & -2.207695 & 4.247254 \\
\hline C & 3.878564 & -1.350109 & 3.092210 \\
\hline C & 4.086898 & -1.745620 & 5.492174 \\
\hline $\mathrm{H}$ & 4.046512 & -2.476998 & 6.304843 \\
\hline $\mathrm{C}$ & 5.521796 & -1.554157 & 4.935352 \\
\hline $\mathrm{H}$ & 6.167856 & -2.414295 & 5.143912 \\
\hline C & 5.371760 & -1.439152 & 3.403228 \\
\hline O & 6.273817 & -1.459809 & 2.597956 \\
\hline $\mathrm{C}$ & 3.471237 & 0.141277 & 3.202803 \\
\hline
\end{tabular}




\section{Structure: ${ }^{5}$ TS9b}

C

C

C

C

$\mathrm{N}$

$\mathrm{H}$

$\mathrm{H}$

C

C

C

C

$\mathrm{N}$

$\mathrm{H}$

$\mathrm{H}$

C

C

C

C

$\mathrm{N}$

$\mathrm{H}$

$\mathrm{H}$

C

C

C

C

$\mathrm{N}$

$\mathrm{H}$

$\mathrm{H}$
2.099946

3. 492606

4.222525

3.278549

2.019448

3.858985

5.292959

0.768433

1.870730

3.015780

2.644368

1.270685

1.776813

4.026960

$-2.244245$

$-2.609407$

$-1.461187$

$-0.365809$

$-0.877230$

$-3.613248$

$-1.364112$

$-2.862611$

$-3.813952$

$-3.087690$

$-1.687555$

$-1.602825$

$-4.890177$

$-3.459703$
$-2.024228$

$-1.653152$

$-2.378298-1.853887$

$-1.250002-1.614913$

$-0.208932-1.248958$

$-0.726527-1.247886$

$-3.342361-2.178887$

$-1.130340-1.704651$

$3.355220-0.585267$

$4.254753-0.333266$

$3.532845-0.436943$

$2.173234-0.764257$

$2.101770-0.836797$

$5.305843-0.104780$

$3.884284-0.293933$

$-1.260759-1.760509$

$-2.604714 \quad-2.148254$

$-3.332653-2.226258$

$-2.445404-1.904705$

$-1.200565-1.615439$

$-2.945884-2.354878$

$-4.375783-2.490689$

$1.120347-1.266797$

$2.198579-1.044804$

$3.311790-0.762289$

$2.921881-0.807223$

$1.597231-1.101845$

$2.108023-1.076187$

$4.297102-0.521479$ 


$$
0.986655
$$

$-0.582880$

3. 579409

$-3.171009$

0.194023

0.442862

$-4.611655$

$-5.338479$

$-5.308501$

$-6.689616$

$-6.663006$

$-7.356573$

1. 278430

1.730902

1.135158

2.032386

1. 424550

1.878271

5.019587

5.502992

5.955539

6.846579

7.304399

7.750105

$-0.880604$

$-1.387277$

$-0.699062$

$-1.704043$

$-1.005216$

$-1.509654$

$-4.736116$

$-7.366753$

$-8.653083$

$-7.296221$

$-4.675188$

$-1.589080$

$-2.188333$

$-1.809222$

$-0.823408$

$-0.218680$

4.668307

7.276479

9.040005

8.166422
$-2.842668-1.917183$

$3.752963-0.571384$

$1.143266-0.989473$

$-0.211655-1.591994$

$0.473314-1.235020$

$0.836453-3.566564$

$-0.521502-1.817271$

$-1.391125-0.998021$

$0.075275 \quad-2.876944$

$-1.648067-1.209465$

$-0.162146-3.103256$

$-1.031031-2.264848$

$-4.253056-2.311496$

$\begin{array}{lll}-5.191777 & -1.382172\end{array}$

$-4.688281-3.633693$

$-6.504407-1.732222$

$-5.998624-4.011731$

$-6.907688-3.057266$

$1.531468-1.039459$

$2.391090-2.034965$

$1.036949-0.126468$

$2.752770-2.107436$

$1.377590 \quad-0.179604$

$2.242296-1.177185$

$5.189574-0.295460$

$6.018989-1.302453$

$5.759573 \quad 0.968788$

$7.355337-1.067950$

$7.094560 \quad 1.225417$

$\begin{array}{ll}7.894230 & 0.202190\end{array}$

$\begin{array}{ll}-2.017345 & 0.034397\end{array}$

$-2.485659-0.406367$

$-1.274403-2.468550$

$0.427073-4.121263$

$0.908457-3.706654$

$5.530076-2.531316$

$8.123205-2.050454$

$9.174327 \quad 0.439126$

$\begin{array}{ll}7.610583 & 2.446973\end{array}$

$5.022590 \quad 1.977513$

$2.892820-2.950207$

$3.577851-3.067703$

$2.580910-1.241521$

$0.888779 \quad 0.716865$ 


$$
\begin{aligned}
& \begin{array}{lll}
5.564360 & 0.198477 & 0.848842
\end{array} \\
& \begin{array}{lll}
1.876319 & -4.839387 & -0.087185
\end{array} \\
& 2.458511-7.376174-0.810452 \\
& 2.160408-8.163875 \quad-3.409574 \\
& 1.277089-6.387965-5.281410 \\
& 0.703043-3.840846-4.570591 \\
& \begin{array}{llll}
0.135005 & -0.001766 & 0.857232
\end{array} \\
& \begin{array}{lll}
-0.132970 & 0.929010 & 2.165738
\end{array} \\
& \begin{array}{lll}
-1.899631 & 1.216794 & 2.138687
\end{array} \\
& \begin{array}{lll}
-2.782310 & 0.170880 & 1.868218
\end{array} \\
& \begin{array}{lll}
-2.372375 & 2.466719 & 2.539283
\end{array} \\
& \begin{array}{lll}
-4.150434 & 0.383598 & 2.009049
\end{array} \\
& \begin{array}{lll}
-2.397222 & -0.784779 & 1.535842
\end{array} \\
& \begin{array}{lll}
-3.746888 & 2.661726 & 2.677550
\end{array} \\
& \begin{array}{lll}
-1.666482 & 3.266994 & 2.729612
\end{array} \\
& \begin{array}{lll}
-4.652748 & 1.626184 & 2.419300
\end{array} \\
& \begin{array}{lll}
-4.837839 & -0.430750 & 1.812830
\end{array} \\
& \begin{array}{lll}
-4.119570 & 3.635248 & 2.988020
\end{array} \\
& \begin{array}{lll}
-6.143299 & 1.835849 & 2.537251
\end{array} \\
& \begin{array}{lll}
-6.383446 & 2.703145 & 3.160931
\end{array} \\
& \begin{array}{lll}
-6.594723 & 2.006719 & 1.549958
\end{array} \\
& \begin{array}{lll}
-6.632576 & 0.956231 & 2.970718
\end{array} \\
& 0.141089 \\
& 0.110083 \\
& 0.528381 \\
& 2.251394 \\
& 3.387713 \\
& 2.156465 \\
& 1.048862-0.431939 \\
& 1.019254 \\
& -2.922913-3.402503 \\
& 2.494818 \\
& -2.423587 \\
& -3.024386 \\
& 3.724052 \\
& -1.040595-2.741227 \\
& 3.848810 \\
& -0.165531-2.833047 \\
& 2.723542 \\
& -0.725830-3.186028 \\
& 1.485437 \\
& -2.076352-3.471348 \\
& 1.373256 \\
& -3.071250-2.937479 \\
& 4.585931 \\
& -0.507146-2.382166 \\
& 5.108951 \\
& 1.254676-2.543185 \\
& 2.920377 \\
& -0.116980-3.252885 \\
& 0.595172 \\
& -2.502098-3.735603 \\
& 0.412171 \\
& 1.740092-2.264833 \\
& 4.251647 \\
& 0.851453-2.177244 \\
& 5.314657 \\
& -4.226346-3.811184 \\
& 2.271664 \\
& -6.568637-3.866416 \\
& 2.429692 \\
& -7.413818-3.437025 \\
& 2.968261 \\
& -6.693427-3.709942 \\
& 1.355992 \\
& -6.510592-4.944663 \\
& 2.607814 \\
& -5.299257 \\
& -3.197493 \\
& 2.878527
\end{aligned}
$$




$\begin{array}{rrr}-5.204076 & -2.264119 & 3.635705 \\ 3.223458 & -2.174746 & 4.424693 \\ 3.886796 & -1.376359 & 3.278641 \\ 3.860915 & -1.611806 & 5.708447 \\ 3.756180 & -2.289626 & 6.560930 \\ 5.339564 & -1.414627 & 5.285429 \\ 5.982165 & -2.237186 & 5.618592 \\ 5.342985 & -1.410849 & 3.741296 \\ 6.323703 & -1.470978 & 3.036245 \\ 3.444846 & 0.109614 & 3.255756 \\ 3.858748 & 0.598235 & 2.372407 \\ 2.357196 & 0.202669 & 3.243643 \\ 3.805737 & 0.656142 & 4.133110 \\ 5.786513 & -0.490877 & 5.666768 \\ 3.392582 & -0.661033 & 5.981051 \\ 3.570881 & -2.071175 & 1.955312 \\ 2.140937 & -2.481956 & 1.862799 \\ 4.186719 & -2.981749 & 1.836335 \\ 3.827675 & -1.456835 & 1.086047 \\ 1.800453 & -2.741265 & 0.868580 \\ 1.218416 & -1.942502 & 6.307785 \\ -1.192079 & -2.287321 & 5.948081 \\ 3.587065 & -3.214973 & 4.319376\end{array}$

Structure: ${ }^{1} \mathrm{TS} 10$

$\begin{array}{lrrr}\mathrm{C} & 0.148656 & -2.377145 & -2.221724 \\ \mathrm{C} & 0.993704 & -3.542920 & -2.332843 \\ \mathrm{C} & 2.222824 & -3.189343 & -1.865976 \\ \mathrm{C} & 2.143474 & -1.796597 & -1.491995 \\ \mathrm{~N} & 0.868874 & -1.332888 & -1.690277 \\ \mathrm{H} & 0.682989 & -4.506471 & -2.710125 \\ \mathrm{H} & 3.106698 & -3.806844 & -1.794627 \\ \mathrm{C} & 2.531080 & 2.460875 & -0.954398 \\ \mathrm{C} & 3.973284 & 2.479025 & -0.877457 \\ \mathrm{C} & 4.383052 & 1.184832 & -0.802323 \\ \mathrm{C} & 3.197399 & 0.363843 & -0.898493 \\ \mathrm{~N} & 2.084381 & 1.161334 & -0.959939 \\ \mathrm{H} & 4.589654 & 3.365859 & -0.878425 \\ \mathrm{H} & 5.396113 & 0.814254 & -0.739585 \\ \mathrm{C} & -2.823246 & 0.691393 & -1.600348 \\ \mathrm{C} & -3.967833 & -0.073879 & -2.037214 \\ \mathrm{C} & -3.494421 & -1.266512 & -2.494665 \\ \mathrm{C} & -2.063455 & -1.256965 & -2.298175 \\ & & & \end{array}$


N

$\mathrm{H}$

$\mathrm{H}$

C

C

C

C

N

$\mathrm{H}$

$\mathrm{H}$

C

C

C

C

$\mathrm{Mn}$

$\mathrm{Cl}$

$\mathrm{N}$

S

O

O

C

C

C

C

$\mathrm{H}$

C

$\mathrm{H}$

C

$\mathrm{H}$

$\mathrm{H}$

C

$\mathrm{H}$

$\mathrm{H}$

$\mathrm{H}$

C

C

C

C

C

C

$\mathrm{H}$

C

C

$\mathrm{H}$

\begin{tabular}{|c|c|c|}
\hline-1.682571 & -0.051883 & -1.761731 \\
\hline-4.993129 & 0.264964 & -2.023974 \\
\hline-4.061261 & -2.079684 & -2.924579 \\
\hline-1.751313 & 2.820041 & -0.939030 \\
\hline-1.814371 & 4.240229 & -0.674684 \\
\hline-0.537084 & 4.698718 & -0.698414 \\
\hline 0.316297 & 3.553209 & -0.930705 \\
\hline-0.446764 & 2.427876 & -1.071935 \\
\hline-2.722874 & 4.805236 & -0.522967 \\
\hline-0.193968 & 5.711789 & -0.546611 \\
\hline-1.219543 & -2.352419 & -2.522412 \\
\hline 1.715798 & 3.598858 & -0.944518 \\
\hline 3.226784 & -1.026812 & -1.054532 \\
\hline-2.871246 & 2.007947 & -1.121819 \\
\hline 0.202255 & 0.552024 & -1.3423 \\
\hline 0.516574 & 1.015124 & -3.54 \\
\hline-0.230312 & 0.056092 & 0.637909 \\
\hline-0.211231 & 1.034627 & 2.087674 \\
\hline-0.4486 & 2.431018 & 1.7167 \\
\hline-1.082297 & 0.404998 & 3.085981 \\
\hline 1.469546 & 0.846833 & 2.629010 \\
\hline 2.417935 & 1.786 & 2.223440 \\
\hline 1.800489 & -0.189523 & 3.509942 \\
\hline 3.730089 & 1.652578 & 2.667852 \\
\hline 2.125101 & 2.612564 & 1.593465 \\
\hline 3.113714 & -0.306358 & \\
\hline 1.039939 & -0.877052 & 3.855231 \\
\hline 4.101387 & 0.594893 & 3.509419 \\
\hline 4.469596 & 2.379150 & 2.345069 \\
\hline 3.386836 & -1.132716 & 4.591077 \\
\hline 5.537827 & 0.381393 & 3.913673 \\
\hline 5.626420 & 0.185804 & 4.988182 \\
\hline 5.944474 & -0.495263 & 3.394226 \\
\hline 6.163506 & 1.244998 & 3.667287 \\
\hline 1.699952 & -3.522912 & 2.719601 \\
\hline 1.009870 & -2.844672 & 1.728970 \\
\hline-0.293944 & -2.387713 & 1.963091 \\
\hline-0.931109 & -2.633875 & 3.21539 \\
\hline-0.169533 & -3.288095 & 4.215970 \\
\hline 1.121390 & -3.733452 & 3.980840 \\
\hline 1.491384 & -2.666485 & 0.775418 \\
\hline-1.015453 & -1.679246 & 0.906509 \\
\hline-2.312673 & -2.266697 & 3.406742 \\
\hline-0.603004 & -3.469214 & 5.192342 \\
\hline
\end{tabular}


$1.678973-4.241148$

$-3.044254-1.752400$

$-2.424426-1.485249$

$2.930843-4.061179$

$5.118575-4.878483$

$4.940489-5.918089$

$5.136129-4.843369$

$6.070748-4.546858$

$4.006247-4.026615$

$4.026667-3.436250$

$-4.526645-1.546348$

$-4.864183-1.024057$

$-5.302225-0.620135$

$-5.457432-1.067655$

$-6.627872$

$-7.461317$

$-6.361946$

$-6.949229$

$-4.737280$

$-4.446435$

$-2.930348$

$-2.734308$

$-2.375486$

$-3.007646$

$-0.711836$

$-4.204144$

$-4.550554$

$-5.167382$

$-5.817112$

$-6.435824$

$-6.763554$

2.364808

2.203229

3.147560

2.790198

3.751763

3.569650

4.540237

5.038013

5.357797

6.310654

6.624115

7.105470

$-1.844430$
$-0.358407$

$-0.960827$

$-0.764380$

0.688248

0.300327

$-2.090694$

$-2.384694$

$-3.381566$

$-1.687349$

4.757386

2. 307971

1.102058

2. 358921

2. 651306

2. 950814

1. 559849

3.062825

3.207850

4.260413

2.483991

3.896979

1.529345

0.543064

2. 287374

1. 909559

3.752675

2. 255788

1. 383575

4.903351

4.789388

5.203798

5.434766

0.269922

$-1.118165$

$-0.092170$

$2.568402-0.761486$

$2.689396 \quad 0.587157$

$2.932438-1.704550$

$3.098516 \quad 0.989789$

$3.370252-1.325616$

3.4455840 .027658

$4.940106-0.921661$

$5.826356-1.994347$

$5.382106 \quad 0.149812$

$7.089963-2.003292$

$6.636232 \quad 0.161232$

$7.494585-0.921296$

$-1.695960-0.832024$

$\begin{array}{ll}-1.850075 & 0.463092\end{array}$

$-2.118934-1.884005$

$-2.352428 \quad 0.711326$

$-2.658791-1.659202$

$-2.768147-0.354736$

$-3.620498-2.993711$ 


\begin{tabular}{|c|c|c|c|}
\hline $\mathrm{C}$ & -1.622264 & -4.122861 & -4.278385 \\
\hline C & -2.669376 & -4.364991 & -2.144550 \\
\hline $\mathrm{C}$ & -2.199732 & -5.317024 & -4.707541 \\
\hline $\mathrm{C}$ & -3.263161 & -5.555919 & -2.552420 \\
\hline C & -3.024667 & -6.032263 & -3.841110 \\
\hline $\mathrm{F}$ & 4.283378 & -1.498014 & 1.513846 \\
\hline F & 6.767837 & -2.446879 & 1.970880 \\
\hline F & 4.934373 & -1.999764 & -3.146176 \\
\hline $\mathrm{F}$ & 7.385721 & -3.056306 & -2.682646 \\
\hline $\mathrm{F}$ & 8.321107 & -3.269559 & -0.127779 \\
\hline $\mathrm{F}$ & -2.905101 & -3.931498 & -0.894992 \\
\hline $\mathrm{F}$ & -0.831776 & -3.457542 & -5.126971 \\
\hline $\mathrm{F}$ & -1.971681 & -5.777844 & -5.941291 \\
\hline $\mathrm{F}$ & -4.049455 & -6.247052 & -1.719494 \\
\hline $\mathrm{F}$ & -3.585147 & -7.174934 & -4.243271 \\
\hline $\mathrm{F}$ & 1.464664 & 5.468668 & -3.049463 \\
\hline $\mathrm{F}$ & 3.341499 & 4.588726 & 1.219244 \\
\hline $\mathrm{F}$ & 4.495216 & 7.023280 & 1.203641 \\
\hline $\mathrm{F}$ & 2.617096 & 7.911739 & -3.042859 \\
\hline F & 4.140551 & 8.700805 & -0.920754 \\
\hline $\mathrm{F}$ & -4.892226 & 2.835560 & -3.011094 \\
\hline $\mathrm{F}$ & -7.342048 & 3.706742 & -2.248922 \\
\hline F & -7.983858 & 3.836354 & 0.399766 \\
\hline $\mathrm{F}$ & -3.667514 & 2.339437 & 1.534474 \\
\hline $\mathrm{F}$ & -6.143547 & 3.112242 & 2.290158 \\
\hline $\mathrm{H}$ & -5.018625 & -3.005912 & 4.706233 \\
\hline $\mathrm{H}$ & -4.688145 & -1.786064 & 5.926944 \\
\hline $\mathrm{C}$ & -4.224169 & 0.350989 & 4.219641 \\
\hline $\mathrm{H}$ & -4.571660 & 1.141665 & 3.548592 \\
\hline $\mathrm{H}$ & -4.498253 & 0.638537 & 5.240540 \\
\hline $\mathrm{H}$ & -3.136106 & 0.320227 & 4.134950 \\
\hline O & -7.198373 & -0.860598 & 4.623415 \\
\hline $\mathrm{H}$ & -4.995955 & -2.542995 & 2.410523 \\
\hline
\end{tabular}

\section{Structure: ${ }^{3}$ TS10}

$\begin{array}{llll}\mathrm{C} & 0.345152 & -2.398441 & -2.099589 \\ \mathrm{C} & 1.259695 & -3.513553 & -2.116636 \\ \mathrm{C} & 2.418145 & -3.090810 & -1.539518 \\ \mathrm{C} & 2.235610 & -1.702381 & -1.195516 \\ \mathrm{~N} & 0.961195 & -1.312640 & -1.523876 \\ \mathrm{H} & 1.033595 & -4.496360 & -2.503680 \\ \mathrm{H} & 3.323710 & -3.657014 & -1.376138 \\ \mathrm{C} & 2.355946 & 2.572650 & -0.899103\end{array}$




\begin{tabular}{|c|c|c|c|}
\hline C & 3.786612 & 2.675430 & -0.732211 \\
\hline C & 4.254529 & 1.417783 & -0.519351 \\
\hline C & 3.124549 & 0.523827 & -0.626047 \\
\hline $\mathrm{N}$ & 1.982635 & 1.253142 & -0.825783 \\
\hline $\mathrm{H}$ & 4.351911 & 3.594999 & -0.758792 \\
\hline $\mathrm{H}$ & 5.275905 & 1.108486 & -0.352436 \\
\hline C & -2.843744 & 0.460643 & -1.615103 \\
\hline C & -3.911014 & -0.390115 & -2.082940 \\
\hline $\mathrm{C}$ & -3.336721 & -1.537889 & -2.535891 \\
\hline C & -1.917255 & -1.425872 & -2.301577 \\
\hline $\mathrm{N}$ & -1.646116 & -0.198338 & -1.747514 \\
\hline $\mathrm{H}$ & -4.958278 & -0.129765 & -2.095538 \\
\hline $\mathrm{H}$ & -3.828143 & -2.390385 & -2.981431 \\
\hline $\mathrm{C}$ & -1.932671 & 2.661632 & -1.001437 \\
\hline C & -2.078109 & 4.087910 & -0.833317 \\
\hline C & -0.831381 & 4.620457 & -0.895957 \\
\hline C & 0.092114 & 3.520987 & -1.047359 \\
\hline $\mathrm{N}$ & -0.606195 & 2.345854 & -1.115355 \\
\hline $\mathrm{H}$ & -3.018994 & 4.606729 & -0.721954 \\
\hline $\mathrm{H}$ & -0.549232 & 5.659619 & -0.813484 \\
\hline $\mathrm{C}$ & -0.997074 & -2.461952 & -2.487004 \\
\hline $\mathrm{C}$ & 1.481965 & 3.654889 & -1.018620 \\
\hline $\mathrm{C}$ & 3.240614 & -0.866099 & -0.706370 \\
\hline $\mathrm{C}$ & -2.998898 & 1.779913 & -1.174897 \\
\hline Mn & 0.151163 & 0.507075 & -1.189877 \\
\hline $\mathrm{Cl}$ & 0.581027 & 0.938117 & -3.461625 \\
\hline $\mathrm{N}$ & -0.162339 & 0.124043 & 0.621111 \\
\hline S & -0.181853 & 1.113147 & 1.974723 \\
\hline O & -0.305543 & 2.526656 & 1.585025 \\
\hline O & -1.158054 & 0.567320 & 2.933550 \\
\hline C & 1.429231 & 0.965763 & 2.759009 \\
\hline C & 2.445127 & 1.851177 & 2.403700 \\
\hline $\mathrm{C}$ & 1.632917 & 0.015464 & 3.760562 \\
\hline C & 3.694177 & 1.734754 & 3.010690 \\
\hline $\mathrm{H}$ & 2.251674 & 2.633836 & 1.684619 \\
\hline C & 2.883993 & -0.088012 & 4.360345 \\
\hline $\mathrm{H}$ & 0.817778 & -0.621697 & 4.074557 \\
\hline C & 3.940482 & 0.750988 & 3.977007 \\
\hline $\mathrm{H}$ & 4.486121 & 2.421591 & 2.721697 \\
\hline $\mathrm{H}$ & 3.054316 & -0.857916 & 5.106085 \\
\hline C & 5.318955 & 0.547818 & 4.555387 \\
\hline $\mathrm{H}$ & 5.277188 & 0.308515 & 5.623432 \\
\hline $\mathrm{H}$ & 5.812765 & -0.295915 & 4.056866 \\
\hline $\mathrm{H}$ & 5.950072 & 1.433284 & 4.424740 \\
\hline
\end{tabular}




\begin{tabular}{|c|c|c|c|}
\hline$C$ & 1.763640 & -3.335899 & 3.055252 \\
\hline C & 1.104690 & -2.727537 & 2.000512 \\
\hline $\mathrm{C}$ & -0.222390 & -2.300135 & 2.143410 \\
\hline C & -0.904165 & -2.496173 & 3.381496 \\
\hline C & -0.176119 & -3.078982 & 4.449221 \\
\hline C & 1.132625 & -3.502804 & 4.297964 \\
\hline $\mathrm{H}$ & 1.628442 & -2.578764 & 1.065542 \\
\hline C & -0.923668 & -1.691849 & 1.018885 \\
\hline$C$ & -2.298782 & -2.153156 & 3.497471 \\
\hline $\mathrm{H}$ & -0.650165 & -3.220658 & 5.412991 \\
\hline $\mathrm{H}$ & 1.664564 & -3.956241 & 5.123998 \\
\hline C & -3.001222 & -1.726939 & 2.349131 \\
\hline C & -2.335581 & -1.523183 & 1.151064 \\
\hline O & 3.022751 & -3.856662 & 2.778393 \\
\hline C & 5.199106 & -4.639003 & 3.214411 \\
\hline $\mathrm{H}$ & 5.002831 & -5.676815 & 3.508784 \\
\hline $\mathrm{H}$ & 5.292265 & -4.613460 & 2.126386 \\
\hline $\mathrm{H}$ & 6.119830 & -4.300775 & 3.687558 \\
\hline C & 4.048862 & -3.785432 & 3.686473 \\
\hline O & 4.004801 & -3.167662 & 4.721412 \\
\hline C & -4.493263 & -1.542375 & 2.463446 \\
\hline $\mathrm{C}$ & -4.889988 & -0.944919 & 3.831103 \\
\hline $\mathrm{C}$ & -5.253334 & -0.689292 & 1.432445 \\
\hline $\mathrm{H}$ & -5.364478 & -1.193611 & 0.467742 \\
\hline C & -6.610761 & -0.417659 & 2.128428 \\
\hline $\mathrm{H}$ & -7.414254 & -1.065030 & 1.760010 \\
\hline C & -6.386372 & -0.729230 & 3.623858 \\
\hline $\mathrm{H}$ & -6.960560 & 0.615020 & 2.024614 \\
\hline $\mathrm{H}$ & -4.701194 & 0.233120 & 1.255949 \\
\hline $\mathrm{C}$ & -4.485859 & -1.941448 & 4.911182 \\
\hline C & -2.962274 & -2.206077 & 4.862683 \\
\hline $\mathrm{H}$ & -2.758348 & -3.171642 & 5.341688 \\
\hline $\mathrm{H}$ & -2.442937 & -1.458979 & 5.480984 \\
\hline $\mathrm{H}$ & -2.896358 & -1.223235 & 0.278223 \\
\hline $\mathrm{H}$ & -0.565269 & -1.991074 & 0.049511 \\
\hline $\mathrm{C}$ & -4.370468 & 2.290223 & -0.902555 \\
\hline $\mathrm{C}$ & -4.749066 & 2.570976 & 0.413991 \\
\hline $\mathrm{C}$ & -5.331724 & 2.483529 & -1.897956 \\
\hline C & -6.042675 & 2.961293 & 0.742491 \\
\hline $\mathrm{C}$ & -6.628570 & 2.895916 & -1.595986 \\
\hline C & -6.986791 & 3.128351 & -0.268725 \\
\hline C & 2.046646 & 5.032258 & -1.048657 \\
\hline 0 & 1.895575 & 5.839732 & -2.182446 \\
\hline & 2.724707 & 5.585917 & 0.042317 \\
\hline
\end{tabular}




\begin{tabular}{|c|c|c|c|}
\hline$C$ & 2.398405 & 7.137991 & -2.233930 \\
\hline C & 3.243409 & 6.877837 & 0.011085 \\
\hline C & 3.076595 & 7.656888 & -1.132457 \\
\hline $\mathrm{C}$ & 4.572377 & -1.453238 & -0.384120 \\
\hline $\mathrm{C}$ & 4.981444 & -1.590662 & 0.943598 \\
\hline C & 5.484648 & -1.816212 & -1.379135 \\
\hline $\mathrm{C}$ & 6.259423 & -2.028801 & 1.276687 \\
\hline C & 6.760280 & -2.285564 & -1.068779 \\
\hline C & 7.150313 & -2.385441 & 0.266559 \\
\hline $\mathrm{C}$ & -1.507742 & -3.767384 & -2.990613 \\
\hline $\mathrm{C}$ & -1.173285 & -4.251930 & -4.257607 \\
\hline C & -2.323524 & -4.569237 & -2.185985 \\
\hline C & -1.636528 & -5.485253 & -4.713537 \\
\hline C & -2.804504 & -5.800345 & -2.622286 \\
\hline C & -2.456271 & -6.258203 & -3.892761 \\
\hline $\mathrm{F}$ & 4.134271 & -1.294264 & 1.934798 \\
\hline $\mathrm{F}$ & 6.631916 & -2.121274 & 2.562873 \\
\hline $\mathrm{F}$ & 5.143122 & -1.707826 & -2.667180 \\
\hline $\mathrm{F}$ & 7.614548 & -2.627228 & -2.037677 \\
\hline $\mathrm{F}$ & 8.371837 & -2.823574 & 0.576045 \\
\hline $\mathrm{F}$ & -2.659854 & -4.151806 & -0.954991 \\
\hline F & -0.382802 & -3.531854 & -5.059714 \\
\hline $\mathrm{F}$ & -1.303670 & -5.929295 & -5.929016 \\
\hline $\mathrm{F}$ & -3.585319 & -6.546606 & -1.833824 \\
\hline $\mathrm{F}$ & -2.906813 & -7.438674 & -4.321509 \\
\hline $\mathrm{F}$ & 1.247453 & 5.370510 & -3.253375 \\
\hline $\mathrm{F}$ & 2.897728 & 4.869451 & 1.163953 \\
\hline $\mathrm{F}$ & 3.890149 & 7.375898 & 1.069921 \\
\hline $\mathrm{F}$ & 2.238938 & 7.885033 & -3.330412 \\
\hline $\mathrm{F}$ & 3.566051 & 8.897493 & -1.171807 \\
\hline $\mathrm{F}$ & -5.024326 & 2.247927 & -3.180139 \\
\hline $\mathrm{F}$ & -7.530418 & 3.063883 & -2.567929 \\
\hline $\mathrm{F}$ & -8.232541 & 3.496901 & 0.032807 \\
\hline $\mathrm{F}$ & -3.866921 & 2.401191 & 1.408046 \\
\hline F & -6.394045 & 3.125655 & 2.024329 \\
\hline $\mathrm{H}$ & -5.031840 & -2.879569 & 4.750374 \\
\hline $\mathrm{H}$ & -4.767601 & -1.583138 & 5.906743 \\
\hline C & -4.286928 & 0.460094 & 4.090269 \\
\hline $\mathrm{H}$ & -4.657624 & 1.208065 & 3.384130 \\
\hline H & -4.569698 & 0.787615 & 5.096625 \\
\hline $\mathrm{H}$ & -3.198162 & 0.458646 & 4.002130 \\
\hline O & -7.251136 & -0.793257 & 4.469525 \\
\hline & -4.942509 & -2.550478 & 2.432957 \\
\hline
\end{tabular}


Structure: ${ }^{5}$ TS10

C

C

C

C

$\mathrm{N}$

$\mathrm{H}$

$\mathrm{H}$

C

C

C

C

$\mathrm{N}$

$\mathrm{H}$

$\mathrm{H}$

C

C

C

C

$\mathrm{N}$

$\mathrm{H}$

$\mathrm{H}$

C

C

C

C

$\mathrm{N}$

$\mathrm{H}$

$\mathrm{H}$

C

C

C

C

$\mathrm{Mn}$

$\mathrm{Cl}$

$\mathrm{N}$

S

O

0

C

C

C

C
0.414955

1.350047

2.503218

2. 291886

1.012108

1.140916

3.424560

2. 320266

3. 748877

4.249322

3. 142004

1.977184

4. 292417

5.278934

$-2.831926$

$-3.879515$

$-3.279507$

$-1.864721$

$-1.623411$

$-4.931185$

$-3.749920$

$-1.967825$

$-2.145868$

$-0.910517$

0.039286

$-0.630336$

$-3.098618$

$-0.653582$

$-0.923614$

1. 424353

3.286095

$-3.015211$

0.172612

0.573860

$-0.198199$

$-0.115323$

$-0.188630$

$-1.089975$

1.505125

2.545631

1. 700507

3. 807175
$-2.391553$

$-3.491283$

$-2.032422$

$-3.030553-1.461496$

$-1.637141-1.152824$

$-1.281640-1.488004$

$-4.487643-2.382199$

$-3.569590-1.292792$

$2.653615-0.913132$

$2.787202-0.765424$

$1.539950-0.563317$

$0.620037-0.654466$

$1.324623-0.841175$

$3.719691-0.797609$

$1.256616-0.402395$

$0.420919-1.648473$

$-0.459879-2.108669$

$-1.607988-2.525117$

$-1.463568-2.277013$

$-0.218713-1.750174$

$-0.219736-2.139460$

$-2.480820-2.953821$

$2.647496-1.047852$

$4.070073-0.878483$

$4.630234-0.914161$

$3.553560-1.057251$

$2.362524-1.141269$

$4.569855-0.783754$

$5.675047-0.821922$

$-2.486643-2.428779$

$3.718311-1.018479$

$-0.765982-0.703801$

$1.745730-1.233164$

$0.546537-1.205956$

$0.900667-3.505348$

$\begin{array}{ll}0.114561 & 0.635387\end{array}$

$1.111056 \quad 1.972651$

$2.529283 \quad 1.578810$

$0.612620 \quad 2.962391$

$0.896802 \quad 2.724586$

$1.754132 \quad 2.373114$

$\begin{array}{ll}-0.097049 & 3.685732\end{array}$

$1.563694 \quad 2.937238$ 


\begin{tabular}{|c|c|c|c|}
\hline $\mathrm{H}$ & 2.364096 & 2.571448 & 1.689448 \\
\hline C & 2.960987 & -0.271744 & 4.245977 \\
\hline $\mathrm{H}$ & 0.869711 & -0.711951 & 4.002507 \\
\hline C & 4.040602 & 0.536733 & 3.859980 \\
\hline $\mathrm{H}$ & 4.618992 & 2.227821 & 2.649929 \\
\hline $\mathrm{H}$ & 3.119790 & -1.074509 & 4.959179 \\
\hline C & 5.420816 & 0.263331 & 4.405302 \\
\hline $\mathrm{H}$ & 5.404966 & 0.139709 & 5.494109 \\
\hline $\mathrm{H}$ & 5.815919 & -0.669835 & 3.985253 \\
\hline $\mathrm{H}$ & 6.121194 & 1.069608 & 4.163178 \\
\hline C & 1.640778 & -3.503211 & 3.039251 \\
\hline $\mathrm{C}$ & 0.989554 & -2.885216 & 1.985368 \\
\hline C & -0.303717 & -2.368962 & 2.155716 \\
\hline C & -0.957664 & -2.485947 & 3.419901 \\
\hline C & -0.230844 & -3.074672 & 4.485563 \\
\hline $\mathrm{C}$ & 1.042632 & -3.586153 & 4.307092 \\
\hline $\mathrm{H}$ & 1.488511 & -2.809304 & 1.027569 \\
\hline C & -1.002709 & -1.758542 & 1.041733 \\
\hline C & -2.327955 & -2.068832 & 3.557092 \\
\hline $\mathrm{H}$ & -0.680621 & -3.154582 & 5.467912 \\
\hline $\mathrm{H}$ & 1.572740 & -4.047844 & 5.129783 \\
\hline C & -3.042805 & -1.664073 & 2.405676 \\
\hline C & -2.396688 & -1.526108 & 1.188395 \\
\hline O & 2.851116 & -4.117654 & 2.741897 \\
\hline $\mathrm{C}$ & 5.017252 & -4.958241 & 3.084516 \\
\hline $\mathrm{H}$ & 4.725030 & -6.001675 & 3.249384 \\
\hline $\mathrm{H}$ & 5.158435 & -4.821355 & 2.009432 \\
\hline $\mathrm{H}$ & 5.941839 & -4.746880 & 3.619432 \\
\hline $\mathrm{C}$ & 3.920711 & -4.060318 & 3.598999 \\
\hline O & 3.947101 & -3.415897 & 4.618179 \\
\hline $\mathrm{C}$ & -4.526193 & -1.438465 & 2.543446 \\
\hline C & -4.882593 & -0.771186 & 3.889216 \\
\hline C & -5.293904 & -0.623136 & 1.488852 \\
\hline $\mathrm{H}$ & -5.431002 & -1.169631 & 0.550536 \\
\hline $\mathrm{C}$ & -6.633412 & -0.302287 & 2.198464 \\
\hline $\mathrm{H}$ & -7.448576 & -0.962830 & 1.882487 \\
\hline C & -6.379212 & -0.537438 & 3.703221 \\
\hline $\mathrm{H}$ & -6.979413 & 0.725485 & 2.047428 \\
\hline $\mathrm{H}$ & -4.734375 & 0.282958 & 1.259349 \\
\hline C & -4.473812 & -1.724514 & 5.006173 \\
\hline C & -2.957699 & -2.023094 & 4.938168 \\
\hline $\mathrm{H}$ & -2.761589 & -2.959838 & 5.473568 \\
\hline $\mathrm{H}$ & -2.407830 & -1.248742 & 5.493528 \\
\hline $\mathrm{H}$ & -2.959305 & -1.220795 & 0.317776 \\
\hline
\end{tabular}


C

C

C

C

C

C

C

C

C

C

C

C

C

C

C

C

C

F

F

F

F

F

F

F

F

F

F

F

F

F

F

F

F

F

F

F
$-0.642230$

$-4.398935$

$-4.800458$

$-5.348162$

$-6.101429$

$-6.653774$

$-7.033351$

1.960445

1.801092

2. 619144

2. 277276

3. 111172

2.936464

4.633994

4.958590

5.633675

6.236496

6.915672

7. 217760

$-1.407324$

$-1.069738$

$-2.201662$

$-1.510201$

$-2.659015$

$-2.308893$

4.031355

6.527916

5.374174

7.856072

8.442832

$-2.538838$

$-0.299678$

$-1.175676$

$-3.419162$

$-2.737268$

1.169838

2.799223

3. 740221

2. 110762

3. 400799

$-5.019774$

$-7.543049$

$-8.286397$

$-3.929091$
$-2.019616$

2. 242012

0.063297

2. 572680

$-0.998433$

2.385370

0.299714

2.965080

$-2.013983$

2.794367

0.593900

3.078436

$-1.746903$

5.106762

$-0.435819$

$-1.013714$

$5.941204-2.126259$

5.643010

0.097952

7.250380

$-2.138357$

6.945728

0.105745

7.752206

$-1.017566$

$-1.324022$

$-0.390913$

$-1.719109$

0.907235

$-1.417857$

$-1.362907$

$\begin{array}{ll}-2.157586 & 1.241958\end{array}$

$-1.871414$

$-1.054935$

$\begin{array}{ll}-2.238464 & 0.256155\end{array}$

$-3.813095$

$-2.903808$

$-4.314082-4.163370$

$-4.615873-2.079079$
-5.564986

$-5.564986$

$-4.593370$

$-5.864937-2.489019$

$-6.339040-3.753079$

$-1.670572$

1.869948

$-2.498228$

2.507046

$-1.048605$

$-2.620905$

$-1.949160$

$-2.000724$

$-2.666207$

0.565877

$-4.181759$

$-0.854040$

$-3.592543$

$-4.983623$

$-6.024715-5.802531$

$-6.612537-1.681947$

$-7.536430-4.156846$

$5.487917-3.214417$

$4.898419 \quad 1.199066$

$7.428219 \quad 1.182177$

$8.023871-3.215369$

$9.003035-1.019281$

$2.106595-3.282143$

$2.912368-2.737495$

$3.447319-0.167181$

$2.458925 \quad 1.311773$ 


$\begin{array}{lrrr}\mathrm{F} & -6.470579 & 3.184838 & 1.862390 \\ \mathrm{H} & -5.041459 & -2.657900 & 4.902006 \\ \mathrm{H} & -4.725899 & -1.313967 & 5.989474 \\ \mathrm{C} & -4.248178 & 0.632287 & 4.071008 \\ \mathrm{H} & -4.625437 & 1.355739 & 3.343073 \\ \mathrm{H} & -4.499087 & 1.007629 & 5.069007 \\ \mathrm{H} & -3.161881 & 0.607262 & 3.956000 \\ \mathrm{O} & -7.225117 & -0.538663 & 4.570126 \\ \mathrm{H} & -4.994732 & -2.438035 & 2.570844\end{array}$

\section{Structure: ${ }^{3}$ INT9}

\begin{tabular}{|c|c|c|c|}
\hline C & -0.022047 & -2.288434 & -2.094094 \\
\hline C & 0.693512 & -3.543416 & -2.170968 \\
\hline $\mathrm{C}$ & 1.980244 & -3.284920 & -1.81558 \\
\hline $\mathrm{C}$ & 2.056692 & -1.872412 & -1.5175 \\
\hline $\mathrm{N}$ & 0.823366 & -1.295096 & -1.67132 \\
\hline $\mathrm{H}$ & 0.266456 & -4.492364 & -2.46193 \\
\hline $\mathrm{H}$ & 2.803413 & -3.982130 & -1.7531 \\
\hline $\mathrm{C}$ & 2.843450 & 2.309931 & -0.7449 \\
\hline C & 4.277634 & 2.189529 & -0.6025 \\
\hline C & 4.571409 & 0.864925 & -0.6755 \\
\hline C & 3.320599 & 0.168705 & -0.8867 \\
\hline $\mathrm{N}$ & 2.286241 & 1.067155 & -0.9028 \\
\hline $\mathrm{H}$ & 4.966452 & 3.012498 & -0.4797 \\
\hline $\mathrm{H}$ & 5.547116 & 0.403400 & -0.6290 \\
\hline C & -2.640865 & 1.137726 & -1.7435 \\
\hline C & -3.866288 & 0.463878 & -2.1125 \\
\hline C & -3.528384 & -0.809981 & -2.4540 \\
\hline C & -2.100638 & -0.930173 & -2.2677 \\
\hline $\mathrm{N}$ & -1.584686 & 0.271176 & -1.8485 \\
\hline $\mathrm{H}$ & -4.849063 & 0.911650 & -2.1366 \\
\hline $\mathrm{H}$ & -4.182010 & -1.595376 & -2.8059 \\
\hline C & -1.360826 & 3.149505 & -1.06706 \\
\hline $\mathrm{C}$ & -1.288455 & 4.565887 & -0.7758 \\
\hline 0 & 0.027624 & 4.873522 & -0.6661 \\
\hline$C$ & 0.762353 & 3.640635 & -0.85679 \\
\hline $\mathrm{N}$ & -0.104774 & 2.614022 & -1.1079 \\
\hline F & -2.136755 & 5.230284 & -0.6928 \\
\hline & 0.466245 & 5.836538 & -0.4498 \\
\hline$C$ & -1.389113 & -2.135031 & -2.36685 \\
\hline 0 & 2.147301 & 3.523319 & -0.7136 \\
\hline & 3.224466 & -1.208193 & -1.13008 \\
\hline & -2.555978 & 2.467453 & -1.310 \\
\hline
\end{tabular}




\begin{tabular}{|c|c|c|c|}
\hline $\mathrm{Mn}$ & 0.357333 & 0.671276 & -1.357718 \\
\hline $\mathrm{Cl}$ & 0.817900 & 1.079052 & -3.553366 \\
\hline $\mathrm{N}$ & -0.108373 & 0.185559 & 0.614385 \\
\hline S & 0.031580 & 1.096353 & 1.996802 \\
\hline O & 0.293390 & 2.503727 & 1.691909 \\
\hline O & -1.148423 & 0.745729 & 2.854347 \\
\hline C & 1.474194 & 0.479759 & 2.870000 \\
\hline C & 2.732527 & 0.956296 & 2.495037 \\
\hline C & 1.343985 & -0.525548 & 3.826266 \\
\hline $\mathrm{C}$ & 3.866703 & 0.369904 & 3.044672 \\
\hline $\mathrm{H}$ & 2.816748 & 1.769995 & 1.787735 \\
\hline $\mathrm{C}$ & 2.492869 & -1.106765 & 4.363735 \\
\hline $\mathrm{H}$ & 0.357395 & -0.863855 & 4.121588 \\
\hline $\mathrm{C}$ & 3.767437 & -0.689389 & 3.960262 \\
\hline $\mathrm{H}$ & 4.846549 & 0.719789 & 2.730081 \\
\hline $\mathrm{H}$ & 2.395512 & -1.925508 & 5.069989 \\
\hline $\mathrm{C}$ & 5.012305 & -1.369585 & 4.479192 \\
\hline $\mathrm{H}$ & 4.771422 & -2.321532 & 4.963126 \\
\hline $\mathrm{H}$ & 5.713496 & -1.568154 & 3.659953 \\
\hline $\mathrm{H}$ & 5.536693 & -0.743429 & 5.212348 \\
\hline C & 1.200836 & -3.654886 & 2.164633 \\
\hline $\mathrm{C}$ & 0.776098 & -2.507135 & 1.511377 \\
\hline C & -0.556164 & -2.115510 & 1.602392 \\
\hline $\mathrm{C}$ & -1.487150 & -2.890572 & 2.350405 \\
\hline C & -1.015195 & -4.069017 & 2.985535 \\
\hline $\mathrm{C}$ & 0.306694 & -4.453408 & 2.897237 \\
\hline $\mathrm{H}$ & 1.488952 & -1.890012 & 0.981926 \\
\hline C & -1.037753 & -0.907869 & 0.839612 \\
\hline C & -2.859740 & -2.461708 & 2.474085 \\
\hline $\mathrm{H}$ & -1.694987 & -4.696080 & 3.549090 \\
\hline $\mathrm{H}$ & 0.658529 & -5.348327 & 3.393664 \\
\hline $\mathrm{C}$ & -3.301197 & -1.270752 & 1.871749 \\
\hline $\mathrm{C}$ & -2.387428 & -0.462717 & 1.218294 \\
\hline O & 2.526788 & -4.011730 & 2.012700 \\
\hline $\mathrm{C}$ & 4.645747 & -4.816868 & 2.692621 \\
\hline $\mathrm{H}$ & 5.063781 & -5.474568 & 3.456211 \\
\hline $\mathrm{H}$ & 4.626245 & -5.313630 & 1.719757 \\
\hline $\mathrm{H}$ & 5.279607 & -3.931619 & 2.609256 \\
\hline $\mathrm{C}$ & 3.270611 & -4.386627 & 3.114683 \\
\hline O & 2.857713 & -4.336997 & 4.246455 \\
\hline $\mathrm{C}$ & -4.750364 & -0.887953 & 1.997942 \\
\hline $\mathrm{C}$ & -5.303297 & -1.243730 & 3.389311 \\
\hline C & -5.206409 & 0.558559 & 1.747721 \\
\hline $\mathrm{H}$ & -5.183739 & 0.815860 & 0.684979 \\
\hline
\end{tabular}




\begin{tabular}{|c|c|c|c|}
\hline C & -6.636741 & 0.570918 & 2.334770 \\
\hline $\mathrm{H}$ & -7.400326 & 0.391969 & 1.567714 \\
\hline $\mathrm{C}$ & -6.695496 & -0.606547 & 3.328849 \\
\hline $\mathrm{H}$ & -6.900141 & 1.513164 & 2.826175 \\
\hline $\mathrm{H}$ & -4.559077 & 1.266612 & 2.269699 \\
\hline C & -5.252046 & -2.761754 & 3.526747 \\
\hline C & -3.800249 & -3.277432 & 3.347159 \\
\hline $\mathrm{H}$ & -3.822370 & -4.307660 & 2.969463 \\
\hline $\mathrm{H}$ & -3.317492 & -3.340970 & 4.331297 \\
\hline $\mathrm{H}$ & -2.700770 & 0.484937 & 0.799990 \\
\hline $\mathrm{H}$ & -1.247772 & -1.345530 & -0.153557 \\
\hline C & -3.830200 & 3.171004 & -0.990669 \\
\hline $\mathrm{C}$ & -4.168620 & 3.380860 & 0.349706 \\
\hline C & -4.753383 & 3.582046 & -1.954162 \\
\hline C & -5.394242 & 3.916669 & 0.729523 \\
\hline $\mathrm{C}$ & -5.978085 & 4.147985 & -1.600558 \\
\hline C & -6.300852 & 4.309685 & -0.253735 \\
\hline C & 2.926981 & 4.768175 & -0.465495 \\
\hline C & 3.037589 & 5.760980 & -1.445127 \\
\hline $\mathrm{C}$ & 3.567154 & 5.002913 & 0.755781 \\
\hline C & 3.751248 & 6.937065 & -1.224840 \\
\hline $\mathrm{C}$ & 4.293849 & 6.166400 & 0.995981 \\
\hline $\mathrm{C}$ & 4.383404 & 7.137843 & 0.000729 \\
\hline $\mathrm{C}$ & 4.491351 & -1.992464 & -1.061924 \\
\hline C & 5.132540 & -2.238532 & 0.153759 \\
\hline $\mathrm{C}$ & 5.132447 & -2.445225 & -2.220158 \\
\hline C & 6.376567 & -2.857328 & 0.222208 \\
\hline C & 6.362521 & -3.100600 & -2.174398 \\
\hline $\mathrm{C}$ & 6.990812 & -3.301766 & -0.946366 \\
\hline $\mathrm{C}$ & -2.177756 & -3.371038 & -2.638494 \\
\hline C & -2.109226 & -4.057783 & -3.852785 \\
\hline C & -3.015563 & -3.900560 & -1.652891 \\
\hline C & -2.843080 & -5.222410 & -4.078947 \\
\hline C & -3.762098 & -5.056333 & -1.853447 \\
\hline $\mathrm{C}$ & -3.673396 & -5.720559 & -3.076200 \\
\hline F & 4.553542 & -1.861617 & 1.305705 \\
\hline $\mathrm{F}$ & 6.980485 & -3.040480 & 1.409666 \\
\hline $\mathrm{F}$ & 4.570610 & -2.243139 & -3.415064 \\
\hline F & 6.950681 & -3.521761 & -3.297945 \\
\hline $\mathrm{F}$ & 8.175468 & -3.914023 & -0.889579 \\
\hline $\mathrm{F}$ & -3.115917 & -3.284327 & -0.457155 \\
\hline $\mathrm{F}$ & -1.317465 & -3.606481 & -4.829927 \\
\hline F & -2.759720 & -5.861109 & -5.249590 \\
\hline & -4.553303 & -5.534207 & -0.883841 \\
\hline
\end{tabular}




$\begin{array}{rrr}-4.380987 & -6.833190 & -3.283974 \\ 2.441087 & 5.596773 & -2.631270 \\ 3.500880 & 4.091913 & 1.738293 \\ 4.898815 & 6.359802 & 2.173583 \\ 3.838963 & 7.868677 & -2.180233 \\ 5.075044 & 8.258753 & 0.221070 \\ -4.486889 & 3.404849 & -3.253639 \\ -6.848469 & 4.526775 & -2.542091 \\ -7.481552 & 4.827178 & 0.093347 \\ -3.317647 & 2.995566 & 1.314307 \\ -5.719446 & 4.018818 & 2.025427 \\ -5.902171 & -3.209270 & 2.765816 \\ -5.636849 & -3.088799 & 4.498001 \\ -4.547571 & -0.533231 & 4.539864 \\ -4.615589 & 0.556539 & 4.465052 \\ -4.983025 & -0.831857 & 5.498867 \\ -3.481487 & -0.781830 & 4.541927 \\ -7.670881 & -0.971326 & 3.945875 \\ -5.297751 & -1.517509 & 1.275289\end{array}$

\section{Structure: ${ }^{5}$ INT9}

$\begin{array}{lrrr}\mathrm{C} & 0.089012 & -2.296787 & -2.154406 \\ \mathrm{C} & 0.800335 & -3.552783 & -2.134246 \\ \mathrm{C} & 2.072105 & -3.284118 & -1.734962 \\ \mathrm{C} & 2.152106 & -1.861972 & -1.507417 \\ \mathrm{~N} & 0.929354 & -1.285936 & -1.753461 \\ \mathrm{H} & 0.376217 & -4.513721 & -2.386486 \\ \mathrm{H} & 2.883672 & -3.982649 & -1.592622 \\ \mathrm{C} & 2.909193 & 2.328618 & -0.747133 \\ \mathrm{C} & 4.330409 & 2.206640 & -0.520774 \\ \mathrm{C} & 4.631270 & 0.883030 & -0.585094 \\ \mathrm{C} & 3.399776 & 0.183515 & -0.872145 \\ \mathrm{~N} & 2.369118 & 1.083998 & -0.952322 \\ \mathrm{H} & 5.007811 & 3.029574 & -0.346834 \\ \mathrm{H} & 5.602919 & 0.422986 & -0.480471 \\ \mathrm{C} & -2.545230 & 1.108628 & -1.914126 \\ \mathrm{C} & -3.760444 & 0.420366 & -2.282743 \\ \mathrm{C} & -3.410324 & -0.851257 & -2.620451 \\ \mathrm{C} & -1.983906 & -0.960514 & -2.429922 \\ \mathrm{~N} & -1.480910 & 0.252725 & -2.027647 \\ \mathrm{H} & -4.748016 & 0.857581 & -2.299912 \\ \mathrm{H} & -4.056718 & -1.647610 & -2.960299 \\ \mathrm{C} & -1.295657 & 3.128254 & -1.205016\end{array}$




\begin{tabular}{|c|c|c|c|}
\hline $\mathrm{C}$ & -1.244898 & 4.529224 & -0.855140 \\
\hline C & 0.063458 & 4.849276 & -0.696867 \\
\hline $\mathrm{C}$ & 0.823120 & 3.640132 & -0.919189 \\
\hline $\mathrm{N}$ & -0.025365 & 2.618175 & -1.249644 \\
\hline $\mathrm{H}$ & -2.104285 & 5.176625 & -0.757696 \\
\hline $\mathrm{H}$ & 0.481727 & 5.805301 & -0.419392 \\
\hline $\mathrm{C}$ & -1.266070 & -2.161854 & -2.479707 \\
\hline $\mathrm{C}$ & 2.202984 & 3.535364 & -0.723598 \\
\hline $\mathrm{C}$ & 3.309474 & -1.195364 & -1.094359 \\
\hline $\mathrm{C}$ & -2.477777 & 2.437575 & -1.477926 \\
\hline $\mathrm{Mn}$ & 0.473719 & 0.695601 & -1.586702 \\
\hline $\mathrm{Cl}$ & 1.059013 & 1.105647 & -3.905250 \\
\hline $\mathrm{N}$ & -0.249698 & 0.212692 & 0.682181 \\
\hline S & -0.192015 & 1.124650 & 2.023863 \\
\hline O & 0.082059 & 2.532059 & 1.703828 \\
\hline O & -1.419744 & 0.800431 & 2.852021 \\
\hline $\mathrm{C}$ & 1.211916 & 0.528775 & 2.974291 \\
\hline $\mathrm{C}$ & 2.483920 & 0.977470 & 2.609362 \\
\hline $\mathrm{C}$ & 1.054278 & -0.487967 & 3.914311 \\
\hline $\mathrm{C}$ & 3.601791 & 0.365096 & 3.165171 \\
\hline $\mathrm{H}$ & 2.588815 & 1.788336 & 1.899769 \\
\hline $\mathrm{C}$ & 2.186610 & -1.097572 & 4.456027 \\
\hline $\mathrm{H}$ & 0.057838 & -0.815479 & 4.189159 \\
\hline C & 3.473487 & -0.698557 & 4.072250 \\
\hline $\mathrm{H}$ & 4.591669 & 0.696347 & 2.862022 \\
\hline $\mathrm{H}$ & 2.068023 & -1.924448 & 5.149823 \\
\hline C & 4.698711 & -1.398524 & 4.612540 \\
\hline $\mathrm{H}$ & 4.447252 & -2.393797 & 4.992860 \\
\hline $\mathrm{H}$ & 5.459714 & -1.509853 & 3.831599 \\
\hline $\mathrm{H}$ & 5.154751 & -0.832010 & 5.434897 \\
\hline $\mathrm{C}$ & 1.052405 & -3.647185 & 2.108613 \\
\hline $\mathrm{C}$ & 0.619756 & -2.490100 & 1.479072 \\
\hline $\mathrm{C}$ & -0.713713 & -2.106828 & 1.584968 \\
\hline $\mathrm{C}$ & -1.633956 & -2.888476 & 2.332775 \\
\hline $\mathrm{C}$ & -1.157734 & -4.079025 & 2.937802 \\
\hline $\mathrm{C}$ & 0.165560 & -4.461559 & 2.829141 \\
\hline $\mathrm{H}$ & 1.323399 & -1.853652 & 0.959020 \\
\hline $\mathrm{C}$ & -1.186773 & -0.871827 & 0.856818 \\
\hline $\mathrm{C}$ & -3.002081 & -2.439471 & 2.499450 \\
\hline $\mathrm{H}$ & -1.830699 & -4.716115 & 3.498834 \\
\hline $\mathrm{H}$ & 0.522973 & -5.364572 & 3.307044 \\
\hline $\mathrm{C}$ & -3.438111 & -1.233302 & 1.939341 \\
\hline $\mathrm{C}$ & -2.525436 & -0.417710 & 1.275715 \\
\hline O & 2.383785 & -3.994514 & 1.947449 \\
\hline
\end{tabular}




\begin{tabular}{|c|c|c|c|}
\hline C & 4.523216 & -4.747553 & 2.636643 \\
\hline $\mathrm{H}$ & 4.846401 & -5.588181 & 3.254858 \\
\hline $\mathrm{H}$ & 4.574439 & -5.005981 & 1.577693 \\
\hline $\mathrm{H}$ & 5.198325 & -3.910596 & 2.828002 \\
\hline $\mathrm{C}$ & 3.134886 & -4.345985 & 3.048469 \\
\hline O & 2.722693 & -4.307347 & 4.181434 \\
\hline $\mathrm{C}$ & -4.872120 & -0.815513 & 2.129519 \\
\hline $\mathrm{C}$ & -5.378459 & -1.194935 & 3.532934 \\
\hline C & -5.297319 & 0.651024 & 1.937587 \\
\hline $\mathrm{H}$ & -5.321333 & 0.934428 & 0.881350 \\
\hline C & -6.700119 & 0.685350 & 2.587917 \\
\hline $\mathrm{H}$ & -7.501770 & 0.558661 & 1.849931 \\
\hline $\mathrm{C}$ & -6.754670 & -0.523916 & 3.543085 \\
\hline $\mathrm{H}$ & -6.913413 & 1.615937 & 3.123311 \\
\hline $\mathrm{H}$ & -4.604283 & 1.329029 & 2.440792 \\
\hline $\mathrm{C}$ & -5.359174 & -2.717009 & 3.629733 \\
\hline $\mathrm{C}$ & -3.927930 & -3.260867 & 3.383064 \\
\hline $\mathrm{H}$ & -3.986377 & -4.280732 & 2.981298 \\
\hline $\mathrm{H}$ & -3.412224 & -3.360381 & 4.347276 \\
\hline $\mathrm{H}$ & -2.852600 & 0.517102 & 0.839919 \\
\hline $\mathrm{H}$ & -1.406059 & -1.249234 & -0.160667 \\
\hline $\mathrm{C}$ & -3.765148 & 3.125836 & -1.175996 \\
\hline $\mathrm{C}$ & -4.142884 & 3.306567 & 0.157969 \\
\hline $\mathrm{C}$ & -4.658059 & 3.556412 & -2.159303 \\
\hline $\mathrm{C}$ & -5.375331 & 3.845638 & 0.511414 \\
\hline C & -5.890147 & 4.121551 & -1.830866 \\
\hline $\mathrm{C}$ & -6.250760 & 4.260884 & -0.490927 \\
\hline $\mathrm{C}$ & 2.954374 & 4.781773 & -0.408911 \\
\hline $\mathrm{C}$ & 3.082464 & 5.805887 & -1.354014 \\
\hline $\mathrm{C}$ & 3.544272 & 4.988502 & 0.842528 \\
\hline C & 3.767034 & 6.985900 & -1.071215 \\
\hline $\mathrm{C}$ & 4.241002 & 6.155716 & 1.144718 \\
\hline $\mathrm{C}$ & 4.349801 & 7.158653 & 0.182977 \\
\hline C & 4.564084 & -1.986519 & -0.944657 \\
\hline C & 5.153933 & -2.188179 & 0.305147 \\
\hline C & 5.241617 & -2.495412 & -2.058255 \\
\hline C & 6.386021 & -2.818236 & 0.447295 \\
\hline $\mathrm{C}$ & 6.459862 & -3.162984 & -1.936929 \\
\hline C & 7.037448 & -3.319413 & -0.677689 \\
\hline C & -2.040784 & -3.411798 & -2.725507 \\
\hline $\mathrm{C}$ & -1.942181 & -4.135371 & -3.916032 \\
\hline $\mathrm{C}$ & -2.887351 & -3.918685 & -1.734876 \\
\hline C & -2.657749 & -5.316050 & -4.116174 \\
\hline $\mathrm{C}$ & -3.615190 & -5.090474 & -1.911140 \\
\hline
\end{tabular}




$\begin{array}{rrr}-3.497479 & -5.792175 & -3.110510 \\ 4.534740 & -1.757247 & 1.415098 \\ 6.940135 & -2.960917 & 1.662653 \\ 4.726374 & -2.338904 & -3.280152 \\ 7.083941 & -3.639569 & -3.017618 \\ 8.209287 & -3.944231 & -0.549279 \\ -3.015614 & -3.265604 & -0.563078 \\ -1.138567 & -3.705111 & -4.892235 \\ -2.546479 & -5.991295 & -5.263258 \\ -4.416451 & -5.547914 & -0.940843 \\ -4.187246 & -6.919775 & -3.293061 \\ 2.531323 & 5.668505 & -2.564429 \\ 3.457146 & 4.046267 & 1.792761 \\ 4.798208 & 6.322388 & 2.348967 \\ 3.872844 & 7.948144 & -1.993038 \\ 5.012084 & 8.283219 & 0.463031 \\ -4.352152 & 3.402756 & -3.452396 \\ -6.731341 & 4.520820 & -2.789571 \\ -7.437557 & 4.779437 & -0.169260 \\ -3.321395 & 2.901397 & 1.138374 \\ -5.734671 & 3.931645 & 1.798728 \\ -6.048466 & -3.128203 & 2.882785 \\ -5.716747 & -3.059801 & 4.606040 \\ -4.561905 & -0.531168 & 4.670063 \\ -4.615561 & 0.561304 & 4.630874 \\ -4.960390 & -0.852555 & 5.637794 \\ -3.500866 & -0.794072 & 4.616909 \\ -7.718445 & -0.885588 & 4.180288 \\ -5.470770 & -1.407930 & 1.416113\end{array}$

\section{Structure: ${ }^{3}$ TS11}

$\begin{array}{llll}\mathrm{C} & 0.313838 & -2.249981 & -2.149364 \\ \mathrm{C} & 1.403116 & -2.914712 & -2.791920 \\ \mathrm{C} & 2.455407 & -2.038749 & -2.836439 \\ \mathrm{C} & 2.037688 & -0.824194 & -2.206739 \\ \mathrm{~N} & 0.732009 & -0.981682 & -1.736469 \\ \mathrm{H} & 1.361740 & -3.908537 & -3.215009 \\ \mathrm{H} & 3.416447 & -2.200865 & -3.303472 \\ \mathrm{C} & 0.953173 & 3.277620 & -1.199405 \\ \mathrm{C} & 2.301307 & 3.805457 & -1.170818 \\ \mathrm{C} & 3.136426 & 2.770520 & -1.448096 \\ \mathrm{C} & 2.306303 & 1.603428 & -1.673845 \\ \mathrm{~N} & 0.992522 & 1.941637 & -1.495587\end{array}$




\begin{tabular}{|c|c|c|c|}
\hline $\mathrm{H}$ & 2.570012 & 4.832300 & -0.971302 \\
\hline $\mathrm{H}$ & 4.215104 & 2.794305 & -1.501548 \\
\hline $\mathrm{C}$ & -3.447979 & -0.372200 & -1.021063 \\
\hline C & -4.247267 & -1.577517 & -1.049986 \\
\hline C & -3.421449 & -2.591724 & -1.414697 \\
\hline C & -2.110274 & -2.017273 & -1.620066 \\
\hline $\mathrm{N}$ & -2.157959 & -0.667652 & -1.376290 \\
\hline $\mathrm{H}$ & -5.301725 & -1.637825 & -0.825243 \\
\hline $\mathrm{H}$ & -3.671538 & -3.637121 & -1.513558 \\
\hline $\mathrm{C}$ & -3.188297 & 2.086807 & -0.716241 \\
\hline C & -3.698313 & 3.393935 & -0.370313 \\
\hline C & -2.670091 & 4.270522 & -0.500684 \\
\hline $\mathrm{C}$ & -1.509684 & 3.508119 & -0.900291 \\
\hline $\mathrm{N}$ & -1.860896 & 2.191649 & -1.053674 \\
\hline $\mathrm{H}$ & -4.706615 & 3.610476 & -0.052020 \\
\hline $\mathrm{H}$ & -2.684241 & 5.330946 & -0.298569 \\
\hline $\mathrm{C}$ & -0.975059 & -2.761915 & -1.986411 \\
\hline $\mathrm{C}$ & -0.205435 & 4.023864 & -0.950228 \\
\hline C & 2.800027 & 0.340535 & -2.066552 \\
\hline $\mathrm{C}$ & -3.928417 & 0.895662 & -0.661562 \\
\hline Mn & -0.624656 & 0.690775 & -1.491069 \\
\hline $\mathrm{Cl}$ & -0.873827 & 0.867522 & -3.723439 \\
\hline $\mathrm{C}$ & -5.315309 & 0.946839 & -0.120104 \\
\hline $\mathrm{C}$ & -5.641668 & 0.259005 & 1.056294 \\
\hline $\mathrm{C}$ & -6.348476 & 1.642319 & -0.755837 \\
\hline $\mathrm{C}$ & -6.934037 & 0.256457 & 1.571436 \\
\hline $\mathrm{C}$ & -7.645379 & 1.668599 & -0.246523 \\
\hline $\mathrm{C}$ & -7.940475 & 0.967341 & 0.921162 \\
\hline $\mathrm{C}$ & -1.138097 & -4.225254 & -2.205642 \\
\hline $\mathrm{C}$ & -0.483318 & -5.139171 & -1.371670 \\
\hline $\mathrm{C}$ & -1.934332 & -4.751770 & -3.228283 \\
\hline $\mathrm{C}$ & -0.618997 & -6.514835 & -1.531831 \\
\hline $\mathrm{C}$ & -2.087864 & -6.125358 & -3.406578 \\
\hline C & -1.426418 & -7.008886 & -2.554947 \\
\hline $\mathrm{C}$ & 4.249034 & 0.227656 & -2.385071 \\
\hline $\mathrm{C}$ & 4.875733 & 1.001319 & -3.370696 \\
\hline $\mathrm{C}$ & 5.057800 & -0.683478 & -1.697929 \\
\hline C & 6.243076 & 0.902418 & -3.625744 \\
\hline $\mathrm{C}$ & 6.423751 & -0.796416 & -1.926212 \\
\hline $\mathrm{C}$ & 7.021617 & 0.002333 & -2.899222 \\
\hline C & -0.027496 & 5.458972 & -0.588464 \\
\hline $\mathrm{C}$ & -0.497939 & 6.486699 & -1.410744 \\
\hline $\mathrm{C}$ & 0.597703 & 5.829569 & 0.607787 \\
\hline $\mathrm{C}$ & -0.363028 & 7.829028 & -1.062972 \\
\hline
\end{tabular}




\begin{tabular}{|c|c|c|c|}
\hline$y$ & 0.750572 & 7.165206 & 0.971849 \\
\hline C & 0.265308 & 8.167547 & 0.133935 \\
\hline $\mathrm{F}$ & -4.714246 & -0.457566 & 1.70418 \\
\hline F & -7.214929 & -0.441771 & 2.68161 \\
\hline $\mathrm{F}$ & -9.182131 & 0.971580 & 1.41066 \\
\hline $\mathrm{F}$ & -8.609117 & 2.346846 & -0.8781 \\
\hline $\mathrm{F}$ & -6.104314 & 2.311356 & -1.8900 \\
\hline $\mathrm{F}$ & -1.110765 & 6.188972 & -2.56341 \\
\hline $\mathrm{F}$ & -0.826079 & 8.790712 & -1.8684 \\
\hline $\mathrm{F}$ & 0.405352 & 9.450475 & 0.4765 \\
\hline $\mathrm{F}$ & 1.357408 & 7.491143 & 2.11892 \\
\hline$F$ & 1.088495 & 4.895971 & 1.43349 \\
\hline $\mathrm{F}$ & 4.165968 & 1.871403 & -4.09486 \\
\hline $\mathrm{F}$ & 6.811796 & 1.659969 & -4.56745 \\
\hline $\mathrm{F}$ & 8.331038 & -0.093498 & -3.13328 \\
\hline $\mathrm{F}$ & 7.164087 & -1.653912 & -1.2088 \\
\hline $\mathrm{F}$ & 4.512694 & -1.473851 & $-0.7545 \varepsilon$ \\
\hline $\mathrm{F}$ & 0.303922 & -4.695743 & -0.3792 \\
\hline $\mathrm{F}$ & 0.015500 & -7.361313 & -0.7120 \\
\hline $\mathrm{F}$ & -1.565179 & -8.326126 & -2.71919 \\
\hline $\mathrm{F}$ & -2.856564 & -6.601004 & -4.39118 \\
\hline $\mathrm{F}$ & -2.580011 & -3.930259 & -4.0632 \\
\hline $\mathrm{C}$ & -1.911235 & -3.322967 & 1.9149 \\
\hline $\mathrm{C}$ & -1.346138 & -2.167722 & 1.4228 \\
\hline C & 0.037260 & -1.962954 & 1.5321 \\
\hline $\mathrm{C}$ & 0.853085 & -2.933738 & 2.1972 \\
\hline C & 0.229533 & -4.138937 & 2.6339 \\
\hline $\mathrm{C}$ & -1.125398 & -4.338771 & 2.4974 \\
\hline $\mathrm{H}$ & -1.975145 & -1.394049 & 1.0123 \\
\hline C & 0.655768 & -0.747421 & 0.9823 \\
\hline $\mathrm{C}$ & 2.214482 & -2.621578 & 2.51820 \\
\hline $\mathrm{H}$ & 0.823546 & -4.921898 & 3.0891 \\
\hline $\mathrm{H}$ & -1.595636 & -5.247803 & 2.8509 \\
\hline C & 2.784199 & -1.403288 & 2.0958 \\
\hline $\mathrm{C}$ & 2.019625 & -0.507356 & 1.3590 \\
\hline O & -3.281896 & -3.456190 & 1.75745 \\
\hline $\mathrm{C}$ & -5.521870 & -3.576574 & $2.4910^{\circ}$ \\
\hline $\mathrm{H}$ & -6.129629 & -4.113536 & 3.2207 \\
\hline $\mathrm{H}$ & -5.798319 & -2.517203 & $2.5082 \varepsilon$ \\
\hline $\mathrm{H}$ & -5.698447 & -3.956640 & 1.481582 \\
\hline $\mathrm{C}$ & -4.068739 & -3.692722 & 2.86609 \\
\hline O & -3.624270 & -3.921568 & 3.9620 \\
\hline 0 & 4.123994 & -0.996389 & 2.6601 \\
\hline & 5.060088 & -2.187677 & 2.92592 \\
\hline
\end{tabular}




\begin{tabular}{|c|c|c|c|}
\hline $\mathrm{C}$ & 5.007596 & 0.078714 & 2.002652 \\
\hline $\mathrm{H}$ & 4.566817 & 1.077399 & 2.078162 \\
\hline C & 6.325049 & -0.049359 & 2.806276 \\
\hline $\mathrm{H}$ & 6.381561 & 0.678598 & 3.624582 \\
\hline C & 6.304492 & -1.457765 & 3.436714 \\
\hline 0 & 7.123204 & -1.897445 & 4.213070 \\
\hline $\mathrm{C}$ & 5.490565 & -2.954849 & 1.653463 \\
\hline $\mathrm{H}$ & 6.106036 & -3.812925 & 1.943484 \\
\hline $\mathrm{H}$ & 4.623524 & -3.316819 & 1.092451 \\
\hline $\mathrm{H}$ & 6.078117 & -2.339922 & 0.968339 \\
\hline $\mathrm{H}$ & 7.231036 & 0.082427 & 2.205518 \\
\hline $\mathrm{H}$ & 5.165395 & -0.136493 & 0.946020 \\
\hline $\mathrm{C}$ & 4.364320 & -3.111059 & 3.920916 \\
\hline $\mathrm{C}$ & 3.021859 & -3.613888 & 3.344614 \\
\hline $\mathrm{H}$ & 4.178877 & -2.558955 & 4.850873 \\
\hline $\mathrm{H}$ & 4.997828 & -3.964346 & 4.184209 \\
\hline $\mathrm{H}$ & 2.401829 & -3.976655 & 4.170902 \\
\hline $\mathrm{H}$ & 3.199513 & -4.492059 & 2.706519 \\
\hline $\mathrm{H}$ & 2.442109 & 0.437726 & 1.036440 \\
\hline $\mathrm{H}$ & 0.861434 & -1.073243 & -0.238407 \\
\hline $\mathrm{H}$ & 3.879607 & -0.588507 & 3.653528 \\
\hline S & -0.833268 & 1.209857 & 1.952973 \\
\hline $\mathrm{C}$ & 0.346153 & 0.990333 & 3.303761 \\
\hline $\mathrm{C}$ & 1.428977 & 1.870196 & 3.396898 \\
\hline $\mathrm{C}$ & 0.185438 & -0.049059 & 4.218100 \\
\hline $\mathrm{C}$ & 2.367567 & 1.685016 & 4.410047 \\
\hline $\mathrm{H}$ & 1.511281 & 2.692607 & 2.692762 \\
\hline C & 1.140557 & -0.226978 & 5.220168 \\
\hline $\mathrm{H}$ & -0.678239 & -0.701053 & 4.141143 \\
\hline C & 2.245399 & 0.629280 & 5.328962 \\
\hline $\mathrm{H}$ & 3.205833 & 2.372919 & 4.495823 \\
\hline $\mathrm{H}$ & 1.018906 & -1.037148 & 5.935259 \\
\hline $\mathrm{C}$ & 3.297328 & 0.408912 & 6.391158 \\
\hline $\mathrm{H}$ & 4.139705 & -0.179505 & 6.000053 \\
\hline $\mathrm{H}$ & 3.708964 & 1.357695 & 6.751429 \\
\hline $\mathrm{H}$ & 2.893968 & -0.135505 & 7.250857 \\
\hline O & -0.865061 & 2.651461 & 1.658986 \\
\hline O & -2.097144 & 0.576020 & 2.385685 \\
\hline & -0.13586 & 0.419348 & 0.676748 \\
\hline
\end{tabular}

Structure: ${ }^{5}$ TS11

\begin{tabular}{cccc} 
C & 0.363708 & -2.262450 & -2.179223 \\
C & 1.440755 & -2.927571 & -2.855902 \\
\multicolumn{2}{c}{$\mathrm{s} 181$} & &
\end{tabular}




\begin{tabular}{|c|c|c|c|}
\hline C & 2.499570 & -2.060158 & -2.881490 \\
\hline $\mathrm{C}$ & 2.085277 & -0.859554 & -2.208644 \\
\hline $\mathrm{N}$ & 0.796698 & -1.025295 & -1.740230 \\
\hline $\mathrm{H}$ & 1.380158 & -3.903944 & -3.316209 \\
\hline $\mathrm{H}$ & 3.453652 & -2.208055 & -3.367778 \\
\hline C & 0.946397 & 3.275242 & -1.223131 \\
\hline C & 2.295150 & 3.794264 & -1.220373 \\
\hline $\mathrm{C}$ & 3.131297 & 2.756084 & -1.484284 \\
\hline $\mathrm{C}$ & 2.312652 & 1.581926 & -1.685619 \\
\hline $\mathrm{N}$ & 0.994558 & 1.929925 & -1.503576 \\
\hline $\mathrm{H}$ & 2.566892 & 4.824335 & -1.044981 \\
\hline $\mathrm{H}$ & 4.209026 & 2.785163 & -1.545442 \\
\hline C & -3.394809 & -0.286505 & -1.056692 \\
\hline C & -4.199215 & -1.483233 & -1.119393 \\
\hline $\mathrm{C}$ & -3.383765 & -2.503894 & -1.491561 \\
\hline $\mathrm{C}$ & -2.063001 & -1.955120 & -1.673754 \\
\hline $\mathrm{N}$ & -2.101062 & -0.601845 & -1.409264 \\
\hline $\mathrm{H}$ & -5.257294 & -1.535078 & -0.911758 \\
\hline $\mathrm{H}$ & -3.651000 & -3.542666 & -1.610422 \\
\hline $\mathrm{C}$ & -3.189437 & 2.179087 & -0.690977 \\
\hline C & -3.687547 & 3.480075 & -0.282502 \\
\hline $\mathrm{C}$ & -2.654964 & 4.352378 & -0.411554 \\
\hline $\mathrm{C}$ & -1.512841 & 3.585268 & -0.875069 \\
\hline $\mathrm{N}$ & -1.884529 & 2.291035 & -1.063829 \\
\hline $\mathrm{H}$ & -4.684058 & 3.691719 & 0.075450 \\
\hline $\mathrm{H}$ & -2.651770 & 5.405220 & -0.170656 \\
\hline $\mathrm{C}$ & -0.944970 & -2.731870 & -2.031085 \\
\hline $\mathrm{C}$ & -0.192159 & 4.054512 & -0.953938 \\
\hline $\mathrm{C}$ & 2.826408 & 0.321121 & -2.062086 \\
\hline $\mathrm{C}$ & -3.899296 & 0.968840 & -0.667062 \\
\hline $\mathrm{Mn}$ & -0.575633 & 0.700028 & -1.482945 \\
\hline $\mathrm{Cl}$ & -0.730876 & 0.805070 & -3.827240 \\
\hline $\mathrm{C}$ & -5.286384 & 0.987874 & -0.124778 \\
\hline C & -5.617945 & 0.265617 & 1.029646 \\
\hline $\mathrm{C}$ & -6.314722 & 1.714934 & -0.734062 \\
\hline $\mathrm{C}$ & -6.908945 & 0.262422 & 1.548257 \\
\hline C & -7.610278 & 1.738291 & -0.221234 \\
\hline $\mathrm{C}$ & -7.909888 & 1.003809 & 0.924375 \\
\hline $\mathrm{C}$ & -1.145230 & -4.189434 & -2.256509 \\
\hline $\mathrm{C}$ & -0.519033 & -5.117271 & -1.415668 \\
\hline $\mathrm{C}$ & -1.935472 & -4.698353 & -3.292607 \\
\hline $\mathrm{C}$ & -0.675965 & -6.489787 & -1.582343 \\
\hline C & -2.109787 & -6.068725 & -3.477505 \\
\hline $\mathrm{C}$ & -1.475937 & -6.966303 & -2.619505 \\
\hline
\end{tabular}




\begin{tabular}{|c|c|c|c|}
\hline C & 4.280729 & 0.218310 & -2.358042 \\
\hline $\mathrm{C}$ & 4.924002 & 0.978384 & -3.343393 \\
\hline $\mathrm{C}$ & 5.076501 & -0.687242 & -1.648432 \\
\hline C & 6.295318 & 0.873526 & -3.574617 \\
\hline C & 6.445712 & -0.804952 & -1.851829 \\
\hline C & 7.060451 & -0.019295 & -2.825018 \\
\hline $\mathrm{C}$ & 0.031309 & 5.480771 & -0.582545 \\
\hline $\mathrm{C}$ & -0.455748 & 6.525072 & -1.374352 \\
\hline $\mathrm{C}$ & 0.697420 & 5.829084 & 0.598909 \\
\hline $\mathrm{C}$ & -0.294705 & 7.861139 & -1.013603 \\
\hline $\mathrm{C}$ & 0.876398 & 7.158066 & 0.974898 \\
\hline C & 0.375537 & 8.176864 & 0.166514 \\
\hline $\mathrm{F}$ & -4.698795 & -0.482911 & 1.653375 \\
\hline $\mathrm{F}$ & -7.195423 & -0.465724 & 2.638512 \\
\hline $\mathrm{F}$ & -9.149797 & 1.005492 & 1.418227 \\
\hline $\mathrm{F}$ & -8.568229 & 2.446058 & -0.828588 \\
\hline $\mathrm{F}$ & -6.067498 & 2.417937 & -1.846324 \\
\hline $\mathrm{F}$ & -1.109223 & 6.250567 & -2.509991 \\
\hline $\mathrm{F}$ & -0.773290 & 8.838385 & -1.790828 \\
\hline $\mathrm{F}$ & 0.540720 & 9.453490 & 0.521464 \\
\hline $\mathrm{F}$ & 1.522471 & 7.462240 & 2.106726 \\
\hline $\mathrm{F}$ & 1.203367 & 4.880237 & 1.397357 \\
\hline $\mathrm{F}$ & 4.227958 & 1.841017 & -4.088772 \\
\hline $\mathrm{F}$ & 6.880995 & 1.618463 & -4.515963 \\
\hline $\mathrm{F}$ & 8.373340 & -0.119798 & -3.036755 \\
\hline $\mathrm{F}$ & 7.172068 & -1.654987 & -1.111266 \\
\hline $\mathrm{F}$ & 4.514465 & -1.465441 & -0.704181 \\
\hline $\mathrm{F}$ & 0.257502 & -4.690582 & -0.406738 \\
\hline $\mathrm{F}$ & -0.069486 & -7.349890 & -0.755320 \\
\hline $\mathrm{F}$ & -1.634992 & -8.280443 & -2.790361 \\
\hline $\mathrm{F}$ & -2.872260 & -6.528266 & -4.474446 \\
\hline $\mathrm{F}$ & -2.555217 & -3.863278 & -4.132887 \\
\hline $\mathrm{C}$ & -1.976839 & -3.293551 & 1.872196 \\
\hline C & -1.387718 & -2.142512 & 1.394322 \\
\hline $\mathrm{C}$ & -0.005421 & -1.957614 & 1.529666 \\
\hline $\mathrm{C}$ & 0.785033 & -2.938208 & 2.209705 \\
\hline $\mathrm{C}$ & 0.139426 & -4.136929 & 2.631111 \\
\hline $\mathrm{C}$ & -1.214491 & -4.320910 & 2.466016 \\
\hline $\mathrm{H}$ & -1.998842 & -1.358647 & 0.975546 \\
\hline $\mathrm{C}$ & 0.644048 & -0.755033 & 0.976248 \\
\hline $\mathrm{C}$ & 2.143071 & -2.642942 & 2.557539 \\
\hline $\mathrm{H}$ & 0.714499 & -4.927536 & 3.097176 \\
\hline $\mathrm{H}$ & -1.701517 & -5.225823 & 2.806421 \\
\hline $\mathrm{C}$ & 2.735829 & -1.425328 & 2.157233 \\
\hline
\end{tabular}




\begin{tabular}{|c|c|c|c|}
\hline C & 2.001295 & -0.523065 & 1.402690 \\
\hline O & -3.343238 & -3.410488 & 1.684404 \\
\hline $\mathrm{C}$ & -5.600792 & -3.581008 & 2.352126 \\
\hline $\mathrm{H}$ & -6.218062 & -4.161850 & 3.039056 \\
\hline $\mathrm{H}$ & -5.898817 & -2.529267 & 2.410762 \\
\hline $\mathrm{H}$ & -5.741070 & -3.917355 & 1.321819 \\
\hline $\mathrm{C}$ & -4.157652 & -3.686483 & 2.764666 \\
\hline O & -3.739937 & -3.936138 & 3.866503 \\
\hline C & 4.075086 & -1.046934 & 2.740582 \\
\hline $\mathrm{C}$ & 4.990875 & -2.258348 & 2.986815 \\
\hline $\mathrm{C}$ & 4.977362 & 0.023894 & 2.101619 \\
\hline $\mathrm{H}$ & 4.555508 & 1.029001 & 2.197583 \\
\hline $\mathrm{C}$ & 6.295628 & -0.143296 & 2.896906 \\
\hline $\mathrm{H}$ & 6.373322 & 0.572480 & 3.724043 \\
\hline C & 6.248445 & -1.559250 & 3.508226 \\
\hline O & 7.058253 & -2.025663 & 4.278069 \\
\hline $\mathrm{C}$ & 5.407646 & -3.010362 & 1.700518 \\
\hline $\mathrm{H}$ & 6.003986 & -3.886771 & 1.975176 \\
\hline $\mathrm{H}$ & 4.534830 & -3.343020 & 1.130169 \\
\hline $\mathrm{H}$ & 6.009815 & -2.394945 & 1.028595 \\
\hline $\mathrm{H}$ & 7.200553 & -0.023558 & 2.292026 \\
\hline $\mathrm{H}$ & 5.125890 & -0.174300 & 1.040211 \\
\hline $\mathrm{C}$ & 4.278606 & -3.185265 & 3.966388 \\
\hline $\mathrm{C}$ & 2.927330 & -3.654674 & 3.381893 \\
\hline $\mathrm{H}$ & 4.103070 & -2.645763 & 4.905437 \\
\hline $\mathrm{H}$ & 4.896057 & -4.054461 & 4.215115 \\
\hline $\mathrm{H}$ & 2.295679 & -4.011007 & 4.202244 \\
\hline $\mathrm{H}$ & 3.088492 & -4.531090 & 2.737059 \\
\hline $\mathrm{H}$ & 2.445089 & 0.412359 & 1.079927 \\
\hline $\mathrm{H}$ & 0.884097 & -1.102689 & -0.190791 \\
\hline $\mathrm{H}$ & 3.831381 & -0.652961 & 3.740083 \\
\hline S & -0.816823 & 1.237032 & 1.900781 \\
\hline C & 0.277316 & 0.934457 & 3.306961 \\
\hline C & 1.393267 & 1.761573 & 3.476678 \\
\hline $\mathrm{C}$ & 0.019136 & -0.110171 & 4.192404 \\
\hline $\mathrm{C}$ & 2.268247 & 1.512895 & 4.531650 \\
\hline $\mathrm{H}$ & 1.546760 & 2.595262 & 2.797986 \\
\hline $\mathrm{C}$ & 0.913670 & -0.354109 & 5.236161 \\
\hline $\mathrm{H}$ & -0.870649 & -0.716919 & 4.062277 \\
\hline $\mathrm{C}$ & 2.051009 & 0.444392 & 5.418037 \\
\hline $\mathrm{H}$ & 3.131403 & 2.158962 & 4.676225 \\
\hline $\mathrm{H}$ & 0.717746 & -1.170942 & 5.926660 \\
\hline $\mathrm{C}$ & 3.036099 & 0.157601 & 6.527079 \\
\hline $\mathrm{H}$ & 3.930742 & -0.351863 & 6.141792 \\
\hline
\end{tabular}




$\begin{array}{rrrr}\mathrm{H} & 3.377183 & 1.080794 & 7.008328 \\ \mathrm{H} & 2.600277 & -0.486120 & 7.297505 \\ \mathrm{O} & -0.737665 & 2.681597 & 1.628256 \\ \mathrm{O} & -2.139643 & 0.682789 & 2.263044 \\ \mathrm{~N} & -0.130913 & 0.413559 & 0.639795\end{array}$

\section{Structure: ${ }^{3}$ INT10}

\begin{tabular}{|c|c|c|c|}
\hline $\mathrm{C}$ & -1.299142 & 3.289776 & 0.690606 \\
\hline C & -1.133934 & 4.649916 & 1.035632 \\
\hline C & 0.217067 & 4.946448 & $1.0180 \varepsilon$ \\
\hline C & 0.924954 & 3.790925 & 0.62526 \\
\hline $\mathrm{N}$ & -0.022822 & 2.760361 & 0.35990 \\
\hline $\mathrm{H}$ & -1.933116 & 5.301821 & 1.3600 \\
\hline $\mathrm{H}$ & 0.676428 & 5.879588 & 1.3124 \\
\hline C & 3.434377 & 0.236184 & $0.4087^{\circ}$ \\
\hline C & 4.680663 & 0.935266 & 0.1802 \\
\hline C & 4.384655 & 2.260069 & 0.1457 \\
\hline C & 2.954171 & 2.383210 & 0.3402 \\
\hline $\mathrm{N}$ & 2.399601 & 1.131355 & $0.4557^{\circ}$ \\
\hline $\mathrm{H}$ & 5.648457 & 0.470741 & 0.0638 \\
\hline $\mathrm{H}$ & 5.066751 & 3.083814 & -0.0052 \\
\hline C & -2.036555 & -0.928987 & 1.5994 \\
\hline C & -3.462454 & -0.785951 & 1.7950 \\
\hline $\mathrm{C}$ & -3.784183 & 0.485909 & 1.4490 \\
\hline C & -2.550138 & 1.156258 & 1.0870 \\
\hline $\mathrm{N}$ & -1.512231 & 0.264362 & 1.1735 \\
\hline $\mathrm{H}$ & -4.131396 & -1.557775 & $2.1433^{\circ}$ \\
\hline $\mathrm{H}$ & -4.764490 & 0.936046 & 1.4641 \\
\hline C & 0.062049 & -2.258396 & 1.5440 \\
\hline C & 0.802045 & -3.495476 & 1.6656 \\
\hline $\mathrm{C}$ & 2.092646 & -3.219689 & 1.3433 \\
\hline C & 2.159143 & -1.816146 & 1.0099 \\
\hline $\mathrm{N}$ & 0.907505 & -1.263553 & 1.12489 \\
\hline $\mathrm{H}$ & 0.385712 & -4.460606 & 1.9075 \\
\hline $\mathrm{H}$ & 2.925281 & -3.906984 & 1.3235 \\
\hline C & -2.469690 & 2.539325 & 0.8306 \\
\hline $\mathrm{C}$ & 3.329087 & -1.141908 & 0.6369 \\
\hline C & 2.298092 & 3.623444 & 0.4757 \\
\hline $\mathrm{C}$ & -1.312968 & -2.115303 & 1.7930 \\
\hline Mn & 0.439758 & 0.623553 & 0.8045 \\
\hline $\mathrm{Cl}$ & 0.722599 & 1.184648 & 2.98928 \\
\hline $\mathrm{C}$ & -2.038159 & -3.312202 & 2.3097 \\
\hline$C$ & -3.062748 & -3.937372 & 1.5927 \\
\hline
\end{tabular}




\begin{tabular}{|c|c|c|c|}
\hline C & -1.702530 & -3.874865 & 3.548029 \\
\hline $\mathrm{C}$ & -3.720307 & -5.068837 & 2.065033 \\
\hline $\mathrm{C}$ & -2.341188 & -5.010393 & 4.040948 \\
\hline C & -3.354540 & -5.610601 & 3.295202 \\
\hline C & -3.736410 & 3.323349 & 0.782903 \\
\hline C & -4.141745 & 3.882957 & -0.433557 \\
\hline $\mathrm{C}$ & -4.564667 & 3.524002 & 1.890579 \\
\hline $\mathrm{C}$ & -5.346092 & 4.566495 & -0.566519 \\
\hline $\mathrm{C}$ & -5.768279 & 4.218931 & 1.787005 \\
\hline $\mathrm{C}$ & -6.159529 & 4.738395 & 0.553116 \\
\hline $\mathrm{C}$ & 3.123503 & 4.861082 & 0.524555 \\
\hline $\mathrm{C}$ & 4.055766 & 5.087566 & 1.544348 \\
\hline $\mathrm{C}$ & 2.976429 & 5.859995 & -0.446165 \\
\hline C & 4.822121 & 6.249511 & 1.593229 \\
\hline $\mathrm{C}$ & 3.728907 & 7.031736 & -0.413000 \\
\hline $\mathrm{C}$ & 4.655774 & 7.224251 & 0.610293 \\
\hline $\mathrm{C}$ & 4.581462 & -1.946402 & 0.532877 \\
\hline $\mathrm{C}$ & 5.583911 & -1.873928 & 1.504899 \\
\hline C & 4.801242 & -2.796867 & -0.552189 \\
\hline $\mathrm{C}$ & 6.762273 & -2.611704 & 1.402679 \\
\hline C & 5.968266 & -3.545008 & -0.672838 \\
\hline $\mathrm{C}$ & 6.953860 & -3.453015 & 0.307266 \\
\hline F & -3.456211 & -3.440457 & 0.410604 \\
\hline $\mathrm{F}$ & -4.694102 & -5.632949 & 1.342744 \\
\hline $\mathrm{F}$ & -3.974828 & -6.695366 & 3.760696 \\
\hline F & -1.993695 & -5.522926 & 5.224803 \\
\hline $\mathrm{F}$ & -0.735255 & -3.324990 & 4.291305 \\
\hline F & 5.428392 & -1.070948 & 2.562463 \\
\hline $\mathrm{F}$ & 7.704670 & -2.521753 & 2.345048 \\
\hline $\mathrm{F}$ & 8.075252 & -4.166451 & 0.198169 \\
\hline $\mathrm{F}$ & 6.144841 & -4.356183 & -1.728439 \\
\hline $\mathrm{F}$ & 3.872732 & -2.918334 & -1.508321 \\
\hline $\mathrm{F}$ & 4.229746 & 4.174395 & 2.506133 \\
\hline $\mathrm{F}$ & 5.705786 & 6.439695 & 2.577546 \\
\hline F & 5.382170 & 8.342089 & 0.650386 \\
\hline $\mathrm{F}$ & 3.571876 & 7.966980 & -1.354562 \\
\hline $\mathrm{F}$ & 2.095318 & 5.702136 & -1.439457 \\
\hline $\mathrm{F}$ & -3.377457 & 3.722574 & -1.523951 \\
\hline F & -5.727959 & 5.047107 & -1.752395 \\
\hline $\mathrm{F}$ & -7.313533 & 5.396693 & 0.443552 \\
\hline $\mathrm{F}$ & -6.545371 & 4.393998 & 2.859453 \\
\hline $\mathrm{F}$ & -4.216848 & 3.027957 & 3.084128 \\
\hline $\mathrm{C}$ & 0.395964 & -4.067516 & -1.760174 \\
\hline C & 0.284700 & -2.704787 & -1.646070 \\
\hline
\end{tabular}




\begin{tabular}{|c|c|c|c|}
\hline C & -0.982751 & -2.078683 & -1.781443 \\
\hline C & -2.133061 & -2.884223 & -2.090008 \\
\hline $\mathrm{C}$ & -1.957814 & -4.295825 & -2.163271 \\
\hline $\mathrm{C}$ & -0.728016 & -4.884936 & -1.999691 \\
\hline $\mathrm{H}$ & 1.163067 & -2.101541 & -1.463333 \\
\hline $\mathrm{C}$ & -1.147268 & -0.663719 & -1.641002 \\
\hline $\mathrm{C}$ & -3.409523 & -2.277849 & -2.313227 \\
\hline $\mathrm{H}$ & -2.813205 & -4.932378 & -2.355111 \\
\hline $\mathrm{H}$ & -0.609340 & -5.960373 & -2.057834 \\
\hline C & -3.519792 & -0.895322 & -2.209042 \\
\hline C & -2.396362 & -0.115096 & -1.862369 \\
\hline O & 1.676955 & -4.630691 & -1.730499 \\
\hline $\mathrm{C}$ & 3.325213 & -6.211325 & -1.146226 \\
\hline $\mathrm{H}$ & 3.247351 & -7.018663 & -1.884395 \\
\hline $\mathrm{H}$ & 3.992884 & -5.450543 & -1.549847 \\
\hline $\mathrm{H}$ & 3.724838 & -6.636868 & -0.223307 \\
\hline $\mathrm{C}$ & 1.942539 & -5.664766 & -0.884683 \\
\hline O & 1.167859 & -6.093069 & -0.059839 \\
\hline $\mathrm{C}$ & -4.843193 & -0.249393 & -2.541047 \\
\hline $\mathrm{C}$ & -6.039775 & -1.139160 & -2.147039 \\
\hline $\mathrm{C}$ & -5.214160 & 1.149936 & -2.018450 \\
\hline $\mathrm{H}$ & -4.637617 & 1.944691 & -2.495130 \\
\hline $\mathrm{C}$ & -6.725177 & 1.240183 & -2.337927 \\
\hline $\mathrm{H}$ & -6.909204 & 1.739774 & -3.296905 \\
\hline $\mathrm{C}$ & -7.216496 & -0.217553 & -2.467827 \\
\hline O & -8.339986 & -0.560414 & -2.764664 \\
\hline C & -6.131916 & -1.418472 & -0.625491 \\
\hline $\mathrm{H}$ & -6.999043 & -2.058330 & -0.429882 \\
\hline $\mathrm{H}$ & -5.232164 & -1.916844 & -0.258998 \\
\hline $\mathrm{H}$ & -6.259547 & -0.499947 & -0.043010 \\
\hline $\mathrm{H}$ & -7.317085 & 1.777248 & -1.588589 \\
\hline $\mathrm{H}$ & -5.032928 & 1.216096 & -0.941338 \\
\hline C & -5.939271 & -2.440761 & -2.936246 \\
\hline C & -4.615860 & -3.168804 & -2.600576 \\
\hline $\mathrm{H}$ & -5.976556 & -2.205909 & -4.007242 \\
\hline $\mathrm{H}$ & -6.791979 & -3.096712 & -2.729515 \\
\hline $\mathrm{H}$ & -4.381039 & -3.855263 & -3.423261 \\
\hline $\mathrm{H}$ & -4.768773 & -3.807996 & -1.719848 \\
\hline $\mathrm{H}$ & -2.500901 & 0.960932 & -1.783165 \\
\hline $\mathrm{H}$ & 0.084719 & 2.535370 & -0.664730 \\
\hline $\mathrm{H}$ & -4.880109 & -0.171272 & -3.641846 \\
\hline S & 0.556411 & 1.018840 & -2.606401 \\
\hline 0 & 2.022311 & 0.155524 & -3.169183 \\
\hline & 1.866295 & -1.008238 & -3.929292 \\
\hline
\end{tabular}




$\begin{array}{lrrr}\mathrm{C} & 3.288806 & 0.622304 & -2.832049 \\ \mathrm{C} & 2.995941 & -1.722260 & -4.314493 \\ \mathrm{H} & 0.874224 & -1.344838 & -4.209325 \\ \mathrm{C} & 4.411903 & -0.103439 & -3.231363 \\ \mathrm{H} & 3.388603 & 1.544602 & -2.275230 \\ \mathrm{C} & 4.284587 & -1.289975 & -3.962557 \\ \mathrm{H} & 2.877447 & -2.635412 & -4.892261 \\ \mathrm{H} & 5.401586 & 0.261760 & -2.966694 \\ \mathrm{C} & 5.494705 & -2.112476 & -4.333088 \\ \mathrm{H} & 5.399373 & -2.537462 & -5.338387 \\ \mathrm{H} & 6.413364 & -1.517238 & -4.302164 \\ \mathrm{H} & 5.616619 & -2.950718 & -3.636422 \\ \mathrm{O} & -0.383728 & 1.019428 & -3.735808 \\ \mathrm{O} & 1.022471 & 2.337292 & -2.081746 \\ \mathrm{~N} & -0.048571 & 0.193229 & -1.311257\end{array}$

\section{Structure: ${ }^{5}$ INT10}

C

C

C

C

$\mathrm{N}$

$\mathrm{H}$

$\mathrm{H}$

C

C

C

C

$\mathrm{N}$

$\mathrm{H}$

$\mathrm{H}$

C

C

C

C

$\mathrm{N}$

$\mathrm{H}$

$\mathrm{H}$

C

C

C

C

$\mathrm{N}$
1.374775

1.220481

$-0.123493$

$-0.827170$

0.113167

2. 011761

$-0.585973$

$-3.387015$

$-4.618462$

$-4.302025$

$-2.869766$

$-2.337637$

$-5.598948$

$-4.977213$

1.983039

3. 408285

3.762218

2.554434

1.500223

4.059973

4.753210

$-0.124004$

$-0.881909$

$-2.172430$

$-2.210537$

$-0.958945$
3.319546

4.655028

4.978688

3.862721

2. 860730

5.252660

5.882401

0.281577

1.006489

2. 325728

2. 439082

1.171486

0.561913

3.153608

$-0.962877$

$-0.836812$

0.427665

1.128972

0.247294

$-1.616311$

0.852934

$-2.321222$

$-3.557921$

$-3.258478$

$-1.843232$

$-1.324997$
$-0.633941$

$-1.085999$

$-1.032753$

$-0.519265$

$-0.225561$

$-1.516769$

$-1.403993$

$-0.418872$

$-0.199036$

$-0.120692$

$-0.277033$

$-0.406624$

$-0.119413$

0.037602

$-1.567399$

$-1.773479$

$-1.428033$

$-1.047376$

$-1.122464$

$-2.136603$

$-1.454496$

$-1.566768$

$-1.694253$

$-1.389662$

$-1.061221$

$-1.176168$ 


$$
\begin{array}{rrr}
-0.479050 & -4.531454 & -1.927452 \\
-3.016002 & -3.933286 & -1.376106 \\
2.516464 & 2.516070 & -0.794742 \\
-3.341165 & -1.102137 & -0.684408 \\
-2.205666 & 3.680475 & -0.378641 \\
1.257086 & -2.153976 & -1.782253 \\
-0.412321 & 0.641733 & -0.717160 \\
-0.634063 & 1.391961 & -2.919975 \\
1.995941 & -3.344982 & -2.295095 \\
3.011832 & -3.974671 & -1.569545 \\
1.668785 & -3.908659 & -3.535562 \\
3.670732 & -5.107707 & -2.035669 \\
2.308468 & -5.046274 & -4.022664 \\
3.313877 & -5.649222 & -3.268560 \\
3.805169 & 3.264985 & -0.795809 \\
4.251517 & 3.844252 & 0.397429 \\
4.607999 & 3.427961 & -1.928718 \\
5.468648 & 4.512409 & 0.484045 \\
5.824731 & 4.105144 & -1.870965 \\
6.255337 & 4.646097 & -0.659669 \\
-3.027633 & 4.919370 & -0.443811 \\
-3.941857 & 5.153353 & -1.478812 \\
-2.877963 & 5.923713 & 0.521381 \\
-4.690694 & 6.325838 & -1.544639 \\
-3.613993 & 7.105149 & 0.471677 \\
-4.523954 & 7.304255 & -0.565375 \\
-4.630677 & -1.850106 & -0.602542 \\
-5.624448 & -1.720478 & -1.577481 \\
-4.882666 & -2.725732 & 0.454997 \\
-6.825481 & -2.424197 & -1.501871 \\
-6.071018 & -3.443438 & 0.547593 \\
-7.048366 & -3.292192 & -0.433418 \\
3.395831 & -3.479947 & -0.383279 \\
4.636239 & -5.674333 & -1.303980 \\
3.934894 & -6.736049 & -3.728334 \\
1.969191 & -5.558330 & -5.209141 \\
0.709805 & -3.357084 & -4.288209 \\
-5.438363 & -0.893038 & -2.610964 \\
-7.759648 & -2.277641 & -2.445430 \\
-8.191352 & -3.974409 & -0.351146 \\
-6.275831 & -4.281629 & 1.576917 \\
-3.961605 & -2.905025 & 1.410319 \\
-4.113551 & 4.239921 & -2.440300 \\
-5.557022 & 6.523272 & -2.542886
\end{array}
$$




$$
\begin{aligned}
& -5.234114 \\
& -3.457015 \\
& -2.013677 \\
& \text { 3. } 513022 \\
& 5.886846 \\
& 7.421327 \\
& 6.576634 \\
& \text { 4. } 220898 \\
& -0.470184 \\
& -0.337895 \\
& 0.934976 \\
& \text { 2. } 071417 \\
& 1.875895 \\
& 0.640172 \\
& -1.206086 \\
& 1.119739 \\
& \text { 3. } 354597 \\
& \text { 2. } 720282 \\
& 0.507038 \\
& 3.484603 \\
& 2.373079 \\
& -1.759377 \\
& -3.427926 \\
& -3.386192 \\
& -4.099278 \\
& -3.800602 \\
& -2.031083 \\
& -1.250635 \\
& \text { 4. } 815926 \\
& 6.000279 \\
& 5.205617 \\
& 4.640765 \\
& 6.717922 \\
& 6.909578 \\
& 7.188908 \\
& 8.306677 \\
& 6.090331 \\
& 6.949327 \\
& 5.184978 \\
& 6.230673 \\
& 8.431733 \\
& -0.622071 \\
& 8.043785 \\
& 1.409889 \\
& 5.759365 \\
& \text { 1. } 528132 \\
& 3.721798 \\
& \text { 1. } 510551 \\
& \text { 5. } 017312 \\
& 1.647374 \\
& 5.288926 \\
& 4.244518 \\
& -0.594765 \\
& \text { 2. } 915243 \\
& -2.966369 \\
& -3.102648 \\
& -4.071134 \\
& \text { 1. } 745048 \\
& -2.710520 \\
& 1.626032 \\
& -2.100250 \\
& \text { 1. } 780159 \\
& -2.920253 \\
& 2.102090 \\
& -4.329072 \\
& \text { 2. } 177021 \\
& \text { 2. } 001892 \\
& \text { 1. } 428195 \\
& -2.097795 \\
& 1.648885 \\
& -0.687242 \\
& \text { 2. } 333511 \\
& -2.331197 \\
& 2.380806 \\
& \text { 2. } 063264 \\
& -5.975697 \\
& -0.949935 \\
& \text { 2. } 231360 \\
& 1.883876 \\
& 1.705634 \\
& -4.612275 \\
& 1.134524 \\
& -6.176077 \\
& 1.936085 \\
& -6.923486 \\
& 1.456208 \\
& -5.380008 \\
& 0.232710 \\
& -6.665784 \\
& 0.881293 \\
& -5.663250 \\
& 0.081639 \\
& -6.126505 \\
& 2.564561 \\
& -0.321534 \\
& 2.171816 \\
& -1.228030 \\
& 2.039394 \\
& 1.071969 \\
& \text { 2. } 514174 \\
& 2.358408 \\
& 1.141869 \\
& \text { 3. } 315389 \\
& 1.642348 \\
& 2.493334 \\
& -0.322155 \\
& 2.794094 \\
& 0.650221 \\
& 0.455494 \\
& -1.508355 \\
& -2.159346 \\
& 0.282263 \\
& 7.317099 \quad 1.667535 \\
& 0.068007 \\
& 5.024402 \\
& \text { 1. } 139288 \\
& 1.606776 \\
& 5.880920-2.527995 \\
& 0.962301 \\
& 4.547986-3.238111 \\
& 2.961189 \\
& 2.624237
\end{aligned}
$$




$\begin{array}{lrrr}\mathrm{H} & 5.920368 & -2.293461 & 4.032167 \\ \mathrm{H} & 6.724573 & -3.195782 & 2.755387 \\ \mathrm{H} & 4.302132 & -3.920337 & 3.447205 \\ \mathrm{H} & 4.693262 & -3.880066 & 1.744299 \\ \mathrm{H} & 2.491552 & 0.920638 & 1.811969 \\ \mathrm{H} & 0.004942 & 2.556514 & 0.765089 \\ \mathrm{H} & 4.853389 & -0.242624 & 3.665260 \\ \mathrm{~S} & -0.585521 & 0.977338 & 2.642039 \\ \mathrm{C} & -2.055165 & 0.098680 & 3.169111 \\ \mathrm{C} & -1.909650 & -1.079433 & 3.908414 \\ \mathrm{C} & -3.317285 & 0.580152 & 2.834801 \\ \mathrm{C} & -3.045632 & -1.795317 & 4.271621 \\ \mathrm{H} & -0.921516 & -1.425523 & 4.190154 \\ \mathrm{C} & -4.446267 & -0.146831 & 3.213149 \\ \mathrm{H} & -3.409271 & 1.515366 & 2.298694 \\ \mathrm{C} & -4.329543 & -1.349034 & 3.920738 \\ \mathrm{H} & -2.935340 & -2.719831 & 4.832549 \\ \mathrm{H} & -5.432524 & 0.229726 & 2.951599 \\ \mathrm{C} & -5.547352 & -2.170240 & 4.267493 \\ \mathrm{H} & -5.443512 & -2.649496 & 5.247038 \\ \mathrm{H} & -6.456068 & -1.559281 & 4.282872 \\ \mathrm{H} & -5.694555 & -2.967547 & 3.529038 \\ \mathrm{O} & 0.351069 & 0.936756 & 3.773119 \\ \mathrm{O} & -1.042272 & 2.308937 & 2.153668 \\ \mathrm{~N} & 0.034777 & 0.190027 & 1.319165\end{array}$

\section{Structure: ${ }^{3}$ TS12}

$\begin{array}{lrrr}\mathrm{C} & 1.740877 & 2.910352 & -0.843412 \\ \mathrm{C} & 1.785322 & 4.324263 & -0.964226 \\ \mathrm{C} & 0.520398 & 4.804981 & -0.741428 \\ \mathrm{C} & -0.332919 & 3.700430 & -0.475793 \\ \mathrm{~N} & 0.438863 & 2.520443 & -0.479760 \\ \mathrm{H} & 2.657517 & 4.893503 & -1.250913 \\ \mathrm{H} & 0.192247 & 5.830997 & -0.822005 \\ \mathrm{C} & -3.431084 & 0.669413 & -0.731914 \\ \mathrm{C} & -4.546603 & 1.554587 & -0.495959 \\ \mathrm{C} & -4.028179 & 2.796547 & -0.307399 \\ \mathrm{C} & -2.593549 & 2.681405 & -0.432509 \\ \mathrm{~N} & -2.253374 & 1.370105 & -0.665523 \\ \mathrm{H} & -5.585338 & 1.260832 & -0.462584 \\ \mathrm{H} & -4.564265 & 3.707820 & -0.088796 \\ \mathrm{C} & 1.836321 & -1.374555 & -1.556318 \\ \mathrm{C} & 3.264015 & -1.470795 & -1.750239\end{array}$




\begin{tabular}{|c|c|c|c|}
\hline $\mathrm{C}$ & 3.772459 & -0.227509 & -1.563730 \\
\hline C & 2.659093 & 0.648647 & -1.281556 \\
\hline $\mathrm{N}$ & 1.489806 & -0.077165 & -1.267734 \\
\hline $\mathrm{H}$ & 3.806756 & -2.370316 & -1.996454 \\
\hline $\mathrm{H}$ & 4.808224 & 0.069390 & -1.616633 \\
\hline C & -0.432916 & -2.360026 & -1.489404 \\
\hline C & -1.342387 & -3.475990 & -1.584195 \\
\hline C & -2.597419 & -2.981495 & -1.428654 \\
\hline $\mathrm{C}$ & -2.471330 & -1.563294 & -1.205908 \\
\hline $\mathrm{N}$ & -1.141259 & -1.208014 & -1.228524 \\
\hline $\mathrm{H}$ & -1.060812 & -4.509606 & -1.703791 \\
\hline $\mathrm{H}$ & -3.525842 & -3.532734 & -1.449756 \\
\hline C & 2.793508 & 2.035699 & -1.103486 \\
\hline C & -3.547151 & -0.701999 & -0.974924 \\
\hline C & -1.719026 & 3.776079 & -0.368548 \\
\hline C & 0.955156 & -2.457770 & -1.649999 \\
\hline $\mathrm{Mn}$ & -0.413640 & 0.615676 & -1.018550 \\
\hline $\mathrm{Cl}$ & -0.552079 & 1.137778 & -3.177778 \\
\hline $\mathrm{C}$ & 1.524619 & -3.800718 & -1.963921 \\
\hline $\mathrm{C}$ & 2.427929 & -4.444129 & -1.110814 \\
\hline C & 1.175339 & -4.482057 & -3.136785 \\
\hline $\mathrm{C}$ & 2.945606 & -5.705378 & -1.388041 \\
\hline $\mathrm{C}$ & 1.672945 & -5.748639 & -3.433697 \\
\hline $\mathrm{C}$ & 2.561682 & -6.364122 & -2.553897 \\
\hline C & 4.144619 & 2.648754 & -1.225561 \\
\hline C & 4.704032 & 3.345230 & -0.147113 \\
\hline C & 4.909473 & 2.571900 & -2.395288 \\
\hline C & 5.983079 & 3.889264 & -0.197404 \\
\hline $\mathrm{C}$ & 6.190305 & 3.115152 & -2.473636 \\
\hline $\mathrm{C}$ & 6.729296 & 3.774152 & -1.369542 \\
\hline $\mathrm{C}$ & -2.308093 & 5.137449 & -0.232648 \\
\hline C & -3.134524 & 5.678009 & -1.225232 \\
\hline $\mathrm{C}$ & -2.039004 & 5.937027 & 0.885986 \\
\hline C & -3.691859 & 6.948500 & -1.108675 \\
\hline $\mathrm{C}$ & -2.583301 & 7.213470 & 1.018878 \\
\hline C & -3.413151 & 7.718189 & 0.019574 \\
\hline $\mathrm{C}$ & -4.915988 & -1.291979 & -0.951397 \\
\hline C & -5.860923 & -1.036689 & -1.949011 \\
\hline $\mathrm{C}$ & -5.308881 & -2.121758 & 0.099937 \\
\hline $\mathrm{C}$ & -7.143704 & -1.582582 & -1.900837 \\
\hline $\mathrm{C}$ & -6.578402 & -2.683359 & 0.167962 \\
\hline $\mathrm{C}$ & -7.503416 & -2.411808 & -0.838318 \\
\hline $\mathrm{F}$ & 2.845809 & -3.838316 & 0.011130 \\
\hline $\mathrm{F}$ & 3.806079 & -6.281432 & -0.541730 \\
\hline
\end{tabular}




\begin{tabular}{|c|c|c|c|}
\hline$F$ & 3.048199 & -7.574495 & -2.831347 \\
\hline $\mathrm{F}$ & 1.311465 & -6.371260 & -4.559416 \\
\hline $\mathrm{F}$ & 0.330476 & -3.919551 & -4.009984 \\
\hline $\mathrm{F}$ & -5.547319 & -0.242275 & -2.977095 \\
\hline F & -8.028475 & -1.320453 & -2.866505 \\
\hline $\mathrm{F}$ & -8.725719 & -2.942268 & -0.786599 \\
\hline $\mathrm{F}$ & -6.906727 & -3.483048 & 1.19535 \\
\hline $\mathrm{F}$ & -4.438589 & -2.408605 & 1.077733 \\
\hline F & -3.411060 & 4.966447 & -2.325514 \\
\hline $\mathrm{F}$ & -4.480045 & 7.436581 & -2.072024 \\
\hline $\mathrm{F}$ & -3.937727 & 8.938966 & 0.140033 \\
\hline F & -2.314451 & 7.955765 & 2.097237 \\
\hline $\mathrm{F}$ & -1.236830 & 5.492090 & 1.85415 \\
\hline $\mathrm{F}$ & 4.007824 & 3.480388 & 0.992855 \\
\hline F & 6.497688 & 4.511227 & 0.867015 \\
\hline F & 7.955415 & 4.293470 & -1.43422 \\
\hline F & 6.899684 & 3.016402 & -3.601214 \\
\hline $\mathrm{F}$ & 4.421658 & 1.952620 & -3.476518 \\
\hline C & -0.910459 & -3.532976 & 1.957672 \\
\hline $\mathrm{C}$ & -0.607919 & -2.221249 & 1.687955 \\
\hline C & 0.712157 & -1.730099 & 1.891401 \\
\hline $\mathrm{C}$ & 1.721661 & -2.635949 & 2.366479 \\
\hline C & 1.349899 & -3.988177 & 2.613228 \\
\hline C & 0.067445 & -4.435152 & 2.423416 \\
\hline $\mathrm{H}$ & -1.383712 & -1.558026 & 1.334874 \\
\hline $\mathrm{C}$ & 1.075369 & -0.360894 & 1.661515 \\
\hline C & 3.066961 & -2.195451 & 2.567289 \\
\hline $\mathrm{H}$ & 2.093946 & -4.693610 & 2.961244 \\
\hline $\mathrm{H}$ & -0.199335 & -5.466607 & 2.62061 \\
\hline $\mathrm{C}$ & 3.383609 & -0.867313 & 2.306018 \\
\hline $\mathrm{C}$ & 2.381016 & 0.031933 & 1.875337 \\
\hline 0 & -2.244579 & -3.946742 & 1.877012 \\
\hline $\mathrm{C}$ & -3.968950 & -5.517024 & 1.498632 \\
\hline $\mathrm{H}$ & -4.016927 & -5.907148 & 2.522194 \\
\hline $\mathrm{H}$ & -4.656962 & -4.673328 & 1.442702 \\
\hline $\mathrm{H}$ & -4.261015 & -6.304860 & 0.802992 \\
\hline C & -2.550037 & -5.096187 & 1.206084 \\
\hline 0 & -1.773422 & -5.700481 & 0.502484 \\
\hline $\mathrm{C}$ & 4.797054 & -0.404203 & 2.565889 \\
\hline C & 5.830394 & -1.517142 & 2.289629 \\
\hline $\mathrm{C}$ & 5.377195 & 0.836428 & 1.866202 \\
\hline $\mathrm{H}$ & 4.948066 & 1.767683 & 2.237010 \\
\hline $\mathrm{C}$ & 6.889988 & 0.724091 & 2.166981 \\
\hline $\mathrm{H}$ & 7.174240 & 1.308523 & 3.050569 \\
\hline
\end{tabular}




$\begin{array}{lrrr}\mathrm{C} & 7.145209 & -0.763223 & 2.487244 \\ \mathrm{O} & 8.204580 & -1.242232 & 2.827998 \\ \mathrm{C} & 5.845035 & -2.002276 & 0.817607 \\ \mathrm{H} & 6.598939 & -2.789010 & 0.707264 \\ \mathrm{H} & 4.871899 & -2.397853 & 0.517819 \\ \mathrm{H} & 6.100983 & -1.198071 & 0.120209 \\ \mathrm{H} & 7.540973 & 1.057062 & 1.351189 \\ \mathrm{H} & 5.182345 & 0.790664 & 0.790074 \\ \mathrm{C} & 5.544486 & -2.672101 & 3.241576 \\ \mathrm{C} & 4.118264 & -3.217392 & 2.996296 \\ \mathrm{H} & 5.636645 & -2.309375 & 4.272808 \\ \mathrm{H} & 6.280065 & -3.475430 & 3.125005 \\ \mathrm{H} & 3.782810 & -3.731215 & 3.905593 \\ \mathrm{H} & 4.156721 & -3.992125 & 2.218213 \\ \mathrm{H} & 2.635083 & 1.075427 & 1.721104 \\ \mathrm{H} & 0.429777 & 1.822304 & 0.504407 \\ \mathrm{H} & 4.864845 & -0.195389 & 3.647916 \\ \mathrm{~S} & -0.659981 & 1.340634 & 2.631583 \\ \mathrm{C} & -2.043962 & 0.328796 & 3.146920 \\ \mathrm{C} & -1.841756 & -0.715667 & 4.048509 \\ \mathrm{C} & -3.313528 & 0.606247 & 2.638832 \\ \mathrm{C} & -2.923527 & -1.521212 & 4.402317 \\ \mathrm{H} & -0.851850 & -0.897386 & 4.451181 \\ \mathrm{C} & -4.382339 & -0.200252 & 3.013468 \\ \mathrm{H} & -3.455603 & 1.450876 & 1.977956 \\ \mathrm{C} & -4.202661 & -1.287104 & 3.882682 \\ \mathrm{H} & -2.767306 & -2.351283 & 5.086021 \\ \mathrm{H} & -5.372547 & 0.008270 & 2.613950 \\ \mathrm{C} & -5.369032 & -2.180489 & 4.228290 \\ \mathrm{H} & -6.074516 & -2.982915 & 4.911565 \\ \mathrm{H} & -5.784162 & -2.640776 & 3.324397 \\ \mathrm{H} & -1.3054 & 1.335123 & 3.741606 \\ \mathrm{O} & 2.612761 & 2.147127 \\ \mathrm{O} & 0.620543 & 1.265467\end{array}$

\section{Structure: ${ }^{5}$ TS12}

$\begin{array}{lrrr}\mathrm{C} & 1.703338 & 2.963391 & -0.838223 \\ \mathrm{C} & 1.721521 & 4.371084 & -1.019255 \\ \mathrm{C} & 0.447883 & 4.837303 & -0.800560 \\ \mathrm{C} & -0.378372 & 3.728046 & -0.476919 \\ \mathrm{~N} & 0.418430 & 2.573602 & -0.441601 \\ \mathrm{H} & 2.576373 & 4.940251 & -1.354580\end{array}$




\begin{tabular}{|c|c|c|c|}
\hline $\mathrm{H}$ & 0.094333 & 5.849567 & -0.932298 \\
\hline $\mathrm{C}$ & -3.429098 & 0.603641 & -0.773395 \\
\hline $\mathrm{C}$ & -4.539685 & 1.465059 & -0.457829 \\
\hline $\mathrm{C}$ & -4.035341 & 2.710387 & -0.251734 \\
\hline C & -2.610330 & 2.638259 & -0.448082 \\
\hline $\mathrm{N}$ & -2.263756 & 1.335193 & -0.751262 \\
\hline $\mathrm{H}$ & -5.568724 & 1.149380 & -0.372769 \\
\hline $\mathrm{H}$ & -4.576337 & 3.599412 & 0.034569 \\
\hline $\mathrm{C}$ & 1.801920 & -1.351755 & -1.605372 \\
\hline $\mathrm{C}$ & 3.232061 & -1.423558 & -1.759829 \\
\hline $\mathrm{C}$ & 3.724081 & -0.176240 & -1.553184 \\
\hline C & 2.605389 & 0.694862 & -1.303207 \\
\hline $\mathrm{N}$ & 1.437653 & -0.048678 & -1.337861 \\
\hline $\mathrm{H}$ & 3.793077 & -2.316804 & -1.984436 \\
\hline $\mathrm{H}$ & 4.759378 & 0.125242 & -1.562624 \\
\hline $\mathrm{C}$ & -0.435150 & -2.413017 & -1.526381 \\
\hline $\mathrm{C}$ & -1.327016 & -3.547992 & -1.574849 \\
\hline C & -2.589344 & -3.068124 & -1.424361 \\
\hline C & -2.483348 & -1.640006 & -1.248125 \\
\hline $\mathrm{N}$ & -1.163084 & -1.271102 & -1.306817 \\
\hline $\mathrm{H}$ & -1.029248 & -4.580652 & -1.660006 \\
\hline $\mathrm{H}$ & -3.508021 & -3.635581 & -1.413073 \\
\hline $\mathrm{C}$ & 2.747476 & 2.079254 & -1.107958 \\
\hline $\mathrm{C}$ & -3.552373 & -0.770584 & -1.017168 \\
\hline C & -1.767670 & 3.758081 & -0.369189 \\
\hline $\mathrm{C}$ & 0.953746 & -2.465659 & -1.693852 \\
\hline $\mathrm{Mn}$ & -0.449329 & 0.623847 & -1.181139 \\
\hline $\mathrm{Cl}$ & -0.468800 & 1.496560 & -3.346819 \\
\hline $\mathrm{C}$ & 1.557933 & -3.796053 & -1.994534 \\
\hline $\mathrm{C}$ & 2.480479 & -4.412682 & -1.142050 \\
\hline C & 1.206456 & -4.500148 & -3.153743 \\
\hline C & 3.018069 & -5.667797 & -1.408866 \\
\hline $\mathrm{C}$ & 1.724371 & -5.760961 & -3.439957 \\
\hline C & 2.633905 & -6.348480 & -2.562084 \\
\hline $\mathrm{C}$ & 4.100184 & 2.687057 & -1.226067 \\
\hline C & 4.652466 & 3.385495 & -0.144570 \\
\hline C & 4.870051 & 2.609184 & -2.392981 \\
\hline C & 5.931061 & 3.930992 & -0.190105 \\
\hline $\mathrm{C}$ & 6.149556 & 3.155887 & -2.465959 \\
\hline $\mathrm{C}$ & 6.681855 & 3.816952 & -1.359515 \\
\hline C & -2.392042 & 5.099079 & -0.211243 \\
\hline C & -3.282835 & 5.612377 & -1.163147 \\
\hline C & -2.087414 & 5.912093 & 0.890061 \\
\hline C & -3.868354 & 6.867325 & -1.021675 \\
\hline
\end{tabular}


C

C

$\mathrm{H}$

$\mathrm{H}$

C

C

O

C

$\mathrm{H}$

\begin{tabular}{|c|c|c|}
\hline-2.660869 & 7.172827 & 1.046383 \\
\hline-3.554482 & 7.649283 & 0.089010 \\
\hline-4.923109 & -1.353759 & -0.969504 \\
\hline & -1.082444 & -1 \\
\hline 5.301766 & -2.187773 & \\
\hline 7.171917 & -1.617517 & -1.875 \\
\hline 0 & -2.73 & \\
\hline & -2.4497 & \\
\hline & -3.7857 & -0.03 \\
\hline 3. & -6.21 & -0.5 \\
\hline & -7.55 & -2.8 \\
\hline & -6.40 & -4.5 \\
\hline & -3.96 & \\
\hline 5 & -0.2 & -2.9 \\
\hline 8 & $-1 \cdot 3$ & -2.8 \\
\hline 8 . & -2.9 & -0 . \\
\hline 6. & -3.5 & 1.2 \\
\hline-4 & -2.4 & \\
\hline 3. & & -2 \\
\hline-4 . & 7.3 & -1 \\
\hline-4 . & $8 . \varepsilon$ & \\
\hline-2 . & 7. & \\
\hline-1.2 & & \\
\hline 3. & 3.5 & \\
\hline 6 . & 4.5 & \\
\hline & & \\
\hline 6 . & 3.0 & -3.5 \\
\hline 4. & 1.9 & -3 \\
\hline-0 . & -3.5 & \\
\hline-0 . & -2.2 & \\
\hline 0 & $-1 \cdot 7$ & \\
\hline & -2.6 & \\
\hline & -3.9 & \\
\hline 0.1 & -4.4 & \\
\hline$-1 \cdot 3$ & -1.53 & \\
\hline 1.0 & -0.3 & \\
\hline 3.10 & -2.1 & 2.5 \\
\hline 2.15 & -4.697297 & 2.862 \\
\hline-0.13 & -5.4 & \\
\hline 3.40 & -0.84 & 2.3 \\
\hline 2.38 & 0.0 & 1.9 \\
\hline-2.211862 & -3.942940 & 1.9 \\
\hline$-3 \cdot 9$ & -5.5 & \\
\hline-3.9 & -5.8 & 2.7 \\
\hline
\end{tabular}




\begin{tabular}{|c|c|c|c|}
\hline $\mathrm{H}$ & -4.652250 & -4.720599 & 1.531523 \\
\hline $\mathrm{H}$ & -4.223306 & -6.396102 & 1.053278 \\
\hline $\mathrm{C}$ & -2.541139 & -5.103764 & 1.282022 \\
\hline O & -1.800007 & -5.709917 & 0.541571 \\
\hline C & 4.816062 & -0.367689 & 2.560731 \\
\hline C & 5.860679 & -1.462633 & 2.257110 \\
\hline $\mathrm{C}$ & 5.377925 & 0.892722 & 1.881055 \\
\hline $\mathrm{H}$ & 4.937758 & 1.812747 & 2.267500 \\
\hline $\mathrm{C}$ & 6.892855 & 0.793336 & 2.175422 \\
\hline $\mathrm{H}$ & 7.171401 & 1.362444 & 3.070750 \\
\hline C & 7.167585 & -0.697394 & 2.464086 \\
\hline O & 8.234741 & -1.169969 & 2.789205 \\
\hline $\mathrm{C}$ & 5.874969 & -1.916526 & 0.775136 \\
\hline $\mathrm{H}$ & 6.635221 & -2.694097 & 0.645314 \\
\hline $\mathrm{H}$ & 4.903886 & -2.314530 & 0.472138 \\
\hline $\mathrm{H}$ & 6.122944 & -1.095442 & 0.094356 \\
\hline $\mathrm{H}$ & 7.538134 & 1.151688 & 1.365819 \\
\hline $\mathrm{H}$ & 5.181097 & 0.862649 & 0.804640 \\
\hline C & 5.591191 & -2.641514 & 3.184488 \\
\hline $\mathrm{C}$ & 4.170889 & -3.199198 & 2.930910 \\
\hline $\mathrm{H}$ & 5.680994 & -2.299272 & 4.222919 \\
\hline $\mathrm{H}$ & 6.336904 & -3.432649 & 3.049557 \\
\hline $\mathrm{H}$ & 3.846549 & -3.738924 & 3.829265 \\
\hline $\mathrm{H}$ & 4.216709 & -3.955542 & 2.135268 \\
\hline $\mathrm{H}$ & 2.630025 & 1.112271 & 1.789226 \\
\hline $\mathrm{H}$ & 0.382852 & 1.895971 & 0.476775 \\
\hline $\mathrm{H}$ & 4.889801 & -0.177419 & 3.645835 \\
\hline S & -0.620851 & 1.379947 & 2.687719 \\
\hline $\mathrm{C}$ & -1.953886 & 0.322732 & 3.254734 \\
\hline $\mathrm{C}$ & -1.697037 & -0.694636 & 4.173004 \\
\hline C & -3.225376 & 0.486075 & 2.704145 \\
\hline C & -2.717841 & -1.587828 & 4.497491 \\
\hline $\mathrm{H}$ & -0.706373 & -0.794714 & 4.602523 \\
\hline C & -4.235105 & -0.405935 & 3.049221 \\
\hline $\mathrm{H}$ & -3.410736 & 1.305816 & 2.021858 \\
\hline $\mathrm{C}$ & -3.992641 & -1.471210 & 3.929246 \\
\hline $\mathrm{H}$ & -2.513487 & -2.401553 & 5.188633 \\
\hline $\mathrm{H}$ & -5.224252 & -0.287720 & 2.611948 \\
\hline $\mathrm{C}$ & -5.087197 & -2.460196 & 4.250661 \\
\hline $\mathrm{H}$ & -4.677551 & -3.384137 & 4.671652 \\
\hline $\mathrm{H}$ & -5.795105 & -2.048594 & 4.982013 \\
\hline $\mathrm{H}$ & -5.657667 & -2.722059 & 3.353310 \\
\hline O & 0.341308 & 1.476422 & 3.797359 \\
\hline O & -1.254132 & 2.606047 & 2.165389 \\
\hline
\end{tabular}




\section{Structure: ${ }^{3}$ TS2b-m1}

\begin{tabular}{|c|c|c|c|}
\hline $\mathrm{C}$ & -1.831277 & -2.645985 & -0.475192 \\
\hline C & -1.593403 & -4.071646 & -0.481207 \\
\hline C & -0.265671 & -4.255358 & -0.253505 \\
\hline $\mathrm{C}$ & 0.333655 & -2.943572 & -0.173170 \\
\hline $\mathrm{N}$ & -0.637873 & -1.991695 & -0.312836 \\
\hline $\mathrm{H}$ & -2.351359 & -4.828254 & -0.619701 \\
\hline $\mathrm{H}$ & 0.275834 & -5.188340 & -0.18887 \\
\hline C & 2.560225 & 0.672862 & -0.83678 \\
\hline $\mathrm{C}$ & 3.867306 & 0.064166 & -0.870862 \\
\hline $\mathrm{C}$ & 3.711917 & -1.239396 & -0.51995 \\
\hline $\mathrm{C}$ & 2.299544 & -1.461770 & -0.328874 \\
\hline $\mathrm{N}$ & 1.626163 & -0.280443 & -0.518976 \\
\hline $\mathrm{H}$ & 4.789381 & 0.586383 & -1.07930 \\
\hline $\mathrm{H}$ & 4.482743 & -1.984302 & -0.397076 \\
\hline C & -2.947038 & 1.441146 & 0.261870 \\
\hline C & -4.366042 & 1.204555 & 0.39367 \\
\hline $\mathrm{C}$ & -4.586065 & -0.091541 & 0.04895 \\
\hline $\mathrm{C}$ & -3.297886 & -0.678563 & -0.24061 \\
\hline $\mathrm{N}$ & -2.326537 & 0.276074 & -0.10748 \\
\hline $\mathrm{H}$ & -5.096679 & 1.942110 & 0.69076 \\
\hline $\mathrm{H}$ & -5.527196 & -0.621570 & 0.02692 \\
\hline $\mathrm{C}$ & -0.985771 & 2.924216 & 0.04567 \\
\hline $\mathrm{C}$ & -0.413450 & 4.235970 & -0.13014 \\
\hline $\mathrm{C}$ & 0.853225 & 4.062043 & -0.59589 \\
\hline $\mathrm{C}$ & 1.090460 & 2.641840 & -0.66034 \\
\hline $\mathrm{N}$ & -0.047772 & 1.975352 & -0.27722 \\
\hline $\mathrm{H}$ & -0.934374 & 5.167046 & 0.03916 \\
\hline $\mathrm{H}$ & 1.568464 & 4.825454 & -0.86450 \\
\hline C & -3.090521 & -2.040154 & -0.48913 \\
\hline $\mathrm{C}$ & 2.318861 & 2.043410 & -0.95611 \\
\hline C & 1.708815 & -2.706913 & -0.09668 \\
\hline $\mathrm{C}$ & -2.324276 & 2.687159 & 0.377763 \\
\hline Mn & -0.337557 & -0.002115 & -0.27160 \\
\hline $\mathrm{Cl}$ & -0.603176 & 0.036043 & -2.58989 \\
\hline O & -0.227606 & -0.108250 & 1.45528 \\
\hline $\mathrm{C}$ & 5.126764 & -1.247219 & 2.88214 \\
\hline $\mathrm{C}$ & 3.885237 & -1.787827 & 3.23871 \\
\hline $\mathrm{C}$ & 2.737275 & -1.004579 & 3.16923 \\
\hline C & 2.806069 & 0.336749 & 2.73881 \\
\hline $\mathrm{C}$ & 4.056424 & 0.856944 & 2.3538 \\
\hline
\end{tabular}




\begin{tabular}{|c|c|c|c|}
\hline C & 5.207419 & 0.076220 & 2.440998 \\
\hline $\mathrm{H}$ & 6.024163 & -1.856930 & 2.945659 \\
\hline $\mathrm{H}$ & 3.811961 & -2.818500 & 3.572349 \\
\hline $\mathrm{H}$ & 1.770804 & -1.430104 & 3.421912 \\
\hline $\mathrm{H}$ & 4.116889 & 1.883634 & 2.008237 \\
\hline $\mathrm{H}$ & 6.168263 & 0.498505 & 2.158868 \\
\hline C & 1.589939 & 1.166217 & 2.691729 \\
\hline $\mathrm{H}$ & 0.757382 & 0.599231 & 1.967173 \\
\hline $\mathrm{H}$ & 0.997863 & 1.172367 & 3.611681 \\
\hline $\mathrm{H}$ & 1.722611 & 2.168210 & 2.279365 \\
\hline C & -3.139941 & 3.859125 & 0.802214 \\
\hline C & -2.884304 & 4.497201 & 2.020763 \\
\hline $\mathrm{C}$ & -4.161491 & 4.384995 & 0.004845 \\
\hline C & -3.616247 & 5.605691 & 2.439035 \\
\hline C & -4.912322 & 5.488054 & 0.405848 \\
\hline $\mathrm{C}$ & -4.635678 & 6.100378 & 1.627126 \\
\hline $\mathrm{C}$ & 3.471412 & 2.919164 & -1.304512 \\
\hline C & 4.047574 & 2.884367 & -2.578810 \\
\hline C & 4.041351 & 3.788584 & -0.369992 \\
\hline $\mathrm{C}$ & 5.141556 & 3.681828 & -2.911114 \\
\hline $\mathrm{C}$ & 5.131966 & 4.596530 & -0.677152 \\
\hline C & 5.682185 & 4.542291 & -1.957115 \\
\hline $\mathrm{C}$ & 2.596364 & -3.859578 & 0.219175 \\
\hline $\mathrm{C}$ & 2.611705 & -4.395007 & 1.510306 \\
\hline $\mathrm{C}$ & 3.460041 & -4.430510 & -0.719859 \\
\hline $\mathrm{C}$ & 3.476254 & -5.423449 & 1.872008 \\
\hline $\mathrm{C}$ & 4.324958 & -5.470873 & -0.385599 \\
\hline C & 4.333816 & -5.966772 & 0.917401 \\
\hline $\mathrm{C}$ & -4.295303 & -2.895732 & -0.679827 \\
\hline $\mathrm{C}$ & -5.083191 & -2.771131 & -1.829541 \\
\hline C & -4.702449 & -3.828229 & 0.279491 \\
\hline C & -6.223608 & -3.547674 & -2.025286 \\
\hline $\mathrm{C}$ & -5.834365 & -4.619973 & 0.102017 \\
\hline $\mathrm{C}$ & -6.597734 & -4.476081 & -1.055351 \\
\hline $\mathrm{F}$ & -6.197288 & -5.506523 & 1.035188 \\
\hline $\mathrm{F}$ & -3.994195 & -3.980670 & 1.407085 \\
\hline $\mathrm{F}$ & -7.686614 & -5.226647 & -1.233487 \\
\hline $\mathrm{F}$ & -6.957311 & -3.410270 & -3.133789 \\
\hline $\mathrm{F}$ & -4.750283 & -1.885231 & -2.773136 \\
\hline $\mathrm{F}$ & -1.907239 & 4.044648 & 2.817862 \\
\hline $\mathrm{F}$ & -4.442015 & 3.824816 & -1.178136 \\
\hline $\mathrm{F}$ & -5.885916 & 5.967483 & -0.374538 \\
\hline $\mathrm{F}$ & -5.345855 & 7.159903 & 2.018878 \\
\hline & -3.351083 & 6.193942 & 3.609928 \\
\hline
\end{tabular}




$\begin{array}{lrrr}F & 3.540751 & 3.853934 & 0.878634 \\ F & 3.554143 & 2.064872 & -3.511220 \\ F & 5.671919 & 3.630305 & -4.135952 \\ F & 6.728176 & 5.310525 & -2.266139 \\ F & 5.655560 & 5.413507 & 0.242621 \\ F & 1.798911 & -3.898053 & 2.454393 \\ F & 3.484855 & -3.963693 & -1.974238 \\ F & 5.143937 & -5.994935 & -1.302870 \\ F & 5.166120 & -6.955042 & 1.250834 \\ F & 3.499366 & -5.873633 & 3.131734\end{array}$

\section{Structure: ${ }^{5} \mathrm{TS} 2 \mathrm{~b}-$ model1}

\begin{tabular}{|c|c|c|c|}
\hline C & -1.872970 & -2.626674 & -0.453987 \\
\hline C & -1.648071 & -4.052704 & -0.44284 \\
\hline C & -0.322740 & -4.247430 & -0.20842 \\
\hline C & 0.292668 & -2.944528 & -0.14283 \\
\hline N & -0.673435 & -1.978556 & -0.2947 \\
\hline $\mathrm{H}$ & -2.412692 & -4.803553 & -0.5757 \\
\hline $\mathrm{H}$ & 0.207139 & -5.185966 & -0.13058 \\
\hline C & 2.566836 & 0.634220 & -0.82278 \\
\hline C & 3.868093 & 0.009711 & -0.8543 \\
\hline C & 3.695874 & -1.291320 & -0.50325 \\
\hline C & 2.279452 & -1.491370 & -0.31081 \\
\hline $\mathrm{N}$ & 1.621084 & -0.307328 & -0.5075 \\
\hline $\mathrm{H}$ & 4.797197 & 0.520837 & -1.05865 \\
\hline $\mathrm{H}$ & 4.456357 & -2.046088 & -0.37632 \\
\hline $\mathrm{C}$ & -2.929392 & 1.473351 & 0.2594 \\
\hline C & -4.350097 & 1.255930 & 0.3931 \\
\hline C & -4.590674 & -0.036401 & 0.04806 \\
\hline C & -3.312946 & -0.643729 & -0.24113 \\
\hline $\mathrm{N}$ & -2.323948 & 0.293545 & $-0.1101^{\prime}$ \\
\hline $\mathrm{H}$ & -5.069325 & 2.003559 & 0.6926 \\
\hline $\mathrm{H}$ & -5.539913 & -0.551830 & 0.0269 \\
\hline $\mathrm{C}$ & -0.949878 & 2.933424 & 0.04535 \\
\hline C & -0.363734 & 4.239445 & -0.13092 \\
\hline C & 0.902854 & 4.048442 & -0.59063 \\
\hline $\mathrm{C}$ & 1.120679 & 2.623991 & -0.65098 \\
\hline $\mathrm{N}$ & -0.027156 & 1.974135 & -0.27245 \\
\hline $\mathrm{H}$ & -0.874137 & 5.176951 & 0.03476 \\
\hline $\mathrm{H}$ & 1.628489 & 4.802294 & $-0.8580 s$ \\
\hline C & -3.125076 & -2.008817 & $-0.4830 s$ \\
\hline & 2.342437 & 2.008014 & -0.94269 \\
\hline
\end{tabular}


C

C

Mn

$\mathrm{Cl}$

O

C

C

C

C

C

C

$\mathrm{H}$

$\mathrm{H}$

$\mathrm{H}$

$\mathrm{H}$

$\mathrm{H}$

C

$\mathrm{H}$

$\mathrm{H}$

$\mathrm{H}$

C

C

C

C

C

C

C

C

C

C

C

C

C

C

C

C

C

C

C

C

C

C

C

C

\begin{tabular}{|c|c|c|}
\hline 1.668445 & -2.728138 & -0.071045 \\
\hline-2.294043 & 2.709925 & 0.375080 \\
\hline-0.357911 & -0.014405 & -0.290584 \\
\hline 0.54234 & 0.090586 & -2.604530 \\
\hline 0.21882 & -0.100358 & 1.476049 \\
\hline 5.14945 & -1.260147 & \\
\hline & -1.79 & 3.1 \\
\hline & $-0.99^{\circ}$ & \\
\hline 2 & 0.35 & \\
\hline & 0.86 & 2.3 \\
\hline & 0.0 & \\
\hline 04 & $-1.8 \varepsilon$ & \\
\hline & -2.8 & \\
\hline 1.7 & -1.4 & \\
\hline 4.1 & 1.9 & \\
\hline 6 & 0.4 & \\
\hline 1.6 & 1.1 & 2.6 \\
\hline 0.8 & 0.6 & \\
\hline & & \\
\hline 1.7 & 2.2 & 2.2 \\
\hline-3.0 & 3.8 & \\
\hline-2.8 & & \\
\hline-4.11 & 4.42 & -0.00 \\
\hline-3.5 & 5.6 & 2.4 \\
\hline-4.84 & 5.5 & \\
\hline-4.5 & & \\
\hline 3.5 & 2.8 & -1.2 \\
\hline 4.0 & 2.8 & -2.5 \\
\hline & 3.7 & -0.3 \\
\hline 5.1 & 3.5 & -2.9 \\
\hline 5.1$\}$ & 4.53 & -0.6 \\
\hline 5.73 & 4.4 & -1 \\
\hline 2.54 & -3.8 & 0.2 \\
\hline 2.5 & -4.43 & 1.5 \\
\hline 3.38 & -4.47 & -0.713368 \\
\hline 3.4 & -5.47 & \\
\hline 4.233 & -5.53408 & -0.3916 \\
\hline 4.2500 & -6.03227 & 0.91048 \\
\hline-4.339593 & -2.849726 & -0.673595 \\
\hline-5.123378 & -2.718091 & -1.825402 \\
\hline-4.761652 & -3.773664 & 0.28767 \\
\hline-6.273681 & -3.479710 & -2.02152 \\
\hline & -4.550849 & 0.1097 \\
\hline-6.66 & -4.4 & -1.049 \\
\hline
\end{tabular}




$\begin{array}{lrrr}\text { F } & -6.280227 & -5.429702 & 1.044648 \\ F & -4.058068 & -3.932248 & 1.417322 \\ F & -7.760687 & -5.136633 & -1.228175 \\ F & -7.002799 & -3.335880 & -3.132150 \\ F & -4.776300 & -1.839496 & -2.770985 \\ F & -1.863475 & 4.065394 & 2.812962 \\ F & -4.397311 & 3.870278 & -1.184735 \\ F & -5.813986 & 6.033145 & -0.386881 \\ F & -5.260862 & 7.223147 & 2.004769 \\ F & -3.280608 & 6.234580 & 3.599799 \\ F & 3.577696 & 3.836491 & 0.871923 \\ F & 3.586290 & 1.981418 & -3.491325 \\ F & 5.722623 & 3.514094 & -4.131306 \\ F & 6.791733 & 5.209363 & -2.282173 \\ F & 5.711662 & 5.360394 & 0.220922 \\ F & 1.773704 & -3.919461 & 2.483142 \\ F & 3.398149 & -4.010914 & -1.967493 \\ F & 5.031585 & -6.071112 & -1.319880 \\ F & 5.068443 & -7.035934 & 1.231798 \\ F & 3.445022 & -5.929371 & 3.134834\end{array}$

\section{Structure: ${ }^{3} \mathrm{TS} 4-\mathrm{m} 1$}

$\begin{array}{lrrr}\text { C } & -2.186149 & -2.199376 & -0.556272 \\ \mathrm{C} & -2.326764 & -3.638637 & -0.501046 \\ \mathrm{C} & -1.095284 & -4.148792 & -0.244745 \\ \mathrm{C} & -0.176136 & -3.032215 & -0.207300 \\ \mathrm{~N} & -0.868268 & -1.866939 & -0.395839 \\ \mathrm{H} & -3.253705 & -4.179443 & -0.621411 \\ \mathrm{H} & -0.823684 & -5.186578 & -0.120924 \\ \mathrm{C} & 2.918175 & -0.082809 & -0.761924 \\ \mathrm{C} & 4.032412 & -0.996964 & -0.717259 \\ \mathrm{C} & 3.530663 & -2.223661 & -0.406548 \\ \mathrm{C} & 2.099505 & -2.079532 & -0.290981 \\ \mathrm{~N} & 1.757938 & -0.766559 & -0.498211 \\ \mathrm{H} & 5.064507 & -0.726234 & -0.886700 \\ \mathrm{H} & 4.071957 & -3.152259 & -0.297009 \\ \mathrm{C} & -2.281557 & 2.076555 & -0.053926 \\ \mathrm{C} & -3.720020 & 2.202360 & 0.032927 \\ \mathrm{C} & -4.241102 & 0.980866 & -0.249991 \\ \mathrm{C} & -3.125871 & 0.082306 & -0.457256 \\ \mathrm{~N} & -1.955041 & 0.777300 & -0.337565 \\ \mathrm{H} & -4.255452 & 3.112222 & 0.260286\end{array}$




\begin{tabular}{|c|c|c|c|}
\hline $\mathrm{H}$ & -5.282472 & 0.695345 & -0.283512 \\
\hline C & -0.007138 & 3.018540 & -0.237302 \\
\hline $\mathrm{C}$ & 0.882084 & 4.137906 & -0.432939 \\
\hline $\mathrm{C}$ & 2.091147 & 3.630828 & -0.795152 \\
\hline $\mathrm{C}$ & 1.969445 & 2.194217 & -0.779657 \\
\hline $\mathrm{N}$ & 0.678973 & 1.850678 & -0.452327 \\
\hline $\mathrm{H}$ & 0.599745 & 5.176829 & -0.343024 \\
\hline $\mathrm{H}$ & 2.990158 & 4.175664 & -1.043488 \\
\hline $\mathrm{C}$ & -3.253542 & -1.299771 & -0.629756 \\
\hline $\mathrm{C}$ & 3.029413 & 1.298965 & -0.943868 \\
\hline $\mathrm{C}$ & 1.211905 & -3.141946 & -0.090458 \\
\hline $\mathrm{C}$ & -1.375433 & 3.136629 & 0.034594 \\
\hline Mn & -0.086276 & 0.000604 & -0.371951 \\
\hline $\mathrm{Cl}$ & -0.231910 & -0.011501 & -2.716789 \\
\hline O & -0.134106 & -0.058578 & 1.347244 \\
\hline $\mathrm{C}$ & 0.535991 & -0.496932 & 4.913003 \\
\hline C & 0.141769 & 0.809519 & 4.532299 \\
\hline $\mathrm{C}$ & 0.545733 & 1.352132 & 3.333413 \\
\hline $\mathrm{C}$ & 1.317166 & 0.564196 & 2.415485 \\
\hline $\mathrm{C}$ & 1.822925 & -0.698508 & 2.865065 \\
\hline $\mathrm{C}$ & 1.396939 & -1.227303 & 4.065690 \\
\hline $\mathrm{H}$ & -0.487148 & 1.386598 & 5.205888 \\
\hline $\mathrm{H}$ & 0.228483 & 2.344113 & 3.031107 \\
\hline $\mathrm{H}$ & 1.844971 & 1.077016 & 1.622314 \\
\hline $\mathrm{H}$ & 2.488563 & -1.259935 & 2.221437 \\
\hline $\mathrm{H}$ & 1.730218 & -2.216743 & 4.366665 \\
\hline $\mathrm{C}$ & 0.028217 & -1.097667 & 6.195535 \\
\hline $\mathrm{H}$ & -0.076439 & -0.341177 & 6.981361 \\
\hline $\mathrm{H}$ & -0.965162 & -1.542673 & 6.042639 \\
\hline $\mathrm{H}$ & 0.688198 & -1.890863 & 6.561484 \\
\hline $\mathrm{C}$ & 1.802150 & -4.477446 & 0.208600 \\
\hline $\mathrm{C}$ & 2.417864 & -4.726251 & 1.439475 \\
\hline $\mathrm{C}$ & 1.790584 & -5.521899 & -0.721056 \\
\hline $\mathrm{C}$ & 2.993513 & -5.956839 & 1.743330 \\
\hline $\mathrm{C}$ & 2.352415 & -6.765429 & -0.438048 \\
\hline $\mathrm{C}$ & 2.957825 & -6.981272 & 0.798738 \\
\hline $\mathrm{C}$ & -4.630184 & -1.842509 & -0.809480 \\
\hline $\mathrm{C}$ & -5.338112 & -1.617118 & -1.994871 \\
\hline $\mathrm{C}$ & -5.276515 & -2.566069 & 0.197196 \\
\hline C & -6.633177 & -2.098271 & -2.178021 \\
\hline C & -6.567314 & -3.062591 & 0.033936 \\
\hline $\mathrm{C}$ & -7.247391 & -2.824476 & -1.159245 \\
\hline $\mathrm{C}$ & -1.888962 & 4.493286 & 0.373099 \\
\hline & -1.523897 & 5.115360 & 1.57152 \\
\hline
\end{tabular}




\begin{tabular}{|c|c|c|c|}
\hline C & -2.726862 & 5.210934 & -0.487171 \\
\hline C & -1.974252 & 6.387611 & 1.913102 \\
\hline $\mathrm{C}$ & -3.197064 & 6.482526 & -0.164970 \\
\hline $\mathrm{C}$ & -2.817091 & 7.072438 & 1.039359 \\
\hline C & 4.384583 & 1.853447 & -1.216460 \\
\hline $\mathrm{C}$ & 5.013406 & 1.664918 & -2.450799 \\
\hline $\mathrm{C}$ & 5.085365 & 2.563763 & -0.237263 \\
\hline $\mathrm{C}$ & 6.287223 & 2.169438 & -2.707080 \\
\hline $\mathrm{C}$ & 6.355781 & 3.082636 & -0.472078 \\
\hline $\mathrm{C}$ & 6.957345 & 2.881763 & -1.713834 \\
\hline $\mathrm{F}$ & -1.608515 & 6.951516 & 3.068932 \\
\hline $\mathrm{F}$ & -0.708349 & 4.487031 & 2.434808 \\
\hline F & -3.259003 & 8.290976 & 1.355582 \\
\hline $\mathrm{F}$ & -3.999824 & 7.142569 & -1.005141 \\
\hline $\mathrm{F}$ & -3.100406 & 4.676891 & -1.655736 \\
\hline F & 4.530040 & 2.760109 & 0.968343 \\
\hline F & 4.393557 & 0.980628 & -3.416830 \\
\hline F & 6.868234 & 1.977824 & -3.895220 \\
\hline F & 8.176315 & 3.370676 & -1.950638 \\
\hline F & 7.003018 & 3.760594 & 0.481783 \\
\hline F & 2.473593 & -3.759298 & 2.371530 \\
\hline F & 3.573485 & -6.162071 & 2.930702 \\
\hline F & 3.502131 & -8.166841 & 1.078921 \\
\hline F & 2.322281 & -7.746642 & -1.344643 \\
\hline F & 1.221866 & -5.342143 & -1.918872 \\
\hline F & -4.649822 & -2.801582 & 1.358566 \\
\hline F & -4.774883 & -0.919217 & -2.985464 \\
\hline F & -7.288172 & -1.870419 & -3.320610 \\
\hline F & -8.486951 & -3.291029 & -1.325372 \\
\hline$F$ & -7.159269 & -3.754830 & 1.01370 \\
\hline
\end{tabular}

\section{Structure: ${ }^{5}$ TS4-m1}

$\mathrm{C}$
$\mathrm{C}$
$\mathrm{C}$
$\mathrm{C}$
$\mathrm{N}$
$\mathrm{H}$
$\mathrm{H}$
$\mathrm{C}$
$\mathrm{C}$
$\mathrm{C}$

$-2.289646$

$-2.128099$

$-0.543490$

$-2.472193$

$-3.559856$

$-0.469703$

$-1.255630$

$-4.104313$

$-0.209145$

$-0.300426$

$-3.020652$

$-0.191595$

$-0.960479$

$-1.831219$

$-0.391791$

$-3.415568$

$-4.073989$

$-0.578652$

$-1.017950$

$-5.148493$

$-0.070846$

2.884526

$-0.190206$

$-0.802176$

3.971506

$-1.139267$

$-0.760205$

3.433120

$-2.346851$

$-0.436585$ 
C

N

$\mathrm{H}$

$\mathrm{H}$

C

C

C

C

$\mathrm{N}$

$\mathrm{H}$

$\mathrm{H}$

C

C

C

C

$\mathrm{N}$

$\mathrm{H}$

$\mathrm{H}$

C

C

C

C

$\mathrm{Mn}$

$\mathrm{Cl}$

O

C

C

C

C

C

C

$\mathrm{H}$

$\mathrm{H}$

$\mathrm{H}$

$\mathrm{H}$

$\mathrm{H}$

C

$\mathrm{H}$

$\mathrm{H}$

$\mathrm{H}$

C

C

C

C

\begin{tabular}{|c|c|c|}
\hline 2.007793 & -2.151389 & -0.309353 \\
\hline 1.707136 & -0.835457 & -0.527075 \\
\hline 5.010348 & -0.902711 & -0.939432 \\
\hline 3.944119 & -3.292097 & -0.324085 \\
\hline-2.222290 & 2.141071 & -0.032908 \\
\hline 3.652557 & 2.317198 & 0.067777 \\
\hline-4.2230 & 1.119183 & -0.223102 \\
\hline-3.1473 & 0.179690 & -0.445973 \\
\hline-1.94722 & 0.825918 & -0.330365 \\
\hline-4.1518 & 3.243827 & 0.309027 \\
\hline-5.275 & 0.87 & -0.250323 \\
\hline 0.079761 & 3.010 & -0.23 \\
\hline 1.005333 & 4.10 & -0.43 \\
\hline 2.1910 & 3.55 & -0.8 \\
\hline 2.014 & 2.12 & -0.8 \\
\hline 0.716667 & 1.82 & -0.4 \\
\hline 0.7616 & 5.14 & -0.327944 \\
\hline 3.1071 & 4.06 & -1.063483 \\
\hline-3.3275 & -1.19 & -0.621184 \\
\hline 3.039392 & 1.187781 & -0.987065 \\
\hline 1.0820 & -3.17 & -0.086900 \\
\hline-1.2827 & & \\
\hline-0.13114 & -0.009062 & -0.409098 \\
\hline-0.1845 & 0.02 & -2.7 \\
\hline-0.0875 & -0.02 & 1.3 \\
\hline 0.735776 & -0.49 & \\
\hline 0.376306 & 0.825648 & 4.565200 \\
\hline 0.803083 & 1.3804 & 3.373702 \\
\hline 1.59012 & $0.60 s$ & \\
\hline 2.011872 & -0.68717 & 2.865295 \\
\hline 1.567259 & -1.231798 & 4.055894 \\
\hline-0.249985 & 1.40 & 0018 \\
\hline 0.504263 & 2.38274 & 3.086850 \\
\hline 2.045192 & 1.088042 & 1.622205 \\
\hline 2.647817 & -1.261765 & 2.202277 \\
\hline 1.856344 & -2.243366 & 4.326767 \\
\hline 0.211742 & -1.107353 & 6.192153 \\
\hline 0.089236 & -0.358713 & 6.982502 \\
\hline-0.774637 & -1.560273 & 6.019663 \\
\hline 0.872782 & -1.898106 & 6.561925 \\
\hline 1.628459 & -4.530891 & 0.221749 \\
\hline 2.258231 & -4.781268 & 1.445263 \\
\hline 1.565763 & -5.588213 & -0.691114 \\
\hline 2.796622 & -6.0260 & 1.759037 \\
\hline
\end{tabular}




\begin{tabular}{|c|c|c|c|}
\hline C & 2.089674 & -6.845828 & -0.397718 \\
\hline C & 2.709032 & -7.063251 & 0.831863 \\
\hline $\mathrm{C}$ & -4.722621 & -1.688324 & -0.798086 \\
\hline C & -5.426569 & -1.428112 & -1.978884 \\
\hline C & -5.392003 & -2.394567 & 0.205982 \\
\hline C & -6.739211 & -1.859367 & -2.160555 \\
\hline C & -6.700833 & -2.841937 & 0.043989 \\
\hline $\mathrm{C}$ & -7.376103 & -2.570046 & -1.144712 \\
\hline $\mathrm{C}$ & -1.747524 & 4.539009 & 0.406718 \\
\hline $\mathrm{C}$ & -1.354873 & 5.135916 & 1.609133 \\
\hline $\mathrm{C}$ & -2.561549 & 5.294337 & -0.443901 \\
\hline $\mathrm{C}$ & -1.757367 & 6.420708 & 1.963576 \\
\hline $\mathrm{C}$ & -2.983559 & 6.579405 & -0.108849 \\
\hline C & -2.577681 & 7.143804 & 1.099251 \\
\hline C & 4.410414 & 1.694314 & -1.274720 \\
\hline $\mathrm{C}$ & 5.015432 & 1.487728 & -2.517924 \\
\hline $\mathrm{C}$ & 5.149433 & 2.373861 & -0.302011 \\
\hline $\mathrm{C}$ & 6.303491 & 1.946136 & -2.789337 \\
\hline $\mathrm{C}$ & 6.435113 & 2.846095 & -0.551656 \\
\hline $\mathrm{C}$ & 7.012395 & 2.628784 & -1.802203 \\
\hline $\mathrm{F}$ & -1.367492 & 6.960544 & 3.122941 \\
\hline $\mathrm{F}$ & -0.560377 & 4.469993 & 2.463041 \\
\hline $\mathrm{F}$ & -2.973630 & 8.374653 & 1.428280 \\
\hline $\mathrm{F}$ & -3.764853 & 7.275872 & -0.939843 \\
\hline F & -2.958811 & 4.783956 & -1.615372 \\
\hline F & 4.617472 & 2.585160 & 0.911805 \\
\hline $\mathrm{F}$ & 4.357759 & 0.831024 & -3.477914 \\
\hline F & 6.861192 & 1.738352 & -3.985854 \\
\hline F & 8.245368 & 3.073524 & -2.053002 \\
\hline $\mathrm{F}$ & 7.119872 & 3.495108 & 0.396091 \\
\hline $\mathrm{F}$ & 2.364577 & -3.801478 & 2.358606 \\
\hline F & 3.390906 & -6.232494 & 2.939065 \\
\hline F & 3.217381 & -8.262385 & 1.121588 \\
\hline F & 2.010336 & -7.839307 & -1.287902 \\
\hline F & 0.983666 & -5.407478 & -1.882563 \\
\hline $\mathrm{F}$ & -4.770740 & -2.661135 & 1.363576 \\
\hline F & -4.841453 & -0.744004 & -2.966709 \\
\hline F & -7.389347 & -1.598828 & -3.298835 \\
\hline F & -8.632534 & -2.989274 & -1.309318 \\
\hline F & -7.314415 & -3.518809 & 1.021038 \\
\hline
\end{tabular}

\section{Structure: ${ }^{3} \mathrm{TS} 2 \mathrm{~b}-\mathrm{m} 2$}




\begin{tabular}{|c|c|c|c|}
\hline C & -0.641984 & -1.767173 & -2.973894 \\
\hline C & -1.660966 & -1.735334 & -3.990435 \\
\hline $\mathrm{C}$ & -2.864479 & -1.802368 & -3.354017 \\
\hline C & -2.593205 & -1.871610 & -1.942080 \\
\hline $\mathrm{N}$ & -1.233378 & -1.825162 & -1.731181 \\
\hline $\mathrm{H}$ & -1.462348 & -1.684677 & -5.053238 \\
\hline $\mathrm{H}$ & -3.857048 & -1.811076 & -3.785324 \\
\hline C & -2.285120 & -2.072259 & 2.343962 \\
\hline $\mathrm{C}$ & -3.700942 & -2.118527 & 2.621954 \\
\hline $\mathrm{C}$ & -4.343868 & -2.097158 & 1.421508 \\
\hline C & -3.323843 & -2.035772 & 0.402447 \\
\hline $\mathrm{N}$ & -2.086135 & -2.025778 & 0.988292 \\
\hline $\mathrm{H}$ & -4.128130 & -2.161463 & 3.615552 \\
\hline $\mathrm{H}$ & -5.407547 & -2.112821 & 1.222696 \\
\hline $\mathrm{C}$ & 2.737295 & -1.882355 & -0.312045 \\
\hline C & 3.764775 & -1.878036 & -1.325038 \\
\hline C & 3.132612 & -1.858808 & -2.531964 \\
\hline C & 1.714304 & -1.849473 & -2.262193 \\
\hline $\mathrm{N}$ & 1.502342 & -1.879276 & -0.909791 \\
\hline $\mathrm{H}$ & 4.823750 & -1.857497 & -1.108299 \\
\hline $\mathrm{H}$ & 3.566844 & -1.844570 & -3.523511 \\
\hline $\mathrm{C}$ & 2.018512 & -1.919477 & 2.042662 \\
\hline $\mathrm{C}$ & 2.290891 & -1.917201 & 3.459747 \\
\hline $\mathrm{C}$ & 1.088744 & -1.981228 & 4.096531 \\
\hline C & 0.071405 & -2.017251 & 3.073257 \\
\hline $\mathrm{N}$ & 0.665110 & -1.986927 & 1.839150 \\
\hline $\mathrm{H}$ & 3.283097 & -1.876407 & 3.890044 \\
\hline $\mathrm{C}$ & 0.721316 & -1.777113 & -3.229842 \\
\hline C & -1.294105 & -2.066895 & 3.315647 \\
\hline C & -3.569772 & -1.965518 & -0.961031 \\
\hline $\mathrm{C}$ & 2.988147 & -1.875810 & 1.051779 \\
\hline $\mathrm{Mn}$ & -0.291088 & -1.898730 & 0.044983 \\
\hline $\mathrm{Cl}$ & -0.238074 & -4.234612 & -0.035393 \\
\hline $\mathrm{C}$ & 3.920674 & 2.460655 & -0.272977 \\
\hline C & 3.244369 & 3.465859 & 0.372571 \\
\hline C & 1.835329 & 3.571041 & 0.239756 \\
\hline C & 1.122884 & 2.617101 & -0.557864 \\
\hline C & 1.876049 & 1.609301 & -1.216515 \\
\hline $\mathrm{C}$ & 3.245287 & 1.533894 & -1.092467 \\
\hline $\mathrm{H}$ & 3.790152 & 4.175319 & 0.987202 \\
\hline C & 1.111659 & 4.592336 & 0.908171 \\
\hline $\mathrm{C}$ & -0.311494 & 2.694326 & -0.643080 \\
\hline $\mathrm{H}$ & 1.376733 & 0.861777 & -1.818048 \\
\hline $\mathrm{H}$ & 3.798130 & 0.754759 & -1.598671 \\
\hline
\end{tabular}




\begin{tabular}{|c|c|c|c|}
\hline C & -0.982999 & 3.716012 & 0.045519 \\
\hline C & -0.259414 & 4.662001 & 0.803964 \\
\hline 0 & 5.314411 & 2.441440 & -0.151169 \\
\hline C & 7.446268 & 1.527190 & 0.228923 \\
\hline $\mathrm{H}$ & 7.955128 & 0.611329 & 0.530973 \\
\hline $\mathrm{H}$ & 7.794243 & 1.840696 & -0.760985 \\
\hline $\mathrm{H}$ & 7.679938 & 2.336548 & 0.928035 \\
\hline C & 5.956909 & 1.286706 & 0.195567 \\
\hline O & 5.404221 & 0.239109 & 0.436250 \\
\hline$C$ & -2.480523 & 3.800463 & -0.095898 \\
\hline C & -3.126441 & 2.401066 & -0.158884 \\
\hline C & -3.344702 & 4.582877 & 0.911968 \\
\hline $\mathrm{H}$ & -3.227482 & 5.666878 & 0.816639 \\
\hline $\mathrm{C}$ & -4.776704 & 4.110315 & 0.560076 \\
\hline $\mathrm{H}$ & -5.274415 & 4.797724 & -0.135107 \\
\hline $\mathrm{C}$ & -4.610371 & 2.756693 & -0.162254 \\
\hline O & -5.507889 & 2.096640 & -0.640087 \\
\hline $\mathrm{C}$ & -2.903063 & 1.554873 & 1.121821 \\
\hline $\mathrm{H}$ & -3.448228 & 0.611965 & 1.026644 \\
\hline $\mathrm{H}$ & -1.847683 & 1.316202 & 1.269027 \\
\hline $\mathrm{H}$ & -3.269574 & 2.064860 & 2.019347 \\
\hline $\mathrm{H}$ & -5.441563 & 4.001399 & 1.423201 \\
\hline $\mathrm{H}$ & -3.078990 & 4.315587 & 1.940480 \\
\hline C & -2.590752 & 1.690925 & -1.397987 \\
\hline C & -1.060744 & 1.611428 & -1.343142 \\
\hline $\mathrm{H}$ & -2.897887 & 2.247614 & -2.295684 \\
\hline $\mathrm{H}$ & -3.013985 & 0.688028 & -1.496566 \\
\hline $\mathrm{H}$ & -0.635352 & 1.338603 & -2.313986 \\
\hline $\mathrm{H}$ & -0.776118 & 0.658155 & -0.684194 \\
\hline $\mathrm{H}$ & -0.801097 & 5.449638 & 1.319588 \\
\hline $\mathrm{H}$ & 1.655497 & 5.314555 & 1.512131 \\
\hline $\mathrm{H}$ & 0.888043 & -2.003211 & 5.159949 \\
\hline O & -0.234745 & -0.201797 & 0.310085 \\
\hline $\mathrm{H}$ & -2.674740 & 4.263294 & -1.080698 \\
\hline $\mathrm{H}$ & -1.614434 & -2.098361 & 4.351984 \\
\hline $\mathrm{H}$ & 4.024790 & -1.809559 & 1.360672 \\
\hline $\mathrm{H}$ & 1.034212 & -1.741297 & -4.268218 \\
\hline $\mathrm{H}$ & -4.604701 & -1.974655 & -1.285799 \\
\hline
\end{tabular}

\section{Structure: ${ }^{5} \mathrm{TS} 2 \mathrm{~b}-\mathrm{m} 2$}

$$
\begin{array}{clll}
\text { C } & -0.564140 & -1.734175 & -2.971220 \\
\text { C } & -1.569347 & -1.698581 & -4.003712 \\
\text { S208 } & &
\end{array}
$$




\begin{tabular}{|c|c|c|c|}
\hline $\mathrm{C}$ & -2.779809 & -1.781277 & -3.380283 \\
\hline C & -2.515690 & -1.865039 & -1.966259 \\
\hline $\mathrm{N}$ & -1.165903 & -1.809878 & -1.742337 \\
\hline $\mathrm{H}$ & -1.359468 & -1.636484 & -5.063753 \\
\hline $\mathrm{H}$ & -3.767936 & -1.794765 & -3.821529 \\
\hline C & -2.258549 & -2.168371 & 2.327630 \\
\hline $\mathrm{C}$ & -3.678216 & -2.232471 & 2.586111 \\
\hline C & -4.302111 & -2.188651 & 1.376840 \\
\hline C & -3.266248 & -2.098180 & 0.374183 \\
\hline $\mathrm{N}$ & -2.035214 & -2.097626 & 0.982400 \\
\hline $\mathrm{H}$ & -4.119879 & -2.296881 & 3.572141 \\
\hline $\mathrm{H}$ & -5.362560 & -2.204924 & 1.161418 \\
\hline C & 2.802330 & -1.858723 & -0.277705 \\
\hline C & 3.837964 & -1.831934 & -1.282875 \\
\hline C & 3.211586 & -1.810538 & -2.492509 \\
\hline C & 1.790704 & -1.821630 & -2.229953 \\
\hline $\mathrm{N}$ & 1.573571 & -1.871263 & -0.876004 \\
\hline $\mathrm{H}$ & 4.895226 & -1.796960 & -1.060186 \\
\hline $\mathrm{H}$ & 3.650772 & -1.779546 & -3.481540 \\
\hline $\mathrm{C}$ & 2.049219 & -1.902417 & 2.064420 \\
\hline C & 2.300792 & -1.899877 & 3.483191 \\
\hline C & 1.093985 & -1.998639 & 4.106284 \\
\hline $\mathrm{C}$ & 0.087664 & -2.055129 & 3.076360 \\
\hline $\mathrm{N}$ & 0.692667 & -1.996507 & 1.839264 \\
\hline $\mathrm{H}$ & 3.286268 & -1.837375 & 3.926182 \\
\hline C & 0.807251 & -1.738325 & -3.204786 \\
\hline C & -1.273724 & -2.151434 & 3.309741 \\
\hline C & -3.497897 & -1.991524 & -0.987506 \\
\hline $\mathrm{C}$ & 3.033218 & -1.847360 & 1.092409 \\
\hline Mn & -0.224051 & -1.932750 & 0.076429 \\
\hline $\mathrm{Cl}$ & -0.211751 & -4.257400 & -0.196844 \\
\hline $\mathrm{C}$ & 3.855999 & 2.529908 & -0.271160 \\
\hline $\mathrm{C}$ & 3.166340 & 3.532467 & 0.366540 \\
\hline C & 1.756792 & 3.618450 & 0.232743 \\
\hline C & 1.057652 & 2.652717 & -0.561639 \\
\hline C & 1.822348 & 1.647641 & -1.209231 \\
\hline C & 3.193197 & 1.588027 & -1.081856 \\
\hline $\mathrm{H}$ & 3.703128 & 4.252316 & 0.976993 \\
\hline C & 1.019550 & 4.630028 & 0.902101 \\
\hline C & -0.378826 & 2.709162 & -0.648691 \\
\hline $\mathrm{H}$ & 1.331786 & 0.887743 & -1.802080 \\
\hline $\mathrm{H}$ & 3.755604 & 0.808791 & -1.577654 \\
\hline C & -1.064418 & 3.720139 & 0.049286 \\
\hline C & -0.354217 & 4.675950 & 0.804790 \\
\hline
\end{tabular}




\begin{tabular}{|c|c|c|c|}
\hline 0 & 5.249469 & 2.529211 & -0.148293 \\
\hline C & 7.391429 & 1.644348 & 0.245112 \\
\hline $\mathrm{H}$ & 7.910120 & 0.741449 & 0.568712 \\
\hline $\mathrm{H}$ & 7.734676 & 1.936522 & -0.752977 \\
\hline $\mathrm{H}$ & 7.617900 & 2.473236 & 0.923343 \\
\hline C & 5.904647 & 1.387880 & 0.219757 \\
\hline O & 5.362886 & 0.339881 & 0.481862 \\
\hline C & -2.563195 & 3.775780 & -0.085182 \\
\hline C & -3.181925 & 2.363761 & -0.145965 \\
\hline C & -3.436797 & 4.541528 & 0.927157 \\
\hline $\mathrm{H}$ & -3.341054 & 5.627673 & 0.831703 \\
\hline C & -4.861158 & 4.041199 & 0.583635 \\
\hline $\mathrm{H}$ & -5.374658 & 4.717387 & -0.111165 \\
\hline $\mathrm{C}$ & -4.672671 & 2.689151 & -0.135962 \\
\hline O & -5.561050 & 2.008607 & -0.602225 \\
\hline $\mathrm{C}$ & -2.930448 & 1.517246 & 1.129860 \\
\hline $\mathrm{H}$ & -3.470707 & 0.570476 & 1.041354 \\
\hline $\mathrm{H}$ & -1.871175 & 1.283803 & 1.257947 \\
\hline $\mathrm{H}$ & -3.285403 & 2.022850 & 2.034598 \\
\hline $\mathrm{H}$ & -5.519935 & 3.922411 & 1.450098 \\
\hline $\mathrm{H}$ & -3.160193 & 4.278914 & 1.954086 \\
\hline C & -2.641285 & 1.667311 & -1.391046 \\
\hline C & -1.110705 & 1.621478 & -1.346358 \\
\hline $\mathrm{H}$ & -2.974073 & 2.213660 & -2.286340 \\
\hline $\mathrm{H}$ & -3.041820 & 0.654270 & -1.482999 \\
\hline $\mathrm{H}$ & -0.681378 & 1.350412 & -2.316040 \\
\hline $\mathrm{H}$ & -0.794800 & 0.664499 & -0.678546 \\
\hline $\mathrm{H}$ & -0.906315 & 5.454348 & 1.323423 \\
\hline $\mathrm{H}$ & 1.553883 & 5.361478 & 1.503342 \\
\hline $\mathrm{H}$ & 0.883894 & -2.032718 & 5.167545 \\
\hline O & -0.242755 & -0.200183 & 0.282820 \\
\hline $\mathrm{H}$ & -2.770485 & 4.234010 & -1.069631 \\
\hline $\mathrm{H}$ & -1.599110 & -2.202411 & 4.343729 \\
\hline $\mathrm{H}$ & 4.063223 & -1.764469 & 1.418919 \\
\hline $\mathrm{H}$ & 1.134479 & -1.686898 & -4.238410 \\
\hline $\mathrm{H}$ & -4.530978 & -2.000835 & -1.319150 \\
\hline
\end{tabular}

\section{Structure: ${ }^{3} \mathrm{TS} 4-\mathrm{m} 2$}

C
C
C
C

$$
\begin{array}{rrr}
1.332453 & -2.826596 & -1.973889 \\
1.180959 & -3.340153 & -3.312502 \\
-0.151337 & -3.322185 & -3.599385 \\
-0.824708 & -2.776098 & -2.448058
\end{array}
$$




\begin{tabular}{|c|c|c|c|}
\hline $\mathrm{N}$ & 0.100841 & -2.471797 & -1.476073 \\
\hline $\mathrm{H}$ & 1.998482 & -3.688394 & -3.930707 \\
\hline $\mathrm{H}$ & -0.649932 & -3.642803 & -4.505117 \\
\hline $\mathrm{C}$ & -3.183800 & -0.878130 & 0.608917 \\
\hline C & -4.444753 & -0.912791 & -0.090716 \\
\hline C & -4.232801 & -1.572201 & -1.266080 \\
\hline $\mathrm{C}$ & -2.833882 & -1.925895 & -1.301247 \\
\hline $\mathrm{N}$ & -2.225194 & -1.499717 & -0.150170 \\
\hline $\mathrm{H}$ & -5.365386 & -0.499149 & 0.298757 \\
\hline $\mathrm{H}$ & -4.943337 & -1.802143 & -2.049844 \\
\hline $\mathrm{C}$ & 2.219914 & -1.776656 & 2.106738 \\
\hline C & 3.636549 & -2.058500 & 2.059740 \\
\hline $\mathrm{C}$ & 3.924923 & -2.432657 & 0.781578 \\
\hline C & 2.680927 & -2.402931 & 0.046407 \\
\hline $\mathrm{N}$ & 1.668443 & -1.998911 & 0.872358 \\
\hline $\mathrm{H}$ & 4.308214 & -1.966868 & 2.903641 \\
\hline $\mathrm{H}$ & 4.879829 & -2.722498 & 0.361892 \\
\hline C & 0.217114 & -0.904679 & 3.253760 \\
\hline C & -0.427280 & -0.258076 & 4.374940 \\
\hline $\mathrm{C}$ & -1.691084 & 0.055488 & 3.978209 \\
\hline C & -1.836011 & -0.420693 & 2.621421 \\
\hline $\mathrm{N}$ & -0.665291 & -1.002262 & 2.214448 \\
\hline $\mathrm{H}$ & 0.048759 & -0.065928 & 5.327899 \\
\hline $\mathrm{H}$ & -2.473214 & 0.551204 & 4.538399 \\
\hline C & 2.537570 & -2.766715 & -1.286944 \\
\hline $\mathrm{C}$ & -3.003430 & -0.339004 & 1.876342 \\
\hline $\mathrm{C}$ & -2.190563 & -2.539000 & -2.370402 \\
\hline $\mathrm{C}$ & 1.551079 & -1.291875 & 3.222641 \\
\hline $\mathrm{Mn}$ & -0.278878 & -1.710737 & 0.350186 \\
\hline $\mathrm{Cl}$ & -0.753074 & -3.867255 & 1.154502 \\
\hline 0 & 0.056737 & -0.078276 & -0.033213 \\
\hline C & -3.143745 & 2.779470 & -0.900938 \\
\hline $\mathrm{C}$ & -2.402537 & 1.743505 & -1.430351 \\
\hline C & -1.000487 & 1.809875 & -1.402663 \\
\hline C & -0.337439 & 2.935946 & -0.833513 \\
\hline $\mathrm{C}$ & -1.142981 & 3.973694 & -0.299616 \\
\hline $\mathrm{C}$ & -2.524628 & 3.900759 & -0.329794 \\
\hline $\mathrm{H}$ & -2.905959 & 0.873641 & -1.837903 \\
\hline $\mathrm{C}$ & -0.206432 & 0.695716 & -1.846369 \\
\hline $\mathrm{C}$ & 1.105742 & 2.960075 & -0.790445 \\
\hline $\mathrm{H}$ & -0.680491 & 4.847527 & 0.144689 \\
\hline $\mathrm{H}$ & -3.137302 & 4.696112 & 0.081021 \\
\hline $\mathrm{C}$ & 1.840656 & 1.941515 & -1.419700 \\
\hline $\mathrm{C}$ & 1.184384 & 0.851548 & -1.998479 \\
\hline
\end{tabular}




\begin{tabular}{|c|c|c|c|}
\hline 0 & -4.536456 & 2.716635 & -0.999316 \\
\hline C & -6.718770 & 2.302334 & -0.222279 \\
\hline $\mathrm{H}$ & -7.302350 & 2.179445 & 0.690662 \\
\hline $\mathrm{H}$ & -7.052205 & 3.186990 & -0.772882 \\
\hline $\mathrm{H}$ & -6.865028 & 1.435093 & -0.875902 \\
\hline C & -5.256059 & 2.417222 & 0.126008 \\
\hline O & -4.767866 & 2.253772 & 1.217598 \\
\hline C & 3.345330 & 1.994660 & -1.345903 \\
\hline C & 3.795235 & 2.514146 & 0.038600 \\
\hline C & 4.184988 & 0.721327 & -1.568458 \\
\hline $\mathrm{H}$ & 4.237278 & 0.430730 & -2.622693 \\
\hline $\mathrm{C}$ & 5.566125 & 1.112900 & -0.991779 \\
\hline $\mathrm{H}$ & 6.259372 & 1.461858 & -1.766731 \\
\hline $\mathrm{C}$ & 5.304526 & 2.292057 & -0.033090 \\
\hline O & 6.152938 & 2.927617 & 0.554112 \\
\hline C & 3.289615 & 1.630979 & 1.210019 \\
\hline $\mathrm{H}$ & 3.606973 & 2.076150 & 2.159114 \\
\hline $\mathrm{H}$ & 2.200283 & 1.533672 & 1.205007 \\
\hline $\mathrm{H}$ & 3.695502 & 0.615754 & 1.166620 \\
\hline $\mathrm{H}$ & 6.070975 & 0.299366 & -0.459678 \\
\hline $\mathrm{H}$ & 3.755918 & -0.121679 & -1.019645 \\
\hline C & 3.326491 & 3.958974 & 0.170019 \\
\hline C & 1.784900 & 4.044833 & 0.034220 \\
\hline $\mathrm{H}$ & 3.807107 & 4.556207 & -0.614747 \\
\hline $\mathrm{H}$ & 3.640815 & 4.390643 & 1.126008 \\
\hline $\mathrm{H}$ & 1.513954 & 5.033857 & -0.359339 \\
\hline $\mathrm{H}$ & 1.335019 & 4.000717 & 1.035519 \\
\hline $\mathrm{H}$ & 1.754183 & 0.032670 & -2.422727 \\
\hline $\mathrm{H}$ & -0.708721 & -0.133665 & -2.327538 \\
\hline $\mathrm{H}$ & 3.696595 & 2.739022 & -2.081187 \\
\hline $\mathrm{H}$ & -3.850459 & 0.169598 & 2.321143 \\
\hline $\mathrm{H}$ & -2.796398 & -2.821819 & -3.225061 \\
\hline $\mathrm{H}$ & 2.130738 & -1.155659 & 4.129868 \\
\hline & 3.430066 & -3.080189 & -1.818830 \\
\hline
\end{tabular}

\section{Structure: ${ }^{5}$ TS4-m2}

$\begin{array}{crrr}\mathrm{C} & 1.434609 & -2.786080 & -1.951601 \\ \mathrm{C} & 1.303426 & -3.294684 & -3.295466 \\ \mathrm{C} & -0.028927 & -3.308349 & -3.585499 \\ \mathrm{C} & -0.715814 & -2.787320 & -2.428991 \\ \mathrm{~N} & 0.198077 & -2.467042 & -1.456936 \\ \mathrm{H} & 2.131007 & -3.619287 & -3.913188\end{array}$




\begin{tabular}{|c|c|c|c|}
\hline $\mathrm{H}$ & -0.517657 & -3.636455 & -4.493960 \\
\hline $\mathrm{C}$ & -3.129244 & -0.928928 & 0.611630 \\
\hline $\mathrm{C}$ & -4.390501 & -1.005263 & -0.086830 \\
\hline C & -4.159992 & -1.678300 & -1.250229 \\
\hline C & -2.751023 & -1.998197 & -1.276865 \\
\hline N & -2.158116 & -1.545511 & -0.127033 \\
\hline $\mathrm{H}$ & -5.321669 & -0.607712 & 0.294280 \\
\hline $\mathrm{H}$ & -4.862469 & -1.935439 & -2.032853 \\
\hline C & 2.291034 & -1.740040 & 2.147699 \\
\hline $\mathrm{C}$ & 3.713391 & -1.991674 & 2.098696 \\
\hline $\mathrm{C}$ & 4.007130 & -2.355552 & 0.819206 \\
\hline $\mathrm{C}$ & 2.762114 & -2.353190 & 0.084828 \\
\hline $\mathrm{N}$ & 1.739298 & -1.977710 & 0.918358 \\
\hline $\mathrm{H}$ & 4.385123 & -1.882598 & 2.940405 \\
\hline $\mathrm{H}$ & 4.968124 & -2.620161 & 0.396697 \\
\hline $\mathrm{C}$ & 0.279769 & -0.855195 & 3.258792 \\
\hline $\mathrm{C}$ & -0.377288 & -0.188242 & 4.356801 \\
\hline C & -1.640161 & 0.112537 & 3.944096 \\
\hline C & -1.778759 & -0.393534 & 2.601018 \\
\hline $\mathrm{N}$ & -0.594158 & -0.977004 & 2.208257 \\
\hline $\mathrm{H}$ & 0.089325 & 0.025543 & 5.309827 \\
\hline $\mathrm{H}$ & -2.427377 & 0.616439 & 4.489644 \\
\hline $\mathrm{C}$ & 2.633896 & -2.706085 & -1.251048 \\
\hline $\mathrm{C}$ & -2.950315 & -0.349596 & 1.864068 \\
\hline C & -2.090121 & -2.592021 & -2.345118 \\
\hline $\mathrm{C}$ & 1.612317 & -1.244080 & 3.253139 \\
\hline Mn & -0.204544 & -1.730505 & 0.403549 \\
\hline $\mathrm{Cl}$ & -0.663539 & -3.922851 & 1.099258 \\
\hline 0 & 0.122184 & -0.091173 & -0.092053 \\
\hline $\mathrm{C}$ & -3.245962 & 2.651738 & -0.896025 \\
\hline C & -2.472374 & 1.671535 & -1.480581 \\
\hline C & -1.071976 & 1.795335 & -1.475230 \\
\hline $\mathrm{C}$ & -0.445774 & 2.924499 & -0.867097 \\
\hline C & -1.286010 & 3.904572 & -0.279053 \\
\hline C & -2.663302 & 3.775038 & -0.289318 \\
\hline $\mathrm{H}$ & -2.947373 & 0.798288 & -1.914008 \\
\hline $\mathrm{C}$ & -0.246499 & 0.747545 & -1.992481 \\
\hline $\mathrm{C}$ & 0.993143 & 3.001044 & -0.828875 \\
\hline $\mathrm{H}$ & -0.852816 & 4.778701 & 0.193508 \\
\hline $\mathrm{H}$ & -3.299828 & 4.526097 & 0.165582 \\
\hline C & 1.759409 & 2.024369 & -1.484765 \\
\hline C & 1.136325 & 0.936798 & -2.106613 \\
\hline O & -4.634977 & 2.529594 & -0.980103 \\
\hline $\mathrm{C}$ & -6.804246 & 2.111381 & -0.171958 \\
\hline
\end{tabular}




\begin{tabular}{|c|c|c|c|}
\hline $\mathrm{H}$ & -7.378630 & 1.996252 & 0.747738 \\
\hline $\mathrm{H}$ & -7.164226 & 2.974164 & -0.740626 \\
\hline $\mathrm{H}$ & -6.932204 & 1.226249 & -0.804852 \\
\hline $\mathrm{C}$ & -5.343193 & 2.276016 & 0.163026 \\
\hline O & -4.848005 & 2.183521 & 1.260062 \\
\hline C & 3.261275 & 2.098685 & -1.380579 \\
\hline C & 3.675546 & 2.589133 & 0.025542 \\
\hline C & 4.121431 & 0.840453 & -1.614120 \\
\hline $\mathrm{H}$ & 4.202956 & 0.577147 & -2.673652 \\
\hline C & 5.484168 & 1.231818 & -0.994333 \\
\hline $\mathrm{H}$ & 6.197092 & 1.596125 & -1.743746 \\
\hline C & 5.188836 & 2.393234 & -0.023470 \\
\hline O & 6.017744 & 3.033489 & 0.586132 \\
\hline C & 3.163978 & 1.664645 & 1.163183 \\
\hline $\mathrm{H}$ & 3.435349 & 2.101148 & 2.130441 \\
\hline $\mathrm{H}$ & 2.079988 & 1.525133 & 1.120670 \\
\hline $\mathrm{H}$ & 3.608956 & 0.666212 & 1.114164 \\
\hline $\mathrm{H}$ & 5.977671 & 0.412377 & -0.460484 \\
\hline H & 3.687304 & -0.019998 & -1.096875 \\
\hline C & 3.177800 & 4.021238 & 0.184861 \\
\hline C & 1.636596 & 4.076379 & 0.036403 \\
\hline $\mathrm{H}$ & 3.653790 & 4.645606 & -0.581473 \\
\hline $\mathrm{H}$ & 3.473865 & 4.437062 & 1.153654 \\
\hline $\mathrm{H}$ & 1.344440 & 5.070677 & -0.327790 \\
\hline $\mathrm{H}$ & 1.181324 & 3.988142 & 1.032050 \\
\hline $\mathrm{H}$ & 1.732933 & 0.148812 & -2.551993 \\
\hline $\mathrm{H}$ & -0.718013 & -0.108789 & -2.458055 \\
\hline $\mathrm{H}$ & 3.617767 & 2.864746 & -2.090646 \\
\hline $\mathrm{H}$ & -3.801694 & 0.157452 & 2.302312 \\
\hline $\mathrm{H}$ & -2.689164 & -2.891923 & -3.199008 \\
\hline F & 2.182398 & -1.088817 & 4.163224 \\
\hline & 3.537915 & -2.992630 & -1.778980 \\
\hline
\end{tabular}

